\title{
Multi-method Modeling Framework for Support of Integrated Water Resources Management
}

Vladimir V. Nikolic, The University of Western Ontario

Supervisor: Prof. Slobodan P. Simonovic, The University of Western Ontario

A thesis submitted in partial fulfillment of the requirements for the Doctor of Philosophy degree in Civil and Environmental Engineering

(C) Vladimir V. Nikolic 2015

Follow this and additional works at: https://ir.lib.uwo.ca/etd

Part of the Other Civil and Environmental Engineering Commons

\section{Recommended Citation}

Nikolic, Vladimir V., "Multi-method Modeling Framework for Support of Integrated Water Resources Management" (2015). Electronic Thesis and Dissertation Repository. 2790.

https://ir.lib.uwo.ca/etd/2790

This Dissertation/Thesis is brought to you for free and open access by Scholarship@Western. It has been accepted for inclusion in Electronic Thesis and Dissertation Repository by an authorized administrator of Scholarship@Western. For more information, please contact wlswadmin@uwo.ca. 


\title{
Multi-method Modeling Framework for Support of Integrated Water Resources Management
}

(Thesis format: Monograph)

\author{
by \\ Vladimir Nikolic \\ Graduate Program \\ in \\ Civil and Environmental Engineering
}

\begin{abstract}
A thesis submitted in partial fulfillment of the requirements for the degree of

Doctor of Philosophy
\end{abstract}

The School of Graduate and Postdoctoral Studies

The University of Western Ontario

London, Ontario, Canada

(C) Vladimir Nikolic 2015 


\section{Abstract}

The existing definition of integrated water resources management (IWRM) promotes a holistic approach to water resources management practice. The IWRM deals with planning, design and operation of complex systems in order to control the quantity, quality, temporal and spatial distribution of water with the main objective of meeting human and ecological needs and providing protection from water disasters. One of the main challenges of IWRM is development of tools for operational implementation of the concept and dynamic coupling of physical and socio-economic components of water resources systems. This research examines the role of simulation in IWRM practices, analyses the advantages and limitations of existing modeling methods, and, as a result, suggests a new generic multi-method modeling framework that has the main goal to capture all structural complexities and interactions within water resources systems. Since traditional modeling methods solely do not provide sufficient support, this framework uses multi-method simulation approach to examine the co-dependence between natural resources and socio-economic environment. Designed framework consists of (i) a spatial database, (ii) a process-based model for representing the physical environment and changing conditions, and (iii) an agent-based model for representing spatially explicit socio-economic environment. The main idea behind multi-agent models is to build virtual complex systems composed of autonomous entities, which operate on local knowledge, possess limited abilities, affect and are affected by local environment, and thus enact the desired global system behavior. Based on the architecture of the generic multi-method modeling framework, an operational model is developed for the Upper Thames River basin, Southwestern Ontario, Canada. Six different experiments combine three climate and two socio-economic scenarios to analyze spatial dynamics of a complex physical-social-economic system. Obtained results present strong dependence between changes in hydrologic regime, in this case surface runoff and groundwater recharge rates, and regional socio-economic activities.

\section{Keywords}

Integrated Water Resources Management, Water Resources Systems, Multi-method Modeling, Agent-based modeling, Netlogo 


\section{Acknowledgments}

I would like to express my deepest gratitude to my supervisor Professor Slobodan P. Simonovic for believing in me and giving me the opportunity to study and work at the University of Western Ontario. Even when I was greatly discouraged, his enthusiasm and patience guided me throughout this challenging educational and professional life experience. Thank you, Professor Simonovic.

Also, I would like to express my sincere thankfulness to all members Western University for the effort they have invested over the years to create the stimulating environment for learning and education.

A very special appreciation I owe to my friend, Dr. Dragan Milicevic, for numerous programming advices. He and his family have always provided me with unselfish support.

Some of the knowledge I gained during the last four years will eventually be outdated and replaced, but the memories of happiness and solicitude which I shared with my FIDS colleagues - will not. They will stay with me for life. Thank you, Angela, Amin, Abhishek, Sohom, Andre, Roshan, Sarah, Patrick, Dejan and Benyou.

Despite the fact that we have been thousands of miles apart, my family has done the thing that I needed the most - they have given me such a strong support that I have never felt really alone. 


\section{Table of Contents}

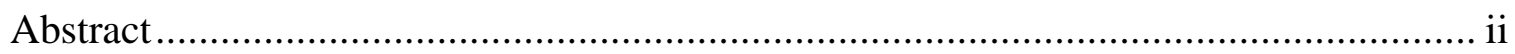

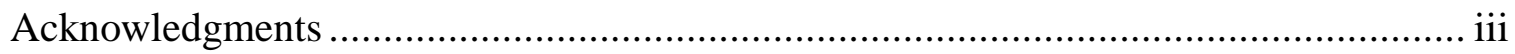

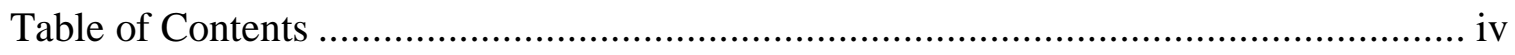

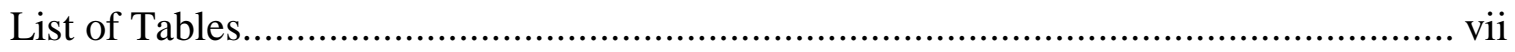

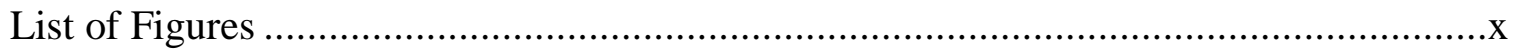

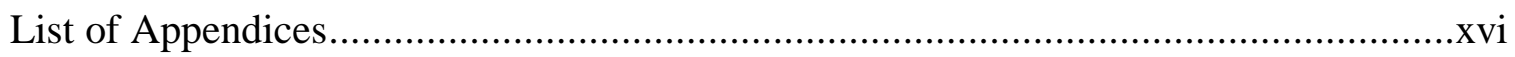

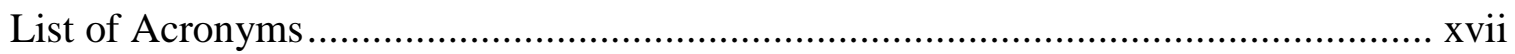

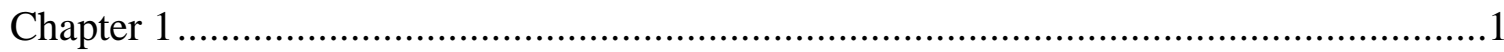

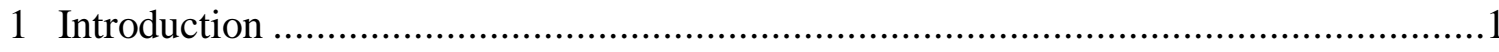

2 Modeling the complexities of water resources systems ....................................4

2.1 Water as a resource ..................................................................................4

2.2 Water resources and human activities.....................................................6

2.3 Water resources and climate change ..................................................

2.3.1 Water resources management ............................................... 10

2.4 Evolution of Integrated Water Resources Management (IWRM) ......................11

2.5 Integrated Water Resources Management (IWRM) in Canada...........................19

2.5.1 Integrated Water Resources Management (IWRM) on the Canadian

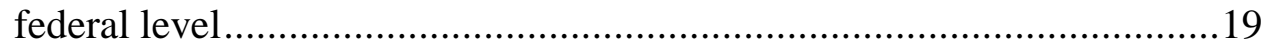

2.5.2 Integrated Water Resources Management (IWRM) in Ontario ................22

2.6 Systems approach to water resources management ....................................25

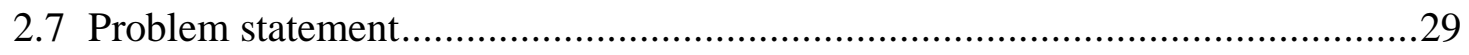

2.7.1 System Dynamics (SD) modeling method.......................................31

2.7.2 Limitations of the System Dynamics (SD) modeling method ..................33

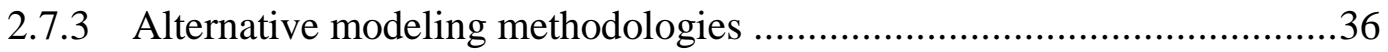


2.8 Research contributions .44

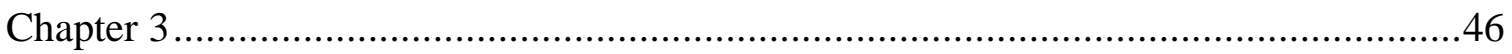

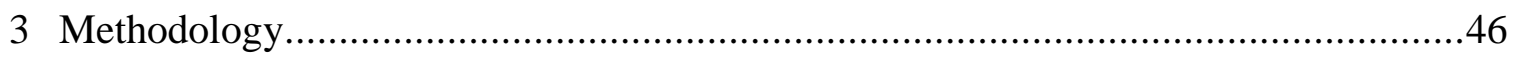

3.1 Level 1: Architecture of a generic multi-method modeling framework ..............46

3.2 Level 2: Detailed architecture of an operational model for support of integrated

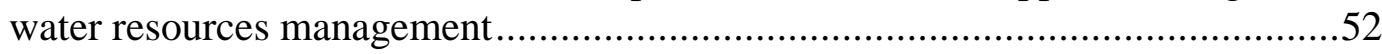

3.3 Level 3: Detailed description of individual components of an operational model for support of integrated water resources management .................................54

3.3.1 Component 1: Spatial database ......................................................54

3.3.2 Component 2: Process-based semi-distributed hydrologic model ............57

3.3.3 Component 3: Spatially explicit socio-economic agent-based model.......69

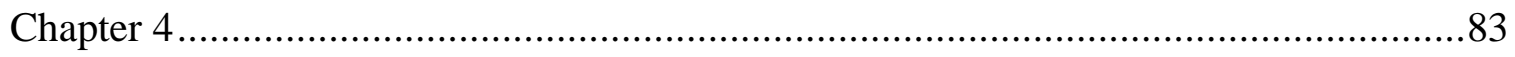

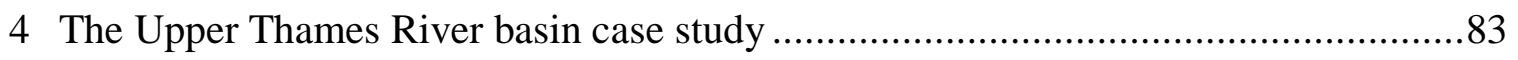

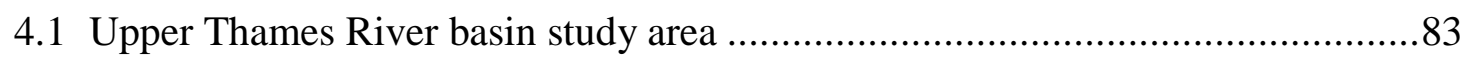

4.1.1 Physical conditions in the Upper Thames River basin .........................84

4.1.2 Water allocation in the Upper Thames River basin ...........................91

4.2 Components of the Integrated Hydrologic-Socio-Economic Model for the Upper Thames River basin (IHSEM-UTRB) .....................................................95

4.2.1 Component 1: Spatial database ..................................................95

4.2.2 Component 2: Spatially semi-distributed continuous hydrologic model 101

4.2.3 Component 3: Spatially explicit socio-economic agent-based model.....105

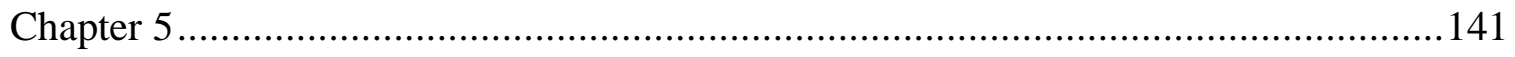

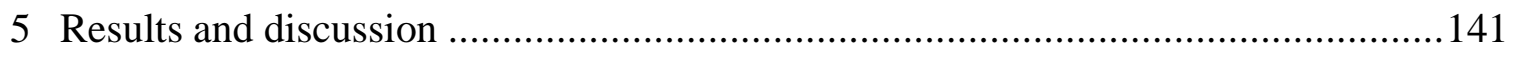

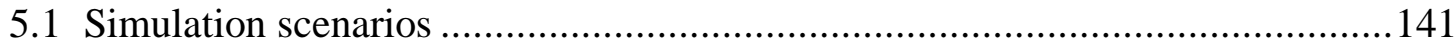

5.2 Experiment 1: the baseline socio-economic scenario (i) and historic climate conditions (a)

5.3 Experiment 2: the baseline socio-economic scenario (i) and wet climate conditions (b) 
5.4 Experiment 3: the baseline socio-economic scenario (i) and dry climate conditions (c)

5.5 Experiment 4: the infinite natural resources socio-economic scenario (ii) and

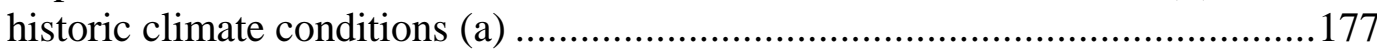

5.6 Experiment 5: the infinite natural resources socio-economic scenario (ii) and wet climate conditions (b).

5.7 Experiment 6: the infinite natural resources socio-economic scenario (ii) and dry climate conditions (c)..... 180

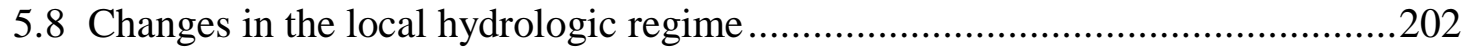

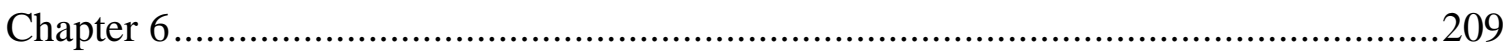

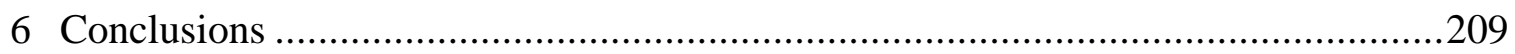

6.1 Multi-method modeling framework for support of IWRM...............................209

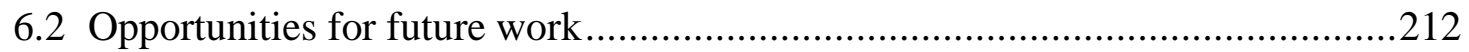

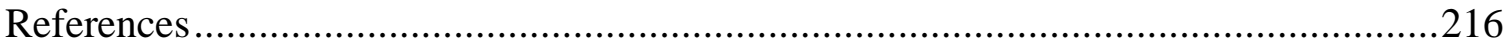

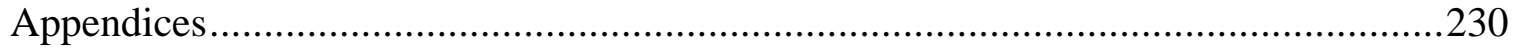

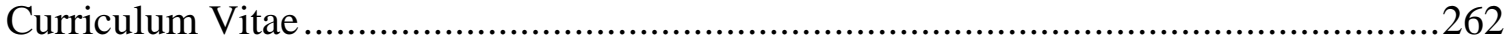




\section{List of Tables}

Table 1: Seven challenges identified by The Hague Ministerial Declaration ..................... 15

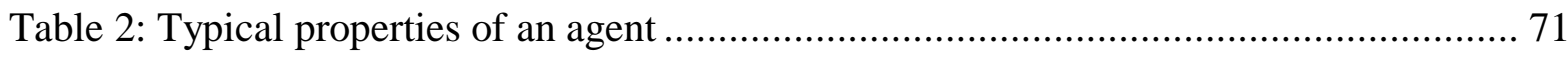

Table 3: Elements of the ODD Protocol ................................................................ 80

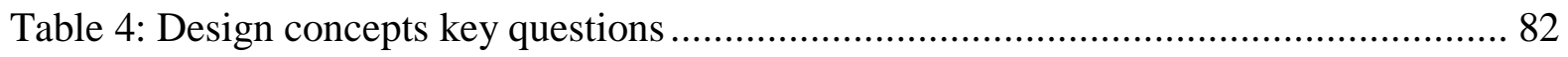

Table 5: Thames Region annual Precipitation, 1950 - 2005 in $\mathrm{mm}$............................. 85

Table 6: Land use in the Upper Thames River basin ............................................ 91

Table 7: Number of water permits by sector in Thames River basin .............................. 93

Table 8: Land-use categories in the Upper Thames River basin and their identification

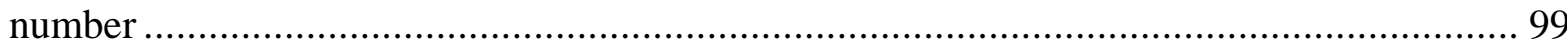

Table 9: List of 28 sub-basins in the Upper Thames River basin and their identification

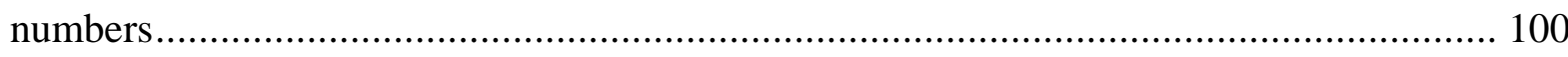

Table 10: Three counties in the Upper Thames River basin and their identification numbers 101

Table 11: Declared variables for the urban center entities ....................................... 115

Table 12: Declared variables for the industrial water users........................................ 116

Table 13: Initialization commands executed by the Model Setup button ........................ 119

Table 14: Model switches and their description ................................................. 120

Table 15: List of model sliders that are used to define the model parameters.................. 121

Table 16: List of model choosers and their description....................................... 123

Table 17: Seasonal variation of agricultural water demand ..................................... 129 
Table 18: Different types of products and their water footprint

Table 19: Seasonal water demand variation list for industrial users

Table 20: Canadian industrial water footprint $\left(\mathrm{m}^{3} / 1000 \$\right)$

Table 21: Seasonal water demand variation list for commercial water users 133

Table 22: Seasonal water demand variation list for municipal water users

Table 23: Primary water supply systems variation list

Table 24: Unpermitted and dewatering water use per sub-basin

Table 25: Seasonal variation of unpermitted water use 138

Table 26: Monthly precipitation and temperature change fields 145

Table 27: Generated meteorological data 146

Table 28: Model parameters for the baseline socio-economic scenario 148

Table 29: Specific model parameters used to describe infinite natural resources socioeconomic scenario 150

Table 30: Six combinations of climate and socio-economic scenarios 151

Table 31: Median and average monthly flows at Byron station $\left[\mathrm{m}^{3} / \mathrm{s}\right]$ 205

Table 32: Changes in total runoff volumes calculated for 20 years at Byron station $\left[\mathrm{m}^{3}\right] \ldots 205$

Table 33: Median and average monthly flows at Ingersoll station $\left[\mathrm{m}^{3} / \mathrm{s}\right]$ 206

Table 34: Changes in total runoff volumes calculated for 20 years at Ingersoll station $\left[\mathrm{m}^{3}\right] 206$ Table 35: Median and average monthly flows at St.Marys station $\left[\mathrm{m}^{3} / \mathrm{s}\right]$. 207

Table 36: Changes in total runoff volumes calculated for 20 years at St.Marys station $\left[\mathrm{m}^{3}\right]$ 
Table 37: Total groundwater recharge volumes for Middle Thames sub-basin $\left[\mathrm{m}^{3}\right]$

Table 38: Total groundwater recharge volumes for North Mitchell sub-basin $\left[\mathrm{m}^{3}\right] \ldots \ldots \ldots \ldots . .208$

Table 39: Total groundwater recharge volumes for River Band sub-basin $\left[\mathrm{m}^{3}\right] \ldots \ldots \ldots \ldots . . . .208$ 


\section{List of Figures}

Figure 1: Main elements of system dynamics simulation ......................................... 32

Figure 2: Modeling stage (2) in water resources management process............................. 48

Figure 3: Architecture of the generic multi-method modeling framework ....................... 51

Figure 4: Detailed architecture of an operational model to support integrated water resources

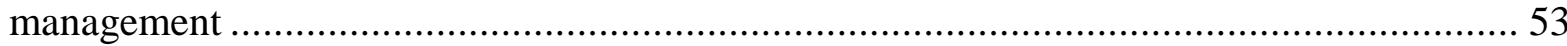

Figure 5: Modules of a hydrologic model ........................................................ 61

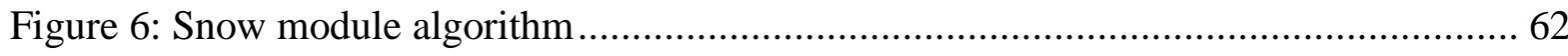

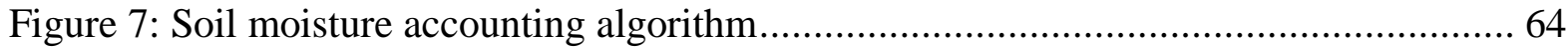

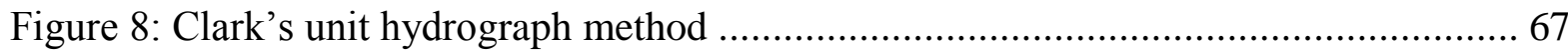

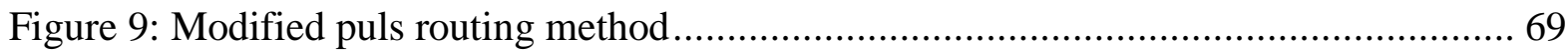

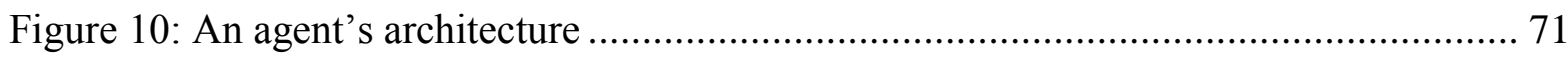

Figure 11: Typical structure of a system of agents .............................................. 72

Figure 12: Upper Thames River basin and 28 sub-basins ....................................... 85

Figure 13: Wildwood reservoir operation guidelines ......................................... 87

Figure 14: Pittock reservoir operation guidelines ........................................... 88

Figure 15: Cross-section of the Fanshawe dam ...................................................... 89

Figure 16: Upper Thames RB Hydrologic model schematics ..................................... 102

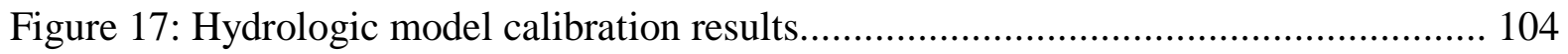

Figure 18: Main window of the model showing land-use map and active water users in each

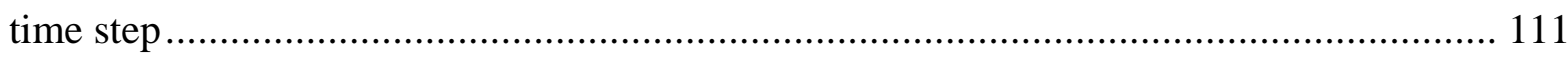


Figure 19: Recognized system actors by the socio-economic model 112

Figure 20: List of variables of an agent representing an urban center 115

Figure 21: List of variables of an agent representing an industrial water user 117

Figure 22: Model Setup button for initialization. 119

Figure 23: Model switches 120

Figure 24: Model sliders used to parameterize the model. 121

Figure 25: Model choosers 123

Figure 26: Sub-models and their order of execution 124

Figure 27: Maximum Surface Storage Effect look-up table. 126

Figure 28: Maximum Soil Infiltration Effect look-up table. 127

Figure 29: Potential Evapotranspiration look-up table 128

Figure 30: Urbanization of the City of London 136

Figure 31: Flood frequency analysis under different climate conditions, Byron station ..... 142

Figure 32: Flood frequency analysis under different climate conditions, St.Marys station . 142

Figure 33: Low flow frequency analysis under different climate conditions, Byron station 143

Figure 34: Low flow frequency analysis under different climate conditions, St. Marys station 143

Figure 35: Dynamics of land use change and active water users in the Upper Thames River basin at time step 1 (January, 2000) 157

Figure 36: Dynamics of land use change and active water users in the Upper Thames River basin at time step 61 (January, 2005) 158 
Figure 37: Dynamics of land use change and active water users in the Upper Thames River basin at time step 121(January, 2010)

Figure 38: Dynamics of land use change and active water users in the Upper Thames River basin at time step 181 (January, 2015) ..... 160

Figure 39: Dynamics of land use change and active water users in the Upper Thames River basin at time step 241 (January, 2020) 161

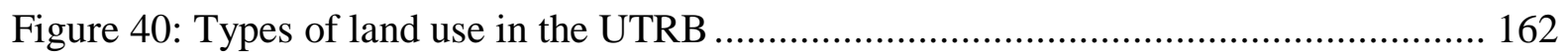

Figure 41: Population dynamics for the City of London ......................................... 162

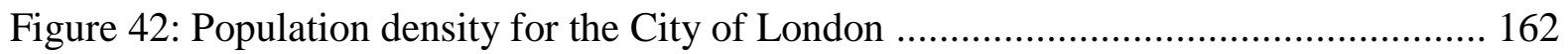

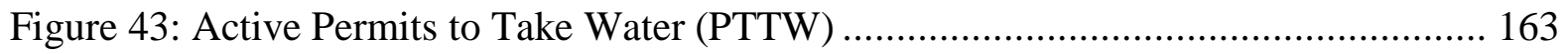

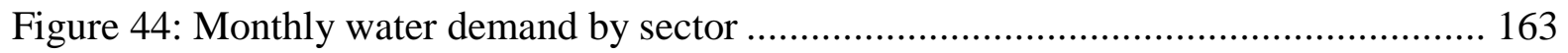

Figure 45: Industrial economic revenue based on the individual water demand ............... 164

Figure 46: Agricultural economic revenue based on the individual water demand............ 164

Figure 47: Agricultural economic revenue based on the farmed area............................ 164

Figure 48: River Thames median monthly flow rates at Byron for three climate scenarios (a,

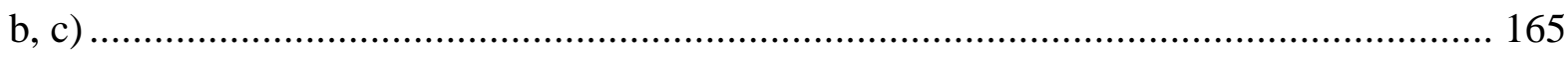

Figure 49: River Thames median monthly flow rates at Ingersoll for three climate scenarios $(\mathrm{a}, \mathrm{b}, \mathrm{c})$ 166

Figure 50: River Thames median monthly flow rates at St. Marys for three climate scenarios $(\mathrm{a}, \mathrm{b}, \mathrm{c})$ 167

Figure 51: Middlesex County groundwater recharge rates and total water demand for three climate scenarios $(a, b, c)$. 168 
Figure 52: Oxford County groundwater recharge rates and total water demand for three climate scenarios $(\mathrm{a}, \mathrm{b}, \mathrm{c})$.

Figure 53: Perth County groundwater recharge rates and total water demand for three climate scenarios $(a, b, c)$ 170

Figure 54: Middle Thames (sub-basin 14) groundwater recharge rates and total water demand for three climate scenarios $(a, b, c)$ 171

Figure 55: Middle Thames (sub-basin 14) cumulative water balance for three climate scenarios $(\mathrm{a}, \mathrm{b}, \mathrm{c})$ 172

Figure 56: North Mitchell (sub-basin 20) groundwater recharge rates and total water demand for three climate scenarios $(a, b, c)$. 173

Figure 57: North Mitchell (sub-basin 20) cumulative water balance for three climate scenarios $(\mathrm{a}, \mathrm{b}, \mathrm{c})$ 174

Figure 58: River Bend (sub-basin 27) groundwater recharge rates and total water demand for three climate scenarios $(a, b, c)$ 175

Figure 59: River Bend (sub-basin 27) cumulative water balance for three climate scenarios $(\mathrm{a}, \mathrm{b}, \mathrm{c})$ 176

Figure 60: Dynamics of land use change and active water users in the Upper Thames River basin at time step 1 (January, 2000) 182

Figure 61: Dynamics of land use change and active water users in the Upper Thames River basin at time step 61 (January, 2005) 183

Figure 62: Dynamics of land use change and active water users in the Upper Thames River basin at time step 121 (January, 2010) 184

Figure 63: Dynamics of land use change and active water users in the Upper Thames River basin at time step 181 (January, 2015) 185 
Figure 64: Dynamics of land use change and active water users in the Upper Thames River basin at time step 241 (January, 2020) 186

Figure 65: Types of land use categories in the UTRB 187

Figure 66: Population dynamics for the City of London 187

Figure 67: Population density for the City of London 187

Figure 68: Active Permits to Take Water (PTTW) 188

Figure 69: Monthly water demand by sector 188

Figure 70: Industrial economic revenue based on the individual water demand 189

Figure 71: Economic revenues for agricultural water users based on the PTTW database . 189

Figure 72: Agricultural economic revenue based on the farmed area. 189

Figure 73: River Thames median monthly flow rates at Byron for three climate scenarios (a, $\mathrm{b}, \mathrm{c})$ 190

Figure 74: River Thames median monthly flow rates at Ingersoll for three climate scenarios $(\mathrm{a}, \mathrm{b}, \mathrm{c})$ 191

Figure 75: River Thames median monthly flow rates at St. Marys for three climate scenarios $(\mathrm{a}, \mathrm{b}, \mathrm{c})$ 192

Figure 76: Middlesex County groundwater recharge rates and total water demand for three climate scenarios $(\mathrm{a}, \mathrm{b}, \mathrm{c})$ 193

Figure 77: Oxford County groundwater recharge rates and total water demand for three climate scenarios $(\mathrm{a}, \mathrm{b}, \mathrm{c})$ 194

Figure 78: Perth County groundwater recharge rates and total water demand for three climate scenarios $(a, b, c)$ 195 
Figure 79: Middle Thames (sub-basin 14) groundwater recharge rates and total water demand

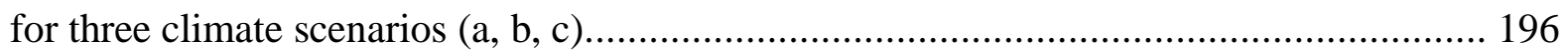

Figure 80: Middle Thames (sub-basin14) cumulative water balance for three climate scenarios $(\mathrm{a}, \mathrm{b}, \mathrm{c})$

Figure 81: North Mitchell (sub-basin 20) groundwater recharge rates and total water demand for three climate scenarios $(a, b, c)$ 198

Figure 82: North Mitchell (sub-basin 20) cumulative water balance for three climate scenarios $(\mathrm{a}, \mathrm{b}, \mathrm{c})$ 199

Figure 83: River Bend (sub-basin 27) groundwater recharge rates and total water demand for three climate scenarios $(\mathrm{a}, \mathrm{b}, \mathrm{c})$ 200

Figure 84: River Bend (sub-basin 27) cumulative water balance for three climate scenarios $(\mathrm{a}, \mathrm{b}, \mathrm{c})$

Figure 85: Monthly distribution of precipitation for three climate scenarios for London Airport CS 


\section{List of Appendices}

Appendix A Spatial database - list of attributes

Appendix B Creating the hydrologic extension in Netlogo

Appendix C Netlogo variables

Appendix D IHSEM-UTRB Model installation 


\title{
List of Acronyms
}

\author{
ABM Agent-based Modeling \\ ASCII American Standard Code for Information Interchange, \\ CA Cellular Automata \\ CA Conservation Authority \\ CSSD Compartmental Spatial System Dynamics \\ DBMS Database Management System \\ DDE Dynamic Data Exchange \\ DEM Digital Elevation Model \\ GCM Global Circulation Model \\ GDP Gross Domestic Product \\ GIS Geographic Information System \\ GWP Global Water Partnership \\ HEC-HMS Hydrologic Engineering Center - The Hydrologic Modeling System \\ IHSEM-UTRB Integrated Hydrologic-Socio-Economic Model for the Upper Thames River basin \\ IPCC Intergovernmental Panel on Climate Change \\ IWRM Integrated Water Resources Management \\ MSI Maximum Surface Infiltration Capacity \\ MSS Maximum Surface Storage \\ NEA National Ecosystem Assessment \\ ODD Overview, Design Concepts, and Details \\ PET Potential Evapotranspiration \\ PTTW Permit to Take Water \\ PWSS Primary Water Supply System \\ UTRB Upper Thames River Basin \\ UTRCA Upper Thames River Conservation Authority




$\begin{array}{cl}\text { SD } & \text { System Dynamics } \\ \text { SIDA } & \text { Swedish International Development Agency } \\ \text { SSD } & \text { Spatial System Dynamics } \\ \text { SQL } & \text { Structured Query Language } \\ \text { UN } & \text { United Nations } \\ \text { UNDP } & \text { United Nations Development Program } \\ \text { WRM } & \text { Water Resources Management } \\ \text { WRS } & \text { Water resources System } \\ \text { WWAP } & \text { World Water Assessment Programme }\end{array}$




\section{Chapter 1}

\section{Introduction}

A number of global natural and socio-economic processes, such as climate change, rapid population growth, and substantial land use change are placing significant pressures on water resources. All these pressures extensively disturb human access to water of good quality and quantity, and, therefore, directly influence social, economic and physical well-being of both people and natural ecosystems. The research presented here adopts the Integrated Water Resources Management (IWRM) approach that recognizes that the social, economic and environmental processes are an inseparable part of the management of natural resources. This approach emphasizes the need for coordination in the development and management of water, land and other related resources. The main objective of the IWRM is to maximize economic and social welfare in an equitable manner without compromising ecosystem sustainability. The integrated water resources management deals with planning, design and operation of complex water resources systems in order to control the quantity, quality, temporal and spatial distribution of water with the main objective of meeting human and ecological needs and providing protection from water related hazards. The complexity of water resources systems originates from the interactions of three main sub-systems:

- The natural river system in which physical, chemical, and biological processes take place;

- The socio-economic system which includes all human activities related to the use of the natural river and land systems;

- The administrative and institutional system of administration, legislation and regulation, where the decision, planning and management processes take place.

Computer simulations play a central role in all aspects of management of water systems because they provide substantial information to support informed decision-making. From 
the perspective of the water community, a simulation model represents a simplified but purposeful representation of a real-world water resources system.

One of the main challenges of IWRM is development of tools for operational implementation of the concept and dynamic coupling of physical and socio-economic components of water resources systems. Literature suggests that a number of models have been developed with the main idea of finding the optimal management strategies. However, only a small number of models interactively analyze how physical aspects of water resources systems affect and are affected by the social, economic and environmental sub-systems. The majority of developed models ignores the interaction between system components, the non-linearity of a system, the feedback mechanisms, and, most importantly, ignores the explicit representation of spatial characteristics of water resources systems.

System dynamics simulation is one of the modeling methods that is capable of coupling both the physical and socio-economic processes. System dynamics is able to capture the feedback structure of different system components within a single modeling framework, and represents a very useful tool in integrated water resources management. However, system dynamics modeling method comes with one important limitation regarding the presentation of water resources systems - it does not support the explicit representation of spatial system elements and spatial variability within a modeled system.

This research focuses on the role of simulation in integrated water resources management process, and analyses the specific advantages and limitations of existing modeling methods from the aspect of spatial representation of water resources systems. The main objective is to find the optimal combination of different modeling methods, and to define the architecture of a multi-method modelling framework which is able to represent all the structural complexities and interactions within a water resources system. The proposed research adopts the multi-method simulation approach to address the interconnectedness and important feedbacks that are characteristic for water resources systems. Emphasis is placed on explicit modeling and simulation of the key aspects of the complexity of water resources systems, including: 
1. Feedback based system structure;

2. Integral representation of physiographic, environmental and socio-economic sub-systems, and their non-linear interactions;

3. Explicit representation of complex spatial and temporal characteristics of water resources systems;

The main contributions of this research are:

1. Definition of a generic modeling framework that captures the feedback processes in time and space to describe the interaction between natural resources and social and economic environment. Designed modeling framework methodology must be designed to support the main principles of integrated water resources management in such a way that it can be applied to other physical, social, economic and environmental contexts and potential problems related to management of water resources;

2. Development of a user-friendly operational model to support the integrated water resources management in the Upper Thames River Basin, Ontario, Canada, based on open source computational platforms.

In addition to Chapter 1, this thesis contains five more chapters. Chapter 2 details the evolution and contemporary definition of integrated water resources management and analyzes the main problems of modeling of water resources systems. As a result, this chapter clearly defines the main research problem and lists the expected research contributions. The third chapter explains the new multi-method modeling framework that is developed according to the requirements of IWRM and representation of water resources systems. Chapter 4 demonstrates the practical implementation of newly suggested methodology on the Upper Thames River basin case study, while Chapter 5 discusses the obtained results. The final conclusions and suggestions for the future research are given in Chapter 6. 


\section{Chapter 2}

\section{Modeling the complexities of water resources systems 2.1 Water as a resource}

Water is unique among physical elements in the role it plays in nature. Life indeed consists mostly of water. This is as true of amoebae as it is of man. Water holds together the web of life. Ever since the first words were spoken on earth, water has shaped social, economic, cultural and environmental landscapes. Ancient societies thrived along bodies of water, used for sustenance as well as transport. With increased human activity and the development of technology in recent times, water as a resource finds itself under tremendous pressure. United Nations warn that the world's fundamental natural resources (water, food, and energy) are exposed to significant stresses and predict a substantial increase in pressures in the near future (WWAP, 2012). Growing pressure is primarily placed in the context of climate change, rapid growth of the human population, urbanization, and resulting increases in consumption.

Traditionally, natural resources were considered an infinite and inexhaustible gift of nature. With the boom in population and consumption, humanity is slowly beginning to realize the limits of this gift. While $70 \%$ of our planet is covered with water - hence the Blue Planet - the concern is the available fresh water, and its spatial and temporal distribution over different regions of the world (Constance, 2004). Only $2.5 \%$ of the total volume of water is fresh water, the remainder is saline. Of the $2.5 \%$, approximately $70 \%$ is captured in the ice caps of Antarctica and Greenland. Part of the remainder exists as soil moisture, or lies in deep underground aquifers not easily reachable for human use, which leaves less than one percent directly accessible for utilization. This water is to be found in rivers, reservoirs, lakes and higher groundwater aquifers.

The spatial distribution of the planet's fresh water resources and their rates of renewal can differ extremely due to the complexity of the underlying global hydrologic cycle. The global hydrologic cycle is a result of an energy and water exchange between the atmosphere, the land, and the oceans. The components of the hydrologic cycle are 
precipitation over land and ocean on the one hand, and runoff and evaporation from them on the other. Globally, the cycle brings an average annual precipitation of about $990 \mathrm{~mm}$, while the average annual rainfall over the continents is about $746 \mathrm{~mm}$ (Constance, 2004). This fact does not address significant variations between different regions in amounts of seasonal and annual precipitation. Some humid regions in tropical areas have frequent and intensive precipitations with the total annual amount higher than $11.000 \mathrm{~mm}$, while, at the same time, certain regions, such as the South American Atacama Desert, are left practically dry with negligible annual precipitation (WWAP, 2012).

Additional concerns arise from the fact that there is an intensifying disproportion between the concentration of the human population and the distribution of available fresh water resources. In order to analyze the spatial and temporal variability of available water resources with respect to the concentration of human population across different world regions, Shiklomanov (2000) defines and utilizes the specific water availability index. The index represents the value of actual per capita renewable water resources, and it is calculated by dividing the quantities of available water resources without water consumption by the population number. The specific water availability then represents the residual per capita quantity of fresh water. Studies prepared in 1950s showed that the major portion of our planet had the specific water availability index around or above the average, with the exception of Northern African regions. At the same time, this index was low in Central and South Europe, North China, and South Asia. Due to variations in the hydrologic cycle and continuous global population growth, by 1995, the situation was drastically different. Municipal water supply sharply decreased in many regions, becoming very low in some (e.g. Northern China, Southern and Western Asia), and catastrophically low in others (e.g. North Africa and Arabian Peninsula). By 2025, it is estimated that approximately $30-35 \%$ of the world's population will have low or catastrophically low water supply, that is less than $1,000 \mathrm{~m}^{3}$ per year per capita (Shiklomanov, 2000). At the same time, some regions are expected to have higher water availability, such as Northern Europe, Canada, Alaska, South America, Central Africa, Siberia, and Oceania. Shiklomanov (2000) in his studies divides the world into three regions: industrially developed countries, developing countries with sufficient or extensive moisture, and developing countries in arid and semiarid regions. He recognizes 
that an extensive natural unevenness of water availability over the earth increases over time by significant rates primarily as a result of human socio-economic activities, rapid population growth and variability in climate conditions.

\subsection{Water resources and human activities}

Since the dawn of human history, water resources played a key part in most aspects of human life. The role of water in life remains irreplaceable. As humanity grew and developed, it stayed inseparably weaved into the ecosystem and the underlying global water cycle. Nowadays, water is an essential resource for household operation, agricultural practices, industrial manufacturing, energy production, navigation, and recreation.

Most importantly, safe access to clean water supplies and a basic sanitation infrastructure are essential for preserving public health. It is anticipated that the world's urban population will grow from 3.4 billion in 2009 to 6.3 billion in 2050 (Cosgrove and Cosgrove, 2011). It can be assumed that problems of adequate water supply, sanitation, and drainage will shadow this trend, making large megacities of developing countries especially vulnerable. Current estimates show that 1.2 billion people have no sanitation facilities whatsoever, while 2.5 billion people lack access to developed sanitation (WHO, 2014).

The quality of water supply is also very important. For healthy socio-economic development, it is important that the available water meet the safety standards. Poor water quality has direct economic consequences, including degradation of economic services, costs related to endangered human health, impacts on economic activities such as agriculture, industrial production and tourism. Addressing all this means increased costs of water treatment. Approximately 2 million tons of waste per year is deposited into bodies of water, including industrial waste, chemicals, human and agricultural waste (pesticides, pesticide residues and fertilizers). In developing countries, the share of sewage discharge without prior treatment is above $80 \%$, and this waste directly pollutes rivers, lakes, and coastal areas, making groundwater aquifers especially vulnerable as they are extremely costly and difficult to clean (WHO, 2014). Estimates show that, at any 
one time, more than half of the underprivileged population in the developing world suffers from water-borne diseases due to lack of proper hygiene, sanitation and adequate water supply.

In agriculture, large amounts of water are required for irrigation and food production practices. Today, water use for irrigation and food production constitutes the single greatest pressure on freshwater resources. World Water Assessment Programme (WWAP) of the United Nations estimates that the global groundwater withdrawal has tripled over the last 50 years, and suggests that this fact has "fundamentally changed the role of groundwater in human society and irrigation sector" (WWAP, 2012). 70\% of global freshwater abstractions go to agriculture, roughly 3100 billion $\mathrm{m}^{3}$ (FAO, 2010). In some fast-growing economies, the figure is closer to $90 \%$. Projected global population growth of 2 to 3 billion people over the next 40 years is predicted to increase the food demand by $70 \%$ by 2050 . This will likely bring water use to 4500 billion $\mathrm{m}^{3}$ by 2030 .

Global and regional energy sectors also depend strongly on water resources. Hydropower creates $\sim 20 \%$ of the world's electricity. It is a key source of renewable energy worldwide. Water is required for powering turbines, cooling thermal power plants, and growing biofuels. While over a billion of people still lack access to electricity (WWAP, 2012), the anticipated demographic growth and improvements to the standard of living worldwide are expected to create a surge in energy consumption. While the water used in hydropower generation returns to the source (the river) after passing through the turbines, substantial losses do occur, however, through evaporation from reservoirs, and extensive quantities of water will be needed to accommodate increasing demands in the future.

\subsection{Water resources and climate change}

Global and local natural resources fundamentally depend on the climate conditions. The climate is one of the most complex natural systems and can be defined as the full range of weather conditions experienced in a particular place over long period of time, including daily, seasonal and annual variations. Many factors determine the climate of a particular region, and are usually divided into two categories: primary and secondary factors. Primary factors include latitude, elevation, topography of the terrain and the amount of 
solar radiation. Secondary factors involve ocean currents, wind systems and other natural cycles.

The Intergovernmental Panel on Climate Change (IPCC), established in 1988 under the jurisdiction of the United Nations, defines climate change as "a change in the state of the climate that can be identified by changes in the mean and/or the variability of its properties, and that persists for an extended period, typically decades or longer", (IPCC, 2013). Shifts in climate regimes are driven by variations in complex natural processes (for example, solar radiation and natural phenomena such as volcanic activities), and by a number of human-induced forces, such as emissions of greenhouse gasses into the atmosphere, or large-scale changes in land use patterns. All these processes disrupt the energy balance of the climate system, and, therefore, change the global temperature and precipitation patterns. Small variations in solar radiation and volcanic activities alter significantly the energy balance, often resulting in large temperature changes. In addition, the greenhouse gasses confined in atmosphere prevent excess heat to escape into space; while large-scale changes in land use reduce Earth's potential to capture carbon dioxide from the atmosphere. Both of these processes contribute to global warming patterns.

From the hydrologic perspective, the alternating climate brings changes in temperature, rates of evapotranspiration and precipitation. This inevitably influences the rates of groundwater recharge, as well as the spatial and temporal distribution of river flows. According to IPCC, the most important traits of climate change are the altered frequencies and intensities of extreme weather conditions, (IPCC, 2013). It is supposed that climate variations bring major shifts in timing and magnitude of hydro-climatic extremes. In addition to the rise of sea level, it is anticipated that these alterations will have adverse effects on natural and human systems on a global scale, with floods, droughts, typhoons and cyclones increasing in frequency. It is difficult to precisely quantify the future impact of climate change on water resources, but there are clear trends that must be carefully studied. According to WWAP (2012), the global effects of climate change on water resources include extensive changes in the distribution of precipitation, including inter-annual precipitation variability and seasonal shifts in stream flows. Increased flooding in some regions and lower summer precipitation in others will likely 
lower the yield of some groundwater aquifers, reducing the quantities of stored water in reservoirs fed by seasonal flow. IPCC summarizes the global effects of climate change on water resources as follows (IPCC, 2013):

- Some world regions are expected to experience increase, and others decrease, in streamflow volumes;

- The rates of streamflow and groundwater recharge will significantly vary between regions, and will closely follow the changes in precipitation rates;

- High streamflow will likely move from spring to winter for rivers that depend on snowmelt, with significantly lower flows during the summer season;

- The increase in water temperatures will likely cause the water quality to deteriorate;

- Flood frequencies and magnitudes are likely to increase in most regions;

- Low flow volumes are expected to decrease;

On the global scale, one of the most evident effects of climate change is the rise of sea level at an average rate of $3.4 \mathrm{~mm} /$ year in the past 15 years. This is almost double the rate of the previous 50 years. More locally, changing climate conditions have already altered the historic temperature and precipitation patterns across Canada. Over the last several decades precipitation levels have increased significantly in almost all regions of Canada. On the federal scale, the total average precipitation has increased by $5 \%$, while a $12 \%$ increase is reported for the southern regions. As well, in the western regions, the total rainfall to snowfall ratio has been decreasing (Barrow et al., 2004).

These changes in temperature and precipitation naturally led to changes in the flow patterns of Canadian rivers. As expected, the maximum annual and mean daily flows have been increasing in Southern Ontario, Northern British Columbia and Yukon Territory, and decreasing in Southern British Columbia (Environment Canada, 2004). Analysis of historical trends in the period between 1967 and 2003 showed a decrease of 
$10 \%$ in annual river discharge for rivers located in the northern regions of Canada (Dery, 2005).

Variations in climate extremes can be very detrimental to the socio-economic environment and human wellbeing. Between 1990 and 2000, in several developing countries, natural disasters were responsible for losses of $2 \%-15 \%$ of the countries' annual GDP (World Bank, 2013). According to the United Nations Office for Disaster Risk Reduction, water-related disasters account for $90 \%$ of all natural disasters, and their frequency and intensity is generally rising. Some 373 natural disasters killed over 298.800 people in 2010, affecting some 208 million others, and costing nearly 110 million \$US (EM-DAT, 2014). In Canada, the frequency and severity of flooding has increased over the last several decades. $62 \%$ of these flooding events were caused by snowmelt runoff, storm rainfall events or the combination of both (Brooks et al., 2001). In the period between 1990 and 1997, 168 flood disasters were witnessed in densely populated areas. Majority of these events occurred in Ontario (37), New Brunswick (26), Quebec (23) and Manitoba (18). The numbers are lower for the less-densely populated Northwestern Territories (5) and Yukon (3), (Schrubsole et al., 2003).

In the Southwestern Ontario, the analysis of historical records shows a shift toward milder winters and warmer summers, while the projected mean temperature and precipitation values are expected to increase in the future. Climate change is expected to bring a decreased runoff, increased winter and spring flows, lower summer and fall runoffs, and an increased frequency of high flows (Lemmen et al., 2008).

\subsubsection{Water resources management}

The unbreakable bond between water resources and the human civilization has placed considerable pressures on water resources over time. Natural variations in the hydrologic water cycle and a lack of appropriate management practices have caused 884 million people today to lack permanent access to a safe and clean water supply. This causes 3.575 million people to die each year from water borne diseases, $84 \%$ of whom are children 0-14 years old (UNEP, 2012). 
During the last several decades it has become apparent that water resources are limited indeed, and sustainable development of human society depends on managing them wisely. The pressure is rapidly mounting, and serious measures will have to be taken if disasters on a global scale are to be averted. Because the problem is so vital and multifaceted, as well as of such a large scale, a holistic approach is clearly necessary.

\subsection{Evolution of Integrated Water Resources Management (IWRM)}

In varying ways, local communities have managed water resources at all times with the sole objective of satisfying their own needs. Typically, a traditional approach to water management involved altering local environmental conditions by artificial manipulation of water (and other natural resources) through engineered structures or administrative measures, without consideration for other water users' needs (Simonovic, 2009b). Until recently, the effects of utilization of water resources were globally insignificant, and mainly considered to be a local concern. In an increasingly interdependent and interconnected contemporary world, however, the situation is very different. On the one hand, there is a rising demand for natural and water resources globally due to rapid population growth and economic development. On the other, distribution of water resources is constantly being altered on a local level due to climate change and other, mainly human factors. Because of social, economic and infrastructural interconnectedness of modern society, actions taken at different levels can have significant potential impacts on both local and global water resources, as well as other water users across different sectors. The growing complexity of pressures on water resources calls for a new approach to water resources management. It is now universally recognized that only coordinated management of water, land and related resources can address the increasingly complex problems adequately. The concept of Integrated Water Resources Management (IWRM) was thus introduced, and is now being promoted and implemented throughout the international community.

As a concept, IWRM has gone through a number of stages and evolved over time on the basis of experience of practitioners and decision makers. From the historical perspective, a number of key moments in IWRM evolution can be clearly distinguished, starting with 
the introduction of initial principles related to water resources and finishing with the contemporary definition of IWRM. First fragments of IWRM emerged several decades ago, in 1977, at the first United Nations Global Water Conference in Mar del Plata. Nearly 40 years later, the water community deems that this conference had a critical impact on the treatment of water related issues around the world. The Global Water Conference named the 1980s as the International Water Supply and Sanitation Decade, setting a vital goal: to provide reliable access to clean water and adequate sanitation to all citizens of the world by the end of the decade. Shortly after, the UN General Assembly officially announced the Decade and advised all UN members to work toward the proclaimed goals. It was not until Agenda 21 and the World Summit on Sustainable Development in Rio de Janeiro (1992) that the concept was seriously discussed from a practical standpoint. In January 1992, in preparation for the Rio Summit, nearly 500 governmental experts from over 100 countries held the International Conference on Water and the Environment in Dublin, Ireland. The key outcome of this conference was the establishment of the four Dublin Principles, which gave a strong foundation to the freshwater resources management component of the United Nation Agenda for the Protection of Freshwater Resources, also known as Agenda 21. The four Dublin Principles are:

Principle 1: Freshwater is a finite and vulnerable resource, essential for sustaining life, development and the environment.

Principle 2: Water development and management should be based on a participatory approach, involving users, planners and policy makers at all levels.

Principle 3: Women play a central part in the provision, management and safeguarding of water.

Principle 4: Water has an economic value in all its competing uses and should be recognized as an economic good.

Agenda 21 is seen as a non-binding and voluntary action plan that suggests a set of strategies to be implemented at different levels (globally, regionally, and locally) by the United Nations Organizations, Governments, and Major Groups in all areas in which 
"human activities alter and affect the environment". Agenda 21, the Rio Declaration on Environment and Development, and the Statement of Principles for the Sustainable Management of Forests were adopted on Earth Summit by more than 178 Governments at the United Nations Conference on Environment and Development (UNCED), Rio de Janeiro, Brazil, June 3 to 14, 1992. From a water resources management perspective, Chapter 18 of Agenda 21 is of particular interest. Chapter 18 was adopted on an intergovernmental level and is entitled "Protection of the Quality and Supply of Freshwater Resources: Application of Integrated Approaches to the Development, Management and Use of Water Resources". For decision makers and practitioners, Chapter 18 of Agenda 21 together with the Dublin Principles provides a simple, understandable and useful basis for water resources management and sustainable development, and has greatly influenced all future definitions of integrated water resources management (UN, 1992). Chapter 18 states that:

1. Freshwater resources are an essential component of the Earth's hydrosphere and an indispensable part of all terrestrial ecosystems. The freshwater environment is characterized by the hydrological cycle, including floods and droughts, which in some regions have become more extreme and dramatic in their consequences. Global climate change and atmospheric pollution could also have an impact on freshwater resources and their availability and, through sea-level rise, threaten low-lying coastal areas and small island ecosystems.

2. Water is needed in all aspects of life. The general objective is to make certain that adequate supplies of water of good quality are maintained for the entire population of this planet, while preserving the hydrological, biological and chemical functions of ecosystems, adapting human activities within the capacity limits of nature and combating vectors of water-related diseases. Innovative technologies, including the improvement of indigenous technologies, are needed to fully utilize limited water resources and to safeguard those resources against pollution.

3. The widespread scarcity, gradual destruction and aggravated pollution of freshwater resources in many world regions, along with the progressive encroachment of 
incompatible activities, demand integrated water resources planning and management. Such integration must cover all types of interrelated freshwater bodies, including both surface water and groundwater, and duly consider water quantity and quality aspects. The multi-sectoral nature of water resources development in the context of socio-economic development must be recognized, as well as the multi-interest utilization of water resources for water supply and sanitation, agriculture, industry, urban development, hydropower generation, inland fisheries, transportation, recreation, low and flat lands management and other activities. Rational water utilization schemes for the development of surface and underground water-supply sources and other potential sources have to be supported by concurrent water conservation and wastage minimization measures. Priority, however, must be accorded to flood prevention and control measures, as well as sedimentation control, where required.

4. Trans-boundary water resources and their use are of great importance to riparian States. In this connection, cooperation among those States may be desirable in conformity with existing agreements and/or other relevant arrangements, taking into account the interests of all riparian states concerned.

5. The following program areas are proposed for the freshwater sector:

- Integrated water resources development and management;

- Water resources assessment;

- Protection of water resources, water quality and aquatic ecosystems;

- Drinking-water supply and sanitation;

- Water and sustainable urban development;

- Water for sustainable food production and rural development;

- Impacts of climate change on water resources.

Several years later, in March 2000, the Hague Ministerial Declaration was presented by the 2nd World Water Forum. In order to achieve water security this declaration identified seven challenges for future action presented in Table 1. 
Table 1: Seven challenges identified by The Hague Ministerial Declaration

\begin{tabular}{|c|c|}
\hline Meeting basic needs: & $\begin{array}{l}\text { To recognize that access to safe and sufficient water and } \\
\text { sanitation are basic human needs and are essential to health } \\
\text { and well-being, and to empower people, especially women, } \\
\text { through a participatory process of water management. }\end{array}$ \\
\hline $\begin{array}{l}\text { Securing the food } \\
\text { supply: }\end{array}$ & $\begin{array}{l}\text { To enhance food security, particularly of the poor and } \\
\text { vulnerable, through the more efficient mobilization and use, } \\
\text { and the more equitable allocation of water for food } \\
\text { production. }\end{array}$ \\
\hline Protecting ecosystems: & $\begin{array}{l}\text { To ensure the integrity of ecosystems through sustainable } \\
\text { water resources management. }\end{array}$ \\
\hline Sharing water resources: & $\begin{array}{l}\text { To promote peaceful cooperation and develop synergies } \\
\text { between different uses of water at all levels, whenever } \\
\text { possible, within and, in the case of boundary and trans } \\
\text { boundary water resources, between states concerned, through } \\
\text { sustainable river basin management or other appropriate } \\
\text { approaches. }\end{array}$ \\
\hline Managing risks: & $\begin{array}{l}\text { To provide security from floods, droughts, pollution and } \\
\text { other water-related hazards. }\end{array}$ \\
\hline Valuing water: & $\begin{array}{l}\text { To manage water in a way that reflects its economic, social, } \\
\text { environmental and cultural values for all its uses, and to } \\
\text { move towards pricing water services to reflect the cost of } \\
\text { their provision. This approach should take account of the } \\
\text { need for equity and the basic needs of the poor and the } \\
\text { vulnerable. }\end{array}$ \\
\hline Governing water wisely: & $\begin{array}{l}\text { To ensure good governance, so that the involvement of the } \\
\text { public and the interests of all stakeholders are included in the } \\
\text { management of water resources. }\end{array}$ \\
\hline
\end{tabular}

The next step toward the definition of integrated water resources management was taken on September 8, 2000, when the General Assembly of the United Nations at the $8^{\text {th }}$ Plenary of the United Nations Millennium Declaration adopted a resolution titled "Protecting our common environment". In this declaration, the UN urges its members to put an end to "unsustainable exploitation of water resources by developing water management strategies at the regional, national and local levels, which promote both equitable access and adequate supplies".

In order to create an effective institutional mechanism responsible for water resources management, a new coordinating organization, the Global Water Partnership (GWP), was formed in 1996 by the World Bank, the United Nations Development Program (UNDP), and the Swedish International Development Agency (SIDA). GWP's principal mission is 
to actively identify critical knowledge needs at global, regional and national levels, help design programs for meeting those needs, and serve as a mechanism for alliance building and information exchange on water resources management. Based on the extensive heritage of water community, GWP identifies the following major challenges of water resources management, GWP (2000):

- Securing adequate water for human society;

- Protection of ecosystems (flora and fauna);

- Management of the variability of water and its associated risks;

- Raising public awareness and creating the political will to act;

- Ensuring collaboration across all sectors and boundaries.

Furthermore, GWP has since introduced and promoted the widely-accepted definition of integrated water resources management which combines the main ideas of the holistic approach, Dublin Principles, Chapter 18 of Agenda 21, and The Declaration of 2nd World Water Forum, (Ota, 2009):

"Integrated water resources management is a process which promotes the coordinated development and management of water, land, and its related resources, in order to maximize resultant economic and social welfare in an equitable manner without compromising the sustainability of vital ecosystem."

Compared to the traditional approaches to water management, the GWP emphasizes the shifts that the integrated approach brings:

- From sectorial to integrated management;

- From supply fixes to demand management;

- From command and control to more cooperative or distributive forms of governance;

- From closed expert driven management organizations to more open, transparent, and communicative bodies; and, finally,

- From top-down to stakeholder and demand responsive change. 
Explaining the evolution of the term "integration", Mohamed Kadi, Chair of GWP Technical Committee, suggests that it originally referred to "bringing together water resources with engineering and economic driven solutions" (Martinez-Santos et al., 2014). However, after gaining some practical experience in IWRM implementation, it was understood that the management of land has significant effects on water resources too, water quality in particular. This led to a realization that water quantity cannot be managed in isolation from water quality. Moreover, GWP recognized the need to build "bridges between human and natural systems, and between the water sector and the economy". Vertical integration of responsibilities was also needed across the different decision-making levels, from local, provincial, and national to river basin and international scales. Kadi further explains that the idea of integration includes more decentralized and, more holistic approaches that include an appreciation of local ideas.

Consequently, one of the main benefits of IWRM is a shift from the traditional "topdown" approach, which limits itself to technical and engineering aspects of a given water-resources problem. In contrast, the newly-advocated "bottom-up" approach seeks to bring together various socio-economic and institutional sectors for the purpose of building the management capacity needed for effective administration of water resources (Prodanovic and Simonovic, 2010). Experience shows that the state of local water resources considerably determines the level of centralized influence on the management of water (Hassing et al., 2009). Active involvement of water users and other interested parties is posed as essential to the success of IWRM. A bottom-up approach is gaining acceptance, as it considers technical, socio-economic, as well as administrative and institutional aspects of water management, where the institutional roles describe the sharing authority and responsibility between local levels, basin levels, and a centralized level. As an example of a successfully applied bottom-up approach one can analyze the South African case. In year 2000, local climate conditions caused massive water scarcity and a re-examination of established water rights. Water allocation programs became necessary and a compulsory licensing process was initiated based on the National Water Act of 1998. Existing water rights were cancelled, and all water users had to apply for it again if needed. Water licenses were made time-bound and the links between land ownership and water licenses were separated (Hassing et al., 2009). 
From the practical and operational standpoint, IWRM is seen as an ecosystem approach followed by a set of ideals (Mitchell et al., 2014):

- The river basin is the most appropriate operational management unit, rather than some other administrative or political spatial unit;

- Attention is directed to upstream - downstream, surface - ground water and quantity - quality interactions;

- Interconnections of water with other natural resources and the environment is considered;

- Environmental, economic and social aspects receive attention;

- Stakeholders are actively engaged in planning management and implementation to achieve an explicit vision, objectives and outcomes.

From the practical standpoint, IWRM has proved to be a holistic approach that must be adapted to different local and regional contexts. It cannot be "blindly" applied to any arbitrary social or environmental context since water problems differ significantly from one region to another. IWRM is therefore really a set of recommendations for important management aspects to be considered at different levels of organization. It guides decision makers to choose the most appropriate set of suggestions, policy measures, management tools and institutional arrangements in a given social, economic, and environmental context. As a process, IWRM has evolved over time, but the experience shows that it will take decades before IWRM becomes fully established in the world of rapidly increasing scarcity and competition for water. In preparation for the Rio+20 Conference, the United Nations World Water Assessment Programme published a report on Integrated Approaches to Water Resources Management (UNEP, 2012) to review the current state of IWRM application. 134 countries across the world responded to the survey in order to determine the progress towards sustainable water resources using integrated approaches. It was discovered that $82 \%$ of countries have embarked on reforms to improve the integrated approaches to water resources management. About $65 \%$ have already developed IWRM plans, while $34 \%$ are in an advance stage of implementation. $25 \%$ of participants still report obstacles in implementation due to weak or conflicting legal frameworks, and inadequate or non-existent strategic planning. 
In order to further stress the codependence between human society and natural resources, water in particular, Sivapalan (2012) suggests a new science with many parallels to IWRM. The new science is called socio-hydrology, and aims to study the dynamics and co-evolution of coupled human-water systems. In socio-hydrology, humans and their actions are seen as a part of the water-cycle dynamic, and this science aims to predict the dynamics of the whole system. Socio-hydrology is similar to eco-hydrology, a science that investigates the co-evolution and self-organization of vegetation in the landscape in relation to water availability. The main difference between IWRM and socio-hydrology is the study of humans and water in co-evolutionary systems. The main characteristic of these systems is that they consist of processes of generation of "new variations". New variations, or emergent behaviors, result from feedbacks between processes at different scales, through which systems can evolve into new states. Similarly to IWRM, Sivapalan (2012) suggests that socio-hydrology strives to be a quantitative science where quantitative descriptions are necessary for testing hypotheses, modelling the system, and predicting possible future trajectories of system states.

\subsection{Integrated Water Resources Management (IWRM) in Canada}

An integrated approach to management of natural resources has long been accepted by the Canadian administration. This chapter analyses the current management practices on the Canadian federal level and in Ontario. Finally, it explores the role of Ontario's Conservation Authorities in the water management process.

\subsubsection{Integrated Water Resources Management (IWRM) on the Canadian federal level}

Compared to most other parts of our planet, Canada has a plenitude of water and land resources. It is second only to Russia in terms of the total area occupied $\left(9,984,670 \mathrm{~km}^{2}\right)$. It is estimated that Canada holds $20 \%$ of the world's freshwater reserves, $7 \%$ of which is renewable, while its rivers discharge around $9 \%$ of the world's total renewable water supply (Statistics Canada, 2010a). Despite abundant water resources at the national level, however, there are regions in Canada that suffer from significant seasonal water 
shortages, frequent flooding events, or else have serious problems with the quality of local water supply. In addition to this are specific water problems arising from socioeconomic activities, resulting in poor water quality, reduced quantities, imbalances between supply and demand, and natural water-related hazards.

Floods and droughts are two of Canada's most costly natural threats. The expected effects of climate change will likely bring additional challenges. In order to address these challenges, it is estimated that the cost of water infrastructure development and maintenance will continue to grow (IISD, 2009). The Canadian water community warns of potential conflicts in the near future between competing users of water and land resources, such as agriculture and food industry, energy production, municipalities, industry, and recreation. As a response to an already experienced and projected population growth, the need for an integrated approach to management resources is recognized across the different levels of Canadian administration. IWRM is seen as a natural approach to addressing the stated challenges. If adapted to the local context, it offers ways to balance the social, economic, and environmental demands on water supplies. From the Canadian perspective, IWRM is considered to be "a multidisciplinary and iterative process that seeks to optimize the contribution of both aquatic and terrestrial resources to the social, environmental and economic welfare of Canadians, while maintaining the integrity of ecosystem health, both now and into the future" (IISD, 2009).

On the Canadian federal level, key elements of the IWRM strategy are incorporated through the Federal Water Policy of 1987. Due to the divided legislative jurisdictions between the federal and provincial/territorial authorities, the Canadian federal government has no formal mechanisms for coordinating and prioritizing the implementation of the IWRM process (Morin and Cantin, 2009). Canadian federal agencies and departments act independently, reflecting their respective roles and mandates. Currently, under the Constitution of Canada, water and environmental resources management is a shared responsibility between the federal, provincial/territorial and municipal governments. All three are steadily moving toward integrated ecosystem and watershed management based on the principles of sustainable development. These principles are created to ensure that the required decision making takes into account the 
interests of all stakeholders, and balances a whole set of goals: sustainable water, aquatic and land resource management, protection from health threats linked with water quality, protection of aquatic ecosystems and species, and the reduction of health, economic, and safety impacts from floods and droughts. Current federal regulations give the provinces and territories central authority to allocate the appropriate amounts of water to different users, and for different purposes. However, if their decisions have certain impacts on areas under federal responsibility, such as protection of fish habitats, the federal institutions of the Government of Canada need to be involved. There are 20 departments within the federal government that have responsibilities for water, while 8 have strong water-related mandates, (Morin and Cantin, 2009). Federal government responsibilities are:

- Provision of drinking water in areas of federal jurisdiction (First Nations, national parks, national defense);

- Aquatic ecosystem protection including fish habitats and those of species at risk;

- Marine navigation;

- Ensuring water availability for agricultural purposes, and formal agreements for allocating water resources between provinces, as well as between Canada and the U.S.

From a practical perspective, this divided approach may be inconsistent in terms of the federal involvement across Canada's watersheds, and can bring blurred messages from different federal authorities to local authorities. Morin and Cantin (2009) suggest changes in strategy for the federal government that could enhance the collaboration of departments and coordinate their activities. The new strategy should:

- Enable the federal government to prioritize its involvement to ensure it meets its own obligations;

- Accommodate the widely diverse watershed issues, tailoring solutions to specific circumstances;

- Guide the collaboration and coordination of federal departments with watershedbased non-governmental organizations; 
- Ensure the federal government's involvement is compatible with IWRM principles; and,

- Complement the different governance mechanisms for watersheds across Canada and those shared with the U.S.

\subsubsection{Integrated Water Resources Management (IWRM) in Ontario}

As the management of natural resources is a responsibility not only at the federal, but also the provincial/territorial level, each Canadian province/territory delegates responsibilities to the relevant institutions managing its own natural resources. In Ontario, The Ministry of Natural Resources and The Ministry of the Environment and Climate Change have central roles in managing the local natural/water resources. Both ministries have a goal to support the needs of local inhabitants and provide a healthy natural environment at all times, in collaboration with other provincial and federal institutions. However, the two ministries have distinctive roles in terms of water management. The Ministry of Natural Resources has the following responsibilities related to water management:

- Protection of human life, property and natural resources through forecasting and warning about flood/drought/erosion hazards, as well as overseeing the safety of water control structures, such as dams;

- Support the development of healthy local, regional, and provincial economies through sustainable use of water resources for activities, such as hydroelectric power generation and management of Crown-owned dams;

- Monitor the state of climate and surface water flows and levels;

- Ensure the integrated management of Ontario's water resources through water budgeting, river management and watershed planning;

- Safeguard Ontario's interests on shared boundary waters. 
On the other side, the Ontario's Ministry of the Environment and Climate Change water management activities are:

- Management, licensing, and testing of drinking water, as well as protection of drinking water sources;

- Management of water quality associated with the Great Lakes and inland lakes;

- Surface water and groundwater quality monitoring;

- Water taking permits;

- Well licensing and management;

- Promoting water conservation.

Despite the water management activities of the two ministries mentioned above, the most prominent role in promoting and implementing the principles of IWRM in Ontario belongs to the Conservation Authorities. Conservation Authorities (CA) were introduced in 1946 by the provincial government, and legally established by the Conservation Authorities Act (Conservation Ontario, 2014). This was a response to the growing concerns of the local environmentalists and the general population about the deteriorating state of the natural resources in the province. A series of detrimental flooding events led to the conclusion that natural disasters such as droughts and floods in Ontario were a result of uncoordinated land, water and forestry practices during the 1930s and "40s. In 1946, a number of municipal councils decided to get involved in managing local resources. Leading provincial authorities came to believe that a holistic approach to natural resources management was necessary on a watershed basis. This inter-municipal cooperation eventually led to the Conservation Authorities Act. The Act enabled the province and municipalities to form the Conservation Authorities within a watershed with the goal to establish programs of natural resources management. Key activities of the Conservation Authorities are:

- Environmental protection - protection of local ecosystems and contribution to the quality of life in communities throughout the province.

- Water resources management - promote integrated, ecologically sound environmental practices to manage Ontario's water resources on a watershed 
basis, maintain secure supplies of clean water, protect communities from flooding and contribute to municipal planning processes.

- Lifelong learning - support educational experiences in a natural environment that enrich the lives of people of all ages.

According to the Act, municipalities within a watershed share responsibilities and costs of water management activities, such as flood control, dam operation and maintenance, floodplain management, soil erosion, reforestation, recreation and education. Today, Conservation Ontario has 36 Conservation Authorities in all the major watersheds, which cover $90 \%$ of the province's population. Conservation Ontario sees IWRM as:

"the process of managing human activities and natural resources on a watershed basis. This approach allows us to protect important water resources, while at the same time addressing critical issues as the current and future impacts of rapid growth and climate change. This approach also allows us to address multiple issues and objectives and enables us to plan a very complex and uncertain environment." (Conservation Ontario, 2014)

In consequence, the main principles of IWRM are implemented by the provincial Conservation Authorities which have a mandate to "ensure the conservation, restoration, and responsible management of Ontario's water, land and natural habitats through programs that balance human, environmental, and economic needs" (Conservation Ontario, 2014). Conservation Authorities work with provincial partners to deliver consistent plans and practical solutions to numerous challenges in natural resource management. One of the most important outcomes of their activity are the watershed report cards. In 2013, 28 Ontario's Conservation Authorities produced and published the watershed report cards for the general public. These report cards serve as a means of evaluation, targeting priorities in the management of resources, measuring current states, and monitoring existing environmental change.

Experience has shown that the main governance model assigned to the conservation authorities blends top-down and bottom-up approaches by promoting dialogue and joint problem solving between stakeholders and the provincial and municipal governments. 
The usefulness of having Conservation Authorities was demonstrated when the Clean Water Act and Source Protection regulations were introduced by the provincial government (Mitchell et al., 2014). The Clean Water Act protects the quality of municipal drinking-water resources. The Source Protection regulations have established Committees that are responsible for addressing significant threats in the designated wellhead protection areas, surface water intake zones, and vulnerable recharge areas. Consequently, their assessment reports are scoped to identify and assess potential risks to the sources of drinking water. In addition, Mitchell et al. (2014) assess that the Ontario's Conservation Authorities have achieved noteworthy results in IWRM application. Despite a number of technical and organizational issues, the Conservation Authorities represent an innovative model of watershed-based management and source-water protection. The effectiveness of this approach is a result of a "collective mandate with measurable objectives, articulated roles, and responsibilities for all participants, capacity to obtain financial and human resources, and influence initiatives with water security implications".

\subsection{Systems approach to water resources management}

As previously established, the accepted definition of integrated water resources management, formulated by the Global Water Partnership (GWP), requires that technical as well as environmental, social, and economical aspects of the engineering solutions be properly analyzed and taken into account in daily practice. However, moving from generic definitions and ideals of IWRM to an effective implementation is a great challenge. Regular practices in water resources management involve a whole set of tasks which include: definition of appropriate operational policies; assessment of local, regional, and national resources; formulation and implementation of management strategies; planning, design, construction, maintenance and operation of assumed structures and facilities; regulatory and permitting functions; scientific and engineering research; and education and training of general public and interested parties. More specifically, engineering aspects of water management activities involve development, control, protection, regulation and beneficial use of surface and ground water resources, 
water supply for agriculture, industrial, and municipal use, wastewater collection and treatment, protection and enhancement of environmental resources, pollution prevention, recreation, navigation, hydroelectric power generation, stormwater drainage, erosion and sedimentation control, controlling flood water and reducing damages due to flooding (Wurbs, 1994). Having in mind the nature and complexity of these activities, Biswas (2004) analyzes the most often quoted GWP definition of IWRM and finds that this definition has very little practical resonance on present and future water management practices. Biswas (2004) argues that from the practical standpoint the definition is unimplementable since it does not provide clear guidance to water professionals. It remains a question how exactly the concept should be utilized to make the water resources management process more rational, efficient and equitable. For this reason, to bridge the gap between theory and practice, and make the IWRM concept more valid, Simonovic (2009b) offers seven guiding principles to pragmatically and scientifically guide all IWRM activities:

Systems view: since water affects significantly all terrestrial systems, the systems view principle recognizes the need to account for a broad set of relations among water and land resources, in order to ensure that critical relationships are recognized and managed.

Integration: water resources management regularly suffers from fragmentation of responsibilities between different institutional levels (local, provincial, national, or international). The purpose of this principle is thus to promote vertical integration of various levels of government dealing with water resources problems. Another problem addressed here is fragmentation within each level (horizontal fragmentation), e.g. different government agencies (agriculture, forestry, fisheries, mining, municipal affairs or economic development). This principle therefore fosters both vertical and horizontal integration through coordination and collaboration.

Partnership: vital importance of water resources requires that the objectives of water and land resources management be defined by individuals of diverse social, cultural and scientific backgrounds. The principle thus calls for a strong collaboration of the engineering, social, natural, ecological and economic sciences. 
Participation: this principle recognizes that water as a resource is a subject of everyone's interest. It ensures active involvement of all interested stakeholders, giving them authority to make decisions at different stages of water management. This participatory approach is designed to facilitate long-lasting consensus and common agreement.

Uncertainty: modifications of water bodies and lands by human activity fundamentally alter aquatic systems. Analysis of effects of potential modifications will therefore inevitably be based on incomplete information about many occurring processes. This leads to multiple uncertainties in the IWRM decision-making process.

Adaptation: a high degree of uncertainty highlights the need for adaptive IWRM, within which the relationships between planning and outcomes are explicit, and in a feedback loop. By building an understanding of the feedbacks and interactions taking place, adaptive decisions can be made. Embedded interactions between hydrology, land use, ecology, institutions, policies, and social interactions within a basin make an integrated approach to water management possible.

Science and data: IWRM requires involvement of different scientific domains, such as hydrology, hydraulics, geology, meteorology, oceanography, environmental science, engineering, law, economy, mathematics, etc.

While each guiding principle is equally important for the IWRM process, the systems perspective underlies them all. An elaborate process of coming up with an adequate solution requires a set of problem solving techniques found in systems analysis. Tools of system analysis integrate a set of techniques of analytical operations to procuring optimal solutions to problems of complex systems.

Before outlining the properties of complex systems, Simonovic (2009a) defines a system as a collection of various structural and non-structural elements that are connected and organized in order to achieve a specific objective through the control and distribution of material resources, energy and information. Meyers (2009) expands this definition from a social viewpoint, describing complex systems as systems that consist of many interacting parts with the ability to generate a new quality of collective behaviour through self- 
organization, i.e. the spontaneous formation of temporal, spatial or functional structures. Meyers further suggests that complex systems are adaptive as they evolve and may contain self-driving feedback loops. He concludes that complex systems are much more than a simple sum of their parts. The unique properties of all complex systems Meadows (2011) puts forward are: system elements, connections between them, connections between the elements and the environment, as well as function or purpose.

Within the framework of water resources management, the complexity of the underlying structure of natural and human-made systems has been realized only recently. According to Simonovic (2009a), a water resources management system is a result of the interaction of the following three main components, also known as sub-systems:

- The natural river subsystem in which physical, chemical, and biological processes take place;

- The socio-economic subsystem which include all human activities related to the use of the natural river and land systems;

- The administrative and institutional subsystem of administration, legislation and regulation, where the decision, planning and management processes take place.

The magnitude and complexity of the decision processes within water management practices require the most effective use of scientific and quantitative methods of system analysis. In order to analyze complex systems and find the optimal solutions, the systems analysis utilizes the following set of techniques, Simonovic (2009b):

1. Simulation;

2. Optimization;

3. Multi-objective analysis.

Computer simulations play an important role in all aspects of water management. The main role of computer models is to expand our understanding of real-world processes. From the perspective of the water community, computer models provide substantial 
information to support educated and informed decision-making. A simulation model represents a simplified but purposeful representation of a real-world system. The model must extract enough information out of the complex world to adequately formulate the studied problem, while spatial and temporal scale, as well as the structural complexity of the model, will strongly depend on the nature of the analyzed problem. In addition to simulation models, optimization is another mathematical modeling approach used in systems theory. While simulation is characterized as a "descriptive" approach, optimization is considered "prescriptive", as it formulates an optimal course of action, or else an optimal design for given conditions. Optimization models define the problem in terms of desired objectives, design variables, and problem constraints. Design variables are a set of parameters the optimal values of which are sought after (Simonovic, 2009b). Problem objectives are defined through objective function, and are used to determine the quality of a particular solution. Constraints represent physical, economic, social, environmental and all other restrictions. However, management of complex water resources systems rarely involves a single objective. Multi-objective optimization, or multi-criteria optimization, is spoken of when more than one objective function is to be optimized simultaneously. In contrast to single-objective optimization, optimal solutions are rarely possible for multi-objective problems, and trade-offs between different parts of the problem are inevitable. Value judgments then become an important part of the decision-making process.

\subsection{Problem statement}

In contrast to optimization and multi-objective analysis models, simulation models do not find optimal solutions to given problems, but rather provide quantitative and qualitative descriptions of a system's structure, replicating - in simplified form - its behavior in time and space (Loucks and van Beek, 2005). The system's behavior in response to different conditions is constructed using a set of "what-if" questions. Water resources systems (WRS) are one of the most complex systems to model because of the interdependence of multiple physical, bio-chemical, social, legal and political processes that influence and drive their performance. The different aspects of complexity of WRS are listed below: 
1. Complex objectives - a direct consequence of the simultaneous use of water by different users, the need for protection of water quality on the one hand, and detrimental effects of water on the other;

2. Opposing interests - WRS regularly include a great range of conflicts between different system users;

3. Complex dynamics - WRS are dynamic systems where management in the past affects the system performance in the future, there are constant changes in system functionality, and continuous development and modification of certain elements of the system;

4. Asynchronous properties - in typical WRS, the highest water demand is observed in periods when the system inflow is lowest, and vice-versa;

5. Environmental aspects - WRS crucially influence the current state and future development of the neighboring environment;

6. Social aspects - WRS can initiate enormous social/demographic changes;

7. Stochastic nature - WRS are characterized by a whole set of uncertainties, starting with system inputs, demanded system outputs, current system states, system objectives, limitations, etc.;

8. Spatial variability - WRS are the largest systems humans build and manage. Incorporation of other components (e.g. social-economic) makes these systems even larger and more complex.

The process of model development requires an extensive knowledge of the system being analyzed, identification of model objectives, significant amounts of data, and solid analytical and programming skills. The most important step in the modeling stage, however, is the selection of an appropriate modeling method. Traditional modeling methods offer different advantages in representing different aspects of complexity of water resources systems. Their ability to adequately represent all eight aspects of complexity varies. The main shortcoming of traditional modeling methods, e.g. System 
Dynamics (SD) simulation, is their inability to capture both spatial and temporal dynamics of water resources problems at the same time.

It is therefore necessary to investigate existing modeling methods and their unique advantages and disadvantages in representing both temporal and spatial scales of water resources systems. This is the object of our present research. The goal is to construct a generic multi-method modeling framework, the main objective of which is to capture all structural complexities and interactions inside water resources systems, placing special attention on including both spatial and temporal variability.

\subsubsection{System Dynamics (SD) modeling method}

System dynamics (SD) simulation is one of the modeling methods frequently used in IWRM due to its capacity to describe complex relations between all three subsystems of water resources management systems. It utilizes the principles of the feedback control theory to shape computer models, and the feedback loop presents the core building block of this approach (Prodanovic, 2007). According to Meadows (2008), system dynamics modelling methodology starts with a premise that the structure of the system lies at the root of its behavior, implying that any change to system structure will alter system behavior. SD simulation is a modeling method introduced by Forrester in the late 1950s (Forrester, 1961). It focuses on the structural complexity of a system and its dynamic behavior. Forrester originally used this methodology to analyze the demand amplification effect, known as "Forrester Effect", in supply chains analysis. Later, he applied it to analysis of urban and world development (Forrester, 1991). Since its introduction, the SD modeling methodology has been applied in a number of scientific domains, such as economics, software development, environmental sciences, economic markets and competition analysis, force management, etc. From the water resources management perspective, it offers a number of advantages. Firstly, it allows integration of social, economic and environmental elements into the equation. Secondly, it provides a direct link between the structure and the behavior of a system, making further examination of the change in system structure and its effects on system behavior possible. Finally, it promotes an active involvement of all interested parties and stakeholders in the modeling process, in tune with the principles of IWRM. 
In SD simulation, a complex system is represented as a combination of seven basic elements:

1. Sources;

2. Flows (Inflow and Outflow);

3. Stocks;

4. Sinks;

5. Variables;

6. Feedback loops.

Each element has its own set of mathematical equations, and the purpose of the simulation is to discover how the whole system reaches equilibrium. The complexity of the system is a result of non-linear and multi-loop feedbacks, thus the system structure remains the main driver of the complexity (Sterman, 2000). In a widely-used system dynamics simulation software the simulation elements are depicted as graphical objects, and Figure 1 shows the graphical representation of the main elements.

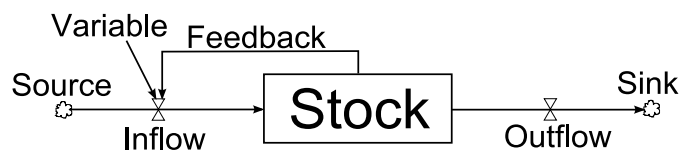

Figure 1: Main elements of system dynamics simulation

Stocks and flows represent the core of all SD models. The stocks can be described as accumulations, but, in a mathematical sense, they are simply integral equations. These accumulations drive the model toward equilibrium as they stabilize the whole system (Lättilä et al., 2010).

The SD modeling method has been applied to a variety of water resources management (WRM) problems, as for instance: drought management studies by Keyes and Palmer (1993); management of scarce water resources by Fletcher (1998); reservoir operation for flood control and management by Ahmad and Simonovic (2000a, b, c); hydrologic studies under climate change by Simonovic and Li (2003) and others. Winz et al. (2008) give a wide overview of the use of SD modeling methodology in water resources 
management, finding that this method has been successfully applied to problems in regional planning and river basin management, urban water management, flooding and irrigation, especially when combined with stakeholder involvement. Tidwell et al. (2004) use the method to describe a community-based planning project in the Middle Rio Grande river basin. This semi-arid region faces the challenge of balancing a limited water supply with increasing demands due to population growth and the associated urban development, irrigation and water abstraction for riparian/in-stream use. Stave (2003) applies the method to an urban water management project in Las Vegas, USA. Urban water demand was projected to exceed supply by 2025, so the local Water Authorities needed to convince the public of the need for water conservation. Ford (1996) develops a Snake River Explorer model to simulate the annual flows of the Snake River, USA. The river suffers from over-appropriation, low river flows and declining water tables that threaten agriculture and aquaculture industries, rural livelihoods and ecological diversity in the area. Hassanzadeh et al. (2014) use the method to develop an integrated water scenario analysis of the Saskatchewan portion of the transboundary Saskatchewan River Basin. This expanded the existing WRM model by introducing an irrigation sub-model to account for the dynamic irrigation demand, as well as an economic sub-model that provides an economic perspective of water use for various sectors. Dawadi et al. (2012) study effects of climate variability and climate change on the Colorado River flows, as well as their implications on local water resources management.

\subsubsection{Limitations of the System Dynamics (SD) modeling method}

Despite its wide application to water resources management, the SD modeling method has a number of important limitations. In order to evaluate the risk and vulnerability of the Upper Thames River basin in Ontario from the changing climate and socio-economic conditions, Prodanovic and Simonovic (2010) use SD simulation to capture the physical and socio-economic processes that take place within a basin, and to analyze their interdependence. The model produced consists of two components, a physical processbased model (in this case a semi-distributed hydrologic model), and a model that describes the relevant socio-economic processes on the basin level. Prodanovic and Simonovic (2010) therefore combine the two modeling methods recognizing the first 
limitation of the SD modeling method - representation of complex spatially distributed physical, chemical and biological processes. In addition, Roach and Tidwell (2009) compare a process-based Albuquerque basin ground water dynamics model, developed in MODFLOW, with the compartmental spatial system dynamics (CSSD) model. Based on the comparative analysis of the respective results, they conclude that CSSD is not an appropriate tool for problems requiring a detailed analysis of ground water dynamics and, like Prodanovic and Simonovic (2010), they give an advantage to traditional processbased modeling methods.

Prodanovic (2007) further recognizes that the SD models, especially those representing complex social systems, are imprecise and do not give accurate quantitative predictions. These models also require extensive data for calibration and/or verification purposes. He also notices that the SD approach often results in oversimplified and largely aggregated system representation. Many key socio-economic processes occur on much finer spatial and temporal scales, and modeling at high levels of aggregation can potentially fail to capture important system features. In practice, oversimplification and aggregation can be overcome by initializing the model in a most general and aggregated form. Once this higher level has been studied, additional system details can be introduced. According to Prodanovic (2007), however, experience has shown that further details introduced to SD models do not necessarily bring additional objectivity and accuracy to the drawn conclusions.

But perhaps the most important limitation of the SD modeling method is that it does not explicitly represent spatial system elements and spatial variability within a modelled system. As the use of the method became widespread over the last 40 years, and the range of application expanded over time, a number of significant attempts to introduce the spatial component to SD modeling has been made (BenDor and Kaza, 2011). The authors suggest that spatially explicit modeling uses disaggregated spatial data and relationships in order to understand spatial forms and processes. "Spatialized" SD models should explicitly simulate the system structure that is normally heterogeneous over space, and observe how spatial interactions affect system behavior. 
There have been a number of theoretical platforms and system archetypes built to represent dynamic systems whose structure and behavior are determined by spatial processes. Selecting an appropriate system archetype is of crucial importance, as is defining the spatial and temporal scales of a SD model, since different approaches of representing space (zonal, grid or network) completely alter the structure, composition and behavior of spatial models.

Zonal models are mainly applied to studies of urban dynamics. They disaggregate space, in this case urban spaces such as cities, into distinctive zones which represent distributed properties of the system (central business districts, suburbs, etc.). However, Anselin (2002) concludes that zonal models present a restricted solution in cases when significant environmental and spatial system heterogeneity determines global system behavior.

One recent development in the spatial-system dynamics research is a tessellation of space into regular grids (BenDor and Metcalf, 2006). Ford (1999) used a gridded landscape to represent heterogeneity in a drainage basin, where stocks represent water levels in connected areas, only to find laborious difficulty in replicating an SD model for each grid cell, a limitation similar to the one observed in zonal models.

As a solution to this problem, Ahmad (2002) suggests an innovative approach to modelling feedback-based dynamic processes in both time and space, naming it Spatial System Dynamics (SSD). He develops the model by coupling the SD model to the Geographic Information System (GIS) through a data exchange link. SSD requires that the area of interest be divided into cells. Each cell contains an SD model which communicates with cells through a set of feedback links. This interaction initiates a change in the observed parameters at any point in space according to an average of the parameter values in neighboring cells. Neuwirth et al. (2014) take a similar approach and create a Python program to tightly couple SD software to a GIS component. This approach achieves the required capacities for bidirectional interactions of operations between SD and GIS. They discover that the results of the spatial model developed for the case study are not only influenced by the initial spatial structure, but also depend on raster resolution. Anselin et al. (2002) and Vionov (2007) show that both processes are 
greatly exposed to artifacts of grid geometry, which is likely to go undetected in SD modeling. Performance of the sensitivity analysis can thus become extremely difficult, especially in cases where spatial data is available at a low spatial resolution.

Finally, BenDor and Kaza (2011) suggest that spatial interactions can be observed across a topological network that defines the underlying structure of space. This approach articulates space through weighted networks (links and nodes). They go on to show that this approach would allow for the departure from an arbitrary gridded representation of space. A more rigorous analysis of how models are affected by particular spatial representation would thus be made possible. The network representation of space treats spatial relationships as fully dynamic and allows changes in the local spatial structure which affect the dynamics of global processes.

Another difficulty in bringing the spatial component to SD models is visualization. BenDor and Kaza (2011) recognize a number of spatial archetypal behaviors of extensive processes and their potential modes of visualization (Liner Growth, Exponential Growth, Goal-seeking Growth, Logistic Growth, Sustained Oscillations, Overshoot and Collapse), concluding that such visual representation is not an appropriate solution for more complex and intensive processes.

\subsubsection{Alternative modeling methodologies}

Beside the SD simulation, a number of other modeling methods are available for describing the feedback-based processes in both time and space, in particular Cellular Automata (CA), Temporal GIS, and Agent-Based Models (ABM).

\subsubsection{Cellular Automata (CA)}

Cellular Automata (CA) can be described as a discrete dynamic system modeling method. This modeling method analyzes the global system behavior as a result of local interactions. The CA approach divides the space into cells (automata) of the same size and shape, usually square. Cells can also be triangular or hexagonal, depending on the requirements of the problem under consideration. The complex global system behavior emerges as a result of local interactions between individual cells. System behavior is 
therefore dynamic both spatially and temporarily. Each cell holds a value of either 0 or 1 (Dragicevic, 2010). The state and behavior of each cell are determined by the states of other cells in close vicinity at a previous time step. Interactions between cells are determined by a set of local rules.

The main elements of Cellular Automata are:

- The cells in a regular grid;

- Cell states, $S$;

- Neighborhood of the, $N$;

- Function of cell transition rules, $R$;

- And discrete time increments, $\Delta T$.

The cell state $S_{(x, y)}$ at time $T$ can be described as function $F$ that depends on CA elements at previous time $\mathrm{T}-1$ and can be formalized as:

$$
S_{(x, y) T}=F\left[S_{(x, y) T-1}, N_{T-1}, R_{T-1}, \Delta T\right]
$$

where $S_{(x, y) T-1}$ is the cell state at location $(\mathrm{x}, \mathrm{y})$.

Each discrete time step is the CA model iteration. In each time step, transition rules and the state of other cells in its local neighborhood update the state of each cell in the grid. CA modeling method uses the various types of neighborhoods: The Von Neumann, Moore, Extended Moore, Displaced Von Neumann, Moore Von Neumann, and H Neighborhoods. The neighborhoods can take different shapes and sizes, such as symmetric, asymmetric, rectangular, and circular shapes. The transition rules define the way in which the cell develops in time, and therefore replicates the overall change process of the cells over space and time. The rules can be deterministic, probabilistic, stochastic or fuzzy.

Since the global behavior emerges from an application of local rules, CA is appropriate for simulating systems where global processes are not fully understood, but for which local interactions are known. One of its drawbacks is the fixed position of the cells; they can only change their state; their position and relationships remain fixed over time. 
Another limitation is the fact that cells cannot store more than one attribute (Ahmad, 2002).

The CA modeling method has been applied to various scientific domains, such as medicine, chemistry, ecology, biology, geography, urban planning and so on. In geographical studies, it has been applied to land use change and urban growth studies at different spatial scales (Dragicevic, 2010). Combining well with advances made in satellite data collections and raster geographic information systems (GIS), the CA modeling method has been growing in usefulness. The integration of CA and GIS makes the representation of both spatial and temporal system components possible. However, certain practitioners in the field argue that the CA models are suitable for analysis only, not for prediction, as they are based on a particular model design or data, and it is difficult to modify them for new studies (Dragicevic, 2010).

This method has also been applied to different aspects of water resources management. A recent application is in the area of urban flash floods analysis. Ghimire et al. (2013) couple one-dimensional hydraulic model and a CA model to represent two-dimensional space. This CA model uses regular grid cells as a discrete space and applies generic rules to local neighborhood cells in order to simulate the spatio-temporal propagation of pluvial flooding. The proposed CA model is applied to a hypothetical terrain and a real urban area.

Despite a number of suitable features, from a purely water resources management perspective, the CA modelling method comes with certain limitations. First, it is difficult to describe spatial phenomena where global processes and variables influence system behavior. Second, the transition rules of local interactions do not change during the simulation time. This represents a serious drawback for application to water resources management, as the transition rules rarely remain constant over time.

\subsubsection{Temporal GIS}

The traditional Geographic Information System (GIS) facilitates management, analysis, and representation of spatial information. This approach is designed to statically represent 
time-dependent processes and is incapable of representing continuous changes within the model structure. Temporal GIS, also known as spatio-temporal GIS, expands this method by introducing the temporal analyses. Temporal GISs takes a number of different ways to achieve this.

The time-stamping approach adds temporal information to defined geographic entities. (Yu, 2010). A single time-stamp can be an attribute of a geographic layer, where all geographic entities in the layer share the same temporal information. It can also be an attribute of each single geographic entity separately containing its unique temporal information. The time-stamping methods are used to represent temporal changes of geographic entities, and to enable spatio-temporal queries and analysis.

The snapshot model represents an alternative to the time-stamping approach. It uses a collection of snapshots to demonstrate the change of geographic entities in time. Finally, a space-time composite model records accumulated changes in a single composite layer. The main limitation of these approaches is that they are incapable of a continual representation of time. Discrete changes only are considered.

Both approaches to temporal GIS face the challenge of capturing the driving mechanisms underlying the discrete changes. This is why the representation of dynamics processes remains an issue for temporal GIS (Yu, 2010).

\subsubsection{Agent-based $(A B)$ modeling}

Agent-based models (ABMs) are used to represent complex systems composed of a community of heterogeneous entities (also known as agents) spatially distributed over a shared environment (Ligmann-Zielinska, 2010). Agents are seen as autonomous entities that perform desired actions in order to achieve prescribed goals. Agents are also equipped with a set of rules that drive their behavior. Typical agent-based models have two main components: agents themselves and their environment. The components are coupled through systematic specification of interdependencies and feedbacks (Heppenstall, 2012). Depending on how they treat the environment, agent-based models can be divided into two categories: spatially explicit and spatially implicit models. 
Spatially explicit models consider space as an integral component of the system, and are used to establish feedback links between the agents and the environment. These models represent the spatial implications of social processes using the function of space as an attribute of both agents and the environment. In models representing the physical characteristics of the environment in great detail, the agents respond to the attributes of the landscape (e.g. physical barriers, soil type, infrastructure, or aesthetic qualities) by adapting their behavior to the features of the modelled environment (Heppenstall, 2012).

The majority of spatially explicit ABMs are based on a regular cell framework used for representing the environment. This approach is borrowed from the CA modeling method. However, while the CA models can be described entirely through interactions of spatial elements and phenomena, they do not offer support for the so-called actor-based processes. As a result, CA relies on stationary interaction topology, whereas interactions in ABMs can be changed dynamically, defined as they are at the level of agents (Ligmann-Zielinska, 2010). Compared to the CA modeling method, agent-based models allow for agents' actions to influence and be influenced by drivers physically situated all over the spatial environment.

The $\mathrm{AB}$ modeling method has been applied to various domains that deal with spatial processes, such as urban growth and sprawl, deforestation and reforestation, traffic congestion, and natural resources management. The method brings new perspectives to complex water resources management systems modeling despite its main challenges, such as difficulties with construction of proper cognitive models that adequately represent decision making, extensive data requirements, and delicate parameterization.

Despite the challenges mentioned above, the agent-based modeling method is recognized as an appropriate technique for representing complex interactions between human society and the environment. For instance, in order to represent the interactions between human and natural systems, and describe how they influence each other, Filatova et al. (2009) couple an agent-based model of human behavior that drives land-use/land-cover change and vegetation-cover alterations, with an ecosystem model of landscape carbon balance in low-density human-dominated landscapes. 


\section{Agent-based simulation in water resources management}

From a water resources management perspective, the agent-based modeling method has the potential to help in better understanding the complexity of interactions among water users, between water users and water resources, and the effects of human society on the environment. Recently, the agent-based modeling method has found numerous applications in the analysis of different aspects of water resources management. Some models study purely physical systems, e.g. spatial and temporal variability of water resources and their dependence on underlying hydrologic processes, while others examine the social aspects and complex interactions between water resources and water users. In some cases, developed agent-based models take an additional step and incorporate other methods of systems analysis, such as optimization and multi-criteria analysis. For instance, in order to address issues related to natural resources management in Northern Thailand, Becu et. al. (2003) developed an agent-based model (CATCHSCAPE) that simulates the dominant features of the basin and also replicates the decision-making process of farmers. The physical component consists of a hydrological system represented by simplified distributed water balance equations, existing irrigation schemes, and crop and vegetation dynamics, while the social component is described using a set of resource management processes and emerging policies from negotiations between involved stakeholders. Saqali et al. (2010) developed a decision support model (MAELIA) to assist decision makers with investigating potential social conflicts related to the water resources management in the Adour-Garonne river basin. This model uses the agent-based modeling method to recognize different entities involved in the water management process. It describes the water monitoring institutions and water users as agents and replicates their interaction within a basin. In the next example, in order to secure a healthy flow of the Yellow River, the Government of China introduced the quota-based Water Allocation Agreement in 1999. Since those measures haven't

produced the expected results, Cai et al. (2011) use a multi-agent system modeling framework to explore the different management options and their respective effects on irrigators and the downstream ecosystem. In addition, Yang et al. (2009) observe a basin as a multi-agent system composed of water users and the common environment from which they exploit the resource. In order to optimize the behavior of agents, they use a 
decentralized optimization method known as constraint-based reasoning. This bottom-up approach takes the objectives of individual agents into account, balancing them through interactions among the agents using a bargaining scheme. Van Oel (2009) uses an agentbased modeling method to analyze the processes related to the distribution of water availability over space and time. The model developed represents the local farmers as water users who respond to alternating spatial and temporal distribution of the water resource in a semi-arid basin located in Northeast Brazil. Valkering and Offermans (2009) develop a modeling approach that integrates the effects of the dynamics of water management practices and respective cultural and behavioral change dependent on alterations in the water resources systems. This modelling approach is presented in the form of an interactive computer game where the main goal is to explore future pathways of water management in the Ebro River Bain in Spain. Moreover, Akhbari and Grigg (2013) analyze the increasing competition for water use and resulting conflicts among competing interests between different users. For that purpose, they develop a new approach, modeled as a game, that uses agent-based modeling to simulate the behavior and interactions of the parties participating in a conflict scenario. To practically implement the modeling framework, they consider the water issues of California's Sacramento San Joaquin Delta region.

Analysis of the existing models shows that agent-based models are not only capable of representing the spatial variability of water resources systems, but this modeling method also includes representation of other useful aspects of complex systems directly applicable of water resources systems - heterogeneity of system actors and environment, dynamic feedbacks both among the system actors and between the system actors and the environment, and, finally, system disaggregation and bottom-up system representation.

\subsubsection{Hybrid multi-method models}

In response to the basic principles of IWRM, a number of models have been developed to integrate the various tools of systems analysis. They generally combine a simulation of the physical component of the system with analytic tools, as well as optimization and multi-objective techniques that describe and quantify regional socio-economic conditions. Cai et al. (2003) analyze the use of specific sustainability criteria incorporated 
into a long-term optimization model of a river basin. This model targets water-supply risk minimization, spatial/temporal equity of water allocation, and economic efficiency of infrastructure development. In order to achieve optimal WRM practice, short-term decisions are guided by long-term plans based on sustainability criteria. Ward et al. (2006) directly integrate physical components with economic water-related benefits expressed using a quadratic objective function to determine optimal water use, while Mainuddin et al. (2007) develop a coupled hydrologic-economic spreadsheet model that analyzes water allocation between different sectors under alternative policy scenarios. The resulting model optimizes profit and water allocation subject to hydrologic and economic constraints as defined by the policy scenarios. Raymond et al. (2012) recognize that accurate prediction of pollutant loadings is crucial for determining operative water management strategies, and use artificial neural networks as predictors of the nutrient load in a watershed. Clavel et al. (2012) use integrated models and information systems to assess the land-use visions of various stakeholders using their own evaluation criteria, while Coelho et al. (2012) develop a tool in support of IWRM which integrates three components (GIS, Fuzzy set theory, and dynamic programming optimization algorithm) to delineate homogeneous regions in terms of hydrography, physical environment, socioeconomy, policy and administration.

Due to the complexity of water resources systems, a modeler needs to be aware of all the advantages and limitations of different modeling methods. Depending on the level of complexity of a given problem, one frequently needs to combine a set of modelling methods to avoid their limitations. A number of examples combine various modelling methods in so-called multi-method hybrid models. For instance, Prodanovic and Simonovic (2010) developed an IWRM model in order to test the response of a system to changing climate conditions. This model couples the dynamics of physical (hydrologic) and socio-economic processes utilizing the advantages of system dynamics simulation. However, while the model manages to adequately represent temporal dynamics of a WR system, it is unable to describe the changes in the spatial structure of the system. In order to analyze the effects of contamination of the water distribution system on public health, Zechman (2011) develops a multi-agent modelling framework to simulate a contamination event under the effects given by utility managers and users. This multi- 
method modeling framework, used to assess alternative risk mitigation options and management strategies, combines agent-based, mechanistic and dynamic modeling methods. The agent-based model and the water system distribution model are integrated to simulate the dynamic hydraulic impacts of the agent's decision on the water distribution network.

\subsection{Research contributions}

The pressures affecting most water resources include population growth, changes in landuse patterns and the effects of climatic change. The present research analyzes the stresses that water resources are exposed to by developing approaches for more effective water resources management. It is based on the Integrated Water Resources Management (IWRM) paradigm that recognizes the need for coordination in the development and management of water, land and related resources to maximize economic and social welfare in an equitable manner without compromising ecosystem sustainability. According to the definition, IWRM deals with planning, design and operation of complex systems in order to control the quantity, quality, and the temporal and spatial distribution of water, with the main objective of meeting human and ecological needs and providing protection from water disasters.

This research examines the role of simulation in integrated water resources management process, and analyses the specific advantages and limitations of existing modelling methods. As a result, it defines the architecture of a generic multi-method modelling framework which aims to represent all the structural complexities and interactions within a water resources system. As the traditional modelling methods have shown to be inadequate, a multi-method modelling framework is necessary for implementation of the IWRM principles (systems view, integration, partnerships, participation, uncertainty, adaptation and reliance on strong science and reliable data). The proposed research adopts the multi-method simulation approach to address the interconnectedness and important feedbacks that are characteristic for water resources systems. The methodology developed in this research is designed to provide the support for IWRM through agentbased simulation. Emphasis is placed on explicit modelling and simulation of the key aspects of the complexity of water resources systems, including: 
1. Feedback based system structure;

2. Integral representation of physiographic, environmental and socio-economic subsystems, and their non-linear interactions;

3. Explicit representation of complex spatial and temporal scales of water resources systems;

4. And provision of support for direct stakeholder participation and involvement.

The implementation of this multi-method modelling approach should result in efficient and effective management of water resources systems both now and in the future. Based on the architecture of the generic multi-method modelling framework, an operational model for the Upper Thames River basin (UTRB), Southwestern Ontario, Canada, is developed in cooperation with local conservation authorities (UTRCA). The main focus is placed on description of complex interactions between local natural resources and the socio-economic environment. One of the main contributions of this research is the analytical interpretation of complex results that should enable responsible conservation authorities to design policies to protect, conserve, and manage local surface water resources. 


\section{Chapter 3}

\section{Methodology}

The primary objective of this section is to introduce the three levels of development of a new methodology designed to support integrated water resources management process through simulation. The new methodology is primarily planned to represent the complex interactions between all relevant sub-systems in a typical water resources system. Also, the methodology is intended to capture all aspects of WRS complexity, with the special focus placed on the explicit description of temporal and spatial variability of water resources systems.

On the first level, the global architecture of a generic multi-method modeling framework is developed based on the three different sets of requirements. Rather than being developed for a specific water management problem and water resources system, the generic multi-method modeling framework is designed to be applied to any problem that can take place in systems of different structures, locations and systems that are placed in different socio-economic and environmental contexts. On the second level, the more detailed architecture of a specific operational model for support of integrated water resources management is described. In this case, the term "an operational model" implies that the developed model is applied, documented and tested for a very specific system or a case study (Wurbs, 1994). Finally, the third level gives detailed logical and mathematical background of all components used to define the operational model.

\subsection{Level 1: Architecture of a generic multi-method modeling framework}

In order to properly design the architecture of a generic multi-method modeling framework, the following set of requirements is taken under consideration:

1. Modeling requirements in the context of integrated water resources management;

2. Requirements of the integrated water resources management (IWRM) process;

3. Requirements for addressing complexity of water resources systems (WRS). 
In the last several decades, due to the complexities of water resources systems and far reaching social, economic and environmental effects of engineering and administrative solutions, computer models have become a stepping stone in almost every water resources management process. According to Karamouz et al. (2003), a water resources management process is divided into 5 distinct stages, Figure 2:

1. Problem definition and data collection and processing;

2. Modeling stage;

3. Decision making stage;

4. Implementation stage;

5. Continuous monitoring stage.

After gathering all the necessary information and clearly defining the problem (Stage 1), simulation and optimization models are used in the modeling stage for finding possible alternative solutions (Stage 2). In the next phase, conflict resolution and multi-objective analysis are used to compare different alternatives. As seen in this figure, the selection of feasible alternatives, in many cases, depends on social and economic issues related to institutional framework and distribution of financial resources. Political issues may also influence the decisions if policy makers do not effectively participate in the planning process (Stage 3). After the final decision has been reached, the implementation phase implements the solutions (Stage 4). In the last phase (Stage 5), monitoring, evaluation and feedback to decision makers are carried out in order to adapt the planning schemes and the operating processes.

Additionally, a modeling stage of water resources management includes a number of distinct steps that must be taken, Figure 2 (Stage 2). 


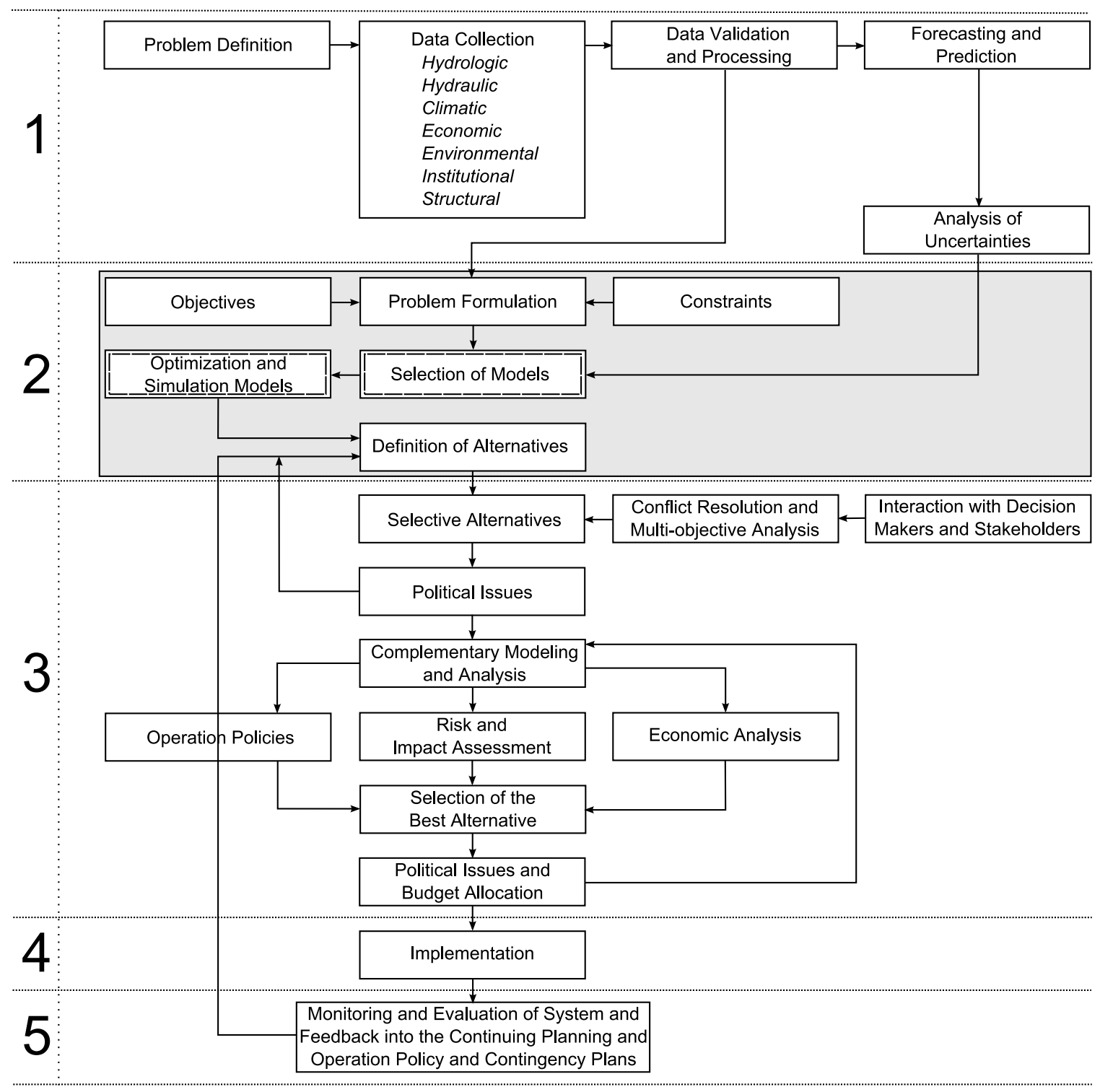

Figure 2: Modeling stage (2) in water resources management process

Prior to the actual model development phase, a model developer needs to properly analyze and formulate the existing problem. After the problem has been formulated, a modeler is required to collect, store and process all necessary data. For this reason, a database must be created to store and manage all relevant information. This stage also identifies the set of problem constraints and clearly defines the model objectives. Based on this, a modeler selects the most appropriate set of models to describe physical, chemical or environmental processes related to water management, such as water distribution systems models, models that describe groundwater dynamics and quality, 
watershed runoff models, stream hydraulics models, river and reservoir water quality models, or reservoir/river system operation models. A coupling of different models and their data exchange is often required to capture the scale and structural complexity of the water resources management problems. Therefore, responses to the requirements of the modeling stage are:

- A database to store and manage all relevant information and data; and

- A set of process-based models to describe physical, chemical or environmental processes.

According to the definition of IWRM and in order to support the systems view principle, the most important requirement of a newly designed modeling framework is an authentic representation of the interactions between natural resources and the socio-economic environment. In studies that include diverse social and large spatial scales, social and economic processes can be represented on different levels of abstraction - system (also known as aggregated) level and more specific individual-centric (or disaggregated) level. Depending on the scale, the framework allows representation of both levels by using one of two, or possibly both, modeling methods: system dynamics simulation for the system level and/or agent-based models for the individual-centric level. Consequently, responses to the requirements of the integrated water resources management process are:

- System dynamics simulation for representation of socio-economic processes at the system level (aggregated); and

- Agent-based model for representation of socio-economic processes at the individual-centric level (disaggregated).

In addition to the requirements of both the modeling stage and the integrated approach to water resources management, the main requirement of suggested architecture is authentic representation of the key aspects of complex water resources systems:

- Definition of a feedback based system structure between system elements;

- Integral representation of physical, socio-economic, and environmental processes; 
- Analysis of structural complexities and variability of different spatial and temporal scales; and

- Promotion of active stakeholder participation and involvement.

Based on previously listed requirements the proposed framework developed in this research contains a spatial database as the core component, Component 1, Figure 3. This database stores both spatial and non-spatial information required in the modeling process and directly provides necessary information to other components present in the system.

Component 2 includes a set of traditional process-based models used to describe the physical, chemical and biological environments. Traditionally applied process-based computer models in water resources management are water distribution system models, groundwater dynamics and quality models, watershed runoff models, stream hydraulics models, river and reservoir water quality models, and, finally, reservoir and river system operation models. Depending on the particular problem being analyzed, a modeler selects which process-based model should be used in the system.

On the other side, different characteristics of socio-economic environment are represented using Agent-based (AB) (individual-centric) and/or System Dynamics (SD) simulation (system level) modeling methods, Component 3 and/or Component 4 in Figure 3. While system dynamics simulation presents a well-recognized and explored methodology for describing the dynamic behavior of a complex system, agent-based simulation represents a relatively new modeling methodology based on the distributed artificial intelligence. The main idea behind multi-agent models is to build virtual complex system representations composed of autonomous agents, or identified system entities, which operate on a local knowledge, possess limited abilities, affect and are affected by the local environment, and thus enact the desired global system behavior. Agent-based models offer a number of ways to model processes at various spatial and temporal scales, and this makes them perfectly suitable for the implementation in water resources systems management. 


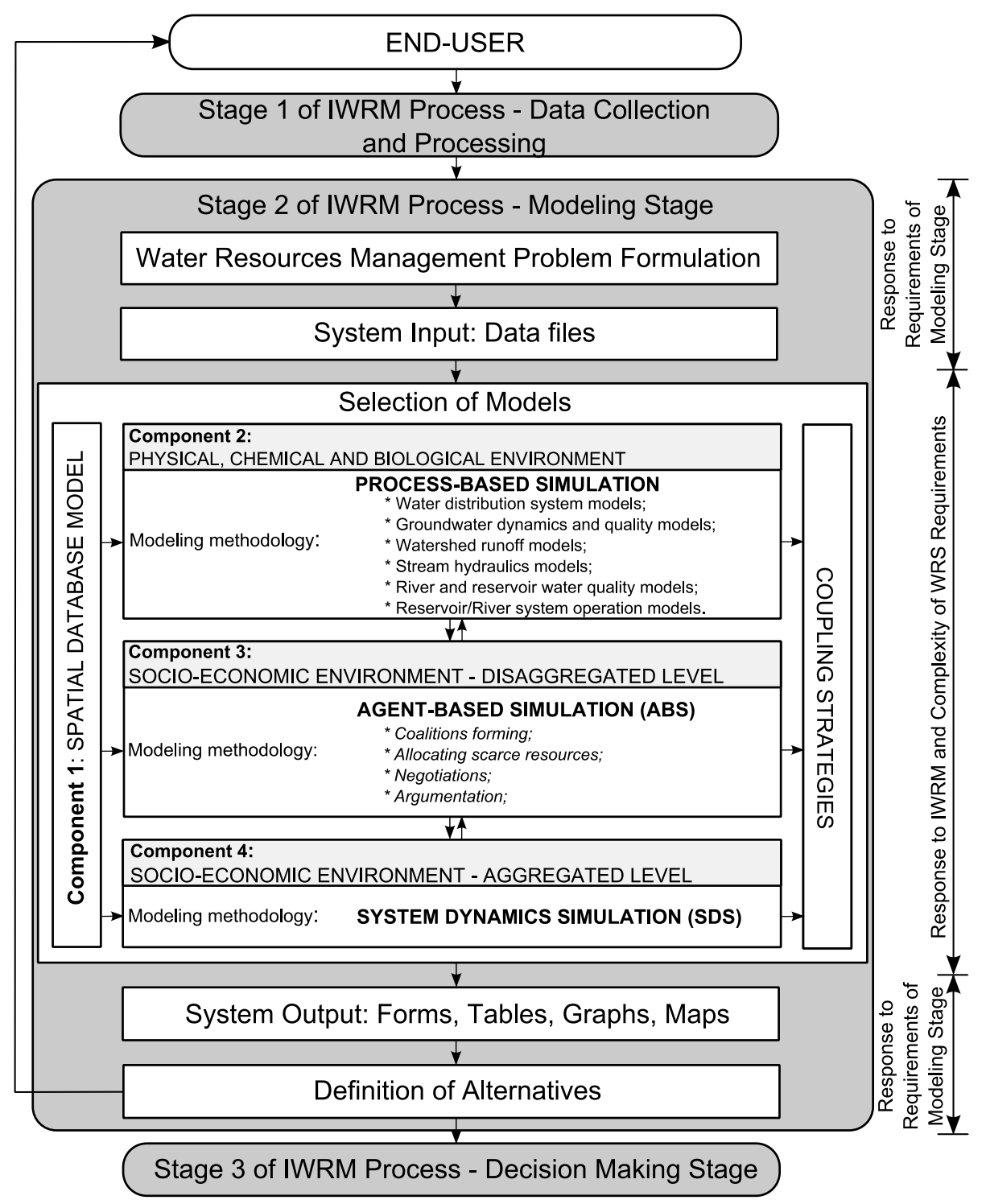

Figure 3: Architecture of the generic multi-method modeling framework

Designed generic modeling framework allows integration of components by choosing one of three available coupling methods: embedded; tight; or loose. The framework uses a set of output maps, tables, and figures to present the obtained results. Finally, the multimethod modeling framework allows utilization of simulation scenarios. Scenarios can be used to answer various what/if water management questions as well as to validate the results. In the case of integrated water resources management, scenarios can be used to assess the impact of changing land use practices, planned water use, and the hydrologic effects of climate change. 


\subsection{Level 2: Detailed architecture of an operational model for support of integrated water resources management}

This section details the architecture of an operational model to support integrated water resources management. The methodology is applied to describe the interactions between physical and socio-economic environments, Figure 4. The detailed architecture is developed according to the requirements of the designed generic multi-method modeling framework.

According to the basic principles of integrated water resources management, physical (in this case hydrologic) and socio-economic systems depend on one another and continuously influence each other through a number of feedback processes. Analysis of interactions between those two systems is typically built upon the conceptual representation of water balance or water budget models. This places the spatially semidistributed model in the center of the multi-method modeling system, Component 2 in Figure 4. Based on the climatic inputs (precipitation and temperature) and current physical conditions in a basin, the hydrologic model provides information on stream flows, evaporation and groundwater recharge rates. After hydrologic models estimate the hydrologic quantities, they are typically compared with the water demand. This value, on the other hand, comes from the disaggregated spatially explicit socio-economic model, Component 3. Estimates of water demand come from the socio-economic sub-models that, among others, include population dynamics, industrial and agricultural production, economic activities, urbanization, reforestation and deforestation. Component 1, the spatial database, stores and supplies all data relevant to the socio-economic model. Also,

the hydrologic model takes into account temporal and spatial alterations of physical and climate conditions resulting from numerous socio-economic activities. Multiple levels of decision making are also represented in the model, starting from the highest basin-wide, ending with the individual water user. The time horizon of such studies suggests a span of a few decades at least, while it is spatially bounded to the limits of a basin. 


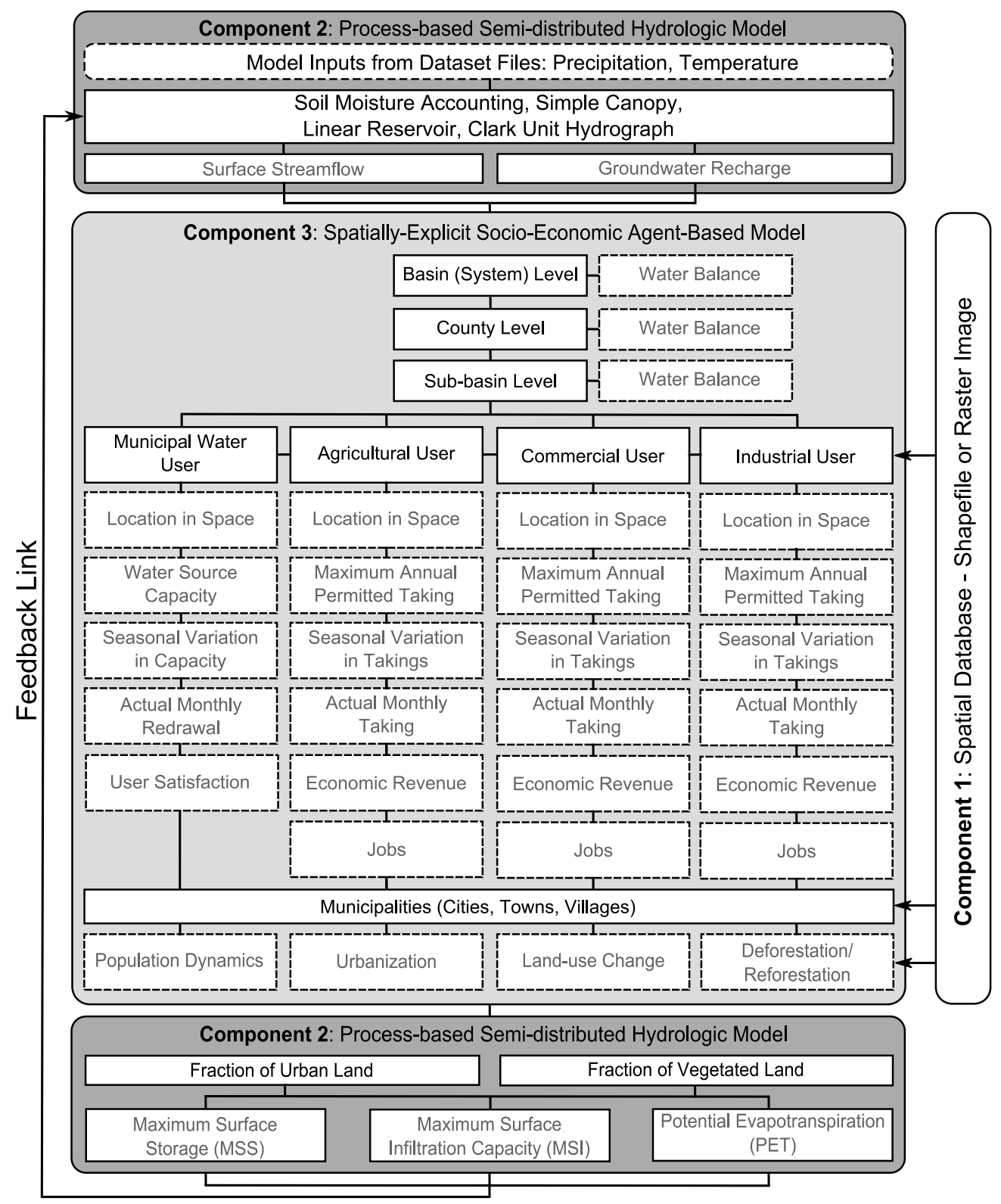

Figure 4: Detailed architecture of an operational model to support integrated water resources management

Multi-method modeling framework is designed to dynamically integrate and link all components. Theory suggests three strategies for integration of multiple modeling method components: embedded coupling, tight coupling, and loose coupling (Ahmad, 2002). Embedded coupling, also known as full integration, requires that all components are developed using common programming tools within a single data structure. 
Therefore, this coupling method does not require dedicated data transfer between the components. In tight coupling strategy, also known as integration under a common interface, different components are developed independently containing their own data structure. This approach requires a dedicated data transfer, although particular data files can be shared and used by all components. The link between different components is achieved through a common user interface. The loose coupling refers to integration of models being developed and run independently. The interaction between the components is achieved through a set of input/output files. The selection of coupling method is based on the individual complexity of developed models and functionality of multi-method modeling approach. The main requirement is dynamic data exchange (DDE) between the system components to provide a feedback under a single user interface. Therefore, the tight coupling strategy is an optimal solution.

\subsection{Level 3: Detailed description of individual components of an operational model for support of integrated water resources management}

\subsubsection{Component 1: Spatial database}

The analysis of water resources systems typically requires substantial amounts of data. Spatial or non-spatial data stored in a database model is therefore placed at the core of this modeling framework. In contrast to non-spatial database models, spatial databases are designed to store and manage information related to geometric features that are explicitly defined in geometric space. Geometric features are stored in vector form, usually as points, lines or polygons, but also can be in the form of more complex structures, such as networks or 3D objects. Theoretically, different formats are used to store spatial data related geographic locations: geodatabase, shapefile, raster image, tabular data, and coverage (ESRI, 2014).

\section{Geodatabase}

A geodatabase is a database designed to store, query, and manipulate geographic information and spatial data. This format created by ESRI is designed to utilize all features of relational database models (RDBM). It stores geographic information using a 
database management system (DBMS), and it represents a collection of geographic datasets of various types held in a particular file system folder. It is designed to create a direct access to the collection of datasets through a database management system using Structured Query Language (SQL). Many geodatabases have custom functions that allow the spatial data to be manipulated and queried using SQL. A typical geodatabase contains three primary dataset types:

- Feature Classes

- Raster Dataset

- Tables

Datasets are designed mechanisms to manage geographic information. Feature classes present tables with shape files that contain point, line, or polygon geometries for geographic features. Each row in the table presents a distinct feature. Attribute tables (such as dBase files, DBMS, Microsoft Access Tables, or Excel Spreadsheets) present a collection of rows, each containing the same fields or attributes. Finally, rater dataset is a set of imagery that describes continuous geographic phenomena. Geodatabase is the format that supports different ranges of datatypes, NULL columns, and various raster datatypes. However, due to unique requirements of tools applied in the case study, the spatial database is created in the form of shapefiles.

\section{Shapefile}

Shapefile format was created in 1990s, and it uses the dBase-III format to store attributes. A shapefile is a nontopological format that stores the geometric location and attributes information of geographic features (ESRI, 2014). In a shapefile, geographic features are

points, lines or polygons. Also, a shapefile includes dBASE tables that can store a number of additional attributes that can be related to a shapefile's features.

However, this format contains certain limitations, such as lack of numeric nulls, a tencharacter limit for column (attribute) definition, day resolution dates, and short ASCII strings. However, the most important limitation is that shapefiles use the dBASE format to store attributes. This format was developed in the 1980's, and, since then, other 
formats have improved data representation, such as Unicode, to store information in languages other than English. Also, in addition to geographic features and attributes, geographic data contain attribute relationships, topology relationships, coordinate precision and resolution, etc. However, these capabilities are not supported by the shapefile file format. Moreover, instead of binary format, shapefiles use character format to store numeric attributes. This limitation is not applied to the geographic feature coordinates, just to attributes.

The structure of shapefiles and dBASE tables can be modified by adding and deleting fields or attribute columns. One of the limitations of shapefiles is that a field's name must not contain more than 10 characters. These limitations make the shapefiles not a suitable choice for active database management, but because of their simplicity, the shapefiles are widely used open data transfer format. Therefore, the shapefiles are used in this study.

\section{Raster Images}

Spatially continuous geographic features of a particular location are typically stored as raster datasets. This format divides space into a matrix of regular squares or rectangles. Each cell in the grid holds unique information that represents a desired characteristic of that location, such as land use or land cover category, temperature, or elevation. Raster datasets originate from aerial photographs, satellite imageries or scanned maps.

Raster dataset format offers numerous advantages for storing data. Simple data structure enables a matrix of cells to represent continuous surfaces, which makes this format perfectly suitable for advanced spatial-statistics analysis. Also, this format allows the uniform storage of other geographic features, such as points, lines, polygons and surfaces.

However, raster datasets can potentially be large in terms of the storage disk size, depending on the resolution of a dataset. Generally, higher resolution of a dataset (smaller cell size) means larger data file. On the other side, lower resolution decreases the size of the file. Lower resolution, however, brings additional inaccuracies to spatial analysis due to the size cells. 


\subsubsection{Component 2: Process-based semi-distributed hydrologic model}

Hydrologic models are developed to study the basin's response to changing physical and natural conditions, such as extreme meteorological events. Process-based hydrologic models mathematically describe the physics behind complex hydrologic cycles occurring within a basin. These models analyze the movement and distribution of water through streams, rivers, ground, or aquifers, while some hydrologic models have an additional component to analyze the water quality parameters. Generally speaking, hydrologic models use precipitation and temperature as the system inputs, and transform it through a set of mathematical equations into the system output, in this case runoff.

The natural hydrologic process typically described by these models starts with the precipitation that falls on local water bodies and lands. Some quantities of water are returned to the atmosphere through evaporation and transpiration, while remaining extents of water fall through the vegetation to the land surface. Depending on the physical properties of soil and the current levels of moisture, the water may pond or it can further infiltrate to the deeper ground. Partially saturated upper surface layers temporarily store the water and then it moves upwards due to capillary action or horizontally as interflow. A portion of water then percolates to the deeper groundwater aquifers. Water from the aquifers eventually returns to the surface as baseflow. A combination of overland flow, precipitation that falls on the water body, the interflow and baseflow comprise the surface flow.

Depending on which of these physical processes are described within the model structure, hydrologic models can be divided into three groups: event-based models, continuous process models and hydrologic models capable of simulating both short-term and continuous events (Cunderlik and Simonovic, 2003). Event-based models describe a basin's response to specific precipitation events, and, therefore, they do not include dynamic processes of moisture balance between storms, long term movement of moisture, and their associated rates of evaporation and evapotranspiration. They focus on infiltration and surface runoff, while their main objective is the assessment of direct runoff. Therefore, event-based hydrologic models are primarily used to characterize and 
fully describe particular flood events, determine the spatial extents of floodplains, and to assist in design of municipal infrastructure.

In contrast to event-based models, continuous hydrologic models describe the long-term movement of moisture within a basin and evaluate the detailed soil moisture balance of dry and wet climate conditions. They include a set of methods that describe rainfall transformation, calculate baseflow, determine the propagation of a flood hydrograph through a channel or reservoir, and, finally, account for all losses through vegetation, surface, soil and groundwater. Because continuous-based models track the long-term soil moisture balance they are suitable tools for simulation of variation in daily, monthly and seasonal streamflow and estimates of seasonal water yields.

Depending how they describe spatial variability, hydrologic models can also be classified into the following three categories (Cunderlik and Simonovic, 2003):

Lumped models. Parameters used in lumped models represent the averaged values for the whole basin and often do not represent physical processes but they are obtained empirically. Spatial variability of model parameters is represented by calculating the averaged values for the whole basin. An area-weighted average is one the most often used procedures. Typically, lumped models are not applied to event-scale processes.

Semi-distributed models. By dividing the basin into a series of smaller sub-basins, these models allow spatial variation of parameters. There are two types of semi-distributed models: 1) kinematic-wave (KW) theory models and 2) probability distributed (PD) models. While the KW models represent simplified equations of the surface and/or subsurface flow of physically based hydrologic models, the probability distributed models account spatial variability by using probability distributions of input parameters across the basin.

Distributed models. In spatially distributed hydrologic models, model parameters fully vary in space depending on the resolution chosen by the user. Consequently, they require numerous data for parametrization process for each cell in a grid. Since these models 
describe governing physical processes in detail, they provide the highest degree of accuracy.

Over the years, a significant number of event-based and continuous-based hydrologic models has been developed by different institution across the world, such as Hydrological Simulation Program - Fortran (HSPF) was developed by U.S. Geological Survey (Bicknell et al. 2001), SHETRAN Hydrologic model by Newcastle University (UK) (Birkinshaw et al. 2010), Hydrologic Engineering Center - The Hydrologic Modeling System (HEC-HMS) by US Army Corp of Engineers (USACE, 2006), MIKE Système Hydrologique Europeén (MIKE-SHE) by DHI (DHI, 2004), SWMM (Rossman, 2004), Visual OTTHYMO (Greenland International Consulting Inc., 2001), and many others.

Criteria for selection of an appropriate hydrologic model depend on the specific requirements and needs of a project. However, some basic criteria must be always analyzed. In this research, the selected hydrologic model needs to represent long-term seasonal dynamics of water movement within a basin and, as a result, to provide two basic pieces of information: groundwater recharge rates and surface streamflow regimes. Also, one of the main ideas of newly designed modeling methodology is to be built on models and software packages that are free of charge and possibly open-source. This would enable the users to run, copy, distribute, study, change and improve the model according to their own needs and purposes. Finally, since the hydrologic model needs to be integrated, and thus exchange data with other models that represent the socioeconomic environment, continuous HEC-HMS model is seen as the most appropriate choice. However, due to the requirements of the tight coupling of different system components, the modular structure of a HEC-HMS model is rewritten and converted to Java programming language for easier manipulation and processing, Prodanovic (2007).

\subsubsection{Structure of the continuous hydrologic model}

HEC-HMS is a spatially semi-distributed hydrologic rainfall-runoff model, USACE (2000). Depending on the type of model being developed, event or continuous-based, a modeler chooses the optimal combination of model methods available within HEC-HMS 
- 7 infiltration methods, 6 streamflow routing methods, 3 baseflow calculation and 3 reservoir routing methods (USACE, 2006). A HEC-HMS model contains three main parts:

Meteorological module - describes precipitation input and calculation of evapotranspiration;

- Basin module - Describes physical properties of a basin;

- Simulation control module - Controls the parameters of simulation.

The structure of a typical HEC-HMS hydrologic model is separated into a number of different modules. Each module mathematically describes a particular physical process in the basin, Figure 5.

Temperature and precipitation data serve as inputs into the snow module. Based on the average daily temperature, this module adjusts precipitation, and determines if the state of precipitation is solid or liquid. Snow module produces adjusted precipitation which then is used for computation of the losses module. Losses analyzed by the model are interception, infiltration, surface storage, evaporation and transpiration. The losses module accounts the movement of moisture through a set of conceptual reservoirs within a basin, such as canopy, surface, soil and groundwater. Evapotranspiration represents one of the outputs of the loss module and includes the moisture that evaporates from the canopy, surface depressions, and soil. Second output from the loss module is baseflow that computes the lateral flow returned to the stream from ground. Also, surface excess output calculates the volume of water that remains on the surface and does not infiltrate into the soil. Finally, groundwater recharges represents flow that infiltrates deep aquifers and do not return to the surface. 


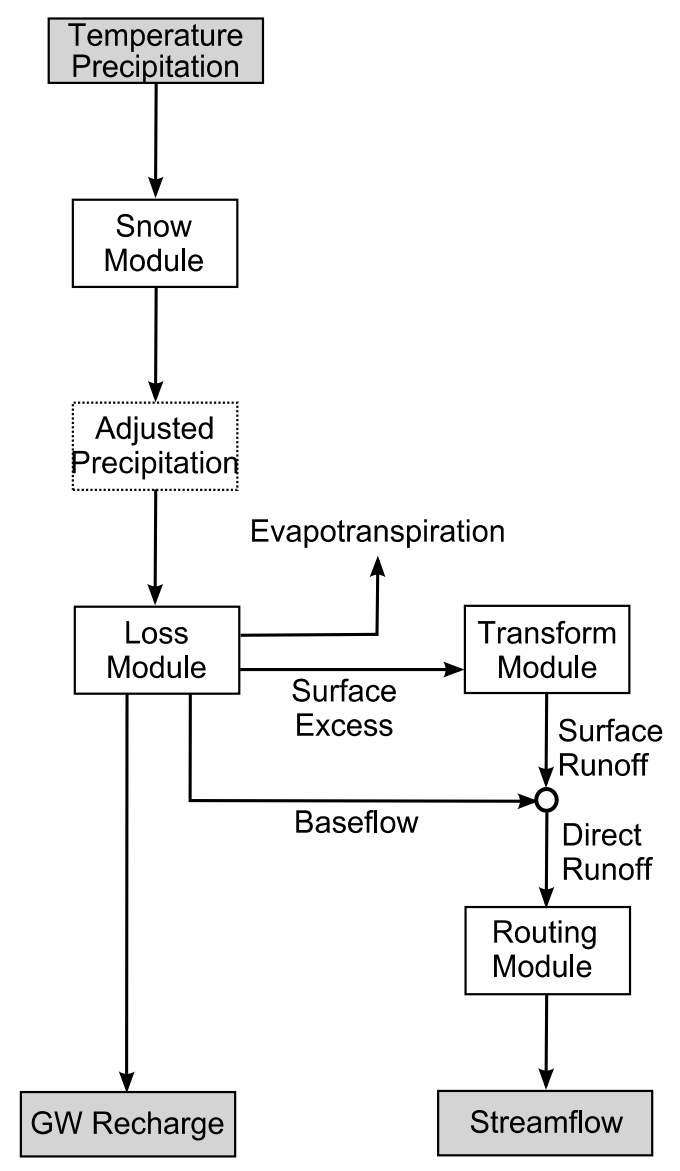

Figure 5: Modules of a hydrologic model

The surface excess is used by a transform module, where the surface excess is converted to calculate direct runoff by convoluting a unit hydrograph. Surface runoff is calculated as an output from the transform module. It is then combined with the baseflow to produce the direct runoff. Direct runoff represents an input to a routing module which calculates the propagation of a flood wave and produces the channel streamflow. Following sections give detailed mathematical formulations for each module represented in the model structure.

\subsubsection{Snow accumulation and melt module}

This module uses the Degree-Day method to calculate snow accumulation and melt rates based on the given time series of temperature and precipitation (Cunderlik and Simonovic, 2004). Since the climatic data is available for certain stations and their specific locations within the basin, spatially interpolated values of precipitation and 
temperature for each sub-basin are calculated using the Inverse Distance Weighting Method (USACE, 2000). Interpolated data is then separated into solid and liquid forms of precipitation, Figure 6. The solid form is used by an accumulation and melt algorithm to calculate the snowmelt rates. After that, the snowmelt is combined with the liquid form of precipitation to produce adjusted precipitation. This adjusted precipitation is an input to the loss and other modules.

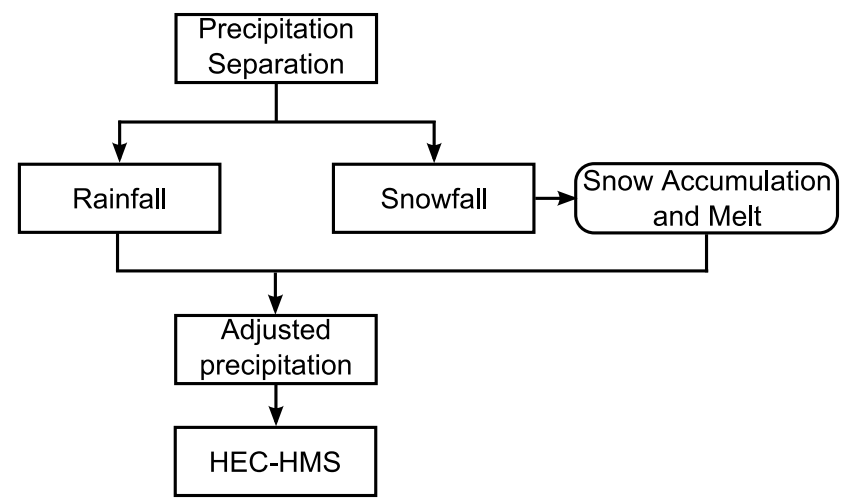

Figure 6: Snow module algorithm

The algorithm of snow module begins after the interpolation of temperature and precipitation values. If the average daily temperature $T_{t}$ is less than the minimum temperature for snowfall $T-=-4{ }^{\circ} C$, the precipitation takes solid form:

$$
\begin{aligned}
S_{t} & =P_{t} \\
R_{t} & =0.0
\end{aligned}
$$

In this case, $P t$ is the observed amount of precipitation in [mm/day], while $S_{t}$ and $R_{t}$ represent volumes of precipitation that fall as snow or rain. Index $t$ represents the simulation time step for the total number of days for which precipitation data is available. If the average daily temperature takes a range between the minimum $T_{-}=-4{ }^{\circ} \mathrm{C}$ and maximum $T_{+}=-2{ }^{\circ} \mathrm{C}$, the snowfall and rainfall are calculated according to:

$$
\begin{gathered}
S_{t}=P_{t} *\left[\frac{T_{+}-T_{t}}{T_{+}-T_{-}}\right] \\
R_{t}=P_{t}-S_{t}
\end{gathered}
$$

Finally, if the average daily temperature is greater than the maximum temperature: 


$$
\begin{gathered}
S_{t}=0.0 \\
R_{t}=P_{t}
\end{gathered}
$$

The solid form of precipitation is used by an accumulation and melt algorithm to compute the snowmelt. The daily volume of melt is computed by:

$$
M_{t}=M R *\left(T_{t}-T_{c r}\right)
$$

where $M R$ represents melt rate $\left[\mathrm{mm} /{ }^{\circ} \mathrm{C} / \mathrm{day}\right]$ and takes value of $4 . T_{c r}$ is a critical melt temperature set to zero. Calculated snowmelt is accumulated by following equation:

$$
S_{t}=S_{t}+S_{t-1}
$$

This value is used to calculate the adjusted precipitation. If snowmelt occurs $\left(M_{t}>0\right)$ and the accumulated snowmelt $S_{t}$ is greater than the melt rate $M_{t}\left(S_{t}>M_{t}\right)$, only a portion of the accumulated snow melts:

$$
\begin{aligned}
& S_{t}=S_{t}-M_{t} \\
& P_{a}=R_{t}-M_{t}
\end{aligned}
$$

here, $P_{a}$ represents the adjusted precipitation in [ $\left.\mathrm{mm} / \mathrm{day}\right]$.

On the other hand, if all accumulated snow melts, the adjusted precipitation is:

$$
P_{a}=R_{t}+M_{t}
$$

Finally, if no snowmelt occurs, the adjusted precipitation is:

$$
P_{a}=R_{t}
$$

\subsubsection{Soil moisture accounting loss module}

The losses module represents the most complex component of the hydrologic model. This module uses a series of conceptual reservoirs to represent the quantities of stored water and describes the water movement in each sub-basin. This module computes runoff volume by subtracting from the adjusted precipitation volumes of water that is intercepted, infiltrated, stored, evaporated and transpired. The storage reservoirs 
represent: a) canopy interception; b) surface interception; c) soil profile; d) a number of ground water layers.

The soil moisture accounting module, Figure 7, represents a sub-basin as a series of storage layers. Storage content is computed for each time step and it varies continuously during and between the storm events (USACE, 2006) For given rates of precipitation and potential evapotranspiration (ET), this algorithm calculates basin surface runoff, losses due to evapotranspiration, groundwater flow, and deep percolation over the entire basin.

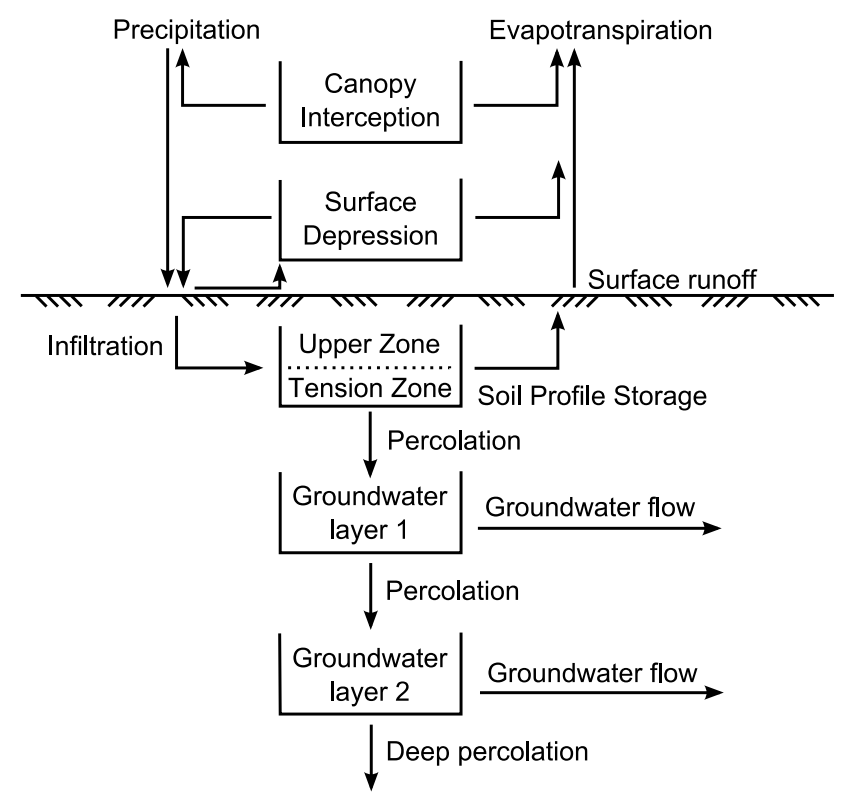

Figure 7: Soil moisture accounting algorithm

\subsection{Canopy-interception and surface depressions}

Interception and surface storage layers estimate the quantities of water stored by vegetation (trees, bushes, grass, plants, etc.), ground surface depressions, and any other surface areas where water is not free to move as overland flow. If the layer is not already at full capacity, adjusted precipitation is the single inflow to the canopy-interception storage layer. On the other hand, the single process that takes water volume from the canopy-interception layer is the process of evapotranspiration. When this storage layer is filled with water, then the precipitation is available to fill the surface-interception storage layer. This layer describes local shallow depressions and cracks on the ground surface. If the soil is not saturated, this amount of water is available for infiltration. When the 
surface storage layer is filled, then the surface runoff is produced by the amount of water that cannot be absorbed by the soil.

\subsection{The soil profile storage}

The soil-profile storage represents the top layer of the soil. Inflow to this layer is water that infiltrates from the surface, while percolation to deeper groundwater layers and evapotranspiration are the outflows from the layer. This layer contains two zones: the upper zone and the tension zone. The upper zone loses water to both percolation and evapotranspiration because it represents the water held in the pores, while the tension zone losses water to evapotranspiration since it only contains water detained by capillary tension.

\subsection{Evapotranspiration}

Evapotranspiration extracts water from the canopy, surface and soil storage layers. Process of evapotranspiration occurs only in periods with no precipitation. Potential evapotranspiration (PET) parameter depends on the maximum regional monthly rate of evapotranspiration multiplied by the pan coefficient. The rate of actual evapotranspiration depends on the loss of moisture from the canopy, surface, and soil storage. The water percolates from the soil profile storage to the groundwater layer. The volume of water returned to the stream channels as baseflow and the volume of water that percolates deeper underground represent two outflows from this layer.

\subsection{Soil moisture accounting losses module}

Because of their complexity, the detailed mathematical equations of this module can be found in the literature, and, therefore, only key mathematical relationships are shown here. A set of differential equations are used to describe dynamics of canopy A, surface $\mathrm{B}$, soil C, top D and bottom E ground water layers, all in [mm]:

$$
\begin{gathered}
d A / d t=P_{t}-E T_{t}^{A} \\
d B / d t=P_{t}^{B}-E T_{t}^{B}-S_{t}^{E}
\end{gathered}
$$




$$
\begin{gathered}
d C /_{d t}=I_{t}-R_{t}^{C}-E T_{t}^{C} \\
d D /_{d t}=R_{t}^{C}-G W F_{t}^{D}-R_{t}^{D} \\
d E /_{d t}=R_{t}^{D}-G W F_{t}^{E}-R_{t}^{E}
\end{gathered}
$$

where $P_{t}$ is precipitation, $E T_{t}$ evapotranspiration (from canopy $E T_{t}^{A}$, surface $E T_{t}^{B}$, and soil storage layers $\left.E T_{t}^{C}\right), P_{t}^{B}$ precipitation after canopy, $S_{t}^{E}$ surface excess, $I_{t}$ infiltration, $R_{t}$ percolation (from/to soil $R_{t}^{C}$, ground water $1 R_{t}^{D}$ and $2 R_{t}^{E}$ ), and $G W F_{t}$ lateral ground water flow (from layers $1 G W F_{t}^{D}$ and $2 G W F_{t}^{E}$ ), all in [mm/hr].

Soil infiltration is calculated after computing the potential soil infiltration, $I_{t}^{P}[\mathrm{~mm} / \mathrm{hr}]$ :

$$
I_{t}^{P}=I_{m}-\left(C_{t} / C_{m}\right) I_{m}
$$

where $I_{m}$ is the maximum soil infiltration $[\mathrm{mm} / \mathrm{hr}], C_{t}$ is the volume of water in soil [mm], and $C_{m}$ is the maximum volume in soil storage [mm]. If the soil contains little or no water, the potential infiltration can be as high as the infiltration capacity. If the soil is saturated, the potential infiltration will be small. The actual soil infiltration $I_{t}$ at time $t$ is calculated as the minimum value between the water availability for infiltration $A W_{t}$ and the potential soil infiltration $I_{t}^{p}$ :

$$
I_{t}=\min \left(A W_{t}, I_{t}^{p}\right)
$$

Percolation is the water transferred from the soil storage to a ground water layer. The potential soil percolation $R_{t}^{C, p}$ is computed:

$$
R_{t}^{C, p}=R_{m}^{C}\left(C_{t} /_{m}\right)\left(1-D_{t} /_{D_{m}}\right)
$$

where $R_{m}^{C}$ represents the maximum soil percolation in $[\mathrm{mm} / \mathrm{hr}], C_{t}$ is the volume of water in soil storage $[\mathrm{mm}], C_{m}$ is the soil storage capacity in $[\mathrm{mm}], D_{t}$ represents the current ground water layer storage, and $D_{m}$ stands for the maximum ground water storage in $[\mathrm{mm}]$. Actual infiltration represents the minimum value between the potential soil 
percolation and the amount of water available for percolation. The groundwater outflows are lateral ground water flows. Lateral flow in the SMA algorithm is calculated:

$$
G W F_{t}=\frac{\left(R_{t}^{C} \Delta t\right)+G W S_{t-1}-\left(P G W P_{t} \Delta t\right)-\left(0.5 G W F_{t-1} \Delta t\right)}{k+0.5 \Delta t}
$$

where $G W F_{t}$ is the ground water $[\mathrm{mm} / \mathrm{hr}] ; P G W P_{t}$ potential ground water percolation $[\mathrm{mm} / \mathrm{hr}]$; and $k$ is ground water storage coefficient [hr]. Lateral flow is averaged, before being routed with a series of linear reservoirs to produce baseflow.

\subsubsection{Transform module}

Calculated surface water excess obtained from the soil moisture accounting (SMA) module is converted into direct runoff using Clark's unit hydrograph method. A graphic representation of this method is shown in Figure 8.

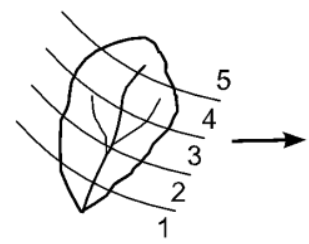

a. Time Area Map of Basin

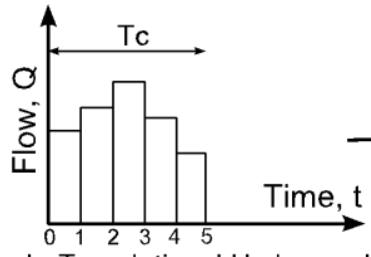

b. Translational Hydrograph

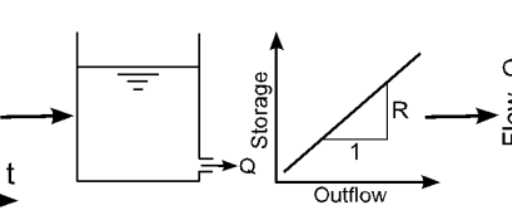

c. Linear Reservoir Routing

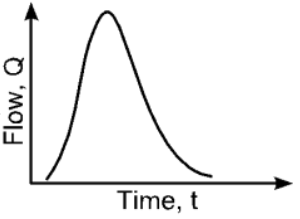

d. Instantaneous Unit Hydrograph

Figure 8: Clark's unit hydrograph method

The first step in this method is selection of a time-area relationship (Figure 8a) given by:

$$
\begin{gathered}
A_{I}=1.414\left(t / T_{c}\right)^{3 / 2} 0 \leq t / T_{C} \leq 0.5 \\
1-A_{I}=1.414\left(1-t / T_{c}\right)^{3 / 2} 0.5 \leq t / T_{C} \leq 1
\end{gathered}
$$

In these equations, $A_{I}$ is the cumulative fraction of a basin area and $t / T_{C}$ is a fraction of the time of concentration. This assumption uniformly distributes the velocity of overland flow over the basin. Also, the time needed for runoff to reach the basin outlet is proportional to the travelled distance. Therefore, this dependence represents a temporal distribution of excess rainfall on the surface. 
A translational unit hydrograph (Figure 8b) is used to convert surface excess to direct runoff. This hydrograph is constructed on the basis of the assumed time-area relationship, catchment size, and time of concentration $T_{c}$. If no attenuation takes place, the translational unit hydrograph defines the flow through the basin outlet. Calculation of transitional hydrograph requires time-area relationship and the total basin area. The volume of the translational hydrograph is equal to the uniform rainfall of one unit falling over the basin for the duration equivalent to the time of concentration.

In case of hydrograph attenuation, the transitional hydrograph is routed through a linear reservoir, Figure 8c. The slope of the storage-outflow function $(R)$ is then defined since it is needed to obtain an instantaneous unit hydrograph. The linear reservoir routing uses a discrete approximation of the continuity equation combined with a linear storage outflow function:

$$
I_{t}-\frac{O_{t-1}+O_{t}}{2}=\frac{S_{t}-S_{t-1}}{\Delta t}
$$

In this case, $I_{t}$ represents an average inflow at time $t$ (ordinate of the translational hydrograph), while $O_{t}$ and $S_{t}$ are the outflow and storage during $\Delta t$. Storage in the linear reservoir is then approximated:

$$
S_{t}=R O_{t}
$$

by substituting two previous equations:

$$
I_{t}-\frac{O_{t-1}-O_{t}}{2}=\frac{R O_{t}-R O_{t-1}}{\Delta t}
$$

and, finally, substituting:

$$
c=\frac{2 \Delta t}{2 R+\Delta t}
$$

In equation (27) results in:

$$
O_{t}=c I_{t}+(1-c) O_{t-1}
$$


The unit hydrograph is calculated by averaging two instantaneous unit hydrographs:

$$
U_{t}=\frac{O_{t}+O_{t-1}}{2}
$$

In this equation $U_{t}$ represents the ordinate of the unit hydrograph. To get direct runoff of the catchment $Q_{d}^{t}$, the unit hydrograph is transformed using the convolution equation in discrete form:

$$
Q_{t}^{d}=\sum_{i=1}^{t} E_{i} U_{t-i+1}
$$

where $E_{i}$ is the rainfall excess.

\subsubsection{Reservoir and river routing}

Surface water movement is described using a hydrologic routing method, the Modified Puls Method. The reservoir and river routing calculate the propagation of a flood wave as it passes through a series of river reaches and reservoirs. The computational procedure is similar to the linear reservoir method used in transform module. The only difference is that nonlinear storage-outflow functions are applied. This particular method uses a hydrograph as input (Figure 9a), passes it through a nonlinear reservoir (Figure 9b), and finally produces a modified flood hydrograph as output (Figure 9c).

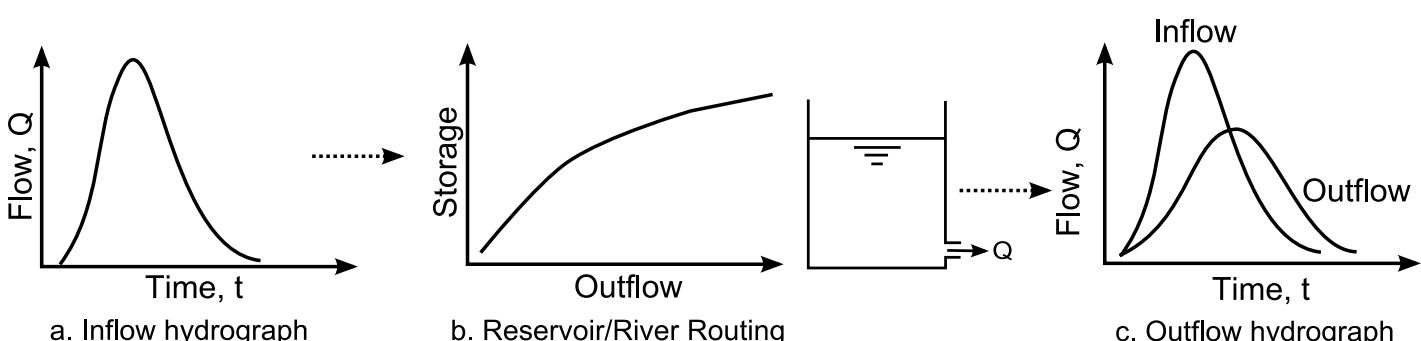

Figure 9: Modified puls routing method

\subsubsection{Component 3: Spatially explicit socio-economic agent-based model}

Agent-based modeling method is used to represent the spatially explicit socio-economic component of water resources systems. Railsback and Grimm (2011) define this 
modeling method as one in which individual system entities, also referred to as agents, are described as unique and autonomous units that interact with each other and their local environment. This modeling approach recognizes the individual-centric behavior as the central driver of complex system's behavior. In agent-based models, the global system behavior emerges as a result of interactions between agents, how they perceive and respond to each other, and interactions between agents and their environment. In other words, the macro-level system behavior in agent-based models is a result of the microlevel behavior of the agents. For that reason the agent-based modeling method is also known as the disaggregated individual-centric (bottom-up) simulation method (Grimm and Railsback, 2005). Depending on the scale and purpose of the system being represented by the model, agents may take numerous forms (for example, people, infrastructural elements, companies, banks, vehicles, cities, farmers, or animals) as long as they have their own individual properties and pursue certain goals as individuals or as members of a group. Uniqueness or individuality of entities implies that agents have unique features, such as gender, age, size, location, or resource reserves. Individual behavior is described for each agent and it is governed by their individual structure and properties, such as memory, reaction, sensitivity or current state. On the other side, being autonomous means that agents can act independently and pursue their own set of objectives (Railsback and Grimm, 2011). This implies that agents adapt their behavior to their own current state, state of other agents, and state of their environment. In these models, each agent lives in a certain environment and can be connected to other agents. Interaction between agents is mainly of local character since they do not interact with all other agents, but only with their neighbors in a geo-spatial or social sense (for example, through social networks). The environment described in agent-based models can be physical, geographical, social, or information space.

\subsubsection{Features of agents}

Although agents represent the core of agent-based models, researchers have not come to a full agreement on the acceptable definition of an agent. Wooldridge (2009) suggests a definition that defines an agent as an autonomous entity that is situated in some 
environment, is able to perceive it through physical sensors or data files, and is capable of autonomous action in this environment in order to meet prescribed objectives, Figure 10.

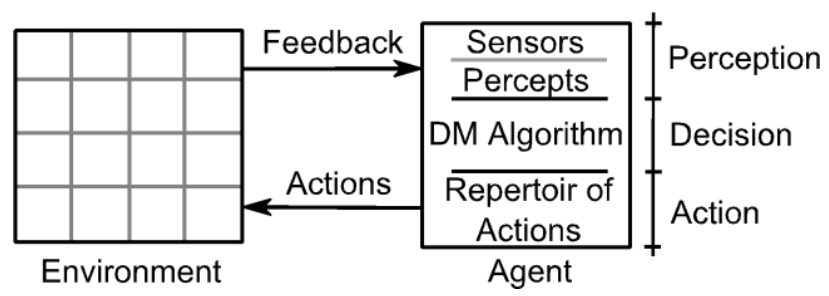

Figure 10: An agent's architecture

All actions taken by an agent are directed toward achieving desired and prescribed objectives. Additionally, Wooldridge (2009) recognizes a number of unique features that characterize agents, such as autonomy, heterogeneity, and activity. Agents are considered to be autonomous entities if they can process information, exchange it with other agents and act autonomously upon it. Moreover, heterogeneity means that all individual entities have different and unique attributes that drive their behavior. Finally, activity means that each individual agent exercises individual influence in a simulation. In addition, Schieritz and Milling (2003) identify a set of active features that can characterize an agent, see Table 2.

Table 2: Typical properties of an agent

\begin{tabular}{|c|c|}
\hline Properties & Description \\
\hline $\begin{array}{l}\text { Pro-active/goal directed } \\
\text { behavior }\end{array}$ & $\begin{array}{l}\text { Agent has prescribed objectives to achieve with respect } \\
\text { to its behavior }\end{array}$ \\
\hline Situatedness & $\begin{array}{l}\text { Agent is embedded in its environment, and is able to } \\
\text { perceive it and act on it }\end{array}$ \\
\hline Reactiveness/Responsiveness & $\begin{array}{l}\text { Agent is able to timely react to all changes in the } \\
\text { environment }\end{array}$ \\
\hline Autonomy & Agent is able to control its own actions and internal state \\
\hline Social Ability & $\begin{array}{l}\text { Agent is able to interact and communicate with other } \\
\text { agents }\end{array}$ \\
\hline Antropomorphity & Agent has human-like attributes, beliefs and intentions \\
\hline Learning & $\begin{array}{l}\text { Agent is able to perform better over time based on } \\
\text { previous experience }\end{array}$ \\
\hline Continuity & Temporally continuous running process \\
\hline Mobility & $\begin{array}{l}\text { Agent is able to move around the simulated physical } \\
\text { space }\end{array}$ \\
\hline Specific Purpose & Agent is designed to achieve well-defined tasks \\
\hline
\end{tabular}


Agent-based models typically include more than one agent and they represent a system of agents, Figure 11. According to Schieritz and Milling (2003), such systems have four main characteristics:

- Each agent does not have complete information and capabilities to solve a given problem;

- System does not contain the global system control;

- Data is decentralized; and

- Computation is asynchronous.

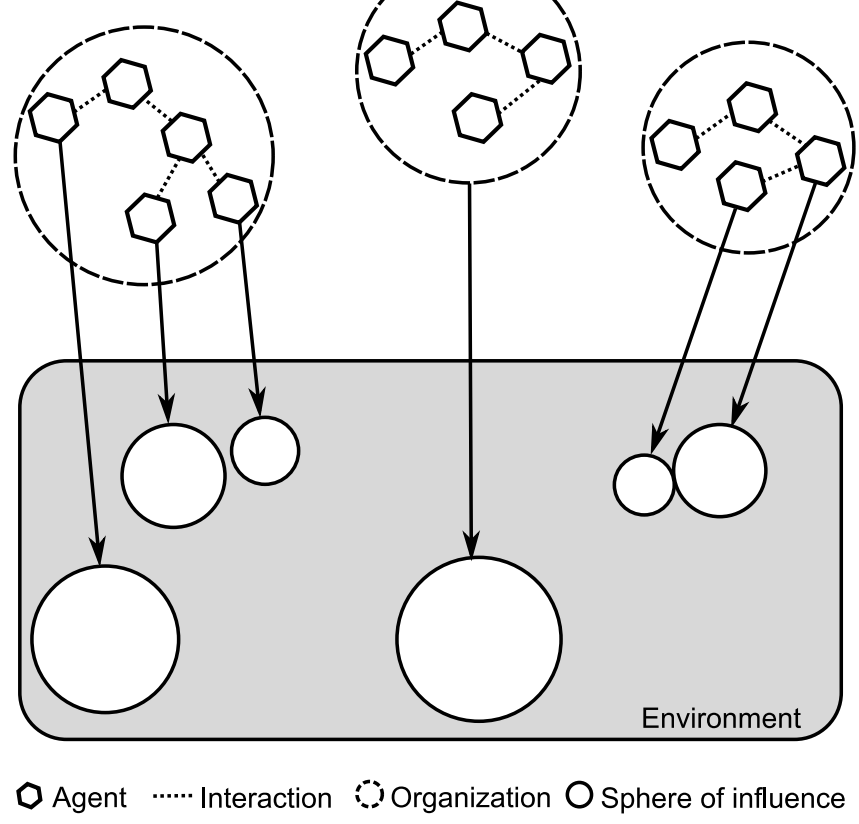

Figure 11: Typical structure of a system of agents (source Wooldridge, 2009)

In such a way, agent-based models disaggregate systems into a set of individual components that can have their own characteristics. Consequently, the most important property of individual agents is a set of rules that drives their behavior and the type of interaction with other agents and the environment. In some instances, all agents in the system can have identical set of rules that influence their behavior. However, due to their external and internal heterogeneity, agents typically have diverse set of rules. Set of rules 
that drives their behavior can be defined by advanced computational algorithms. Depending on their structure, agents can be driven by simple rules (for example, a thermostat can be observed as a purely reactive agent, which has two defined actions - on or off, depending on the perception of temperature) or very complex - described by complex behavioral models in domain of cognitive science and artificial intelligence (North and Macal, 2009). In the domain of social sciences, recently there has been a move towards incorporating behavioral frameworks within agent-based models to better represent human behavior and Kennedy (2012) provides an overview of different frameworks for describing the human behavior in $\mathrm{AB}$ models.

\subsubsection{Abstract architecture of intelligent agents}

According to Schieritz and Milling (2003), there is still no universally accepted mathematical formalism for agent-based models. However, Wooldridge (2009) offers a way to mathematically define the formal abstract architecture of agents. Wooldridge (2009) assumes that the environment can be described as a finite set of discrete and instantaneous states:

$$
E=\left\{e, e^{\prime}, e^{\prime \prime}, \ldots\right\}
$$

This assumption applies even in the case of continuous environments since they can be represented as a finite set of discrete environments with a desired degree of accuracy. At the same time, agents are assigned a number of actions that transform the environment:

$$
A_{C}=\left\{\alpha, \alpha^{\prime}, \alpha^{\prime \prime}, \ldots\right\}
$$

The typical model of interaction between agents and the environment starts with the current state of the environment. Based on that, the agent chooses an action to perform. Consequently, the environment reacts to a particular action and responds with a number of possible states. However, only one possible state actually occurs. Based on this second state the agent again performs an action from the repertoire of available actions. A set of agent's actions and respective states of the environment are referred to as runs:

$$
r: e_{0} \stackrel{\alpha_{0}}{\rightarrow} e_{1} \stackrel{\alpha_{1}}{\rightarrow} e_{2} \stackrel{\alpha_{2}}{\rightarrow} \ldots \stackrel{\alpha_{u-1}}{\longrightarrow} e_{u}
$$


where:

$R$ is the set of all possible finite sequences $\left(E\right.$ and $\left.A_{c}\right)$;

$R^{A c}$ is the subset of these that end up with an action;

$R^{E}$ is the subset of these that end up with an environment.

Then a state transformer function is used to represent the effect that agent's actions have on the environment:

$$
\tau: R^{A c} \rightarrow 2^{E}
$$

Environments are assumed to be history dependent. The next state of an environment is determined by the agent's actions, the current state of environment and the actions taken earlier by the agent. Formally, the environment is defined as a triplet $E n v=\left(E, e_{0}, \tau\right)$, where $E$ is the set of environment states, $e_{0}$ is an initial state, while $\tau$ is a state transformer function.

Purely reactive agents

However, some types of agents decide what to do without reference to their history. Purely reactive agents respond directly to their environment. The behavior of a purely reactive agent is defined as a function:

$$
A g: E \rightarrow A C
$$

Since the agent is created with particular reason to perform tasks in the simulation, it is required to properly communicate the desired tasks to the agent. The advantage of intelligent agents is that they can be instructed what to do, without instructing them how to do it (Wooldridge, 2009). To ensure this, some kind of performance measure should be used. There are several ways to define a performance measure. The first one is through the association of utilities with states of environment. 


\section{Utility function}

Utility is defined as a numeric value that is used for assessing how 'good' an environment state is: higher the utility value, the better the state. Therefore, a goal can be given to an agent to achieve states that maximize the utility without specifying how this is to be done. A task specification is a simple function:

$$
u: E \rightarrow R
$$

\section{Maximization of the expected utility function}

If it is assumed that the utility function $u$ has an upper bound, an optimal agent can be identified. The optimal agent is one that maximizes expected utility. If $P(r \mid A g, E n v)$ is assumed to denote the probability that run $r$ occurs when the agent Ag is placed in environment Env:

$$
\sum_{r \in R(A g, E n v)} P(r \mid A g, E n v)=1
$$

then, the optimal agent $A g_{\text {opt }}$ in an environment is one that maximizes expected utility:

$$
A g_{\text {opt }}=\arg \max _{A g \in A G} \sum_{r \in R(A g, E n v)} u(r) P(r \mid A g, E n v)
$$

\section{Interactions between agents}

One of the major advantages of the agent-based modeling method lies in agent's ability to interact with other agents towards achieving a given goal. This interaction is also known as the "social ability" of intelligent agents (Wooldridge, 2009). There are different types of interactions that can take place between self-interested agents in order to make the best decision about what action to perform. Interactions typically end in reaching an agreement between agents. Generally, there are different types of agreements: 
1. A group of self-interested agents selects an outcome from a range of possibilities based on social theory or voting;

2. Reaching decisions by binding agreements between the agents, which potentially can lead to forming coalitions;

3. Decisions based on problem of allocation of scarce resources through auctions where agents value these resources differently;

4. Agreement reached by bargaining and negotiating, and, finally,

5. How to reach agreement after conflicts of belief through argumentation.

However, detailed social interactions between multiple agents and corresponding mathematical formalisms are beyond the scope of this research. For more details on these topics the reader is referred to Wooldridge (2009) and Vidal (2010).

Agent-based modeling of water resources systems

Since agent-based modeling methods represent systems where individual entities are described as autonomous units that interact with each other and the local environment, from the integrated water resources management perspective, this approach offers a way to model all aspects of complexity of water resources systems.

In case of water resources systems, depending on the purpose of the model, agents can take numerous forms. For example, agents can represent individual ground or surface water users, water polluters, different infrastructural elements (such as dams, water treatment plants, etc.), cities and municipalities, or decision and policy makers on different levels. Each agent can be characterized by their own set of behaviors. Individuality of agents can be exploited to describe their unique features, such as individual water demand, seasonal variation of water demand, spatial location, economic activities, water availability or resilience to water scarcity.

One of the most important aspects of agents' behavior is their interaction with other system entities and the environment. Therefore, agents can be given the "social skills" to study different types of interaction between agents (coalitions forming, allocating scarce resources, negotiations, or argumentation) and how different types of interactions 
influence the system performance. Depending on the objectives given to agents and their social abilities, agent-based models can represent the complex system objectives and opposing interests of different entities. All these features can be utilized to represent the social and environmental aspects of water resources systems.

Also, agent-based models facilitate analysis of emergence within water resources systems. As agent-based simulation focuses on modeling the behavior of the heterogeneous entities and their interaction, this modeling approach is seen as bottom-up modeling approach. Bottom-up processes are, on the other hand, seen as the source of phenomena called emergence. According to Schieritz and Milling (2003), emergence occurs when interactions among objects at one level give rise to different type of objects at another level. A phenomenon is emergent if it requires new categories to describe it that are not required to describe the behavior of the underlying components

\subsubsection{Space in agent-based models}

The agents continuously interact with the local environment throughout a simulation. The key advantage the agent-based models have to offer is their ability to explicitly describe the complex interdependencies and feedbacks between the two system components agents as system actors and their environment. The environment can be described as the geographical or social space surrounding agents in whom they function. Despite the fact that agents can be static without ability to change location, agents within an environment can be spatially explicit, meaning they have a particular location in geometrical space as an attribute (Railsback and Grimm, 2011). Spatially explicit models observe space as an integral part of the system and they contain explicit links between the two. On the other hand, agents within an environment can also be spatially implicit, meaning that their location within the environment is irrelevant. In spatially explicit models, the agent's behavior fundamentally depends on both its location and the state of environment since they can alter the agent's features. Depending on the model context, this may involve the agent utilizing resources at its current location and altering the state of the location (for example - land use change models) or simply updating its current map of the environment

(for example, transportation models). In spatially explicit models, the purpose of a particular model defines the level of details used to describe the environment. In order to 
represent the environment spatially explicit models divide space into grids - a set of regular cells. This concept resembles the one used by the Cellular Automata (CA) modeling method (Heppenstall, 2012). For both modeling methods, substantial applications of remotely sensed data in raster format, functionality of GIS for processing raster-based data, and computational efficiency of regular grids have favored a partitioning space into a series of regular cells. However, in addition to CA's ability to describe spatial phenomena in terms of interactions between cells, AB models support an implementation of an actor-based processes and the dynamic change in interactions between agents and the environment. To justify the use of spatially explicit AB models, it is important that each agent has a different relationship with the environment, even in most simple terms like a location in the environment. In spatially explicit models, agents are able to evaluate the spatial configuration of environments. This ability may be as simple as determining if the availability of some resource at the current location is sufficient for some purpose or it is greater at neighboring locations.

Agent-based models and spatial variability of water resources systems

Explicit definition of space is the most important advantage of an agent-based modeling method and can be effectively used to describe the spatial variability of water resources systems. The environment can be described as the geographical space in which agents function, such as watersheds. Since spatially explicit models study space as an integral part of the systems, the agent's behavior fundamentally depends on its location and the state of environment.

\subsubsection{Overview, Design Concepts, and Details (ODD) Protocol}

The spatially explicit agent-based modeling method is used to simulate the utilization of common natural resources by numerous users. Not only that this model analyses the effects of availability of local resources on recognized stakeholders, it also simulates the effects that actions of stakeholders have on the environment, in this case hydrologic regimes. In this model the common resources are ground and surface water and land, used by different economic, agricultural, social and infrastructural entities in the system. This disaggregated agent-based model simulates the dependencies between the most 
dominant socio-economic entities and the local natural resources by assuming the strong relationship between the individual water demand and economic activities on both individual and system level. Depending on the actions of the represented stakeholders and applied management policies in the system, the model results may reveal a phenomenon called "Tragedy of the Commons". This is a phenomenon where a common good or resource is over-utilized in time due to lack of appropriate managerial practices, leaving the significant effects on both socio-economic and natural environment.

However, due to the complexities of the system being represented, the detailed description of the agent-based model must be carefully structured. Each modeling method contains a set of unique rules and conventions that help us formulate and design models. Traditional modeling methods applied in water resources management mainly use differential equations, ordinary or partial, as the most important conceptual framework. System dynamics simulation also relies on differential equations. Yet agent-based models are structurally more complex, and this is the main reason that traditional conventions are not suitable in this case.

Based on the experience of modelers, Railsback and Grimm (2011) suggest a standard protocol for describing agent-based models. Overview, design concepts, and details $(O D D)$ is a protocol designed to facilitate factual model description and organize relevant information in a consistent manner. ODD provides a way to think about and describe the agent-based modeling problems. First three elements of ODD protocol give a general overview of the model and how it is designed, Table 3. They are followed by a set of 11 design concepts that present the detailed characteristics of the models. At the end, three elements are used to give additional details and complete the description.

\section{i. Purpose}

First element in ODD protocol gives information about the main question that is addressed by the model. Also, this element gives additional details related to the system being modeled, and also suggests the question about what are we trying to learn from it? 
Table 3: Elements of the ODD Protocol

\begin{tabular}{|c|c|c|}
\hline Overview & $\begin{array}{l}\text { i. } \\
\text { ii. } \\
\text { iii. }\end{array}$ & $\begin{array}{l}\text { Purpose } \\
\text { Entities, state variables, and scales } \\
\text { Process overview and scheduling }\end{array}$ \\
\hline $\begin{array}{c}\text { Design } \\
\text { Concepts }\end{array}$ & iv. & $\begin{array}{ll}\text { Design concepts } \\
. & \text { Basic principles } \\
. & \text { Emergence } \\
. & \text { Adaptation } \\
. & \text { Objectives } \\
. & \text { Learning } \\
. & \text { Prediction } \\
. & \text { Sensing } \\
. & \text { Interaction } \\
. & \text { Stochasticity } \\
. & \text { Collectives } \\
. & \text { Observation } \\
\end{array}$ \\
\hline Details & $\begin{array}{l}\text { v. } \\
\text { vi. } \\
\text { vii. }\end{array}$ & $\begin{array}{l}\text { Initialization } \\
\text { Input data } \\
\text { Sub-models }\end{array}$ \\
\hline
\end{tabular}

ii. Entities, state variables and scales

Second element recognizes what system entities are represented in the model. Typically, agent-based models represent a set of agents (one type or more), the physical or social environment where they live and interact, and, finally, the global environment (context) that can affect all agents. Also, this element lists all variables that characterize different groups of entities. Model entities are characterized by their state variables and their state depends on their internal properties or attributes (age, sex, demand, etc.). It also depends on their behavioral strategies, such as bidding strategy, learning algorithms, etc.

Spatial scale defines the spatial extent of the model. Spatially explicit models use the spatial location in the environment as an attribute of agents. The environment is typically represented discretely by square cells, but it also can be continuous, meaning that each point has a distinct set of environmental variables. In contrast to Cellular Automata, each cell can contain one to many state variables, and they can be dependent on the global variables. Global variables are model variables that change in time, but not necessarily in space. 
Temporal scales describe the representation of time in the model. Time in AB models is usually represented using discrete time steps, such as days, months, or years. The temporal extent of an $\mathrm{AB}$ model defines the length of a simulation. Temporal resolution depends on key attributes and behaviors represented by the agents.

\section{iii. Process overview and scheduling}

This ODD element describes the model dynamics and processes that alter attributes of model entities. Scheduling element defines the order of execution of processes and provides a step-by-step outline of the whole model. In this element, a sequence of actions is defined, where an action specifies what entity executes which process in what order.

iv. Design concepts

Design concepts describe all important characteristics of a model that cannot be prescribed using other conceptual modeling methods, such as differential equations. Table 4 shows the list of design concepts and key questions.

\section{Initialization}

This element defines the initial conditions (individual attributes and global variables) of the model at the beginning of simulation.

\section{vi. Input Data}

Some models need external variables that change over time and are read from other data files as the model executes.

\section{vii. Sub-models}

Sub-models describe the core of agent-based models since they describe all major processes. The sub-models are listed in the order of execution and they must be described in details, including all equations, logical rules, or algorithms that constitute the model. 
Table 4: Design concepts key questions

\begin{tabular}{|c|c|}
\hline Concept & Key question \\
\hline $\begin{array}{c}\text { Basic } \\
\text { Principles }\end{array}$ & What general concepts and hypothesis underlie the model's design? \\
\hline Emergence & $\begin{array}{l}\text { Which of model's important results emerge from mechanistic } \\
\text { representation of the adaptive behaviors of individuals, and which are } \\
\text { imposed by rules that force the model to produce certain results? }\end{array}$ \\
\hline Adaptation & $\begin{array}{l}\text { What adaptive behavior do agents have? What decision do agents make } \\
\text { to respond to changes in their environment? }\end{array}$ \\
\hline Objectives & $\begin{array}{l}\text { What measures agents to assess their decision alternatives? How does the } \\
\text { objective measure represent processes that link adaptive behaviors to } \\
\text { important variables of the agents and their environment? }\end{array}$ \\
\hline Learning & $\begin{array}{l}\text { Do agents change their adaptive traits over time as a consequence of their } \\
\text { experience? }\end{array}$ \\
\hline Prediction & How do agents predict future conditions in their adaptive traits? \\
\hline Sensing & $\begin{array}{l}\text { What variables of their environment and themselves are agents assumed } \\
\text { to sense and therefore be able to consider in their behavior? }\end{array}$ \\
\hline Interaction & $\begin{array}{l}\text { How do agents interact? With each other agents does an agent interact? } \\
\text { At what spatial and temporal scales they occur? }\end{array}$ \\
\hline Stochasticity & $\begin{array}{l}\text { How are stochastic processes used in the model? Are stochastic processes } \\
\text { used to initialize the model? }\end{array}$ \\
\hline Collectives & $\begin{array}{l}\text { Are collectives (aggregations of agents that affect the state or behavior of } \\
\text { member agents and are affected by their member) represented in the } \\
\text { model? }\end{array}$ \\
\hline Observation & $\begin{array}{l}\text { What outputs from the model are needed to observe it internal dynamics } \\
\text { as well as system level behavior? What tools are needed to obtain these } \\
\text { outputs (file outputs, graphs, tables, etc.)? }\end{array}$ \\
\hline
\end{tabular}

In Chapter 4, which details the methodology application case study, the spatially explicit socio-economic model is described using the Overview, Design concepts, and Details $(O D D)$ protocol. All seven elements of this protocol, including design concepts, are also given. 


\section{Chapter 4}

\section{The Upper Thames River basin case study}

The objective of this chapter is to demonstrate the practical implementation of a newly suggested multi-method modeling methodology, and to rationalize all components of the operational Integrated Hydrologic-Socio-Economic Model (IHSEM-UTRB) developed for the Upper Thames River basin case study, located in Southwestern Ontario, Canada. Before discussing the system components, however, we need to identify all the unique characteristics of the selected region.

\subsection{Upper Thames River basin study area}

According to the current Canadian federal and provincial regulations, the government of Ontario has authorized the Upper Thames River Conservation Authority (UTRCA) to administer local water and land resources by balancing the needs of local residents and surrounding environment (UTRCA, 2012). Initially, the UTRCA's main focus was flood control and protection through implementation of purely structural solutions. But, over the years, as the perception of natural resources changed, the UTRCA operations have moved toward a more holistic and integrated ecosystem management approach (UTRCA, 2012). Today, the responsibilities given to UTRCA include environmental planning, managing lands and facilities, soil conservation and forestry, and drinking water source protection.

Unfortunately, numerous natural and socioeconomic trends observed in the basin over the last several decades, such as industrialization, urbanization and changing climate conditions, are expected to place additional pressures on the natural resources in the following period. Having that in mind, the integrated water resources management simulation model is designed to capture the unique characteristics of the local environment by coupling the socio-economic and physical sub-systems. This is implemented with the idea to potentially assist local water authorities, stakeholders, government institutions and individual water users with evaluating different management strategies and policies that the basin may come to use in the near future. 


\subsubsection{Physical conditions in the Upper Thames River basin}

The main Thames river course was formed after the retreat of the Wisconsin Glacier from Ontario around 14,000 years ago (Wilcox et al., 1998). Native inhabitants, Algonquin and Iroquis, firstly named the river Askunessippi. The name was changed to Thames River in 1793 when Governor J. G. Simcoe renamed it after the river in England. Today, the total length of the Thames River is $273 \mathrm{~km}$, with the slope of $1.9 \mathrm{~m} / \mathrm{km}$ for upstream sections, and $0.2 \mathrm{~m} / \mathrm{km}$ for lower and flatter reaches.

As a result of a political agreement and numerous practical reasons, the Thames River basin is divided and managed by two separate conservation authorities responsible for supervision of local water and land resources - Upper and Lower Thames River Conservation Authorities. The Upper Thames River has two main branches. The north branch flows southward through cities of Mitchell and St. Marys and reaches the City of London. Near the city center, the north branch meets the east branch which flows westward through cities of Woodstock, Ingersoll, and east London. The Thames River then flows westward toward Lake St. Clair and enters the lake north of Tilbury. At Byron, the Thames River enters the Lower Thames River Basin. The Upper Thames River Basin is naturally divided into 28 sub-basins, Figure 12.

Annual discharge of the Upper Thames River measured at the Byron station is approximated to $35.9 \mathrm{~m}^{3} / \mathrm{s}$ (Wilcox et al., 1998). In the Upper Thames basin, flow in the river is comprised of $40 \%$ surface water runoff and $60 \%$ base flow. The Upper Thames River basin receives on average approximately $1000 \mathrm{~mm}$ of precipitation annually, Table 5. On average, $60 \%$ of precipitation infiltrates to the ground, evaporates or is lost by evapotranspiration. The remaining $40 \%$ ends up as flow in the river. Within the region, annual precipitation varies by almost 2.5 times from year to year and station to station. This implies significant variations in groundwater recharge rates and stream flows. 
Table 5: Thames Region annual Precipitation, 1950 - 2005 in mm

\begin{tabular}{cccc}
\hline Location & Average & Max (Year) & Min (Year) \\
\hline Chatham & 845 & $1234(1985)$ & $530(1963)$ \\
London & 978 & $1315) 1990)$ & $569(1963)$ \\
Woodstock & 902 & $1264(1996)$ & $542(1953)$ \\
Stratford & 1029 & $1347(1985)$ & $688(1963)$ \\
\hline
\end{tabular}

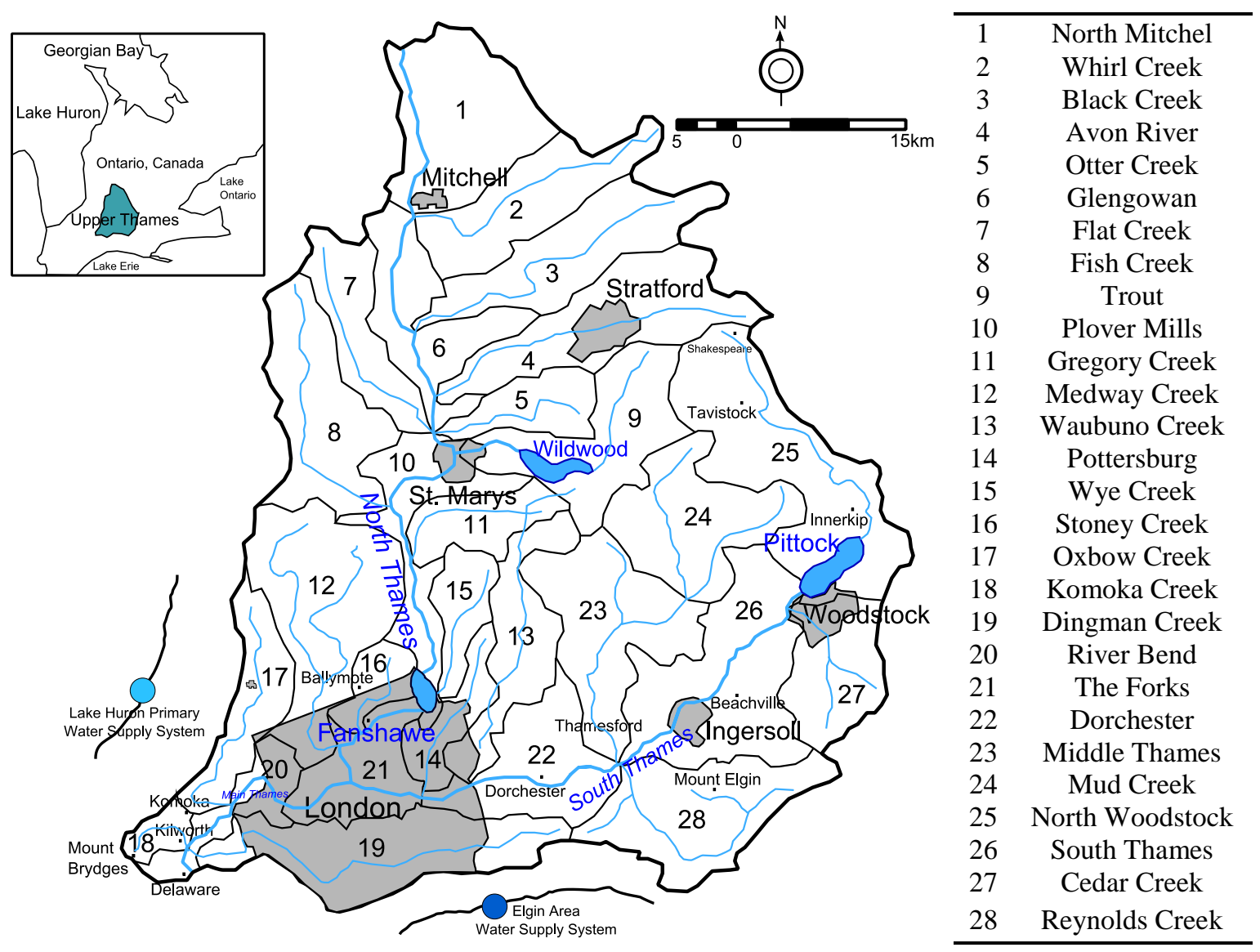

Figure 12: Upper Thames River basin and 28 sub-basins

\subsubsection{Water management infrastructure in the Upper Thames River basin}

Over the years, the UTRCA has established water quality monitoring programs over the basin, regulated the fish stocking programs, and recognized designated heritage sites. In addition, the UTRCA has implemented a number of structures to protect the local municipalities from flooding dykes, municipal drainage schemes, flood and fill line 
regulations. However, the most dominant structures in the Upper Thames River basin are three reservoirs: Wildwood, Pittock, and Fanshawe.

\section{Wildwood reservoir}

Wildwood reservoir was formed by constructing a dam on the Trout Creek upstream of the City of St. Marys. The reservoir was initially proposed in 1948 and finalized in 1965 after three years of construction. In the beginning, the reservoir was designed to provide only flood protection, but shortly after the design was modified to increase low flows and improve the aquatic ecosystem during the dry summer periods. The minimum flow is defined according to the agreement with the Ministry of Environment and Climate Change and then released during the summer months, Figure 13. Currently, the dam reduces flood flows on the Trout Creek by up to 95\% and on the North Thames below St. Marys the flow is reduced by $10 \%$. During the summer months, treated effluents constitute the majority of the baseflow, and additional flows are used to improve the water quality deteriorated by diluted sanitary sewage released from the waste water treatment plants located in the surroundings. Under normal conditions, the reservoir occupies a surface area of 385 hectares. Lake storage in normal conditions available for summer flow augmentation is 1780 hectare-meters, while the maximum spring runoff storage is 2470 hectare-meters (UTRCA, 2012). During the period of spring runoffs, the coarse control of flows from the dam is controlled by four large sluice gates, while much finer control is achieved by three small valves during summer periods. 


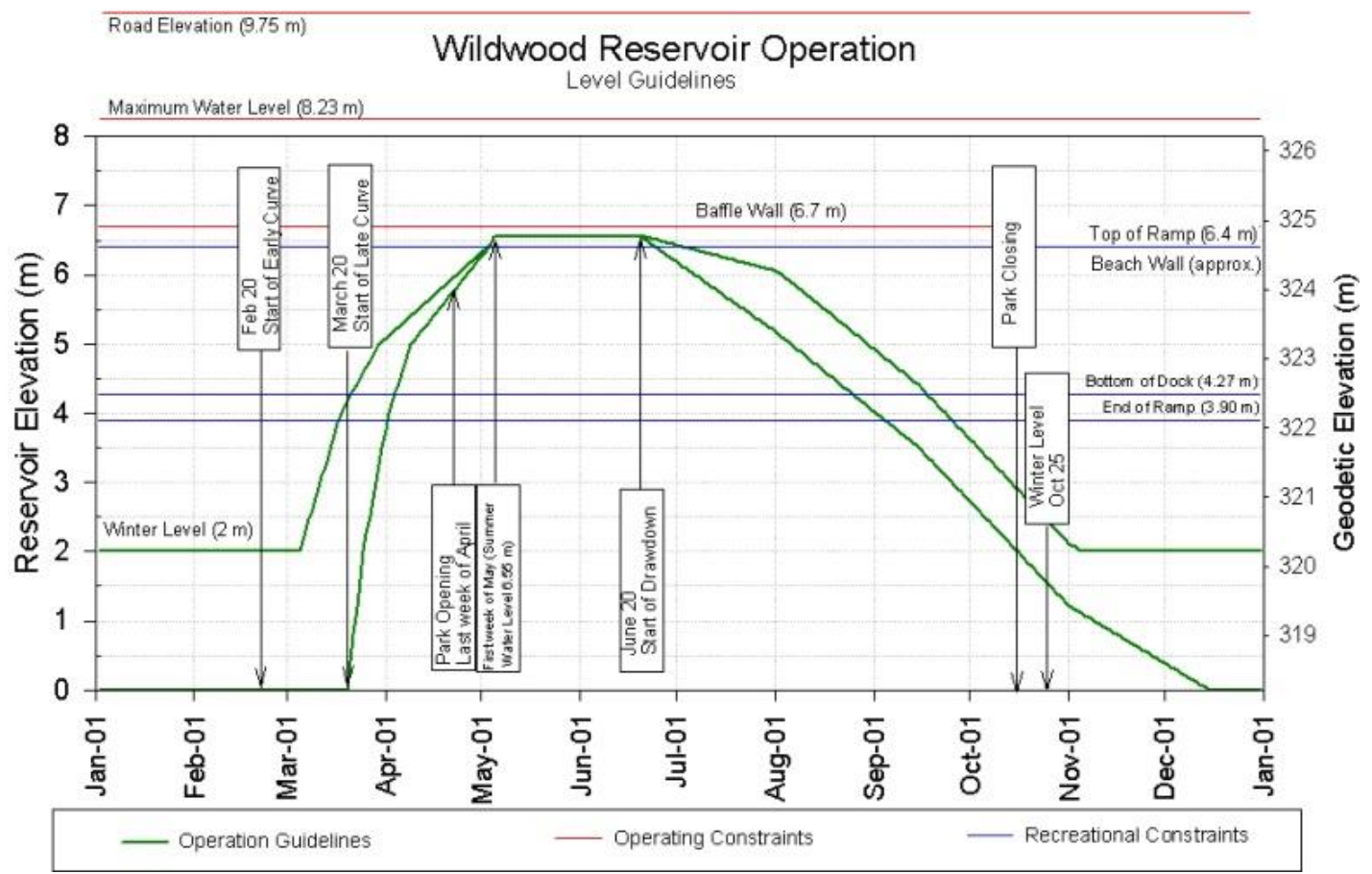

Figure 13: Wildwood reservoir operation guidelines (UTRCA, 2012)

\section{Pittock reservoir}

This $10.3 \mathrm{~km}$ long reservoir was designed to provide flood protection to downstream communities, as well as to improve river flows during dry periods and water quality after pollution caused by sanitary sewage. A minimum volume of water is released during the summer months according to the agreement with the Ontario's Ministry of Environment. The annual operating cycle contains substantial fluctuations in water levels during the year. These fluctuations are used to create the optimal year-round flood protection for downstream communities, and to benefit downstream water quality during the dry summer months, Figure 14. 


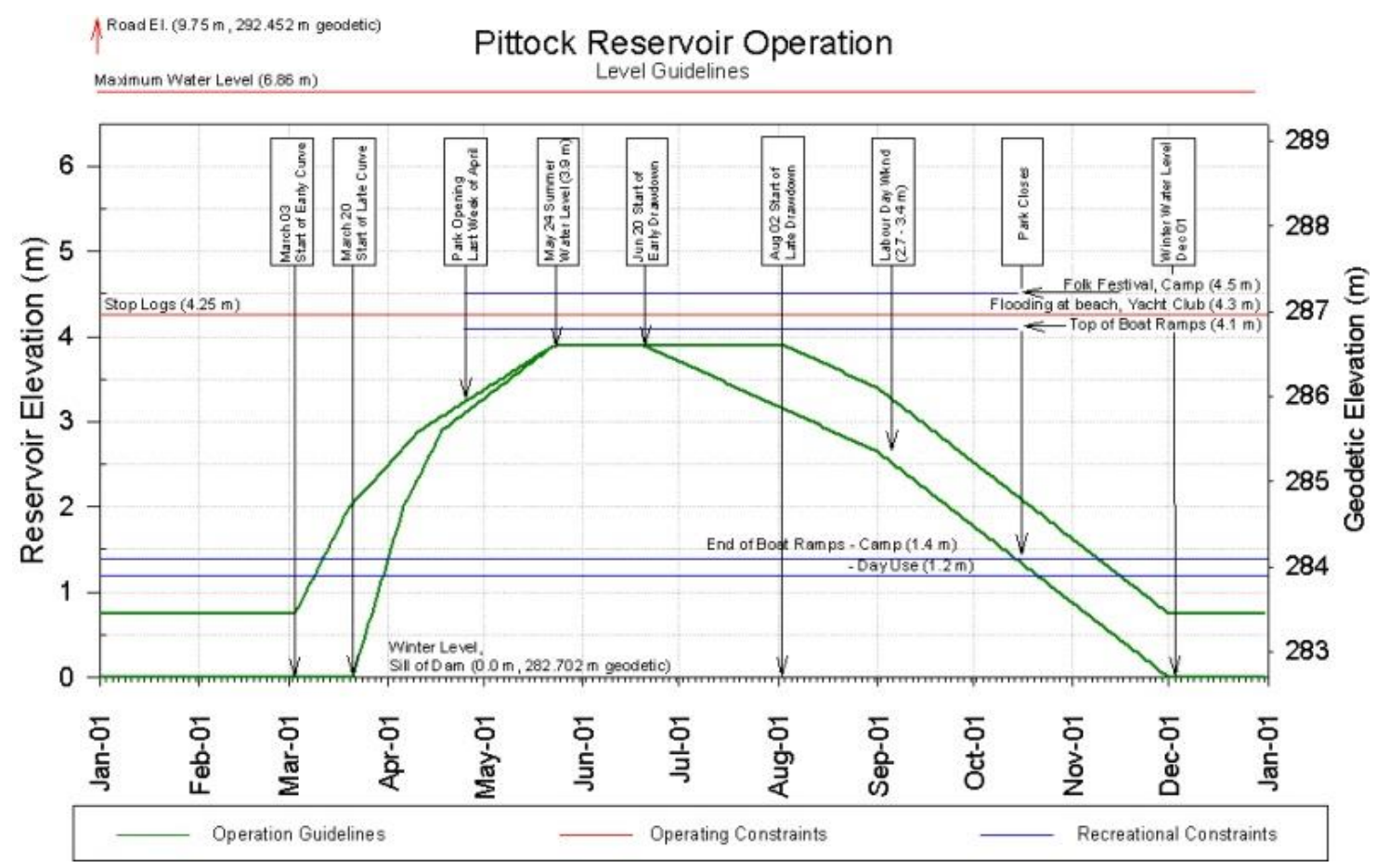

Figure 14: Pittock reservoir operation guidelines (UTRCA, 2012)

\section{Fanshawe reservoir}

The Fanshawe reservoir, constructed between 1950 and 1952, is primarily designed to reduce flooding in the City of London, caused by intensive rainfall and snowmelt events. During the year, released outflow from the reservoir is generally equal to the inflow, except during the flood events. The reservoir is then used to store additional volume of water and release it after the flood event. The total length of the crest of the Fanshawe dam is $625 \mathrm{~m}$. The distance from the crest to river bed is $23.5 \mathrm{~m}$, while the distance from the crest to bedrock is $30.5 \mathrm{~m}$, Figure 15. Maximum total discharge capacity for 6 sluice gates is $3200 \mathrm{~m}^{3} / \mathrm{s}$. A typical summer discharge from the reservoir is $4 \mathrm{~m}^{3} / \mathrm{s}$. In normal conditions, the Fanshawe reservoir stores around 12 billion liters of water. In maximum possible flood conditions, the reservoir can store up to 48 billion liters. The Fanshawe dam also includes the small hydroelectric plant at the base that generates enough power for 300 households (UTRCA, 2012). 


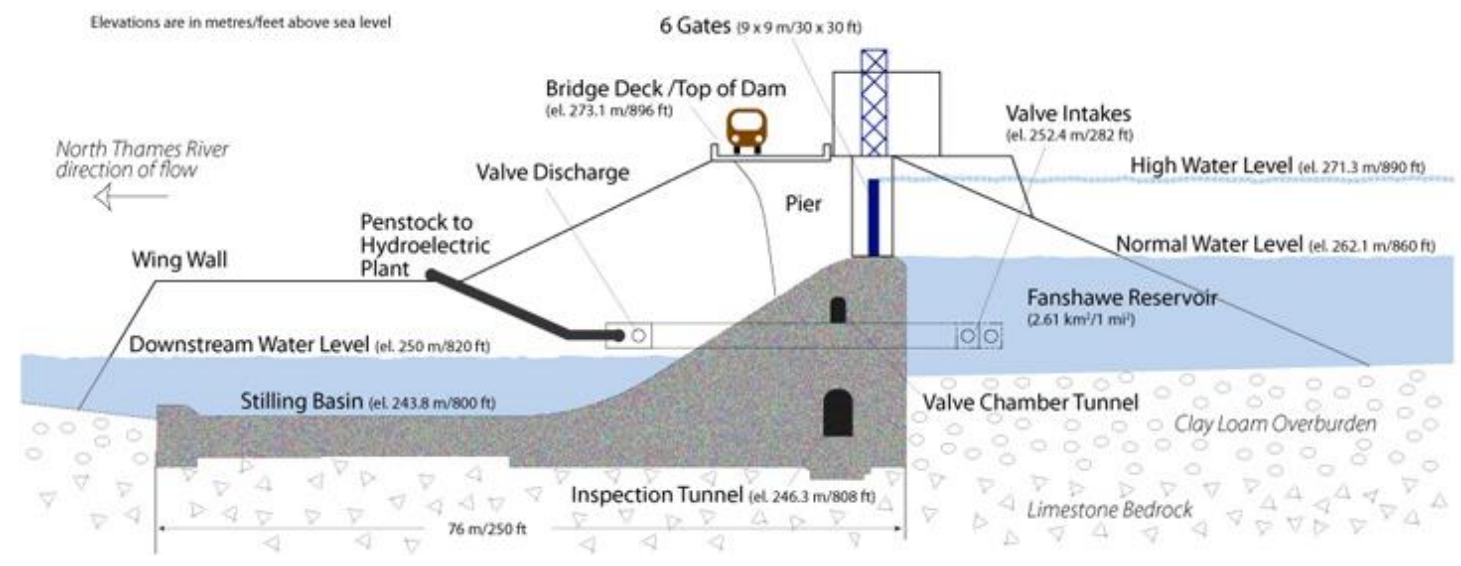

Figure 15: Cross-section of the Fanshawe dam (UTRCA, 2012)

Together with other structural measures, such as dyke systems in the cities of St. Marys and London, the three reservoirs constitute an effective system for flood management and protection. However, floods and droughts are still two major natural hazards in the Upper Thames River basin related to local water resources. $25 \%$ of all floods in the basin occur during March, while 50\% of all floods take place in the period between February and April. Early spring floods are a result of temperature rise and snowmelt (Cunderlick and Simonovic, 2004). Also, floods can occur in the time between December and April due to a combination of snowmelt and intensive precipitation events. The flows in that period can go up to $300 \%$ of mean annual flows. On the other hand, dry conditions are most likely to occur in the period between June and September, while droughts are possible throughout the year. During the summer months, river flows can decrease to $20-30 \%$ of long term annual mean flows.

\subsubsection{Socio-economic setting of the Upper Thames River basin}

The study area covers portions of three counties: Oxford County, Perth County, and Middlesex County. The largest urban centers in the basin are London, Mitchell, St. Marys, Stratford, Ingersoll, Dorchester, and Woodstock, London being the largest, with a population of approximately 366,000. According to the Ministry of Finance, the population of Southwestern Ontario is projected to increase by $18 \%$ in the period between $2006(1,579,400)$ and $2031(1,857,700)$. However, it is expected that particular 
counties, such as Middlesex (22\%), will exceed the regional average (Statistics Canada, 2011).

One of the reasons for the projected population growth is the diverse economy present in the Upper Thames River basin. The main economic strength lies in wide-ranging agricultural activities, from specialized crops to various livestock operations. The three counties the basin resides in (Oxford, Perth, and Middlesex) represent one of the most productive agricultural regions in Canada, supporting a broad range of both specialized and intensive farming operations (Statistics Canada, 2010b). The educational sector is also very strong, and includes widely-recognized educational, medical and research institutions. Manufacturing also contributes significantly to the regional economy, and provides employment for the local population. Numerous automotive and manufacturing facilities are located around the cities of London, Ingersoll and Woodstock. Around 76\% of the total basin area (approximately $3430 \mathrm{~km}^{2}$ ) is nevertheless dedicated to agricultural activities. Over the last 40 years, an occurring trend in UTRB is the conversion of agricultural lands to crop cultivation land use. Urban areas take $10 \%$, forest land $12 \%$ (individual sub-basins range from 5-21\%), while the remaining $2 \%$ go to water and queries, Table 6. Today, agricultural fields, urban development and other land uses fragment existing forest lands into small woodlots. However, since forests have numerous environmental values, such as moderating local climate conditions, one of the main goals pursued by the UTRCA is to have at least $30 \%$ of all sub-basins converted to forest land.

The state of the local environment within the Upper Thames River basin is assessed and presented to the general public by the UTRCA every five years. The report cards grade the basin's surface water quality, forest conditions, physical conditions (such as land use), and give recommendations on how to improve the environmental conditions. This practice has been also followed and implemented by the number of other Ontario's Conservation Authorities. 
Table 6: Land use in the Upper Thames River basin

\begin{tabular}{|c|c|c|c|c|c|c|c|c|}
\hline & Sub-basin & Area & $\begin{array}{c}\text { Area } \\
\text { Lying } \\
\text { Upstream }\end{array}$ & Agriculture & Forest & Urban & $\begin{array}{c}\text { Water } \\
\text { and } \\
\text { Quarry }\end{array}$ & Population \\
\hline [ID] & [Name] & {$\left[\mathrm{km}^{2}\right]$} & {$\left[\mathrm{km}^{2}\right]$} & {$[\%]$} & {$[\%]$} & {$[\%]$} & {$[\%]$} & [2011] \\
\hline 1 & North Mitchel & 173.4 & & 93 & 5 & 2 & 0 & 4,870 \\
\hline 2 & Whirl Creek & 130.2 & & 92 & 7 & 1 & 0 & 2,390 \\
\hline 3 & Black Creek & 138.6 & & 83 & 16 & 1 & 0 & 2,070 \\
\hline 4 & Avon River & 167.9 & & 76 & 11 & 14 & 0 & 32,390 \\
\hline 5 & Otter Creek & 59.2 & & 88 & 10 & 1 & 1 & 740 \\
\hline 6 & Glengowan & 114.2 & 610.1 & 87 & 10 & 2 & 0 & 1,430 \\
\hline 7 & Flat Creek & 90 & & 90 & 10 & 0 & 0 & 600 \\
\hline 8 & Fish Creek & 148.8 & & 91 & 9 & 0 & 0 & 1,500 \\
\hline 9 & Trout & 161.9 & & 78 & 17 & 3 & 2 & 3,600 \\
\hline 10 & Plover Mills & 119.9 & 1299.1 & 74 & 12 & 9 & 2 & 5,670 \\
\hline 11 & Gregory Creek & 59.2 & & 91 & 8 & 1 & 0 & 680 \\
\hline 12 & Medway Creek & 205 & & 83 & 11 & 6 & 0 & 26,040 \\
\hline 13 & Waubuno Creek & 105.1 & & 83 & 12 & 5 & 0 & 7,290 \\
\hline 14 & Pottersburg & 44.7 & & 40 & 17 & 53 & 0 & 25,680 \\
\hline 15 & Wye Creek & 55.7 & & 89 & 9 & 1 & 1 & 1,270 \\
\hline 16 & Stoney Creek & 37.8 & & 69 & 12 & 14 & 5 & 20,240 \\
\hline 17 & Oxbow Creek & 89 & & 84 & 15 & 1 & 0 & 3,210 \\
\hline 18 & Komoka Creek & 21.4 & & 65 & 21 & 10 & 5 & 3,210 \\
\hline 19 & Dingman Creek & 170.1 & & 64 & 14 & 21 & 0 & 7,4620 \\
\hline 20 & River Bend & 58.3 & 3362.4 & 49 & 24 & 19 & 8 & 29,600 \\
\hline 21 & The Forks & 88.1 & 2993.8 & 4 & 13 & 78 & 5 & 182,800 \\
\hline 22 & Dorchester & 137.2 & 1194.8 & 68 & 21 & 9 & 2 & 16,870 \\
\hline 23 & Middle Thames & 171.2 & 156.5 & 85 & 13 & 1 & 1 & 3,170 \\
\hline 24 & Mud Creek & 156.5 & & 87 & 12 & 1 & 0 & 2,460 \\
\hline 25 & North Woodstock & 242.9 & & 80 & 13 & 6 & 1 & 21,500 \\
\hline 26 & South Thames & 226.8 & 338 & 77 & 11 & 10 & 2 & 20,870 \\
\hline 27 & Cedar Creek & 95.1 & & 73 & 12 & 14 & 1 & 20,340 \\
\hline \multirow[t]{2}{*}{28} & Reynolds Creek & 152.5 & & 87 & 12 & 1 & 0 & 2,125 \\
\hline & Total: & 3421 & & 76 & 12 & 10 & 2 & 366,000 \\
\hline
\end{tabular}

\subsubsection{Water allocation in the Upper Thames River basin}

The main consequences of growing population, urbanization and strong economic growth are numerous conflicting interests that pressure local natural resources. The Upper Thames River Basin has initiated a water allocation and water use management system in order to regulate surface and groundwater withdrawal. The main objective behind this system is to balance local water resources that benefit all social, economic and environmental goals. These goals are defined at different levels, from local, sub-basin, basin, regional, to the provincial level. To do so, the UTRCA has set up three levels of 
water budgeting for the Upper Thames River Basin. Tier 1 determines the water budget on the level of the entire basin. Previous analysis shows that a more detailed Tier 2 water budget assessment is required for five sub-basins (Cedar Creek, Reynolds Creek/Thames River, North Thames/Medway River, Middle Thames River, and Black Creek/Avon River). Water use rates used in the study are based on the Permit to Take Water Database for this particular region. According to the Section 34 of the Ontario Water Resources Act (OWRA), Permit to Take Water (PTTW) database, maintained by the responsible Conservation Authority, in this case the Upper Thames River Conservation Authority (UTRCA), holds a record of individual permit holders in the study area. According to the provincial Ministry of Environment and Climate Change, all users that pump more than 50,000 liters per day require a special permission (the Permit to Take Water), except in the case when water is used for agricultural livestock. Once the permit is issued, a water user is registered in the Permit to Take Water Database. Apart from the information on specific water users, this database contains information about the pumped water source (groundwater, surface water or both), the spatial location of pumping, determines the particular type water use, and defines the maximum permitted water taking for each user. Table 7 presents the number of Permits to Take Water (PTTW) per sector in the Thames River basin and their maximum annual permitted volumes.

This table shows that $33 \%$ of all permits are in the agricultural sector, but the total maximum volume permitted for agriculture is about $5 \%$. The difference is a result of the seasonal nature of water takings associated with crop irrigation. On the other side, the dewatering sector has $6 \%$ of permits, but takes approximately $33 \%$ of permitted volume. The difference is caused by the need for continuous water withdrawals over the year. Dewatering activities include removal of water from solid material or soil by wet classification, centrifugation, or filtration. 
Table 7: Number of water permits by sector in Thames River basin

\begin{tabular}{|c|c|c|c|c|c|}
\hline $\begin{array}{l}\text { Water Taking } \\
\text { Sector }\end{array}$ & Water Use & $\begin{array}{l}\text { Number } \\
\text { of } \\
\text { Permits }\end{array}$ & $\begin{array}{l}\text { Percent } \\
\text { of Total } \\
\text { Permits }\end{array}$ & $\begin{array}{c}\text { Total } \\
\text { Annual } \\
\text { Maximum } \\
\text { Volume } \\
{\left[\mathrm{m}^{3} / 10^{6}\right]} \\
\end{array}$ & $\begin{array}{l}\text { Percent of } \\
\text { Total } \\
\text { Maximum } \\
\text { Volume } \\
\text { Permitted } \\
\end{array}$ \\
\hline Agricultural & $\begin{array}{c}\text { Field and pasture crops, } \\
\text { fruit orchards, market } \\
\text { gardens/flowers, nursery, } \\
\text { sod farm, tender fruit, } \\
\text { tobacco }\end{array}$ & 300 & $33 \%$ & 36,043 & $5 \%$ \\
\hline Commercial & $\begin{array}{l}\text { Aquaculture, bottled } \\
\text { water, golf course } \\
\text { irrigation, mall/business, } \\
\text { snowmaking }\end{array}$ & 158 & $17 \%$ & 32,116 & $4 \%$ \\
\hline Construction & $\begin{array}{l}\text { Construction, road } \\
\text { building }\end{array}$ & 10 & $1 \%$ & 947 & $0.1 \%$ \\
\hline Dewatering & $\begin{array}{c}\text { Construction, pits and } \\
\text { quarries }\end{array}$ & 52 & $6 \%$ & 241,193 & $33 \%$ \\
\hline Industrial & $\begin{array}{l}\text { Aggregate washing, } \\
\text { cooling water, food } \\
\text { processing, pipeline } \\
\text { testing, power production }\end{array}$ & 92 & $10 \%$ & 222,480 & $30 \%$ \\
\hline Institutional & Hospitals & 1 & $0 \%$ & 183 & $0.0 \%$ \\
\hline Miscellaneous & $\begin{array}{c}\text { Dams and reservoirs, heat } \\
\text { pumps, wildlife } \\
\text { conservation }\end{array}$ & 57 & $6 \%$ & 59,081 & $8 \%$ \\
\hline Recreational & Aesthetics & 12 & $1 \%$ & 539 & $0.1 \%$ \\
\hline Remediation & $\begin{array}{l}\text { Groundwater, other } \\
\text { remediation }\end{array}$ & 6 & $1 \%$ & 51 & $0.0 \%$ \\
\hline Water Supply & $\begin{array}{l}\text { Campgrounds, communal, } \\
\text { municipal, water supply }\end{array}$ & 217 & $24 \%$ & 148,510 & $20 \%$ \\
\hline
\end{tabular}

However, the Permits to Take Water (PTTW) database does not include unpermitted water takings. For the purpose of this study, two categories of unpermitted water takings 
were analyzed: unserviced domestic water use and agricultural water use. Unserviced domestic water use was estimated by using the number of people living in each municipality within unserviced areas determined by census data and assumed per capita water use of $185 \mathrm{~L} / \mathrm{Day}$. On the sub-basin level, it was assumed that the unserviced population was equally distributed over the municipality. As a result, unserviced pumping rates were determined for the sub-basins based on the percentage of the municipality in a sub-basin. In contrast, unpermitted agricultural water use was calculated by defining the number of livestock in each census subdivision and the amount of water required for each type of livestock. Under the assumption that the livestock is evenly distributed over the census subdivision area, the total water use for each sub-basin was based on the percentage area of the census subdivision in the basin area. In addition to these two categories, the rates of unpermitted water takings include the averaged dewatering activities on the level of each sub-basin.

Urban centers in the Upper Thames River basin mainly get water for municipal needs from two water supplying systems: The Lake Huron Primary Water Supply System and The Elgin Area Water Supply System. From the water treatment plant located near the village of Grand Band on the South Huron Lake, the Lake Huron Primary Water Supply System services the municipalities of London, Lambton Shores, North Middlesex, South Huron, Bluewater, Middlesex Centre, Lucan-Biddulph and Strathroy-Caradoc. The current treatment capacity is 340 million liters per day and serves a population of approximately 350,000 people. The Elgin Area Primary Water Supply System services the municipalities of St. Thomas, London, Aylmer, Bayham, Central Elgin, Malahide and Southwold from a water treatment plant located east of the village of Port Stanley in Central Elgin. The plant has a current treatment capacity of 91 million liters per day and serves a population of approximately 112,000 people (Thames - Sydenham and Region Drinking Water Source Protection, 2014). 


\subsection{Components of the Integrated Hydrologic-Socio- Economic Model for the Upper Thames River basin (IHSEM-UTRB)}

According to the previously defined multi-method modeling framework, the integrated model incorporates a number of components that have appropriately defined roles. The following sections explain all necessary details of all implemented components for the Upper Thames case study, starting with the spatial database, followed by the spatially semi-distributed continuous hydrologic model, and finalizing with the spatially explicit socio-economic agent-based model.

\subsubsection{Component 1: Spatial database}

\subsubsection{Shapefiles}

Due to the requirements of used agent-based modeling platforms and the requirements of the tight coupling strategy, this work utilizes the shapefile format to store all relevant information related to the Upper Thames River basin case study (instead of using the advanced geodatabase). All shapefiles are directly linked to Component 3 and represent the source of information for the socio-economic model. Also, raster datasets are used to represent spatially continuous data, such as land use categories, elevation, and county and sub-basin areas. Descriptions of all shapefiles are given bellow, while their lists of attributes are given in Appendix A. All described spatial databases, both vector and raster, are retrieved from following sources: The Ministry of Environment and Climate Change (Ontario, 2014) and Scholars Geo Portal (Scholarsgeoportal, 2014).

\section{UTSubbasins.shp}

This shapefile spatially describes the boundaries of the Upper Thames River basin and delineates its 28 sub-basins. Each sub-basin contains a unique identifier (OBJECTID) and other relevant information that is used by the model, such as total area (SB_AREA), percentage of occupied area within a county (AREA_PERC), and quantities of unpermitted private (PRIVATEUSE) and agricultural water use (AGRIUSE). This vector 
file is also used to extract the agents that represent responsible decision makers on the sub-basin level used by the agent-based model.

\section{UTUrbanCenters.shp}

This dataset represents eight major urban centers in the UTRB (London, Mitchell, St. Marys, Woodstock, Ingersoll, Dorchester, Stratford, and Komoka). Every item in the list holds a unique identifier (CITY_ID), is spatially defined, and contains a set of socioeconomic attributes used by the socio-economic sub-models, such as population at the first time step in simulation (CSD_POP00).

\section{UTHydrography.shp}

This shapefile describes hydrographic network in the Upper Thames River basin.

\section{UTGaugeStations_2014.shp}

Spatial locations of gauging stations in the UTRB and their properties are stored in this shapefile dataset.

\section{UTCounties.shp}

The Upper Thames River basin occupies portions of three counties (Oxford, Perth and Middlesex), and their boundaries are described in this shapefile. This dataset is also used to define the agents that represent the decision makers on the county level.

\section{UTAgriculturalPPTW_2014.shp}

This shapefile contains information on the agricultural users extracted from the Permit to Take Water (PTTW) database in the UTRB. In addition to their spatial locations, this datasets defines the maximum permitted quantities for taking (MAXL_DAY and DAYS_YEAR), their specific agricultural purpose (SPURPOSE), and describes the dates when the permit was issued and when it expires in date format (ISSUEDDATE and EXPIRYDATE). However, due to the technical limitations of used agent-based modeling environment these date values are converted to a specific format and stored in attributes 
(ISSUE_TICK and EXPIRY_TIC). OBJECTID represents a unique identifier for all individual agricultural water users. LINK_TO_SW attribute is used to create a direct link with responsible manager on sub-basin level, based on the location of particular water user.

\section{UTCommercialPPTW_2014.shp}

This dataset contains information on the commercial water users extracted from the Permit to Take Water (PTTW) database. OBJECTID represents a unique identifier for every item in the list. This datasets defines individual spatial location for all commercial water users, the maximum permitted quantities for taking (MAXL_DAY and DAYS_YEAR), and describes the dates when the permit was issued and when it expires in date format (ISSUEDDATE and EXPIRYDATE). Similarly to the agricultural shape file these values in date format are converted to a specific format and stored in attributes ISSUE_TICK and EXPIRY_TIC.

\section{UTIndustrialPPTW_2014.shp}

This shapefile contains information on the industrial water users extracted from the Permit to Take Water (PTTW) in the UTRB. OBJECTID represents a unique identifier for all individual industrial users, while other attributes all identical to the ones in agricultural and commercial shape files. SPURPOSE attribute defines a specific industrial purpose for each individual user.

\section{UTWaterSupplyPPTW_2014.shp}

This shapefile contains information on the sources of municipal water supply extracted from the Permit to Take Water (PTTW) database. OBJECTID represents a unique identifier, and each item in the list contains the information on maximum daily and annual capacity (MAXL_DAY and DAYS_YEAR). SOURC_CITY attribute links a particular water source in the list with an urban center. 
UTPrimaryWaterSupply_2014.shp

All relevant information about the two water supplying systems in the UTRB is given in this dataset. This dataset is extracted from the Permits to Take Water (PTTW) database. OBJECTID represents a unique identifier, while all other attributes describe technical properties of the two systems, such as the total daily and annual capacities.

\subsubsection{Raster datasets}

In addition to the vector data stored as shapefile datasets, spatially continuous data is stored in the raster image format. Developed integrated water resources management model for the Upper Thames River basin includes following raster datasets:

\section{Land use dataset}

This dataset contains information on The Upper Thames River basin land use and contains 39 land use categories for all cells. Since the simulation starts on the January 1st 2000, the same date is taken to initiate the land use category data. Each cell can contain only one value of current land use category, and this value is updated throughout the simulation, Table 8 . 
Table 8: Land-use categories in the Upper Thames River basin and their identification number

\begin{tabular}{|c|c|}
\hline $\begin{array}{l}\text { Land use } \\
\text { Category ID }\end{array}$ & Description \\
\hline 1 & Built up areas - London \\
\hline 2 & Built up areas - Dorchester \\
\hline 3 & Built up areas - Stratford \\
\hline 4 & Built up areas - Mitchell \\
\hline 5 & Corn system \\
\hline 6 & Grazing system \\
\hline 7 & Hay system \\
\hline 8 & Tobacco system \\
\hline 9 & Mixed system \\
\hline 10 & Mixed system \\
\hline 11 & Continuous row crop \\
\hline 12 & Water \\
\hline 13 & Swamp, marsh or bog \\
\hline 14 & Woodland \\
\hline 15 & Idle agricultural land (over 10 years) \\
\hline 16 & Built up areas - Upper Thames Basin \\
\hline 17 & Pasture system \\
\hline 18 & Extensive field vegetables \\
\hline 19 & Market gardens \\
\hline 20 & Orchard \\
\hline 21 & Extraction pit and quarries \\
\hline 22 & Recreation \\
\hline 23 & Idle agricultural land (5-10 Years) \\
\hline 24 & Nursery \\
\hline 25 & Pastured woodland \\
\hline 26 & Reforestation \\
\hline 28 & Built up areas - Komoka \\
\hline 29 & Cherries \\
\hline 30 & Sod farm \\
\hline 31 & Berries \\
\hline 32 & Built up areas - St.Marys \\
\hline 33 & Built up areas - Thamesford \\
\hline 34 & Built up areas - Woodstock \\
\hline 35 & Extraction top soil removal \\
\hline 36 & Built up areas - Ingersoll \\
\hline 37 & Built up areas - Embro \\
\hline 38 & Built up areas - Tavistock \\
\hline 39 & Built up areas - Innerkip \\
\hline
\end{tabular}




\section{Sub-basins dataset}

The Upper Thames River basin contains 28 sub-basins, and each patch in the model belongs to only one sub-basin, Table 9.

Table 9: List of 28 sub-basins in the Upper Thames River basin and their identification numbers

\begin{tabular}{cc}
\hline Sub-basin ID & Sub-basin Name \\
\hline 1 & North Mitchel \\
2 & Whirl Creek \\
3 & Black Creek \\
4 & Avon River \\
5 & Otter Creek \\
6 & Glengowan \\
7 & Flat Creek \\
8 & Fish Creek \\
9 & Trout Creek \\
10 & Plover Mills \\
11 & Gregory Creek \\
12 & Medway Creek \\
13 & Waubuno Creek \\
14 & Pottersburg \\
15 & Wye Creek \\
16 & Stoney Creek \\
17 & Oxbow Creek \\
18 & Komoka Creek \\
19 & Dingman Creek \\
20 & River Bend \\
21 & The Forks \\
22 & Dorchester \\
23 & Middle Thames \\
24 & Mud Creek \\
25 & North Woodstock \\
26 & South Thames \\
27 & Cedar Creek \\
28 & Reynolds Creek \\
\hline &
\end{tabular}

Municipalities dataset

Portion of three counties located in the Upper Thames RB are represented in this dataset, Table 10. Each cell belongs to only one county. 
Table 10: Three counties in the Upper Thames River basin and their identification numbers

\begin{tabular}{cc}
\hline County ID & County Name \\
\hline 1 & Oxford County \\
2 & Middlesex County \\
3 & Perth County \\
\hline
\end{tabular}

Digital Elevation Model (DEM) dataset

Each patch contains a value that defines the elevation in meters above sea level.

Due to the requirements of the selected agent-based modeling environment described in the latter text, these four raster datasets are converted to ASCII format.

\subsubsection{Component 2: Spatially semi-distributed continuous hydrologic model}

In this case study a spatially semi-distributed continuous hydrologic model is applied to describe long-term hydrologic processes in the Upper Thames River basin, Cunderlick and Simonovic $(2004,2005)$. In order to analyze interactions between the socioeconomic and hydrologic systems in the UTRB, Prodanovic (2007) uses system dynamics simulation method to represent local socio-economic environment and couples it with the continuous hydrologic model. Chosen coupling strategy requires that both, the hydrologic model modules and system dynamics socio-economic model must be executed in the same programming language. For that reason, the structure of HEC-HMS model is converted into Java code. Due to the functionalities of selected agent-based modeling environment detailed in the following section, the existing Java code of the continuous hydrologic model is accustomed to create a new Netlogo extension. Details on Netlogo programming environment and Netlogo's Extensions are given in the next chapter, while all coding details of newly created extension are showed in Appendix B.

The hydrologic model schematic developed for the Upper Thames River basin is presented in Figure 16. 


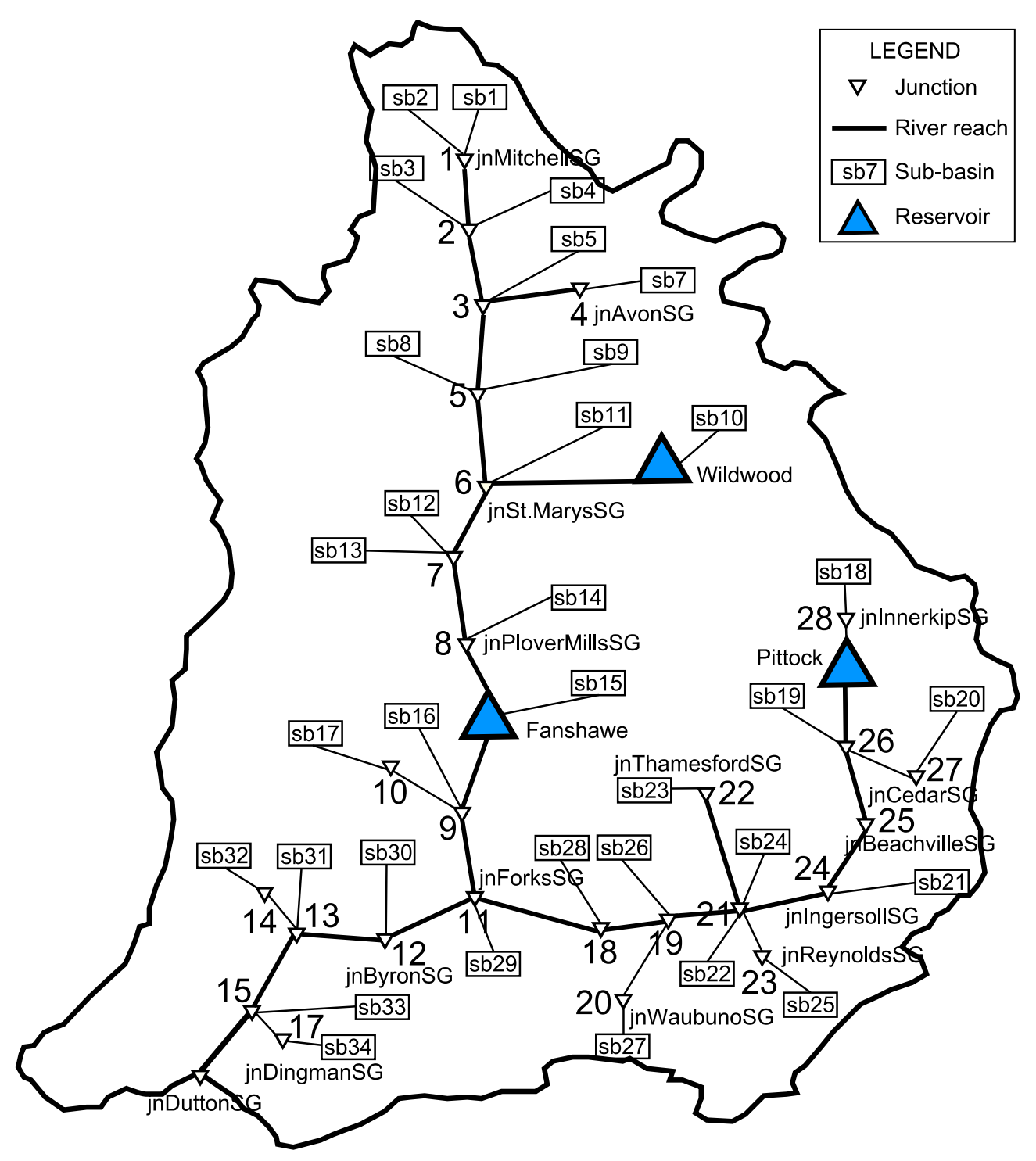

Figure 16: Upper Thames RB Hydrologic model schematics

This model includes twenty-one river reaches and represents all three reservoirs in the system. However, despite the fact that the response of the UTRB is very sensitive to operational procedures of all three reservoirs, applied modules in the hydrologic model, particularly the modified puls method, cannot adequately represent the operation for three reservoirs. Applied methods can only approximate the management practices, and, therefore, this representation of reservoir operations is probably the most important limitation of this procedure. 
Different set of parameters are used to characterize seasonal variations between summer and winter months. All parameters are described by Cunderlick and Simonovic (2004). The model is calibrated for the daily data in the period between November 1979 and October 1988, while the model verification is done for the period between November 1988 and October 1997. The calibration procedure is based on spatially and temporarily interpolated precipitation. Figure 17 illustrates the simulation results of a calibrated model for three flow-gauging stations (Mitchell, St. Marys and Byron). The obtained results show that this continuous model manages to capture regional long-term hydrologic behavior. However, it is concluded that this model tends to underestimate total stream flow volumes by 10-15\%, Cunderlick and Simonovic (2004).

Sensitivity analysis of model parameters has showed that the flood magnitude is sensitive to Clark's storage coefficient and parameters that describe physical properties of the soil (maximum soil infiltration rate, and depths of tension zone and soil profile storage) Cunderlick and Simonovic (2004). In terms of total stream flow volumes, the model is mostly sensitive to the soil moisture accounting parameters that describe underground soil layers. 

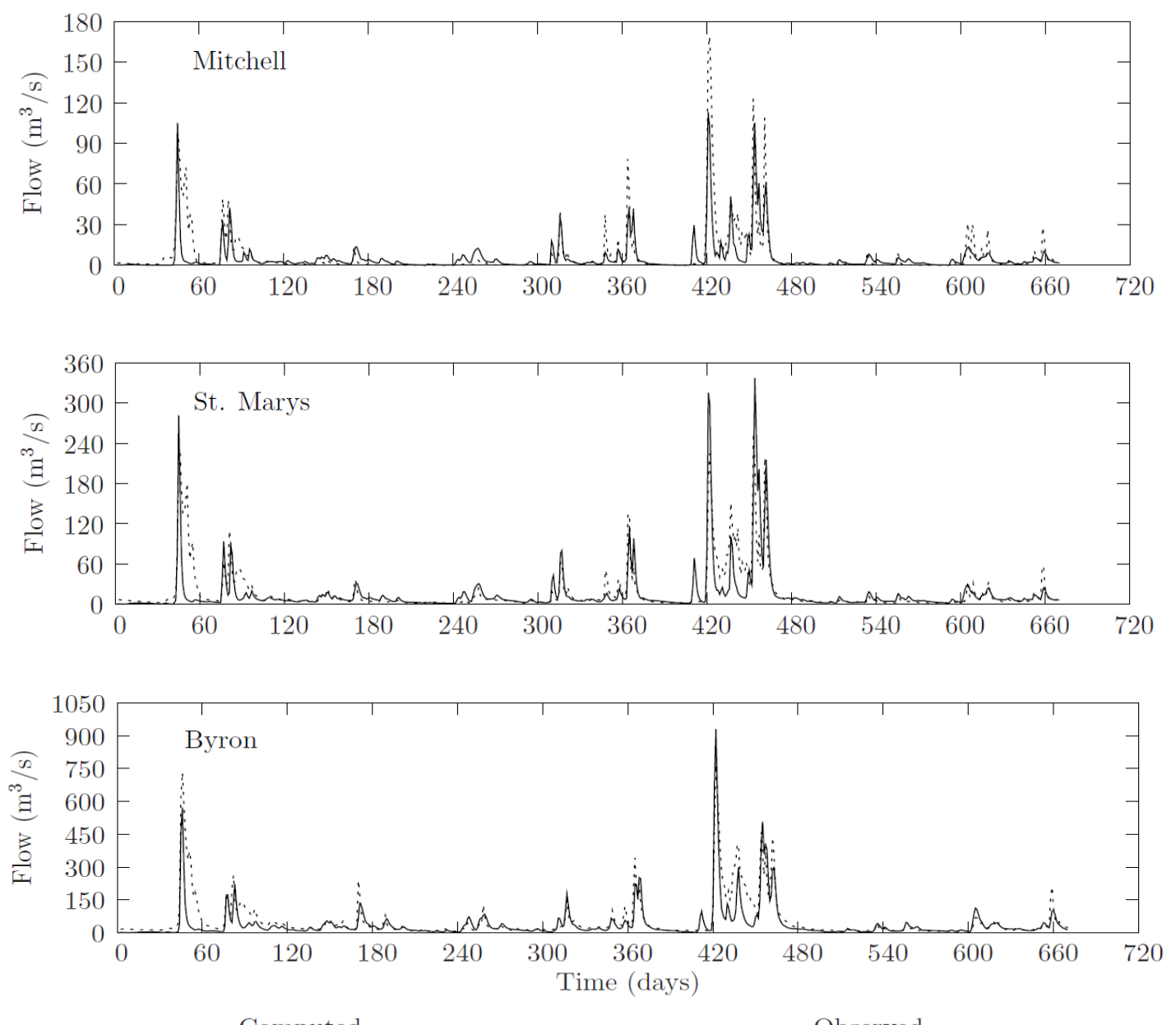

Computed

Observed …...

Figure 17: Hydrologic model calibration results 


\subsubsection{Component 3: Spatially explicit socio-economic agent-based model}

The main principles of integrated water resources management point out that wellbeing of people and further socio-economic development of complex societies strongly depend on natural resources. The main principles also stress that only a holistic and systems approach can help understanding dependencies between socio-economic systems and natural environment. Therefore, in this stage, an agent-based model is developed to represent feedback processes between the natural resources (water and land), hydrological properties of the basin, implemented infrastructure, and complex socioeconomic environment. However, before the actual model implementation, a modeler needs to select the optimal platform for agent-based modeling. Currently, a number of different modeling platforms can be used for the development of agent-based models, such as RePast (North et al., 2013), Swarm (Swarm, 2014), Mason (Luke et al., 2004), Anylogic (AnyLogic, 2015), StarLogo (OpenStarLogo, 2015), and Netlogo (Wilensky, 1999). According to Heppenstall et al. (2012), the process of selection of an appropriate modeling platform is based on two sets of criteria: (i) general criteria and (ii) model specific criteria. General criteria compares the straightforwardness of model development process within the platform, size of the community using the platform, technical support, programming language the system is implemented in, regular platform maintenance and updates, availability of technical documentation and demonstration models. On the other hand, model specific criteria strongly depend on the properties of a system being modeled, such as number of agents that can be represented in the model, ability to represent multiple organizational/hierarchical levels of agents, variety of model environments (network, raster, or vector), management of spatial relationships between agents, and agents and their environment, available mechanisms for scheduling and sequencing events, etc. Other important aspects that contribute the decision process are licensing policy, openness of the source code, shareware, or proprietary options. The key advantage of open source platforms is the transparency of internal program structure, and potential for all users to modify the platform according to their needs. 
After weighing all the criteria, Netlogo (Wilensky, 1999) programming environment has been selected for the agent-based component development in the presented research. Compared with other modeling platforms, Netlogo offers a number of advantages that are important for this particular case study. Firstly, its source code is publicly available, which makes it suitable for further improvements and modifications according to specific model requirements. Secondly, an extensive library of developed models comes with the desktop installation of this modeling platform that can be used for the learning process. Moreover, a list of introductory to advanced level tutorials is provided by numerous Netlogo modeling community. Finally, the most important reason for choosing Netlogo lies in its ability to create external extensions. This particular feature is used to develop dynamic data exchange links between all three model components.

Netlogo is a programming language for the development of agent-based models. This particular platform has evolved on the foundation of the Logo programming language developed in 1960's for the educational purposes (Wilensky, 1999). Netlogo brings several characteristic concepts. Like in any other agent-based modeling platform, the basic entity in Netlogo is an agent. Netlogo contains four predefined types of agents: the observer, patches, turtles, and links. The observer is defined as the single global instance that delivers global variables and has direct access to the attributes of all other agents. This agent gives commands to other agents and the environment through a command line. Patches (or cells) represent immobile agents with distinctive locations in space that do not change in time. All patches together comprise the model environment, which is the two-dimensional extent of the world. Each patch has a set of predefined variables, such as x-location, y-location, color, or label. However, in contrast to the Cellular Automata (CA) modeling method, patches can store a significant number of variables defined by the user. In Netlogo programming language, mobile agents have a unique name - turtles. Depending on the model requirements, they can move through the world comprised of patches or can be spatially static. Just like patches, turtles have pre-defined set of variables that characterize them, such as x-location, y-location, id-number, shape, label, etc. Also, turtles can contain an extensive number of user-defined variables. Turtles with common properties and identical set of characteristic attributes can be grouped into breeds. Different breeds declare different types of turtles. The final type of agents defined 
in Netlogo is link. Links define communication corridors and dependences between two or more turtles since all agents can communicate and interact with each other. In contrast to patches and turtles, links do not contain any spatial attributes. Every link must have defined two parameters: starting turtle and ending turtle. Links are commonly used to represent social environment in social dynamic simulations. Netlogo includes two more unique characteristic concepts: commands and reporters. Commands can be described as instructions given to agents, while reporters only calculate certain value and return it for further utilization and representation. Netlogo contains a library of more than 400 already predefined commands and reporters, also known as primitives.

However, from the perspective of coupling the agent-based component with both spatial database and hydrologic models, the most important advantage of Netlogo are extensions. Netlogo modeling platform allows for extension of the primitives (commands and reporters) since Netlogo's extension API offers a way to extend the language by adding user-defined primitives. Extensions can be written in object-oriented programming languages, such as Java or Scala. Initially, Netlogo modeling platform enables importing image files that can be used to represent the environment and thus facilitates the development of spatially explicit models. Latest updates of internal structure and development of GIS extension, allows Netlogo to import both raster (in the form of ASCII files) and vector data (Shapefiles). This important feature allows creation of agents with exact spatial locations as attributes. Also this extension enables a direct link between geographic feature attributes and Netlogo's objects, such as patches or agents. The Netlogo's Extension feature is thus used for tight coupling of agent-based spatially explicit socio-economic model and spatially semi-distributed hydrologic model.

In the following section, previously presented 7 elements of overview, design concepts, and details (ODD) protocol are used to document all details of the spatially explicit socioeconomic agent-based model developed for the Upper Thames River basin case study, Table 3. Since the agent-based model is developed in Netlogo modeling environment, all model elements are described using Netlogo's terminology. 


\subsubsection{Model purpose}

The socio-economic agent-based model is developed to analyze the utilization of natural resources by numerous users within the Upper Thames River basin. On one side, this model simulates the effects of availability of local resources for recognized system actors, while on the other, it also examines the effects that actions of system actors have on the environment, in this case - hydrologic regime. This socio-economic model describes the dependencies between the most dominant socio-economic entities and the local natural resources by assuming the strong relationship between the water demand and socio-economic activities. Typically, analysis of dependencies between socioeconomic and physiographic environment is based on a water budget or water balance models. A water balance counts the surface and groundwater availability and variability as a function of local physiographic and hydrologic conditions. Also, the water budget at a certain spatial location fundamentally depends on the existing water use and the projected demand. The water balance defines the amount of water that can be sustainably allocated without causing interference among users, conflict between extractive and instream uses, or disruption to ecosystem functions. One of the major tasks for water managers is evaluation of risk factors related to potential over-allocation. Following the basic principles of integrated water resources management and due to the properties of hydrologic cycle, the water budget analysis and allocation policies are typically defined on the level of river basins, sub-basins, or particular aquifers.

Therefore, this spatially explicit agent-based model is designed to address two main questions:

1. How do simulated socio-environmental system and its individual entities develop and respond to changing physical and climate conditions, and what model indicators warn of the system vulnerability?

2. How socio-economic activities affect the hydrologic cycle in the Upper Thames River Basin? 
The main goals of this model are:

(a) to assist in better understanding of the complex dynamics of socio-economic and environmental systems; and

(b) to assess quantitative indicators of system sustainability.

The model examines the linkages between different socio-economic processes, such as population dynamics, industrial and agricultural production, reforestation and deforestation, urbanization, and their direct consequences on the local environment.

\section{How this model works?}

The model user takes the role of a decision maker and interactively controls all major socio-economic activities within the basin through a set of switches, sliders and choosers available at the graphical user interface. The main window shows the current conditions of the basin in a map form, Figure 18. The user selects via switches what particular information should be shown on the map. Sliders and choosers define all parameters used by different socio-economic sub-models and they are presented in details in the following sections. Before the simulation is initiated, the user needs to define the climatic conditions in the basin by choosing one of three available climate scenarios (historic, wet, or dry). Each condition represents a specific scenario used for the system analysis. All recognized water users have individual water demand defined based on their specific purpose and respective needs. Water demand also changes over the season and this is especially important for agricultural water users. Based on the taken quantities of water, each agent generates certain economic revenue (commercial, agricultural, and industrial) calculated by economic sub-models. The amount of water they consume is directly proportional to how much profit they make. Each individual water user consequently contributes to the economy on a higher basin level (system level). At the same time, responsible managers (on sub-basin and county levels) monitor the water balance based on the inputs from the hydrologic model and registered water consumption. Initially, the water abundance can sustain the water demand, but, depending on the model user inputs, this can lead to increased water demand and economic revenue. On one side, this is desired from the economic point of view, but is extremely uncertain from the 
environmental standpoint. Also, the model analyzes the dependence between socioeconomic environments and realized infrastructural elements in the system. It compares the quantities of water provided by the two water-supplying systems and accordingly updates the population dynamics sub-model. Some sub-models have direct spatial implications which influence the current land use practices in the basin. Finally, the hydrologic sub-model takes current land use change into account and analyses the resulting hydrologic regimes. To observe the social and economic state of the system and the resulting hydrologic regimes, a set of plots and monitors is provided by the model. 


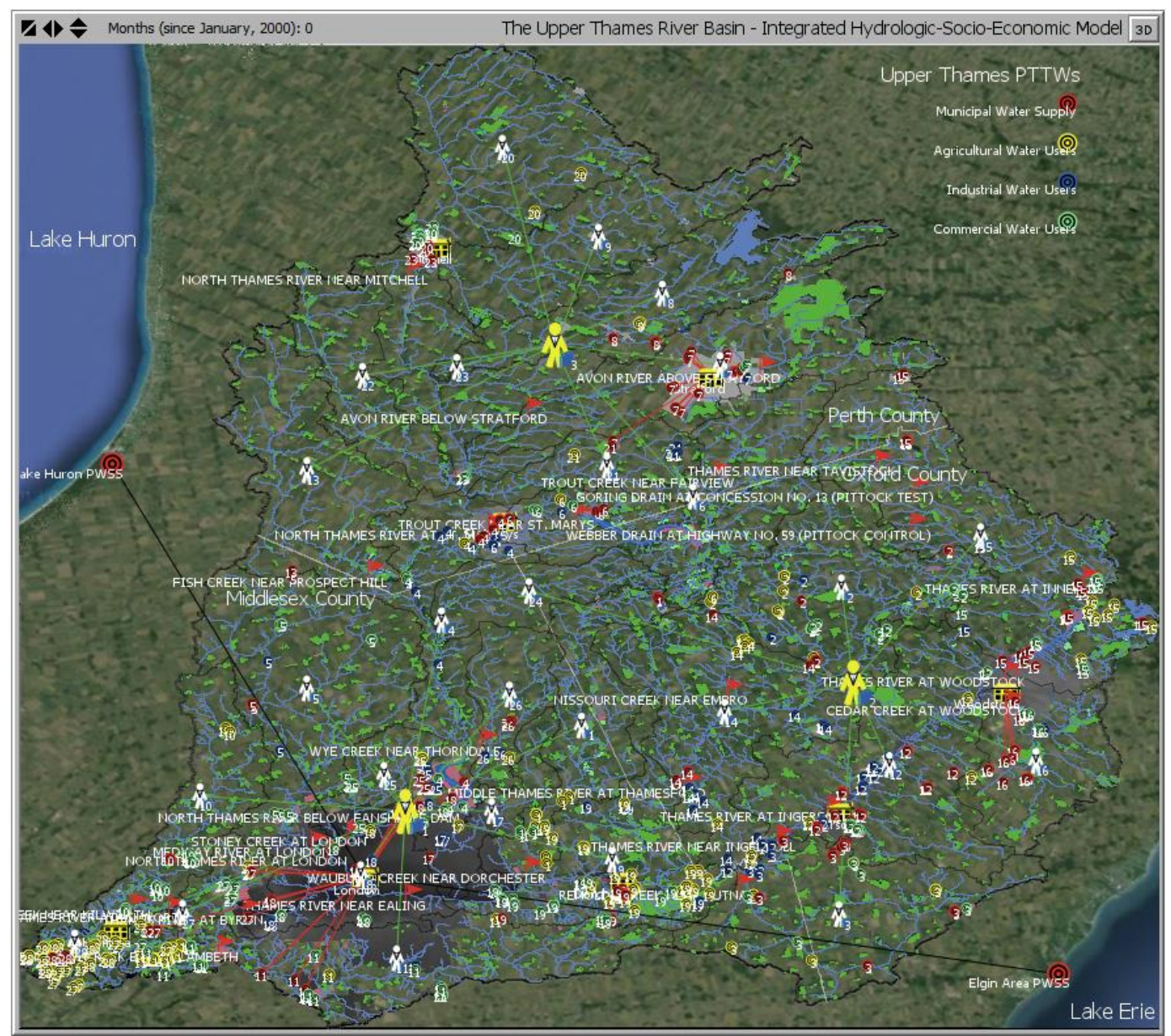

Figure 18: Main window of the model showing land-use map and active water users in each time step 


\subsubsection{Model entities}

The very first task in an agent-based modeling process is definition of entities that are essential for the overall system behavior. The list of things comprising the system can be endless, and, therefore, this first task helps to screen the most significant entities and a set of variables to describe each of them. On the basin scale, entities can be administrative and economic institutions, water users, urban centers, infrastructural objects, water treatment plants, etc. The Upper Thames River basin socio-economic agent-based model identifies following types of entities that influence and are influenced by the natural resources in the basin: registered water users (permits to take water database (PTTW) industrial, agricultural, commercial, municipal water supply), urban centers in the Upper Thames River Basin, primary water supply systems (PWSS), and decision makers on the sub-basin level and on the municipal (county) level, Figure 19.

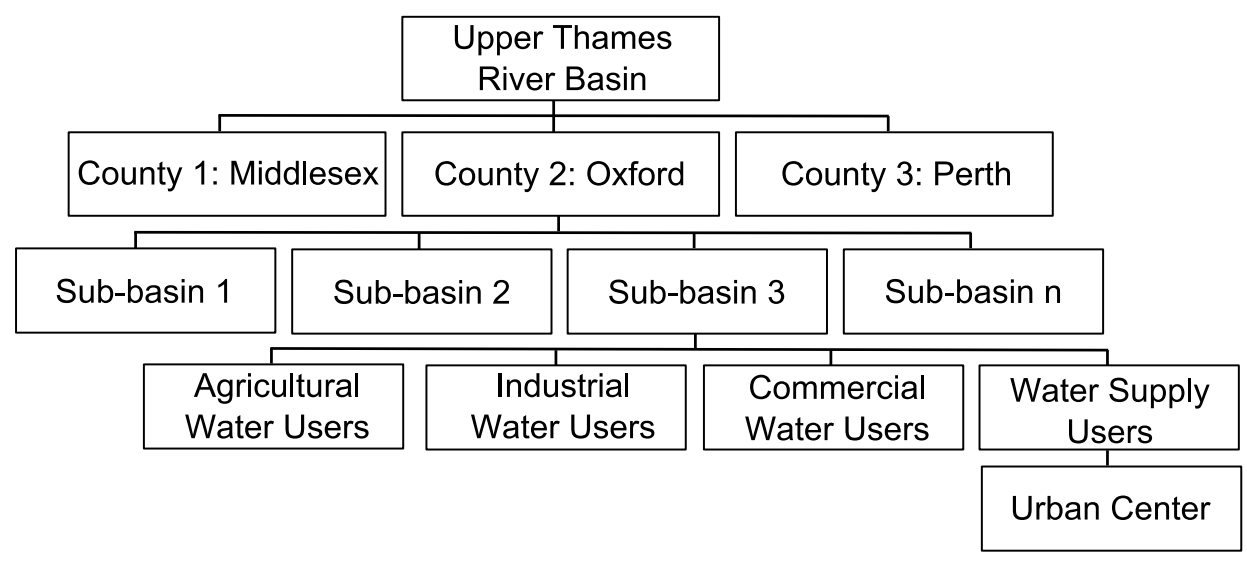

Figure 19: Recognized system actors by the socio-economic model

Each entity presents a respective collection of heterogenic members with a set of common attributes. In Netlogo modeling environment they are called Breeds. The model user can modify individual parameters through relevant databases or through a set of global parameters available in the main window. City agents (breed name in Netlogo: city-agents) group 8 major urban centers in the Upper Thames River basin (London, Ingersoll, St. Marys, Mitchell, Dorchester, Komoka, Woodstock, and Stratford). Each urban center contains a set of variables that define their individual demographic submodels and resulting water demand. Particular parameters of demographic sub-model 
depend on the capacity of water resources that are used for municipal water supply. Permit to take water (PTTW) database is used to characterize the individual users in the Upper Thames River basin. This database contains the individual permit holders that take more than 50,000 liters per day. This model presents the four most dominant types of water users in the UTRB: industrial users (industrial-pttws), agricultural users (agricultural-pttws), commercial users (commercial-pttws) and municipal water sources (water-supply-pttws). This model also includes two major sources of drinking water for municipal supply, the Lake Huron and the Elgin Area Primary Water Supply Systems (primary-wsss).

The Upper Thames River basin study area covers portions of 3 counties (Middlesex, Oxford, and Perth). Entities imported from the database represent responsible decision makers on the county level (municipal-managers). Also, 28 sub-basin managers represent 28 sub-basins in the case study (subbasin-managers). Both administrative agents on respective scales account current water consumption on one side, and water resources replenishment on the other.

All entities presented by the model include the exact spatial location and a number of other specific variables, such as maximum water demands or maximum capacities, and that information is imported from the shapefile database detailed in the previous section. Detailed description of variables is given in the following section. This model also includes a set of specific agents - series of undirected links, to describe data exchange corridors between different agents. Individual water users are linked with appropriate sub-basin managers according to their specific spatial location (agriculture-to-ws-links, industrial-to-ws-links, commercial-to-ws-links, watersupply-to-ws-links). The urban centers in the basin are linked with their respective water sources for municipal supply (city-to-watersource-links), while some of them are connected to the primary water supplying network.

Finally, for updating the current water balances on county level, all sub-basin managers are connected with the managers on the county level (subbasin-to-municipal-links). However, since database does not contain information on actual water withdrawals, the 
maximum permitted rates have been used for calculations (Thames - Sydenham and Region Drinking Water Source Protection, 2014). Since all water users contain particular location of water intakes, PTTW database is created in a form of a shapefile. Detailed description of the PTTW is given in the previous chapter.

\subsubsection{Model temporal and spatial scales}

According to the basic principles of integrated water resources management, a natural physical and operational unit for managing water resources is a river basin. Therefore, the spatial scale of developed model is a river basin, including all sub-basins and all administrative units such as counties. Total environment of the model contains $870 \times 752$ $=654,240$ patches. However, the spatially explicit model consists of 381,979 patches that represent just the area within the basin. One patch covers area of 0.009 ha. In this model, world wrapping is turned off since all agents are static. Each patch contains a set of variables (detailed in Variables section) that define the current land use, and belongs to one county and sub-basin. Time horizon of the simulation is 20 years. The simulation starts with data initialization for January 1, 2000 and ends with December 31, 2019 and it is executed on a monthly time step, with 240 time steps in total.

\subsubsection{Model variables}

For the illustration purposes, this section lists all variables for two types of entities represented by the model - the Upper Thames RB urban centers and industrial water users, Tables 11 and 12. Particular variables are read from the database (such as spatial location or annual water demand), and then used for further calculations according the

procedures described in the sub-models section. The list of remaining variables for all other entities is given in Appendix C. Figures 21 and 22 show individual lists of variables as seen in the main model window of Netlogo modeling platform. 
Table 11: Declared variables for the urban center entities

\begin{tabular}{cl}
\hline \multicolumn{2}{c}{ Upper Thames Urban Centers (city-agents-own) } \\
\hline city-id & Holds a unique ID number for eight major urban centers in \\
city-name & the UTRB \\
city-population & Name \\
birth-rate & Defrent population \\
max-birth-rate & Defines maximum birthrates \\
min-birth-rate & Defines maximum birthrates \\
death-rate & Defines death rates \\
city-monthly-water-demand & Monthly water demand based \\
capacity-of-my-water-sources & Capacity of water sources (surface and groundwater) \\
attractiveness-coefficient & Parameter calculated based on the water demand and water \\
& source capacities \\
\hline
\end{tabular}

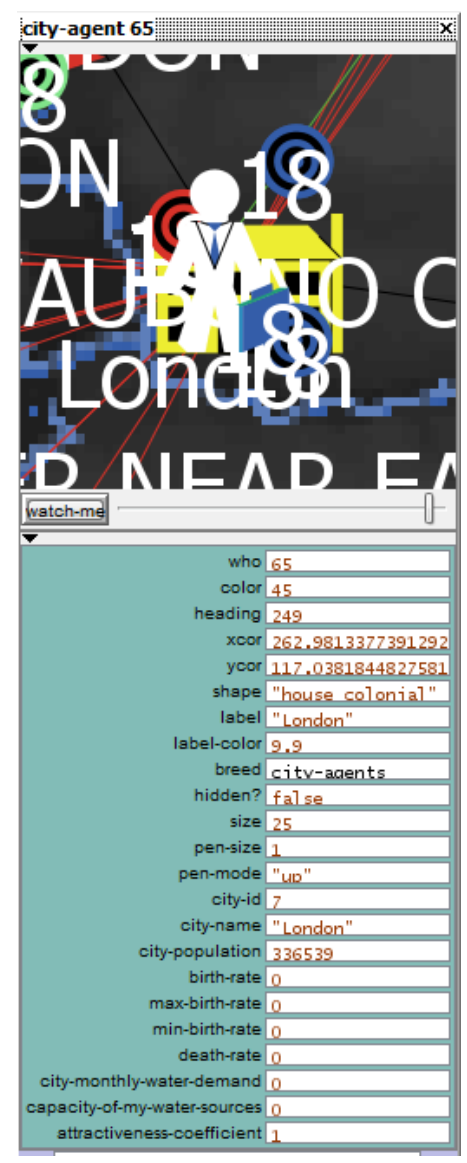

Figure 20: List of variables of an agent representing an urban center 
Table 12: Declared variables for the industrial water users

\begin{tabular}{|c|c|}
\hline \multicolumn{2}{|c|}{ Industrial Users (industrial-pttws-own) } \\
\hline sh id & Creates a link between an Industrial Water User and \\
\hline SD-1U & Responsible Manager on sub-basin level \\
\hline in-user-id & Unique industrial user ID \\
\hline in-specific-purpose & Industrial specific purpose \\
\hline in-issue-date & The date when the license is issued [Date Format] \\
\hline in-expiry-date & The date when the license expires [Date Format] \\
\hline in-issue-ticks & The date when the license is issued [Netlogo Format] \\
\hline in-expiry-ticks & The date when the license expires [Netlogo Format] \\
\hline in-max-litres-per-day & Maximum allowed liters per day to be taken [liters/day] \\
\hline in-days-per-year & $\begin{array}{l}\text { Maximum number of days per year allowed for taking } \\
\text { [days] }\end{array}$ \\
\hline in-total-annual-demand & Total demand per year $\left[\mathrm{m}^{3} /\right.$ year $]$ \\
\hline in-monthly-demand & Actual monthly demand $\left[\mathrm{m}^{3} /\right.$ month $]$ \\
\hline industrial-seasonal-variation-list & Seasonal industrial demand variation list [monthly] \\
\hline in-blue-footprint & Footprint per unit value added Blue Water $\left[\mathrm{m}^{3} / 1000 \$\right]$ \\
\hline in-grey-footprint & Footprint per unit value added Grey Water $\left[\mathrm{m}^{3} / 1000 \$\right]$ \\
\hline in-total-water-footprint & Total industrial water footprint per $1000 \$\left[\mathrm{~m}^{3} / 1000 \$\right]$ \\
\hline in-gross-economic-revenue & Gross economic revenue based on the water footprint [\$] \\
\hline in-water-use-costs & $\begin{array}{l}\text { Economic costs of water utilization based on the water } \\
\text { price for industry }[\$]\end{array}$ \\
\hline in-net-economic-revenue & Net economic revenue $[\$]$ \\
\hline active? & Defines if an agent is active in current time step \\
\hline
\end{tabular}




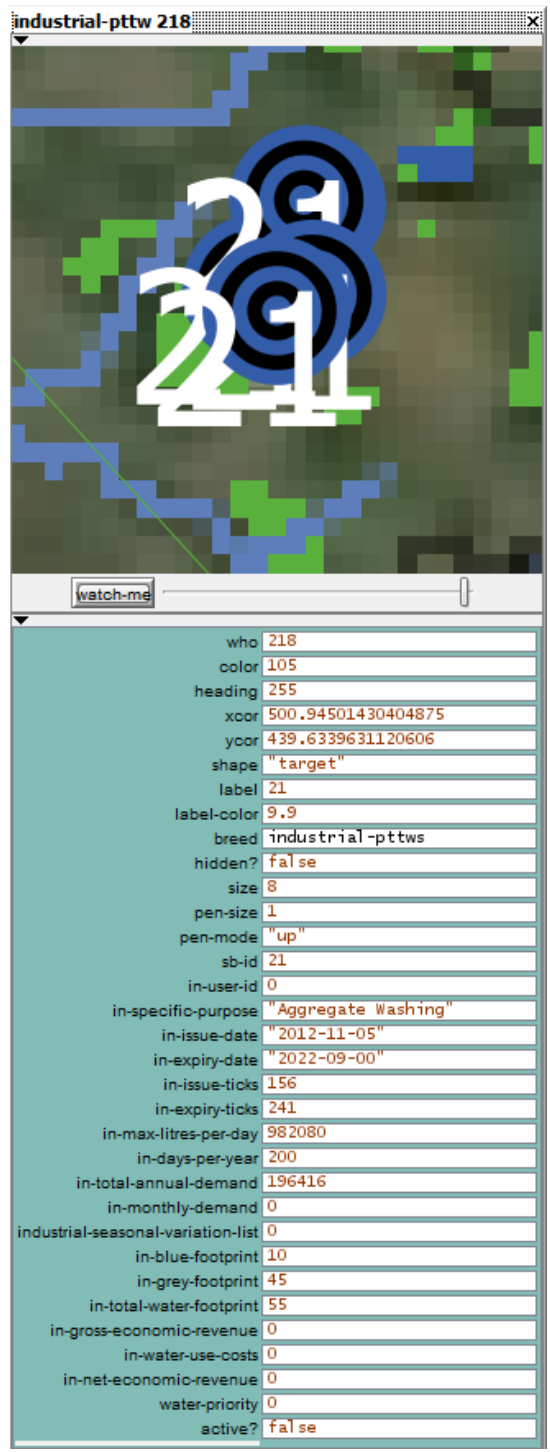

Figure 21: List of variables of an agent representing an industrial water user 


\subsubsection{Design concepts}

\section{Emergent behavior}

This model observes the emergent hydrologic and socio-economic system performance as a result of agent behavior that is directly controlled by the system user.

\section{Observation}

A number of monitors and plots are used to display the model hydrologic and socioeconomic outputs. First three plots show the accumulative water balance for selected three sub-basins (via appropriate choosers). Other plots present the information about surface river flows at three gauging stations (Byron, Ingersoll, and St. Marys), groundwater recharge rates and present water balance for all three counties, the current land use patterns in the basin, industrial and agricultural economic activities, and monthly water demand per sector.

\section{Adaptation}

The model user observes the current conditions of the system by analyzing the number of system outputs. In this way, the user is able to modify a number of parameters and thus directly adapt the behavior of agents. As a result, the user tries to find the optimal policy to ensure the long-term system stability by balancing the needs of socio-economic and hydrologic sub-systems.

\section{Sensing}

Links presented by the model symbolize the two way corridors for exchanging the information. All water users are linked to appropriate sub-basin managers depending on their spatial location. They send information through links about their current water demand, while the sub-basin managers calculate the current and cumulative water balance. On the other hand, sub-basin managers are linked with the agents representing managers on county level. Similarly to the previous case, the county level managers compute the current water balance in every time step. Agents that represent urban centers 
are linked to their sources of water (water-supply-pttws), and, in each time step, they compare the current municipal demand and the capacity of active sources.

\section{Scheduling}

This model contains a number of sub-models that represent particular hydrologic and socio-economic processes. Their order of execution is given in Figure 27, while each submodel is discussed in details in sub-models section.

\subsubsection{Initialization}

Before the agent-based model simulation is executed, all defined entities must be imported to the map, while their variables need to be initialized. Button "Model Setup", Figure 22, uses Netlogo's GIS extension and imports all required information from previously prepared databases.

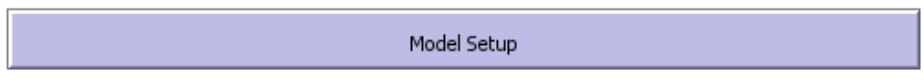

Figure 22: Model Setup button for initialization

The Upper Thames socio-economic model is spatially explicit, and, therefore, all imported agents contain spatial coordinates in addition to all other individual information. Names and details of initialization commands executed by the "Model Setup" button are given in Table 13.

Table 13: Initialization commands executed by the Model Setup button

\begin{tabular}{|c|c|}
\hline industrial-water-users & $\begin{array}{l}\text { Initiates Industrial Water Users based on PTTW database [ } \\
\text { Vector ] }\end{array}$ \\
\hline $\begin{array}{l}\text { agricultural-water- } \\
\text { users }\end{array}$ & $\begin{array}{l}\text { Initiates Agricultural Water Users based on PTTW database [ } \\
\text { Vector ] }\end{array}$ \\
\hline $\begin{array}{l}\text { commercial-water- } \\
\quad \text { users }\end{array}$ & $\begin{array}{l}\text { Initiates Commercial Water Users based on PTTW database [ } \\
\text { Vector ] }\end{array}$ \\
\hline $\begin{array}{l}\text { watersupply-water- } \\
\text { users }\end{array}$ & $\begin{array}{l}\text { Initiates municipal source of water supply based on PTTW } \\
\text { database [ Vector ] }\end{array}$ \\
\hline
\end{tabular}


primary- Initiates two primary water supplying systems [ Vector ]

watersupplying

landuse-map Initiates the land use map as of year 2000 [ Raster ]

municipalities Initiates the map of three municipalities [ Raster ]

subbasins Initiates the map of 28 sub-basins [ Raster ]

\subsubsection{Model switches}

Model switches allow a model user to choose what particular information will be presented in the main simulation window. Figure 23 shows the model switches presented by the model, while all switches are detailed in the Table 14.
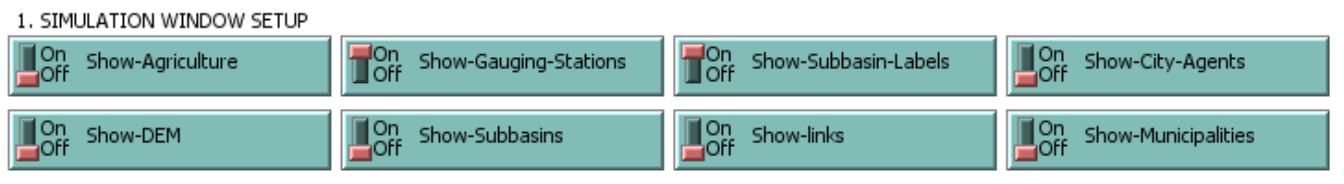

Figure 23: Model switches

Table 14: Model switches and their description

\begin{tabular}{cl}
\hline Switch Name & Description \\
\hline Show-Agriculture & Shows different types of agricultural activities \\
Show-DEM & Shows Digital Elevation Model in the main window \\
Show-Gauging-Stations & Shows Flow Gauging stations in the basin \\
Show-Subbasin-Labels & Shows the sub-basin labels \\
Show-City-Agents & Shows 8 urban centers \\
Show-Subbasins & Shows Sub-basin raster in the main window \\
Show-Links & Shows links between agents \\
Show-Municipalities & Shows Municipalities
\end{tabular}




\subsubsection{Model sliders}

Model sliders allow manual selection of a number of model parameters. Set of appropriate model parameters are used to define simulation scenarios. Detailed description of scenarios used to simulate different socio-economic and climate conditions in the basin is given in Chapter 4. The detailed description is followed by lists of parameters and their specific values that are used to describe each scenario. Figure 24 shows model sliders in the main window, while Table 15 presents the ranging values of each model slider.

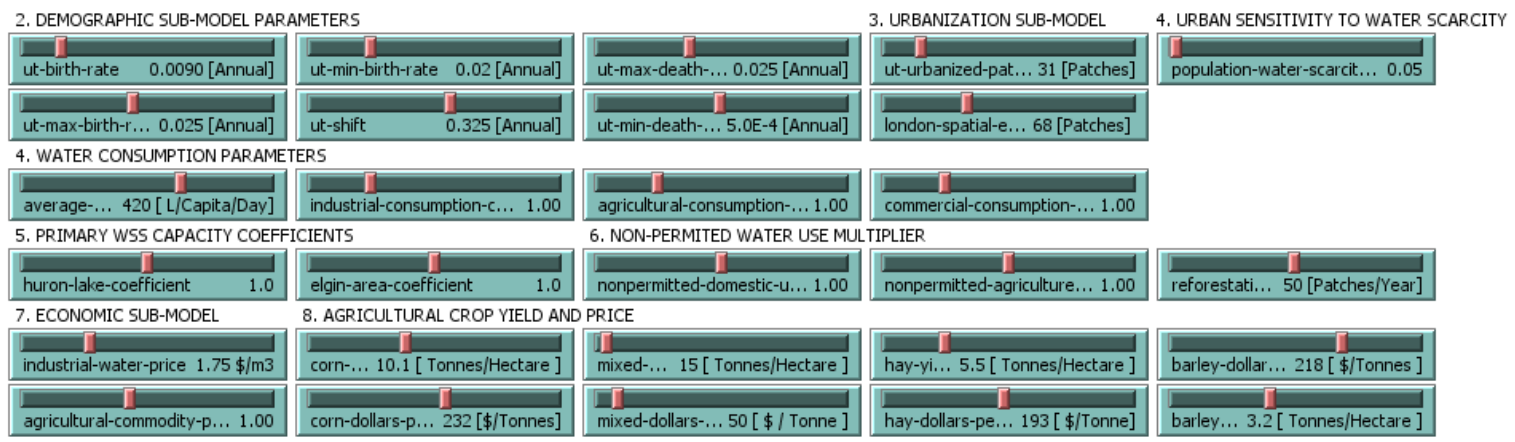

Figure 24: Model sliders used to parameterize the model

Table 15: List of model sliders that are used to define the model parameters

\begin{tabular}{|c|c|c|c|c|c|}
\hline Slider Name & Minimum & Increment & Maximum & $\begin{array}{l}\text { Initial } \\
\text { Value }\end{array}$ & Comments \\
\hline ut-birth-rate & 0.001 & 0.001 & 0.05 & 0.0090 & Current birth rate \\
\hline ut-max-birth-rate & 0.005 & 0.005 & 0.05 & 0.025 & Maximum birth rate \\
\hline ut-min-birth-rate & 0.01 & 0.02 & 0.05 & 0.02 & Minimum birth rate \\
\hline ut-shift & 0.1 & 0.005 & 0.5 & 0.325 & $\begin{array}{l}\text { Population shift } \\
\text { coefficient }\end{array}$ \\
\hline ut-max-death-rate & 0.01 & 0.005 & 0.05 & 0.025 & Maximum death rate \\
\hline ut-min-death-rate & 0.00001 & 0.0001 & 0.001 & $5.0 \mathrm{E}-4$ & Minimum death rate \\
\hline $\begin{array}{l}\text { average-per-capita- } \\
\text { per-day-water- } \\
\text { consumption }\end{array}$ & 200 & 25 & 550 & 420 & $\begin{array}{l}\text { Average per capita per } \\
\text { day water consumption } \\
\text { [L/day/capita] }\end{array}$ \\
\hline $\begin{array}{l}\text { industrial- } \\
\text { consumption- } \\
\text { coefficient }\end{array}$ & 0 & 0.25 & 4 & 1 & $\begin{array}{l}\text { Industrial consumption } \\
\text { multiplication } \\
\text { coefficient }\end{array}$ \\
\hline $\begin{array}{l}\text { agricultural- } \\
\text { consumption- } \\
\text { coefficient }\end{array}$ & 0 & 0.25 & 4 & 1 & $\begin{array}{l}\text { Agricultural } \\
\text { consumption } \\
\text { multiplication } \\
\text { coefficient }\end{array}$ \\
\hline commercial- & 0 & 0.25 & 4 & 1 & Commercial \\
\hline
\end{tabular}




\begin{tabular}{|c|c|c|c|c|c|}
\hline $\begin{array}{l}\text { consumption- } \\
\text { coefficient }\end{array}$ & & & & & $\begin{array}{l}\text { consumption } \\
\text { multiplication } \\
\text { coefficient }\end{array}$ \\
\hline $\begin{array}{l}\text { huron-lake- } \\
\text { coefficient }\end{array}$ & 0 & 0.1 & 2 & 1 & $\begin{array}{l}\text { Huron Lake capacity } \\
\text { operation multiplier }\end{array}$ \\
\hline elgin-area-coefficient & 0 & 0.1 & 2 & 1 & $\begin{array}{l}\text { Elgin Area capacity } \\
\text { operation multiplier }\end{array}$ \\
\hline $\begin{array}{l}\text { nonpermitted- } \\
\text { domestic-use- } \\
\text { multiplier }\end{array}$ & 0 & 0.05 & 2 & 1 & $\begin{array}{l}\text { Non-permitted use } \\
\text { multiplier for domestic } \\
\text { purposes }\end{array}$ \\
\hline $\begin{array}{l}\text { nonpermitted- } \\
\text { agriculture-use- } \\
\text { multiplier }\end{array}$ & 0 & 0.05 & 2 & 1 & $\begin{array}{l}\text { Non-permitted use } \\
\text { multiplier for agriculture } \\
\text { purposes }\end{array}$ \\
\hline $\begin{array}{l}\text { population-water- } \\
\text { scarcity-sensitivity }\end{array}$ & 0.05 & 0.05 & 1 & 0.05 & $\begin{array}{l}\text { Determines the } \\
\text { municipal sensitivity to } \\
\text { the water scarcity }\end{array}$ \\
\hline industrial-water-price & 0.5 & 0.25 & 5 & 1.5 & $\begin{array}{l}\text { Determined price of } \\
\text { water industrial users }\end{array}$ \\
\hline $\begin{array}{c}\text { agricultural- } \\
\text { commodity-price- } \\
\text { variation }\end{array}$ & 0.25 & 0.25 & 2 & 1 & $\begin{array}{l}\text { Agricultural production } \\
\text { Price multiplier [ } \$ / \mathrm{kg} \text { ] }\end{array}$ \\
\hline $\begin{array}{l}\text { london-spatial- } \\
\text { expansion }\end{array}$ & 0 & 2 & 200 & 68 & $\begin{array}{l}\text { Number of patches } \\
\text { occupied by London in } \\
\text { each time step }\end{array}$ \\
\hline ut-urbanized-patches & 0 & 1 & 200 & 31 & $\begin{array}{l}\text { Number of urbanized } \\
\text { patches in UT per time } \\
\text { step }\end{array}$ \\
\hline reforestation-rate & 1 & 50 & 500 & 100 & $\begin{array}{l}\text { Number of patches for } \\
\text { reforestation in each } \\
\text { time step }\end{array}$ \\
\hline $\begin{array}{l}\text { corn-yield-tonnes- } \\
\text { per-hectare }\end{array}$ & 7 & 0.1 & 15 & 10.1 & $\begin{array}{l}\text { Corn yield per hectare }[\mathrm{t} \\
\text { / hectare }]\end{array}$ \\
\hline $\begin{array}{l}\text { corn-dollars-per- } \\
\text { tonnes }\end{array}$ & 150 & 5 & 300 & 232 & $\begin{array}{l}\text { Price of corn per tonne [ } \\
\$ / \text { tonne ] }\end{array}$ \\
\hline $\begin{array}{l}\text { mixed-yield-tonnes- } \\
\text { per-hectare }\end{array}$ & 2 & 5 & 250 & 15 & $\begin{array}{l}\text { Mixed systems yield per } \\
\text { hectare [ } \mathrm{t} / \text { hectare ] }\end{array}$ \\
\hline $\begin{array}{l}\text { mixed-dollars-per- } \\
\text { tonnes }\end{array}$ & 25 & 5 & 300 & 50 & $\begin{array}{l}\text { Price of corn per tonne [ } \\
\$ / \text { tonne ] }\end{array}$ \\
\hline $\begin{array}{l}\text { hay-yield-tonnes-per- } \\
\text { hectare }\end{array}$ & 4 & 0.5 & 10 & 5.5 & $\begin{array}{l}\text { Hay yield per hectare }[\mathrm{t} \\
\text { / hectare }]\end{array}$ \\
\hline $\begin{array}{l}\text { hay-dollars-per- } \\
\text { tonnes }\end{array}$ & 140 & 1 & 250 & 193 & $\begin{array}{l}\text { Price of hay per tonne [ } \\
\$ / \text { tonne ] }\end{array}$ \\
\hline $\begin{array}{l}\text { barley-dollars-per- } \\
\text { tonnes }\end{array}$ & 150 & 2 & 250 & 218 & $\begin{array}{l}\text { Barley yield per hectare } \\
\text { [ } \mathrm{t} / \text { hectare }]\end{array}$ \\
\hline $\begin{array}{l}\text { barley-yield-tonnes- } \\
\text { per-hectare }\end{array}$ & 2 & 0.2 & 5 & 3.2 & $\begin{array}{l}\text { Price of barley per tonne } \\
\text { [\$/tonne ] }\end{array}$ \\
\hline
\end{tabular}




\subsubsection{Model choosers}

Defined choosers allow the model user to select one of the parameters from the predefined lists. Figure 25 shows the model chooser presented in the model, while Table 16 gives additional information.

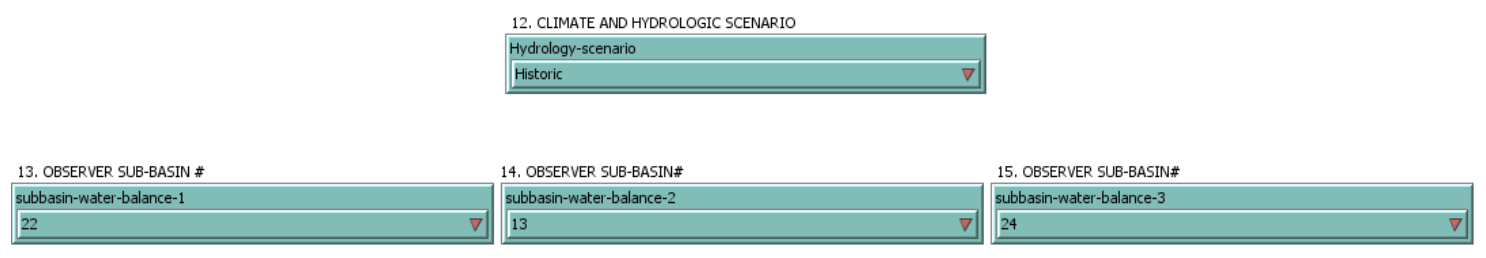

Figure 25: Model choosers

Table 16: List of model choosers and their description

\begin{tabular}{ccc}
\hline Chooser Name & Chooser Action & Chooser Options \\
\hline hydrology-scenario & Initiates one out of three hydrologic scenarios & Historical, Wet, Dry \\
subbasin-water-balance-1 & Shows results for selected sub-basin & $1-28$ \\
subbasin-water-balance-2 & Shows results for selected sub-basin & $1-28$ \\
subbasin-water-balance-3 & Shows results for selected sub-basin & $1-28$ \\
\hline
\end{tabular}

\subsubsection{Sub-models}

An agent-based model can be seen as a collection of sub-models that subsequently simulate the most important processes from the aspect of system performance. Submodels are designed, tested and analyzed independently before the final integration. In Netlogo, sub-models represent newly created procedures, and each procedure is described in the following section. Figure 26 shows all sub-models of integrated model in scheduled order of execution, while following section details each sub-model separately. 


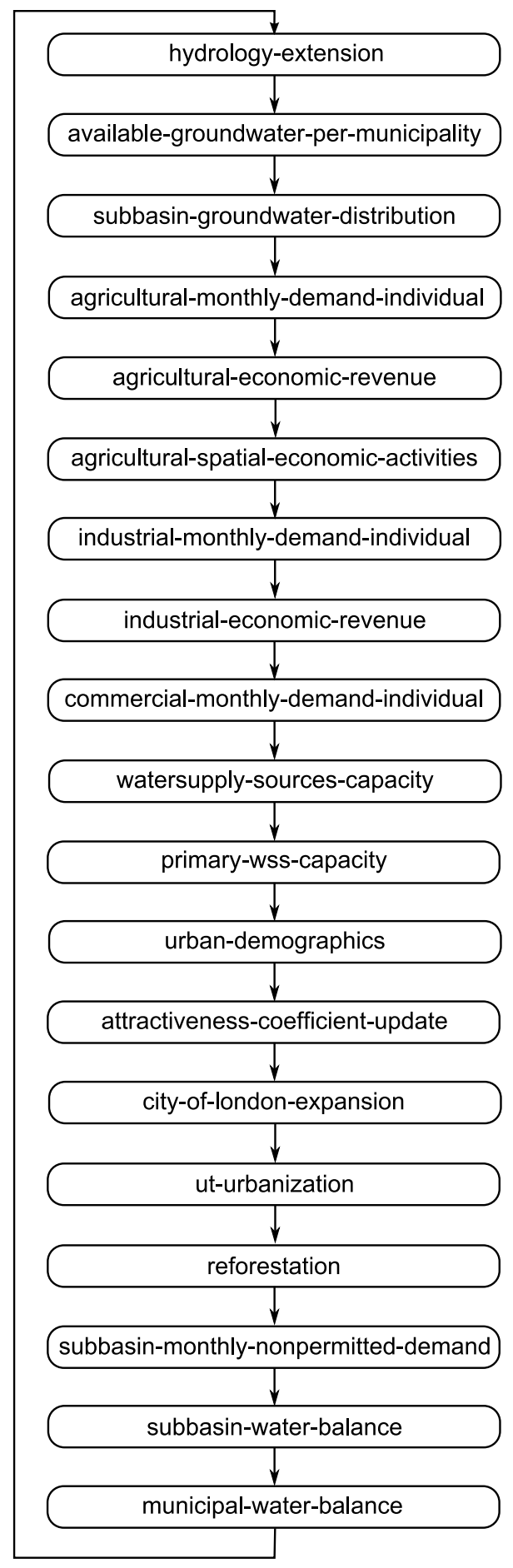

Figure 26: Sub-models and their order of execution 


\section{Sub-model 1: hydrology-extension}

In contrast to the traditional modeling approaches which describe processes separately and possibly on different spatial and temporal scales, the integrated Upper Thames River basin model (IHSEM-UTRB) couples the hydrologic and socio-economic system components via feedback links. Hydrologic-extension procedure is created as a new Netlogo's Extension to establish the direct data exchange link between spatially semidistributed hydrologic model and socio-economic agent-based model.

The semi-distributed hydrologic component delivers climate variables (precipitation and temperature), groundwater recharge rates and stream flows to the socio-economic component. Before it is executed, the hydrologic model reads the information that describes the current physical state of the system, and then runs on a six hour time step. Parameters "Fraction of Vegetated Land" (fraction-vegetated-land-middlesex-output, fraction-vegetated-land-oxford-output, fraction-vegetated-land-perth-output) and "Fraction of Paved Land" (fraction-paved-land-middlesex-output, fraction-paved-landoxford-output, fraction-paved-land-perth-output) are calculated for 3 counties to describe local physical conditions and then they are passed to the hydrologic model. For updated hydrologic parameters, hydrologic model calculates groundwater recharge for all three counties and surface flows for three flow gauging stations at six hour time step. At the end of each month, the obtained values are communicated to the socio-economic model, and this information is then transferred to designated agents. As described previously, a monthly time step is used to represent all significant socio-economic process in the system. At this point, the socio-economic model uses the hydrologic outputs to initiate and then executes all scheduled sub-models. Changed socio-economic conditions after one time step update the physical state of the hydrologic system.

Under the initial assumptions that physical conditions and hydrologic regimes are affected by the socio-economic conditions, the direct link between the two is established through a number of system parameters. At the start of the second month, the hydrologic model updates following parameters: potential evapotranspiration (PET), maximum surface storage (MSS) and maximum soil infiltration capacity (MSI). The level of change 
of hydrologic parameters comes from the land use sector represented in the socioeconomic model. Figure 27 defines the assumed relationship between the fraction of paved land and the effect of paved land on the surface storage capacity. The fraction of paved land represents urbanized areas in the basin divided by the total basin area. This relationship assumes that as the fraction of paved land increases, the surface storage capacity decreases causing higher excess runoff. Higher runoff directly reduces the amount of water that can infiltrate into the soil and lower the groundwater recharge levels.

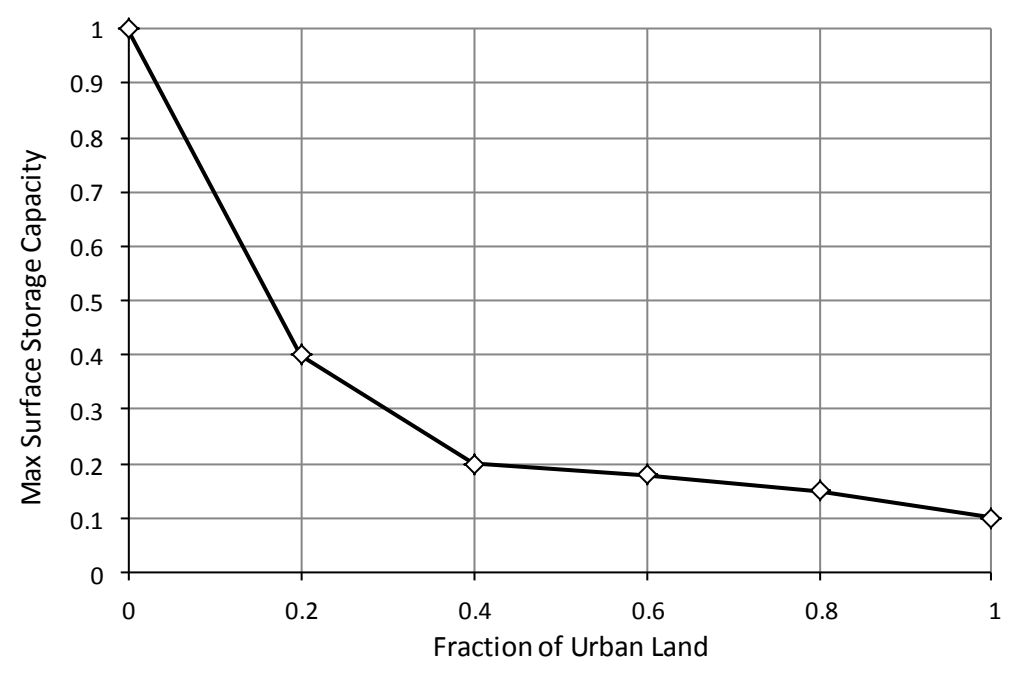

Figure 27: Maximum Surface Storage Effect look-up table

On other side, the fraction of vegetated cover is defined as a sum of forest and woodlands cover and agricultural land. Figure 28 shows the dependence between the fraction of vegetated cover and the maximum soil infiltration capacity. The infiltration capacity depends on a number of physical parameters, such as soil type, content of soil moisture, content of organic matter, vegetative cover, and season (Linsley et al., 1958). Since the soil porosity influence the flow through the media, the type of soil is the most important factor of infiltration capacity. Increased soil porosity increases the infiltration capacity. Also, vegetation increases the soil porosity and therefore, increases the infiltration capacity. Therefore, the maximum soil infiltration capacity increases as vegetative cover increases, and as the vegetative cover decreases, the infiltration capacity also decreases, further lowering the groundwater recharge rates and increasing the surface runoff. 


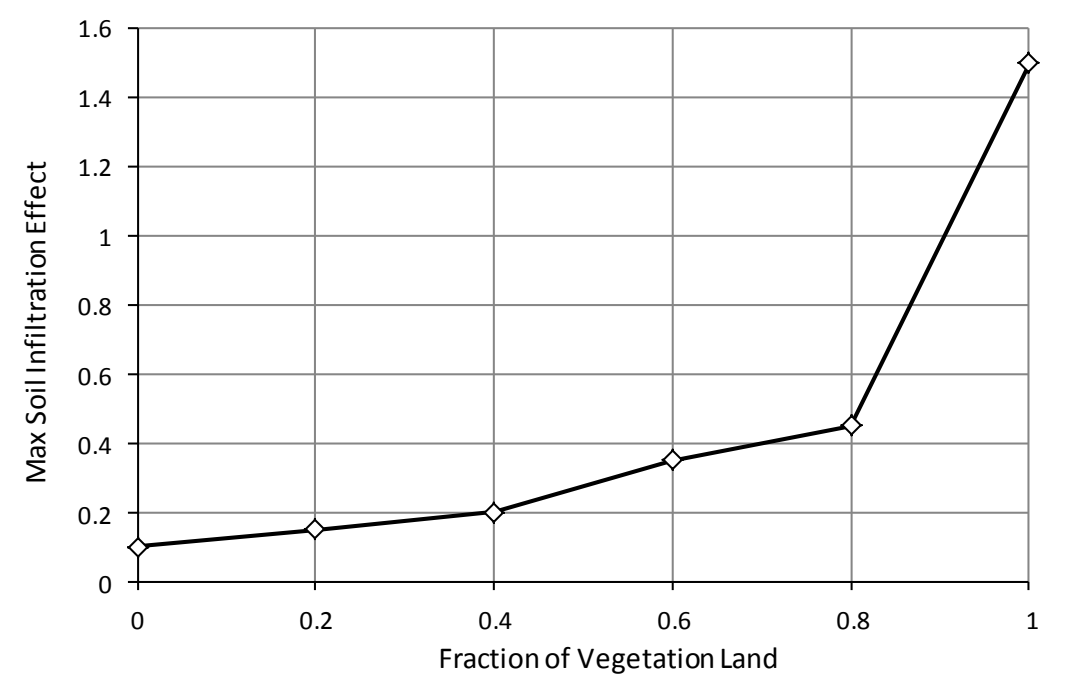

Figure 28: Maximum Soil Infiltration Effect look-up table

Figure 29 displays the relationship between the fraction of vegetated cover and potential evapotranspiration. Generally speaking, potential evapotranspiration represents a physical quantity estimated using empirical equations and primarily depends on climate factors. However, for the sake of this study, it is assumed that evapotranspiration strongly depends on vegetation cover since as vegetation cover increases, the total amount of water lost to the atmosphere from vegetation also increases (Brutsaert, 1982).

Netlogo's extension feature is used to create a communication and direct data exchange link between the two modeling environments. The extension combines an agent-based model developed in Netlogo and a hydrologic model developed in Java programming environment. Programming details and code snippets for the creation of extension are given in Appendix B. 


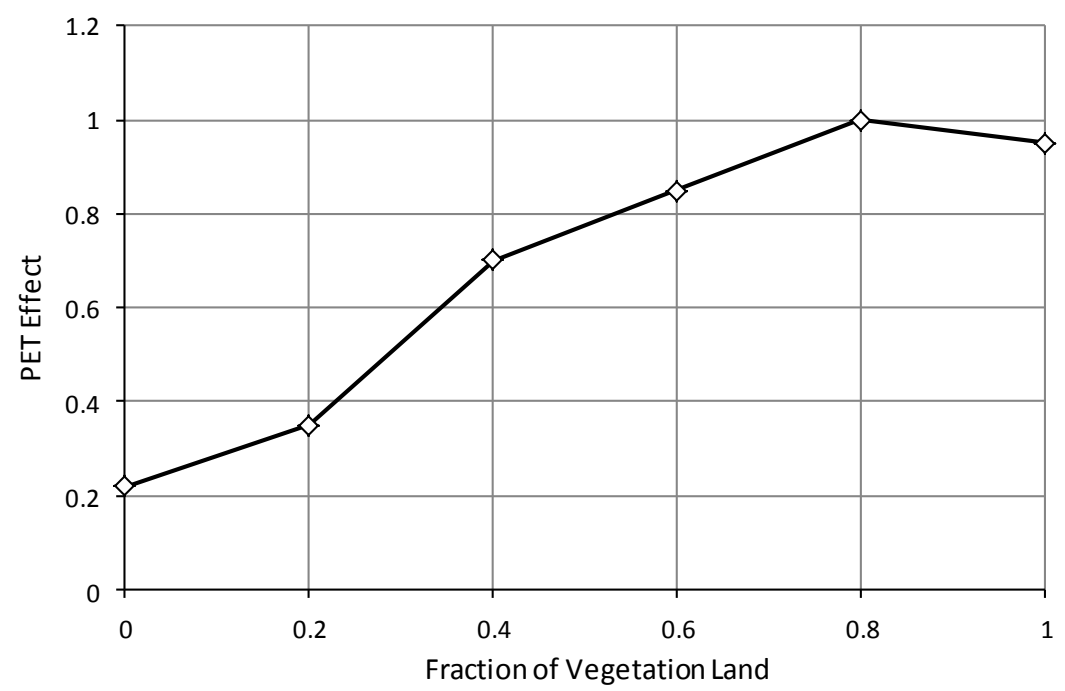

Figure 29: Potential Evapotranspiration look-up table

\section{Sub-model 2: available-groundwater-per-municipality}

The hydrology model calculates the ground water recharge rates for three counties (Middlesex, Oxford, and Perth) in the UTRB (municipal-groundwater-recharge-rate). These values are then passed to the agents that represent the managerial bodies on the county level (municipal-managers).

\section{Sub-model 3: sub-basin-groundwater distribution}

To obtain the ground water recharge rates (subbasin-groundwater-recharge) on the subbasin level, agents representing the sub-basin decision makers (sub-basin-managers) multiply the value of percentage of municipality they occupy (percentage-ofmunicipality-area) and groundwater recharge rate (municipal-groundwater-rechargerate) in the current time step from respective municipality.

\section{Sub-model 4: agricultural-monthly-demand-individual}

This procedure calculates the actual monthly demand (ag-monthly-demand) for all individual agricultural users. Agricultural water users can be active or inactive (active?) in every time step, depending on the date when the permit is issued (ag-issue-ticks) and 
when it expires (ag-expiry-ticks). Since the individual demand varies depending on the season and specific agricultural purpose, the maximum annual permitted value (ag-totalannual-demand), obtained from the PTTW database, is multiplied with a seasonal variation list coefficient (agricultural-seasonal-variation-list). This list uses averaged agricultural demand values for each month, Table 17, according to Statistics Canada (2010b):

Table 17: Seasonal variation of agricultural water demand

\begin{tabular}{|c|c|c|c|c|c|c|c|c|c|c|c|}
\hline \multicolumn{12}{|c|}{ agricultural-seasonal-variation-list } \\
\hline I & II & III & IV & $\mathrm{V}$ & VI & VII & VIII & IX & $\mathrm{X}$ & XI & XII \\
\hline 0 & 0 & 0 & 0.029 & 0.029 & 0.1229 & 0.4693 & 0.2979 & 0.0321 & 0.0321 & 0 & 0 \\
\hline
\end{tabular}

The model user is allowed to interactively modify the agricultural water demand via Agricultural Consumption Variation Coefficient (slider: agricultural-consumptioncoefficient), and observe the resulting system behavior.

\section{Sub-model 5: agricultural-economic-revenue}

This procedure calculates the individual gross economic revenue (ag-gross-economicrevenue) for all agricultural water users. This value is computed based on the consumed volume of water in the particular time step (ag-monthly-demand). In this case, it is assumed that the user utilizes the actual demanded amount of water. All agricultural water users have a unique value of the water footprint (ag-water-footprint) that represents the total volume of water used to produce the product. Typical agricultural activities have water footprints shown in Table 18, after Mekonnen and Hoekstra (2011a).

Calculated produced commodities (ag-produced-commodities) for agricultural agents depend on the amount of used water (ag-monthly-demand) and the individual water footprint (ag-water-footprint):

ag-produced-commodities $=$ ag-monthly-demand $/$ ag-water-footprint

ag-gross-economic-revenue $=$ ag-produced-commodities $*$ ag-unit-commodity-price 
Table 18: Different types of products and their water footprint

\begin{tabular}{cc}
\hline Type of product & liter/kg \\
\hline Sugar crops & 197 \\
Vegetables & 322 \\
Starchy roots & 387 \\
Fruits & 962 \\
Cereals & 1644 \\
Oil crops & 2364 \\
Pulses & 4055 \\
Nuts & 9063 \\
Milk & 1020 \\
Eggs & 1020 \\
Chicken meat & 4325 \\
Butter & 5553 \\
Pig meat & 5988 \\
Sheep/goat meat & 8763 \\
Bovine meat & 15415 \\
\hline
\end{tabular}

\section{Sub-model 6: agricultural-spatial-economic-activities}

This procedure is executed only once every year at the end of the agricultural season (seasonal-list-item-counter $=9$ ). This procedure calculates the economic revenues (yieldrevenue) based on the total area under particular crop (Corn, Hay System, Continuous Row Crop - Barley, Mixed Systems) and given economic parameters defined by the model user, such as yield per hectare and price per one tonne.

\section{Corn}

For defined corn yield per hectare (corn-yield-tonnes-per-hectare) and price per tonnes (corn-dollars-per-tonnes), the total economic revenue from corn:

Hay system

For defined hay yield per hectare (hay-yield-tonnes-per-hectare) and price per tonnes (hay-dollars-per-tonnes), the total economic revenue from hay: 
hay-yield-revenue $=$ hay-fields-hectares $*$ hay-yield-tonnes-per-hectare $*$ hay-dollars-per-tonnes

Continuous row crop - Barley

For defined barley yield per hectare (barley-yield-tonnes-per-hectare) and price per tonnes (barley-dollars-per-tonnes), the total economic revenue from barley:

barley-yield-revenue $=$ barley-fields-hectares $*$ barley-yield-tonnes-per-hectare $*$ barley-dollars-per- $(44)$ tonnes

Mixed systems

For defined mixed system yield per hectare (mixed-yield-tonnes-per-hectare) and price per tonnes (mixed-dollars-per-tonnes), the total economic revenue from mixed systems:

mixed-yield-revenue $=$ mixed-fields-hectares $*$ mixed-yield-tonnes-per-hectare $*$ mixed-dollars-per- $(45)$ tonnes

\section{Sub-model 7: industrial-monthly-demand-individual}

This sub-model calculates the monthly industrial water demand (in-monthly-demand) for each industrial agent, based on the maximum annual permitted takings (in-total-annualdemand). Since the industrial demand can vary depending on the season, the maximum annual permitted value (in-total-annual-demand), obtained from the PTTW database, is multiplied with a monthly coefficient from seasonal variation list (industrial-seasonalvariation-list). In this case, seasonal variation list assumes that the water demand is equal throughout the year, assigning the coefficient of 0.0833 to all months, Table 19.

Table 19: Seasonal water demand variation list for industrial users

\begin{tabular}{|c|c|c|c|c|c|c|c|c|c|c|c|}
\hline \multicolumn{12}{|c|}{ industrial-seasonal-variation-list } \\
\hline I & II & III & IV & $\mathrm{V}$ & VI & VII & VIII & IX & X & XI & XII \\
\hline 0.0833 & 0.0833 & 0.0833 & 0.0833 & 0.0833 & 0.0833 & 0.0833 & 0.0833 & 0.0833 & 0.0833 & 0.0833 & 0.083 \\
\hline
\end{tabular}

The model user can interactively change the total Industrial Consumption Variation Coefficient (slider: industrial-consumption-coefficient) to represent the increase or decrease in industrial water demand. Depending on the date when the permit to take 
water is issued (in-issue-ticks) and when it expires (in-expiry-ticks), industrial water users can be active or inactive (active? = true or false) in every time step.

\section{Sub-model 8: industrial-economic-revenue}

This sub-model calculates the economic activity of industrial water users based on the individual monthly water demand (in-monthly-demand) and the water footprint (in-totalwater-footprint). Water footprint represents the measure of human appropriation of freshwater resources and represents the water volumes consumed (incorporated into a product) or polluted per unit of time. The total water footprint has three components: green, blue and grey. The blue water footprint represents the consumption of blue water resources (surface and ground), while the green water footprint refers to the volume of green water - rainwater. Finally, the grey water footprint indicates the degree of freshwater pollution and it represents the volume of freshwater that is required to assimilate the load of pollutants based on existing ambient water quality standards. Table 20 shows the average water footprint of industrial products per unit of industrial value added (1996-2005), Mekonnen and Hoekstra, (2011b).

Table 20: Canadian industrial water footprint $\left(\mathrm{m}^{3} / 1000 \$\right)$

\begin{tabular}{ccccc}
\hline \multirow{2}{*}{$\begin{array}{c}\text { FAOSTAT } \\
\text { code }\end{array}$} & Country & \multicolumn{3}{c}{$\begin{array}{c}\text { Average water footprint per } \\
\text { unit value added }\left(\mathrm{m}^{3} / 1000 \text { US }\right.\end{array}$} \\
\cline { 3 - 5 } & & Blue & Grey & Total \\
\hline 33 & Canada & 7.03 & 37.8 & 44.8 \\
\hline
\end{tabular}

Gross economic revenue:

in-gross-economic-revenue $=$ in-monthly-demand $* 1000 /$ in-total-water-footprint

Costs of water utilization based on the user defined prices of water for industry:

$$
\text { in-water-use-costs }=\text { in-monthly-demand } * \text { industrial-water-price }
$$

Finally, the individual net economic revenue for industrial water users:

$$
\text { in-net-economic-revenue }=\text { in-gross-economic-revenue } \text { - in-water-use-costs }
$$




\section{Sub-model 9: commercial-monthly-demand-individual}

In this procedure, the actual monthly demand (co-monthly-demand) for individual commercial users is calculated. Since the demand varies depending on the season (seasonal-list-item-counter), the maximum annual permitted value (co-total-annualdemand), obtained from the PTTW database, is multiplied with values presented as a seasonal variation list (commercial-seasonal-variation-list) in Table 21.

Table 21: Seasonal water demand variation list for commercial water users

\begin{tabular}{|c|c|c|c|c|c|c|c|c|c|c|c|}
\hline \multicolumn{12}{|c|}{ commercial-seasonal-variation-list } \\
\hline I & II & III & IV & $\mathrm{V}$ & VI & VII & VIII & IX & $\mathrm{X}$ & XI & XII \\
\hline 0 & 0 & 0 & 0.029 & 0.029 & 0.1229 & 0.4693 & 0.2979 & 0.032 & 0.0321 & 0 & 0 \\
\hline
\end{tabular}

The model user is able to manually influence the commercial water demand via Commercial Consumption Variation Coefficient (slider: commercial-consumptioncoefficient). Commercial water users can be active or inactive (active $?=$ true or false) in every time step, depending on the date when the permit is issued (co-issue-ticks) and when it expires (co-expiry-ticks).

$$
\begin{gathered}
\text { co-monthly-demand }=\text { co-total-annual-demand } * \text { item }(\text { seasonal-list-item-counter }-1) \text { commercial- } \\
\text { seasonal-variation-list } * \text { commercial-consumption-coefficient }
\end{gathered}
$$

\section{Sub-model 10: watersupply-sources-capacity}

This sub-model firstly determines if a water source agent is active (active $?=$ true or false) in the current time step based on the date when the permit is issued (ws-issue-ticks) and when it expires (ws-expiry-ticks). If the agent is not active in this time step, then its capacity is 0 (ws-max-monthly-capacity $=0$ ). In contrast, if the agent is active, the actual monthly capacity (ws-max-monthly-capacity) is calculated as a function of annual capacity (ws-max-annual-capacity) and seasonal variation coefficient stored in the seasonal variation list (water-supply-seasonal-variation-list), Table 22. 
Table 22: Seasonal water demand variation list for municipal water users

\begin{tabular}{cccccccccccc}
\hline \multicolumn{11}{c}{ water-supply-seasonal-variation-list } \\
\hline I & II & III & IV & V & VI & VII & VIII & IX & X & XI & XII \\
0.83 & 0.83 & 0.83 & 0.83 & 0.83 & 0.83 & 0.83 & 0.83 & 0.83 & 0.83 & 0.83 & 0.83 \\
\hline
\end{tabular}

Individual monthly capacity of water:

ws-max-monthly-capacity = ws-max-annual-capacity $*$ item (seasonal-list-item-counter - 1$)$ watersupply-seasonal-variation-list

\section{Sub-model 11: primary-wss-capacity}

This procedure calculates the individual capacities of both water supply systems (Elgin Area PWSS and Huron Lake PWSS). Annual capacity is obtained from the shapefile, while the actual monthly capacity is calculated by multiplying that value with appropriate monthly item at the seasonal variation list (primary-water-system-seasonal-variationlist), Table 23.

Table 23: Primary water supply systems variation list

\begin{tabular}{cccccccccccc}
\hline \multicolumn{11}{c}{ primary-water-system-seasonal-variation-list } \\
\hline I & II & III & IV & V & VI & VII & VIII & IX & X & XI & XII \\
0.042 & 0.042 & 0.83 & 0.83 & 0.83 & 0.125 & 0.125 & 0.125 & 0.83 & 0.83 & 0.042 & 0.083 \\
\hline
\end{tabular}

Monthly capacity also is a function of a coefficient that is set by the model user via sliders (huron-lake-coefficient and elgin-area-coefficient). These coefficients determine the potential variation in capacity and the effects that it remains on the urban population.

Huron Lake PWSS monthly capacity:

ws-max-monthly-capacity $=$ pwss-total-annual-capacity $*$ item $($ seasonal-list-item-counter

- 1) primary-water-system-seasonal-variation-list * huron-lake-coefficient

Elgin Area PWSS monthly capacity:

ws-max-monthly-capacity $=$ pwss-total-annual-capacity $*$ item (seasonal-list-item-counter

- 1) primary-water-system-seasonal-variation-list * elgin-area-coefficient 


\section{Sub-model 12: urban-demographics}

This sub-model represents the population dynamics (city-population) for 8 urban centers in the UTRB. The model user selects from the slider parameters on annual level (birthrate, max-birth-rate, min-birth-rate, shift, max-death-rate, min-death-rate, and deathrate), while these parameters are then converted to the monthly values in the model code.

$$
\begin{aligned}
& \text { pop-change ((birth-rate - death-rate) } * \text { city-population } * \text { attractiveness-coefficient }) \\
& \text { city-population }=\text { int }(\text { city-population }+ \text { pop-change })
\end{aligned}
$$

Also, this sub-model calculates the actual monthly municipal water demand (citymonthly-water-demand) based on the population (city-population) and the average per capita daily water consumption defined by the model user (average-per-capita-per-daywater-consumption) via slider.

$$
\begin{aligned}
& \text { city-annual-water-demand city-population } * 365 * \text { average-per-capita-per-day-water-consumption } \\
& \text { city-monthly-water-demand ( city-annual-water-demand / } 12 \text { ) / } 1000
\end{aligned}
$$

\section{Sub-model 13: attractiveness-coefficient-update}

Based on the current monthly municipal water demand, this procedure calculates the attractiveness coefficient (attractiveness-coefficient) for each urban center in the system by comparing the demand and actual capacity of water sources (Primary Water Supply System and Municipal PTTW). Parameter that defines the municipal resilience to water scarcity is set by the user via slider (population-water-scarcity-sensitivity).

\section{Sub-model 14: city-of-london-expansion}

This procedure spatially expands the City of London urban areas (landuse-category $=1$ ). Nirupama and Simonovic (2002) analyzed the process of urbanization around the City of London through the use of remotely sensed data over the period of three decades. The study divides land-use to 8 categories: woods, row crops and legumes, grasses, small 
grains or grains, fallow land, urban, homestead, and water. The study shows that London urbanized areas increased from $55.17 \mathrm{~km}^{2}$ in 1974 to $178.95 \mathrm{~km}^{2}$ in 2000 , Figure 30 . This gives the rate of $4.76 \mathrm{~km}^{2}$ or 528.97 patches per year and 44 patches per one time step. The model also calculates the population density in the City of London based on the current population and occupied city area, and depending on which particular socioeconomic scenario is analyzed, the user is allowed to modify accordingly the number of newly occupied cells in each time step.

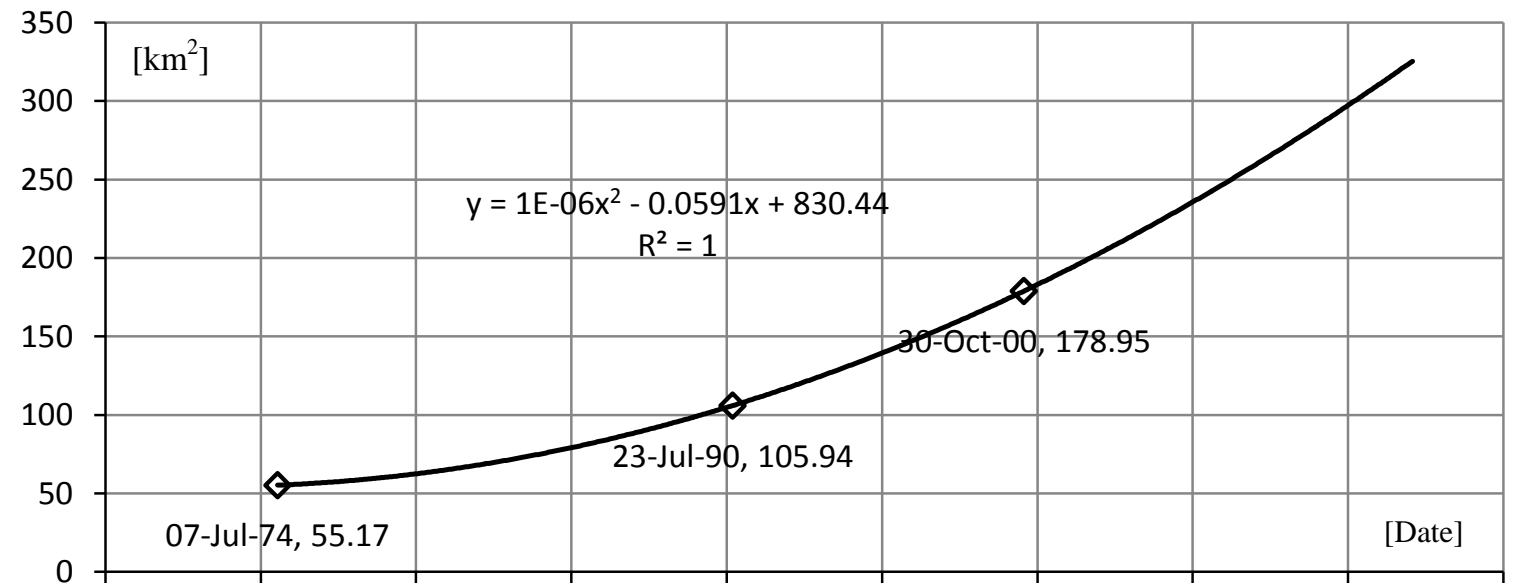

11-Jun-68 02-Dec-73 25-May-79 14-Nov-84 07-May-90 28-Oct-95 19-Apr-01 10-Oct-06 01-Apr-12 22-Sep-:

$\diamond$ London Urban Land Use —Poly. (London Urban Land Use )

Figure 30: Urbanization of the City of London

\section{Sub-model 15: ut-urbanization}

This sub-model defines the number of newly urbanized areas in the Upper Thames River basin (ut-urbanized-patches) in each time step. Since the City of London represents the most significant and largest urban center in this river basin, it is represented separately. Therefore, this procedure includes spatial expansion of all other seven urban centers, excluding London (landuse-category $=2,3,4,16,28,32,33,34,38$ and 39). Just like in the case of the City of London's spatial expansion, this procedure executes urbanization by converting the agricultural areas (patches), while other land-use categories are protected, such as forests, wetlands, recreational areas, etc. By changing the procedural 
structure, it is possible to modify the rules and allow urban expansion by converting other land-use categories, for example forest lands.

\section{Sub-model 16: reforestation}

One of the Upper Thames River Conservation Authority's (UTRCA's) long term objectives is to increase the percentage of forest lands within the basin to $30 \%$ of the total basin area. This sub-model represents the reforestation process in the UTRB, based on the inputs defined by the model user. The model user via slider (reforestation-rate) defines the number of patches dedicated to reforestation in each time step.

\section{Sub-model 17: subbasin-monthly-nonpermitted-demand}

The water withdrawals, not included in the PTTW database, must also be included in the water balance calculations. Each sub-basin contains a value of unpermitted water takings per day defined in a database (utsubbasins.shp). Depending on the type of use (unserviced domestic or agricultural), this procedure calculates the amount of water taken during the current time step (subbasin-monthly-nonpermitted-domestic-use and subbasinmonthly-nonpermitted-agriculture-use) from Table 24, by multiplying the annual value with an appropriate value from the seasonal variation list (nonpermitted-domesticseasonal-variation-list and nonpermitted-agriculture-seasonal-variation-list) in Table 25.

This sub-model includes one important assumption - quantities of unserviced domestic use include also amounts of water for dewatering purposes.

Table 24: Unpermitted and dewatering water use per sub-basin

\begin{tabular}{cccc}
\hline Sub-basin Name & $\begin{array}{c}\text { Private Use } \\
\text { and Dewatering } \\
{\left[\mathrm{m}^{3} / \mathrm{day}\right]}\end{array}$ & $\begin{array}{c}\text { Agricultural } \\
\text { Use } \\
{\left[\mathrm{m}^{3} / \mathrm{day}\right]}\end{array}$ & $\begin{array}{c}\text { Total } \\
{\left[\mathrm{m}^{3} / \mathrm{day}\right]}\end{array}$ \\
\hline Upper Avon River & 418 & 356 & 774 \\
Black Creek / Lower Avon River & 675 & 574 & 1249 \\
Cedar Creek & 120 & 195 & 315 \\
Flat Creek / Glengowan & 112 & 240 & 352 \\
Middle Thames River & 617 & 959 & 1576 \\
Wedway Creek / North Thames Above London & 977 & 961 & 1838 \\
Whirl Creek / North Mitchell & 685 & 907 & 1592 \\
Reynolds Creek & 364 & 377 & 741 \\
Thames River above Ingersoll & 442 & 456 & 898 \\
Thames River above Pittock Reservoir & 746 & 560 & 1606 \\
Trout Creek / North Thames River above St. Mary's & 500 & 376 & 1175 \\
Waubuno Creek / Dorchester & 1307 & 6831 & 1678 \\
\hline Total & 6963 & & 13794 \\
\hline
\end{tabular}


Table 25: Seasonal variation of unpermitted water use

\begin{tabular}{|c|c|c|c|c|c|c|c|c|c|c|c|}
\hline I & II & III & IV & V & VI & VII & VIII & IX & $\mathrm{X}$ & XI & XII \\
\hline \multicolumn{12}{|c|}{ nonpermitted-domestic-seasonal-variation-list } \\
\hline 0.0833 & 0.0833 & 0.0833 & 0.0833 & 0.0833 & 0.0833 & 0.0833 & 0.0833 & 0.0833 & 0.0833 & 0.0833 & 0.0833 \\
\hline \multicolumn{12}{|c|}{ nonpermitted-agriculture-seasonal-variation-list } \\
\hline 0 & 0 & 0 & 0.029 & 0.029 & 0.1229 & 0.4693 & 0.2979 & 0.321 & 0.321 & 0 & 0 \\
\hline
\end{tabular}

\section{Sub-model 18: subbasin-water-balance}

This sub-model firstly estimates the total water demand from all water users within the particular sub-basin (subbasin-total-water-demand) as a sum of agricultural, commercial, industrial, and municipal water supply demand (subbasin-agricultural-demand, subbasincommercial-demand, subbasin-industrial-demand, subbasin-watersupply-demand) and unpermitted water demand for domestic and agricultural use (subbasin-monthlynonpermitted-domestic-use and subbasin-monthly-nonpermitted-agriculture-use). Also, this model manages the long term water balance for each sub-basin based on the total water demand, groundwater recharge and streamflow.

subbasin-total-water-demand $=$ subbasin-agricultural-demand + subbasin-industrial-demand + subbasin-commercial-demand + subbasin-watersupply-demand + subbasin-monthly-nonpermitteddomestic-use + subbasin-monthly-nonpermitted-agriculture-use

subbasin-current-water-balance $=$ subbasin-current-water-balance + subbasin-groundwaterrecharge - subbasin-total-water-demand

\section{Sub-model 19: municipal-water-balance}

This sub-model calculates the total water demand (municipal-total-water-demand) on the county level by summarizing all sub-basins in the county.

municipal-curent-water-balance $=$ sum [ subbasin-current-water-balance $]$ of subbasin-tomunicipal-link-neighbors

\subsubsection{Agent-based model parameterization and calibration}

The simulation models generate system behavior as a consequence of certain input conditions. The main idea behind any modelling study is to reproduce observed patterns 
of system behavior over the time and space. Depending on the main model objectives, models typically do not represent all processes that cause the real system to change over time. This simplified representation means that only a limited number of variables are used to represent the real system. Having that in mind, two stages of model development process are particularly important: model parameterization and model calibration.

Model parameters represent the specific values in the mathematical equations and algorithms that are used to describe model structure. The process of determining model parameters is called the model parameterization (Railsback and Grimm, 2011). Once the model is parameterized, a modeler continues with the model calibration process. Calibration is a process of finding the optimal values for identified calibration parameters and it is done by analyzing what parameter values drive the model to reproduce patterns of observed system behavior.

In agent-based modeling, the calibration process is important for three reasons (Railsback and Grimm, 2011). First, this process adjusts the model to match empirical observations as closely as possible. Second, this process improves the estimation of parameters that cannot be directly defined and evaluated. And finally, calibration process also tests the structural integrity of an often complex agent-based model.

Agent-based models typically represent very complex systems which contain information about the system stored in system entities, state and global variables, and, most importantly - sub-models. Since an agent-based model represents a finite collection of subsequent sub-models, all sub-models are parameterized and calibrated separately. As a result, due to inherited complexity, agent-based models compared to traditional modeling methods contain more equations and processes, but the calibration process includes only a fine modification of a small number of sensitive parameters. Consequently, the most important strategy to make the agent-based model reliable and credible is to develop and test each existing sub-model independently (Railsback and Grimm, 2011).

Developed spatially explicit socio-economic agent-based model for the Upper Thames River basin employs two solutions to sub-model parameterization. First, it uses appropriate parameter values from the external and official databases. Also, all 
parameters of socio-economic sub-models represent the real quantities and values that can be measured empirically. Second, particular sub-models (such as population dynamics sub-model for example) use parameters of existing models of the same process that have been described and analyzed. 


\section{Chapter 5}

\section{Results and discussion}

This section has two main objectives. First, it introduces scenarios used to test the system response to different combinations of climate and socio-economic conditions. Second, it provides the discussion of the results obtained by simulating the Integrated HydrologicSocio-Economic Model developed for the Upper Thames River basin (IHSEM-UTRB) case study.

\subsection{Simulation scenarios}

The main idea behind the simulation models is to help us estimate the resulting system behavior based on a set of assumptions and initial system inputs. In water resources management, simulation models are designed to test the outcomes of different policies and strategies through a set of scenarios. A simulation scenario contains a predefined set of rules, parameters and strategies embedded in the model. Even though the designed multi-method model allows a game-like interactive control of all parameters throughout the simulation, thus allowing for timely adaptation of processes that might lead to system instability, a two sets of scenarios are predefined to investigate the two distinct conditions in the Upper Thames River basin. Each run of Integrated Hydrologic-Socio-Economic Model for the Upper Thames River basin (IHSEM-UTRB) simulates one combination of two groups of scenarios: climate and socio-economic scenarios.

\section{Development of climate scenarios}

Climate scenarios are considered to be the main driver of the hydrologic component and the system itself. In current engineering practices, the design of municipal water management infrastructure, such as dykes, stormwater systems, culverts, or drains, fundamentally depend on climate conditions. Therefore, information is needed to describe how extreme precipitation events are expected to change as climate changes. Previous analysis of the effects of changing climate conditions for the Upper Thames River basin shows that the frequency of flooding will be altered depending on the 
precipitation magnitude considered, Prodanovic (2007). Figures 31 and 32 illustrate the statistical analysis for three climate scenarios used to describe historic, wet, and dry conditions and show the similar values of peak flow for both Gumbel and Pearson III distributions up to the 100 year return period.
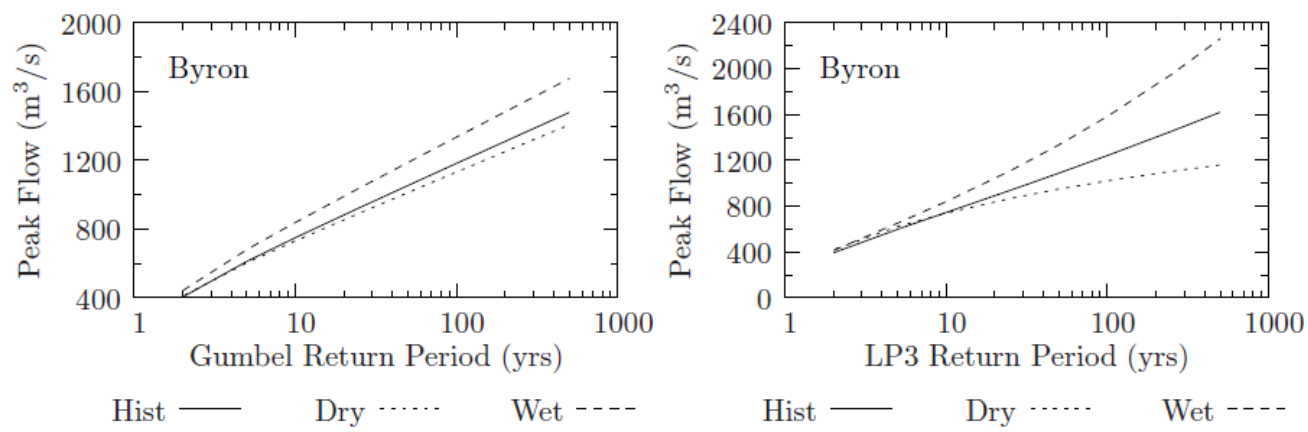

Figure 31: Flood frequency analysis under different climate conditions, Byron station
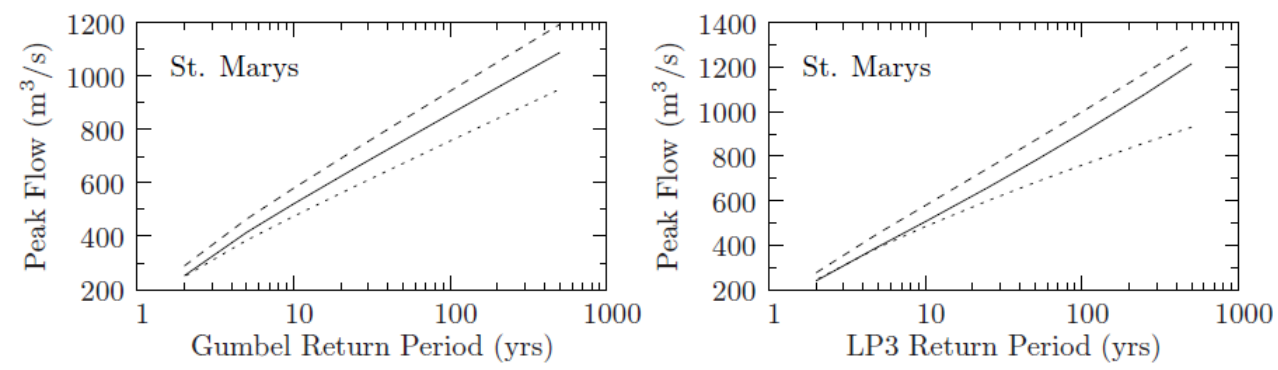

Hist $-\quad$ Dry $\cdots \cdot$ Wet ----

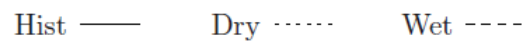

Figure 32: Flood frequency analysis under different climate conditions, St.Marys station

Depending on the analyzed climate scenario, the same figures reveals significant variations of peak flow values for the same return periods but different climate scenarios. For example, the flow of $900 \mathrm{~m}^{3} / \mathrm{s}$ at Byron station, under the analyzed historic scenario, has a return period of 33 years. But in the wet climate scenario, the same flow has a significantly lower return period of only 17 years. In contrast, the return period of the same flow in case of dry climate scenario is 65 years. These three values represent a significant range that brings additional uncertainty to engineering practices. On the other side, the same study shows that low flow conditions will be almost identical as those 
currently observed, Figures 33 and 34 . These figures show the annual minimum seven day and monthly flow for two stations (St. Marys and Byron) for three climate scenarios.

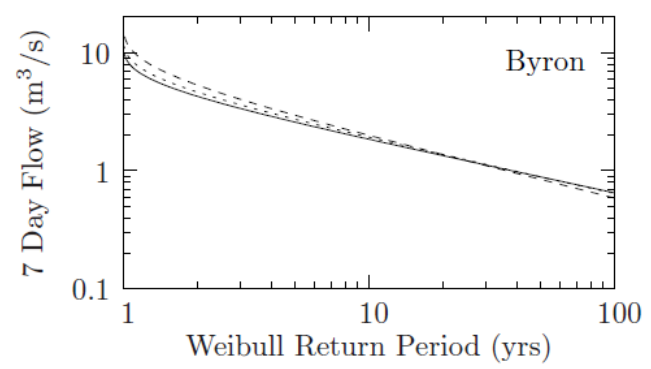

Hist - Dry $\cdots .$. Wet --- -

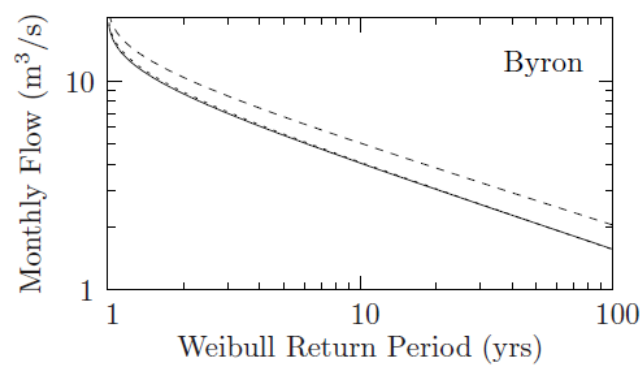

Hist $-\quad$ Dry $\cdots . . . \quad$ Wet $-\cdot-$ -

Figure 33: Low flow frequency analysis under different climate conditions, Byron station

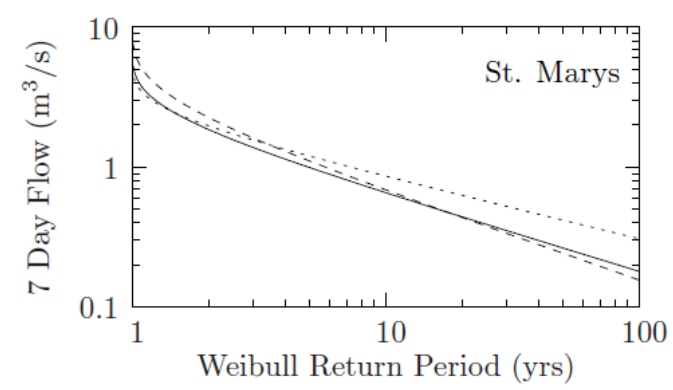

Hist - Dry $\cdots .$. Wet $-\cdot--$

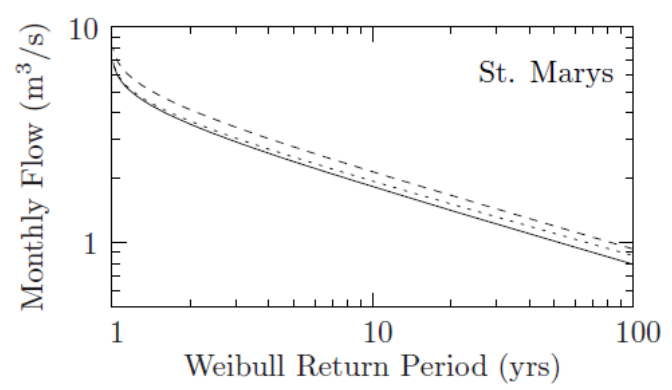

Hist $-\quad$ Dry $\cdots \cdot$... Wet ---

Figure 34: Low flow frequency analysis under different climate conditions, St. Marys station

In order to analyze the effects of climate change on local water resources and the hydrologic response to changing climate conditions, Prodanovic (2007) uses the inverse approach that applies the weather generator algorithm to produce arbitrary long-time series of climatic input data (temperature and precipitation). The weather generator algorithm utilizes different shuffling and perturbation mechanisms with a local-climate time series to create a statistically similar and locally applicable set of climate conditions for the future. Generally speaking, there are two types of weather generators: parametric and non-parametric. Parametric generators are stochastic tools that produce weather data by assuming a probability distribution function and a number of site specific parameters for the variable of interest. In contrast, non-parametric do not include site specific 
parameters or distribution assumptions and are based purely on shuffling and sampling algorithms.

Prodanovic(2007) uses the outputs from global circulation model (GCM) simulations to condition the input data used by the weather generator and thus create the climate change scenarios. Change fields for each climate scenario are computed based on the percent difference from the baseline case for monthly precipitation, minimum and maximum temperature. To calculate the change fields, monthly values of global data are averaged for all years of output. Once the change fields are calculated, the climate scenarios are defined by multiplying or adding the locally observed climate data for a number of stations and the monthly percentage change values.

Based on the IPCC (2001) two scenario story lines B1 and B2 are used. The two scenarios extract the necessary information provided by outputs of CSIROM $2 \mathrm{~kb}$ and CCSRNIES global circulation models. The B1 story line describes a global change towards service and information based economies and promotes the use of clean technologies. In contrast, the storyline B2 stresses local initiatives to socio-economic and environmental security and predicts technological development towards regional social equity and environmental protection. The projections made by above mentioned GCMs are used as inputs into the weather generator to obtain multiple realizations of future climate. While scenario CCSERNIES B21 (wet) is used to describe the upper range of possible magnitude of precipitation, CSIROM $2 \mathrm{~kb} \mathrm{B11(dry)}$ is used to represent the lower range. The obtained change fields are presented in Table 26. Climate change scenarios developed using this method enables analysis of both, local and global climatic data in order to predict the possible future conditions. For the purpose of his study, Prodanovic (2007) uses the weather generator to simulate daily data for continuous hydrologic modeling and hourly data for event modeling. The continuous model is used to analyze the effects of climate change on the frequencies of low flows and droughts, while the event model analyses the flood frequencies. The K-Nearest Neighbor weather generator developed by Sharif and Burn $(2004,2006,2007)$ runs on a daily time step and is not adequate for creating short-duration high-intensity storm events that cause flooding. 
Table 26: Monthly precipitation and temperature change fields

\begin{tabular}{ccccccc}
\hline & \multicolumn{3}{c}{ CSIROM2kb B11 (Dry) } & \multicolumn{3}{c}{ CCSRNIES B21 (Wet) } \\
\hline Month* & $\mathrm{P}(\%)$ & $\mathrm{T}\left({ }^{\circ} \mathrm{C}\right)$ & $\mathrm{T}_{+}\left({ }^{\circ} \mathrm{C}\right)$ & $\mathrm{P}(\%)$ & $\mathrm{T}\left({ }^{\circ} \mathrm{C}\right)$ & $\left.\mathrm{T}_{+}+{ }^{\circ} \mathrm{C}\right)$ \\
\hline Jan & 10.41 & 4.43 & 3.35 & 17.67 & 6.84 & 6.84 \\
Feb & 5.74 & 3.29 & 3.18 & 6.38 & 4.95 & 5.24 \\
Mar & -0.98 & 4.52 & 7.02 & 15.07 & 5.83 & 6.43 \\
Apr & -11.41 & 5.78 & 4.77 & 22.84 & 5.91 & 6.51 \\
May & 19.19 & 4.50 & 1.88 & 24.14 & 5.60 & 6.09 \\
Jun & 4.56 & 3.32 & 2.43 & 18.55 & 4.25 & 4.60 \\
Jul & 5.87 & 3.59 & 4.04 & 5.03 & 4.25 & 4.14 \\
Aug & 15.32 & 4.09 & 2.41 & 7.88 & 4.85 & 5.02 \\
Sep & -6.65 & 2.11 & 3.66 & 4.27 & 4.57 & 5.20 \\
Oct & 5.39 & 3.11 & 2.51 & -11.51 & 4.47 & 5.51 \\
Nov & -6.12 & 4.64 & 3.34 & -15.55 & 5.26 & 6.13 \\
Dec & 5.09 & 1.43 & 3.06 & -3.10 & 5.80 & 6.43 \\
\hline Aver
\end{tabular}

* Average percent difference from base case for period $2040-2069$ using grid cell centered at (43.01, 78.75); P Precipitation; T- Minimum temperature; T+ Maximum temperature

Modified version created by Wey (2006) takes synthetic daily data coupled with historically observed hourly rainfall and disaggregates it to generate hourly rainfall data. The initial version of the weather generator produces 100 years of daily data for three climate scenarios, for each climate station in the Upper Thames River basin, Table 27. They use historically observed precipitation and temperature data for the period between 1964 and 2001 to condition the weather generator. Therefore, each station contains 100 years of 365 days per year data, giving in total 36,500 days of precipitation for each of the three climate scenarios (historic, dry, wet). To disaggregate precipitation data into hourly, only days with more than $25 \mathrm{~mm}$ of precipitation are taken into consideration. A single critical event from each year is selected and input to the event model. Peak flows from the 100 events are used in flood flow analysis to estimate the potential impact of changing climatic conditions on the hydrologic flow regimes. On the other hand, the continuous hydrologic model uses the synthetic data generated for the same fifteen stations for the same three scenarios. 
Table 27: Generated meteorological data

\begin{tabular}{clccclcc}
\hline No. & Station & Latitude & Longitude & No. & Station & Latitude & Longitude \\
\hline 1 & Blythe & $43^{\circ} 43^{\prime}$ & $-81^{\circ} 22^{\prime}$ & 9 & London & $43^{\circ} 01^{\prime}$ & $-81^{\circ} 09^{\prime}$ \\
2 & Dorchester & $43^{\circ} 00^{\prime}$ & $-81^{\circ} 01^{\prime}$ & 10 & St. Thomas & $43^{\circ} 46^{\prime}$ & $-81^{\circ} 12^{\prime}$ \\
3 & Embro & $43^{\circ} 15^{\prime}$ & $-80^{\circ} 55^{\prime}$, & 11 & Stratford & $43^{\circ} 22^{\prime}$ & $-81^{\circ} 00^{\prime}$ \\
4 & Exeter & $43^{\circ} 15^{\prime}$ & $-81^{\circ} 30^{\prime}$ & 12 & Tavistock & $43^{\circ} 19^{\prime}$ & $-80^{\circ} 49^{\prime}$ \\
5 & Foldens & $43^{\circ} 01^{\prime}$ & $-80^{\circ} 46^{\prime}$ & 13 & Waterloo & $43^{\circ} 28^{\prime}$ & $-80^{\circ} 31^{\prime}$ \\
6 & Fullarton & $43^{\circ} 23^{\prime}$ & $-81^{\circ} 12^{\prime}$ & 14 & Woodstock & $43^{\circ} 08^{\prime}$ & $-80^{\circ} 46^{\prime}$ \\
7 & Glen Allan & $43^{\circ} 40^{\prime}$ & $-80^{\circ} 43^{\prime}$ & 15 & Wroxeter & $43^{\circ} 52^{\prime}$ & $-80^{\circ} 09^{\prime}$ \\
8 & Ilderton & $43^{\circ} 03^{\prime}$ & $-81^{\circ} 25^{\prime}$ & & & & \\
\hline
\end{tabular}

The application of this methodology has produced three sets of climate scenarios used for the analysis: Historical, Wet and Dry scenarios.

Historical Climate Scenario. In this case, the historical records have not been altered, only extended to the period between January 1st, 2000 and January 1st, 2020, based on regional hydro-climatic data from the 1964-2001 period.

Wet Climate Scenario. This scenario represents the wetter future climate that is warmer than normal. It is obtained by selecting the weather generator realization that projects highest mean rainfall. This scenario modifies the historical data by applying change fields resulting from CCSRNIES B21, as shown in Table 26.

Dry Climate Scenario. It represents the drier and cooler climate future scenario. It is obtained by selecting the weather generator realization that projects the lowest mean rainfall in future. This scenario alters the historic record with change fields given in Table 26.

In order to assess the system response to presented range of climate variability, this research adopts the identical set of climate scenarios. However, it should be noted that Prodanovic (2007) simulates precipitation, daily and hourly, for all stations from January 01, 2001 to December 31, 2108, and makes an output of 108 years. It is estimated that such a long period is required for assessing the events, floods and droughts, with return period over 100 years. On the other side, this research limits the simulation time to 20 years in the period between January 1st, 2000 and January 1st, 2020. This is done for two main reasons. First, the latest available Permits to Take Water (PTTW) database is 
updated in 2014, and, therefore, the longer simulation times would require a number of arbitrary assumptions regarding the water use in the Upper Thames River basin. On the other side, this research relies on available land-use maps available for the same period of time.

\section{Development of socio-economic scenarios}

On the other hand, the socio-economic scenarios are used to describe different policy options in the system. Generally speaking, socio-economic process can be divided into two general categories: processes with spatial implications that directly affect the environment (such as land-use change as a result of urbanization, deforestation and reforestation) and processes related to water consumption (population dynamics, economic sub-models, etc.). Two socio-economic scenarios are developed for the purpose of this research: the baseline and infinite natural resources socio-economic scenarios.

The baseline socio-economic scenario studies the system behavior under regular socioeconomic conditions used to design all sub-models. These conditions are derived from the observed historical analysis, and this scenario assumes that identical socio-economic trends will be occurring in the near future during the period of simulation (January 1, 2000 - January 1, 2020). The simulation time is 240 time steps representing 20 years, where each time step is equal to one month. It should be noted that this is done for two main reasons. First, the latest available database of Permits to Take Water is updated in 2014, and, therefore, the longer simulations would require a number of arbitrary assumptions to describe the future water demand in the UTRB. On the other side, this research uses available land-use maps for the same period of time. Despite the fact that the model is designed in a way that allows the user to modify the parameters during the course of simulation, in this combination of scenarios, all socio-economic parameters remain constant throughout the simulation. Table 28 shows the concrete model parameters selected via existing switches, sliders and choosers used to describe the baseline socio-economic scenario. Detailed explanation of each model switch, slider and chooser, as well as the sub-models that are used to describe socio-economic activities 
within the basin, are given in the Chapter 3 . The baseline scenario assumes that all infrastructural elements, in this case two primary water supply systems, operate by full capacity, and includes the relatively high per capita water consumption observed in Canada. Also, the rates of urbanization remain identical during the course of simulation.

Table 28: Model parameters for the baseline socio-economic scenario

\begin{tabular}{|c|c|}
\hline Parameter & Value \\
\hline subbasin-water-balance-1 & 20 \\
\hline ut-birth-rate & 0.009 \\
\hline show-links & FALSE \\
\hline london-spatial-expansion & 68 \\
\hline subbasin-water-balance-2 & 14 \\
\hline elgin-area-coefficient & 1 \\
\hline show-city-agents & FALSE \\
\hline commercial-consumption-coefficient & 1 \\
\hline industrial-water-price & 1.75 \\
\hline show-subbasin-labels & TRUE \\
\hline hydrology-scenario & "Dry/Wet/Historic" \\
\hline industrial-consumption-coefficient & 1 \\
\hline mixed-yield-tonnes-per-hectare & 15 \\
\hline show-agriculture & FALSE \\
\hline corn-yield-tonnes-per-hectare & 10.1 \\
\hline nonpermitted-agriculture-use-multiplier & 1 \\
\hline subbasin-water-balance-3 & 27 \\
\hline agricultural-consumption-coefficient & 1 \\
\hline mixed-dollars-per-tonnes & 50 \\
\hline average-per-capita-per-day-water-consumption & 420 \\
\hline ut-shift & 0.325 \\
\hline ut-min-birth-rate & 0.02 \\
\hline show-municipalities & FALSE \\
\hline barley-dollars-per-tonnes & 218 \\
\hline barley-yield-tonnes-per-hectare & 3.2 \\
\hline show-subbasins & FALSE \\
\hline ut-max-death-rate & 0.025 \\
\hline ut-urbanized-patches & 31 \\
\hline huron-lake-coefficient & 1 \\
\hline reforestation-rate & 100 \\
\hline show-dem & FALSE \\
\hline corn-dollars-per-tonnes & 232 \\
\hline agricultural-commodity-price-variation & 1 \\
\hline hay-dollars-per-tonnes & 193 \\
\hline nonpermitted-domestic-use-multiplier & 1 \\
\hline population-water-scarcity-sensitivity & 0.05 \\
\hline hay-yield-tonnes-per-hectare & 5.5 \\
\hline show-gauging-stations & FALSE \\
\hline ut-min-death-rate & $5.00 \mathrm{E}-04$ \\
\hline ut-max-birth-rate & 0.025 \\
\hline
\end{tabular}

On the other side, one scenario is created to represent extreme socio-economic conditions in the basin. The infinite natural resources scenario analyzes the effects of careless use of 
always-abundant natural resources. Since the Upper Thames River basin is already effectively managed by the different levels of authorities, this scenario is not likely to take place, but it helps us analyze the implications of the belief that natural resources, water and land, are limitless and indestructible. In this infinite natural resources scenario (ii), the focus is primarily placed on economic growth and a high living standard, while disregarding the state of natural resources. As in the previous case, combinations of one socio-economic and three climate scenarios analyze how different climate conditions can potentially affect the local socio-economic environment. Also, this combination simulates how extreme socio-economic trends may affect the existing hydrologic regime. Table 29 shows the concrete model parameters defined via existing switches, sliders and choosers used to describe the infinite natural resources socio-economic scenario. A detailed explanation of each model switch, slider and chooser, as well as the sub-models used to describe socio-economic activity within the basin, is given in Chapter 4. In contrast to the base line scenario, this scenario assumes that two primary water-supply systems operate in extended capacity in order to meet the increasing water demand, Table 29. It also includes the relatively high per-capita water consumption observed in Canada. 
Table 29: Specific model parameters used to describe infinite natural resources socioeconomic scenario

\begin{tabular}{cc}
\hline Parameter & Value \\
\hline mixed-yield-tonnes-per-hectare & 15 \\
show-agriculture & FALSE \\
ut-birth-rate & 0.009 \\
nonpermitted-domestic-use-multiplier & 1.3 \\
nonpermitted-agriculture-use-multiplier & 1.4 \\
ut-min-death-rate & $5.00 \mathrm{E}-04$ \\
show-links & FALSE \\
subbasin-water-balance-1 & 20 \\
show-dem & FALSE \\
barley-dollars-per-tonnes & 218 \\
subbasin-water-balance-2 & 14 \\
huron-lake-coefficient & 1.3 \\
hay-dollars-per-tonnes & 193 \\
industrial-water-price & 1 \\
pttw-permit-extension & 2 \\
mixed-dollars-per-tonnes & 50 \\
population-water-scarcity-sensitivity & 0.15 \\
average-per-capita-per-day-water-consumption & 420 \\
corn-yield-tonnes-per-hectare & 10.1 \\
industrial-consumption-coefficient & 1.35 \\
corn-dollars-per-tonnes & 232 \\
agricultural-commodity-price-variation & 1 \\
hay-yield-tonnes-per-hectare & 5.5 \\
show-city-agents & FALSE \\
london-spatial-expansion & 120 \\
agricultural-consumption-coefficient & 1.45 \\
ut-min-birth-rate & 0.02 \\
subbasin-water-balance-3 & 27 \\
show-gauging-stations & FALSE \\
elgin-area-coefficient & 1.35 \\
show-subbasins & FALSE \\
reforestation-rate & 20 \\
ut-max-death-rate & 0.025 \\
show-subbasin-labels & TRUE \\
ut-urbanized-patches & 110 \\
show-municipalities & FALSE \\
ut-shift & 3.25 \\
hydrology-scenario & 0.325 \\
barley-yield-tonntion-coefficient & 0.025 \\
commercial-consumpectare & \\
\hline & \\
utiot/Dry" \\
\hline
\end{tabular}

Different combinations of three climate and two socio-economic scenarios presented in Table 30 give a total of six experiments used to simulate the system response to a wide range of different conditions. Each combination helps us see how different climate conditions can potentially affect the local socio-economic environment. At the same time, 
we are able to simulate how projected and expected socio-economic trends may affect existing hydrologic regime.

Table 30: Six combinations of climate and socio-economic scenarios

\begin{tabular}{|c|c|c|c|}
\hline & & \multicolumn{2}{|c|}{ Set of socio-economic scenarios } \\
\hline & & Baseline scenario (i) & $\begin{array}{c}\text { Infinite natural resources } \\
\text { scenario (ii) }\end{array}$ \\
\hline Set of & Historic climate (a) & Experiment 1 & Experiment 4 \\
\hline hydrologic & Wet climate (b) & Experiment 2 & Experiment 5 \\
\hline scenarios & Dry climate (c) & Experiment 3 & Experiment 6 \\
\hline
\end{tabular}

Following sections describe the obtained results from six experiments.

\subsection{Experiment 1: the baseline socio-economic scenario (i) and historic climate conditions (a)}

The integrated hydrologic and socio-economic model defines water as the main factor of the regional social and economic development. However, due to projected high-usage rates, increased demand, and declining supplies in some areas of the basin caused by changing climate conditions, water may become the limiting factor to future growth and expansion. In this section, the results obtained for the combination of the baseline socioeconomic (i) and historic climate conditions (a) in the UTRB are shown.

Figures 35 - 39 present the spatial manifestation of urbanization and land use change (deforestation and reforestation) processes in five-year time steps $(1,61,121,181$, and 241). In these figures, grey patches represent the urbanized areas as on January $1^{\text {st }}, 2000$, while white patches (cells) represent newly urbanized areas in the basin during the course of simulation. Green patches represent forested lands, and these maps show further reforestation promoted by local conservation authorities. Furthermore, these figures show all active water users (industrial, agricultural, commercial and municipal water supply) in the respective time steps. Figure 40 presents the actual rates of land-use change in $\mathrm{km}^{2}$, showing the steady decline of agricultural lands and increase of residential and forest land use. Projected economic development in the region is expected to create more jobs and attract more people to the region, and, therefore, Figure 41 illustrates the steady dynamics of London's population growth in the twenty-year period. On the other hand, Figure 42 shows the rate of population density change in the City of London. As 
demographic growth intensifies, so do the rates of urbanization and development. As a result, areas around London are under development, and agricultural land is being converted to residential and economic uses. Also, on the level of the whole basin, areas closest to the existing urban centers and other infrastructural features (such as roads, highways, etc.) are experiencing similar development, Figures 35 - 39.

Figure 43 shows all categories of active water users in each time step according the PTTWs database. The rates of water consumption and their periods of validity are extracted from the PTTW database and remain unmodified during the course of simulation. As can be expected, the number of effective licenses slowly declines after 2014, at the time step 168, as the result of unchanged license expiration dates. Rates of non-permitted domestic and agricultural use are also imported from the database, and their consumption multiplier coefficients are equal to one. Based on the individual demand, Figure 44 presents the seasonal variation of water demand for active industrial, agricultural and commercial water users. The next two figures, Figures 45 and 46 show economic activities per sector (industrial and agricultural) in terms of Canadian dollars based on the quantities of used water, as per already-described economic sub-models. Economic decline in industrial sector observed in Figure 45 is a result of decreased water demand recorder in the permit to take water database. Figure 47 shows economic revenue in dollars of the currently cultivated areas in the basin, taking account the current crop patterns, yields, and crop prices. Since the urbanization and reforestation processes are occurring on account of agricultural land, this figure shows a steady decline in economic revenues.

Figure 48(a) shows the obtained River Thames median monthly flow rates at Byron station for historic climate conditions. The median flow rate for the period of simulation is $25.46 \mathrm{~m}^{3} / \mathrm{s}$, while the average flow is $30.02 \mathrm{~m}^{3} / \mathrm{s}$. Figure 49 (a) presents the median monthly flow rates at Ingersoll. The median flow rate for the period of simulation is $9.33 \mathrm{~m}^{3} / \mathrm{s}$, while the average flow is $11.17 \mathrm{~m}^{3} / \mathrm{s}$. Finally, Figure 50 (a) shows the same results at St.Marys where the median flow rate is $3.30 \mathrm{~m}^{3} / \mathrm{s}$, and the average is $4.01 \mathrm{~m}^{3} / \mathrm{s}$. The detailed comparison between different climate scenarios (historic, wet and dry) for all three stations is given in Chapter 5.8. In addition to surface flows, one of the most 
important impacts of land-use change and urbanization on hydrologic regime is alteration of groundwater recharge rates. As a result of urbanization, less water is infiltrated to the groundwater aquifers that many municipalities use for their drinking water supply. At the same time, industry and agriculture use the groundwater for manufacturing and food production. It is expected that quantities of available groundwater will decline over time and slow down future economic and social growth. This model represents the groundwater recharge as the volume of precipitation that infiltrates the ground water aquifers in any given time step. Figures 51(a), 52(a) and 53(a) show the seasonal variations of ground water recharge rates and total water demand for three counties (Middlesex, Oxford and Perth) in the Upper Thames River basin for historic climate scenario. It must be noted that since this model does not include a component that calculates the impacts of groundwater and surface water withdrawals typically required to assess the state of local aquifers, ground water recharge rates are compared to the water demand. It is preferred that the water demand remains smaller than the recharge rates, to avoid additional water withdrawals and extraction of reserves from groundwater aquifers. At this spatial scale, results do not show any significant disproportion between natural recharge and demand rates for this climate scenario. This suggests that groundwater aquifers in these three counties are not in danger of overexploitation under the projected socio-economic and climate conditions. However, the situation drastically changes at the lower spatial scales (the sub-basin level). Based on the state of local socio-economic activity in each subbasin, three sub-basins are chosen for detailed analysis - Middle Thames (sub-basin ID 14 selected via chooser), North Mitchell (sub-basin ID 20 selected via chooser), and River Bend (sub-basin ID 27 selected via chooser).

According to the PTTW database and accounted non-serviced water demand, Middle Thames and North Mitchell sub-basins contain a small number of water permits as a result of relatively insignificant local socio-economic activity. Expectedly, both subbasins have enough resources to manage their groundwater aquifers sustainably. For both basins, Figure 54(a) and Figure 56(a) compare the monthly rates of groundwater recharge to monthly total water demand for described socio-economic conditions for historic climate scenario. Cumulative water balance accounted for both sub-basins, presented in Figures 55(a) and 57(a), does not show any threat of overutilization. The observed trends 
suggest that, in the case of existing climate conditions, the two sub-basins should be able to support future economic growth, at least in terms of renewable water resources.

On the other hand, the situation is considerably different in the Southwestern parts of the Upper Thames River basin. As a result of strong local socio-economic activity, mainly intensive agricultural practices, River Bend sub-basin has issued a significant number of water permits. Figure 58(a) shows monthly variation in groundwater demand and groundwater recharge rates, and reveals significant pressure on local water resources even in current climate conditions. Long-term cumulative water balance, presented in Figure 59(a), reveals that the local groundwater recharge rates are not sufficient to sustainably replenish groundwater aquifers due to extensive water withdrawals. This means that, in the future, with current climate conditions, local aquifers will be exposed to a severe pressure. However, this conclusion should be further analyzed, as the model does not account for interactions between local aquifers and large water bodies, such as the neighboring Great Lakes.

\subsection{Experiment 2: the baseline socio-economic scenario (i) and wet climate conditions (b)}

This scenario examines the system behavior under the baseline socio-economic and wet climate conditions. The wet climate scenario uses historical data modified to represent a climate that is wetter and warmer than normal, while the amount of precipitation is increased. Since the model parameters used to describe the baseline socio-economic scenario remain unchanged, as presented in Table 29, the socio-economic results are identical to the Experiment 1 and presented in Figures 35 to 47. However, the main difference refers to changes in hydrologic regime. Figure 48(b) shows the obtained River Thames median monthly flow rates at Byron station for wet climate conditions. The median flow rate for the period of simulation is $27.84 \mathrm{~m}^{3} / \mathrm{s}$, while the average flow is $33.25 \mathrm{~m}^{3} / \mathrm{s}$. Figure 49 (b) presents the median monthly flow rates at Ingersoll. The median flow rate for the period of simulation is $9.88 \mathrm{~m}^{3} / \mathrm{s}$, while the average flow is $12.05 \mathrm{~m}^{3} / \mathrm{s}$. Finally, Figure 50 (b) shows the same results at St.Marys where the median flow rate is $3.49 \mathrm{~m}^{3} / \mathrm{s}$, and the average is $4.58 \mathrm{~m}^{3} / \mathrm{s}$. Figures $51(\mathrm{~b}), 52(\mathrm{~b})$ and $53(\mathrm{~b})$ show the seasonal variations of ground water recharge rates and total water demand for three counties 
(Middlesex, Oxford and Perth) in the Upper Thames River basin for the wet climate scenario. Just like in the case of the historic climate conditions, results at this spatial scale do not show any significant imbalance between natural recharge and water demand rates. This suggests that groundwater aquifers in these three counties are not in danger of overexploitation under the projected socio-economic and wet climate conditions. Similarly to the historic climate condition, the situation is slightly different at the lower spatial scales (the sub-basin level). Figure 54(b) and Figure 56(b) compare the monthly rates of groundwater recharge to monthly total water demand for described socioeconomic conditions for wet climate scenario for two sub-basins, Middle Thames and North Mitchell. Figures 55(b) and 57(b) present the cumulative water balance accounted for both sub-basins (Middle Thames and North Mitchell respectively) and do not demonstrate any signs of overutilization. The observed trends suggest that, in the case of wetter climate conditions, the two sub-basins are able to support future economic growth, at least in terms of renewable water resources.

On the other hand, compared to the historic climate scenario, the situation at River Bend is slightly better. Figure 58(b) shows monthly variation in groundwater demand and groundwater recharge rates, and illustrates still present pressure on local water resources, even in the wet climate conditions. Long-term cumulative water balance, presented in Figure 59(b), demonstrates that the local groundwater recharge rates are still not sufficient to sustainably replenish groundwater aquifers due to extensive water withdrawals.

\subsection{Experiment 3: the baseline socio-economic scenario (i) and dry climate conditions (c)}

This scenario couples the baseline socio-economic and dry climate conditions. The dry climate scenario describes a drier and cooler climate, which might lead to dry, spells and droughts. The model parameters, shown in Table 28, used to define the baseline socioeconomic scenario remain unmodified, and the socio-economic results are presented in Figures 35 to 47 . On the other side, the hydrologic regime experiences some change. Figure 48(c) shows the obtained River Thames median monthly flow rates at Byron station for dry climate conditions. The median flow rate for the period of 20 years is 
$25.62 \mathrm{~m}^{3} / \mathrm{s}$, while the average flow is $27.65 \mathrm{~m}^{3} / \mathrm{s}$. Figure $49(\mathrm{c})$ presents the median monthly flow rates at Ingersoll. The median flow rate for the period of simulation is $8.35 \mathrm{~m}^{3} / \mathrm{s}$, while the average flow is $10.75 \mathrm{~m}^{3} / \mathrm{s}$. Finally, Figure 50 (c) shows the same results at St.Marys where the median flow rate is $3.02 \mathrm{~m}^{3} / \mathrm{s}$, and the average is $3.61 \mathrm{~m}^{3} / \mathrm{s}$. Figures 51(c), 52(c) and 53(c) show the seasonal variations of ground water recharge rates and total water demand for three counties, Middlesex, Oxford and Perth, in the basin for dry climate scenario. The results at county spatial scale illustrate only occasional imbalance between natural recharge and demand rates. This suggests that in long-terms groundwater aquifers in these three counties are not in danger of overexploitation even in dry climate conditions. Similarly to the historic climate condition, the situation is significantly different at the sub-basin level spatial scales. Figure 54(c) and Figure 56(c) compare the monthly rates of groundwater recharge to monthly total water demand for described socio-economic conditions for dry climate scenario for two sub-basins, Middle Thames and North Mitchell. These figures show only the seasonally higher water demand than the groundwater recharge. Figures 55(c) and 57(c) present the cumulative water balance accounted for both sub-basins (Middle Thames and North Mitchell respectively) and, in long terms, do not show signs of overexploitation. The observed trends suggest that, in the case of drier climate conditions, the two sub-basins should be able to support future economic growth. On the other hand, compared to the previous climate scenarios, the situation at River Bend sub-basin is alarming. Figure 58(c) shows monthly variation in groundwater demand and groundwater recharge rates, and reveals more significant seasonal pressures on the local water resources. Long-term cumulative water balance, presented in Figure 59(c), demonstrates that the local groundwater recharge rates, if inappropriately managed, can be inadequate to sustainably replenish groundwater aquifers. 


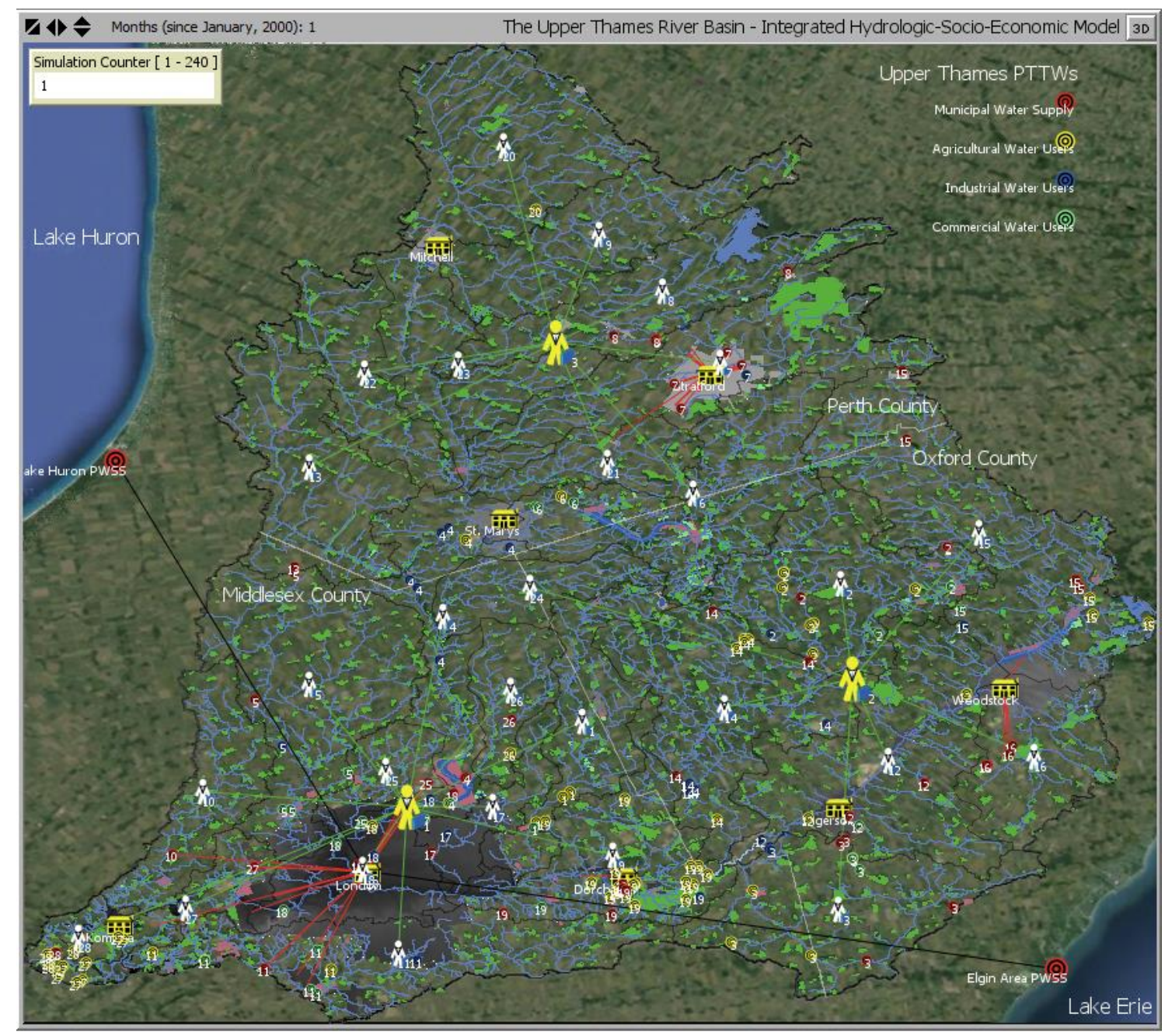

Figure 35: Dynamics of land use change and active water users in the Upper Thames River basin at time step 1 (January, 2000) 


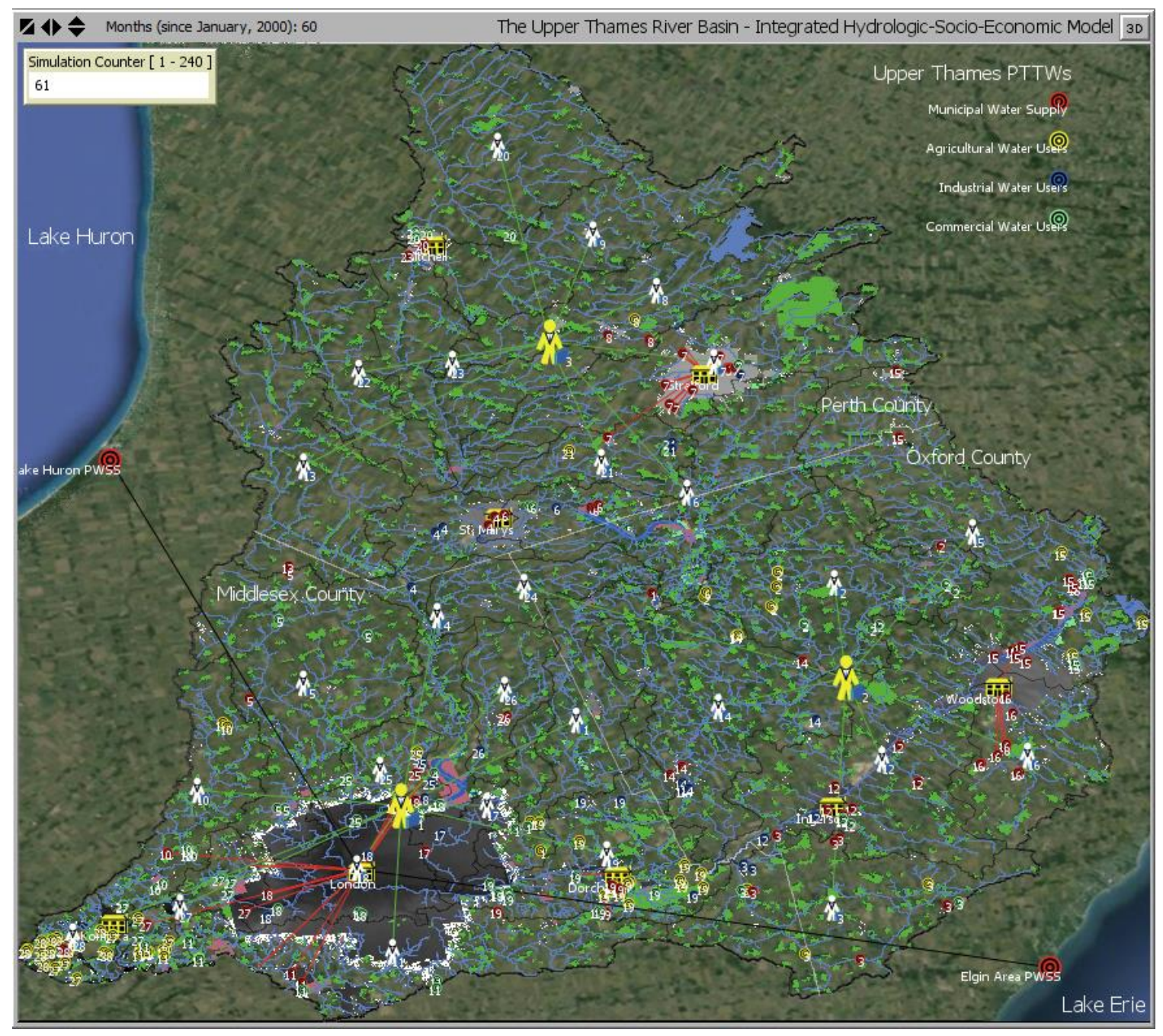

Figure 36: Dynamics of land use change and active water users in the Upper Thames River basin at time step 61 (January, 2005) 


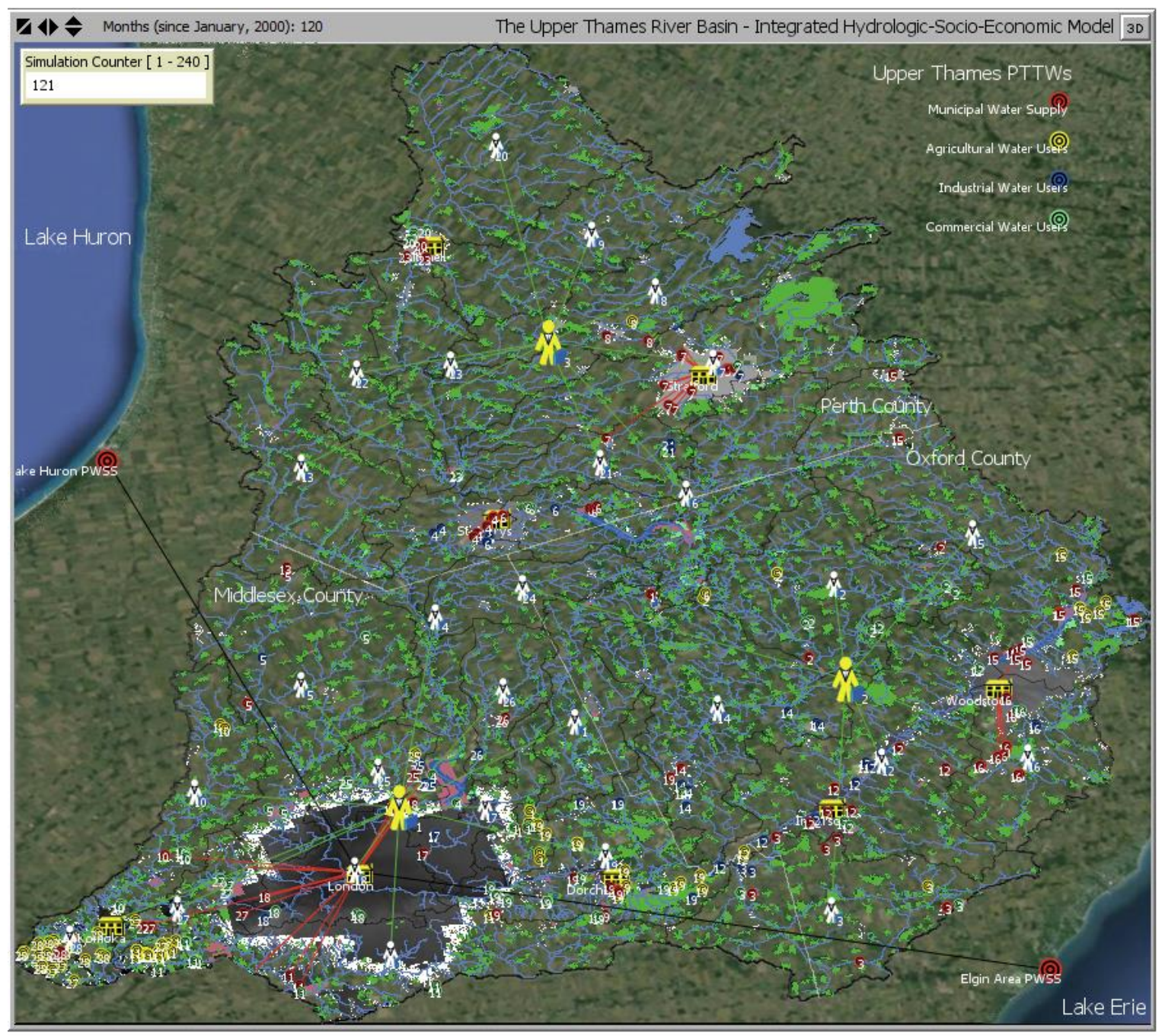

Figure 37: Dynamics of land use change and active water users in the Upper Thames River basin at time step 121(January, 2010) 


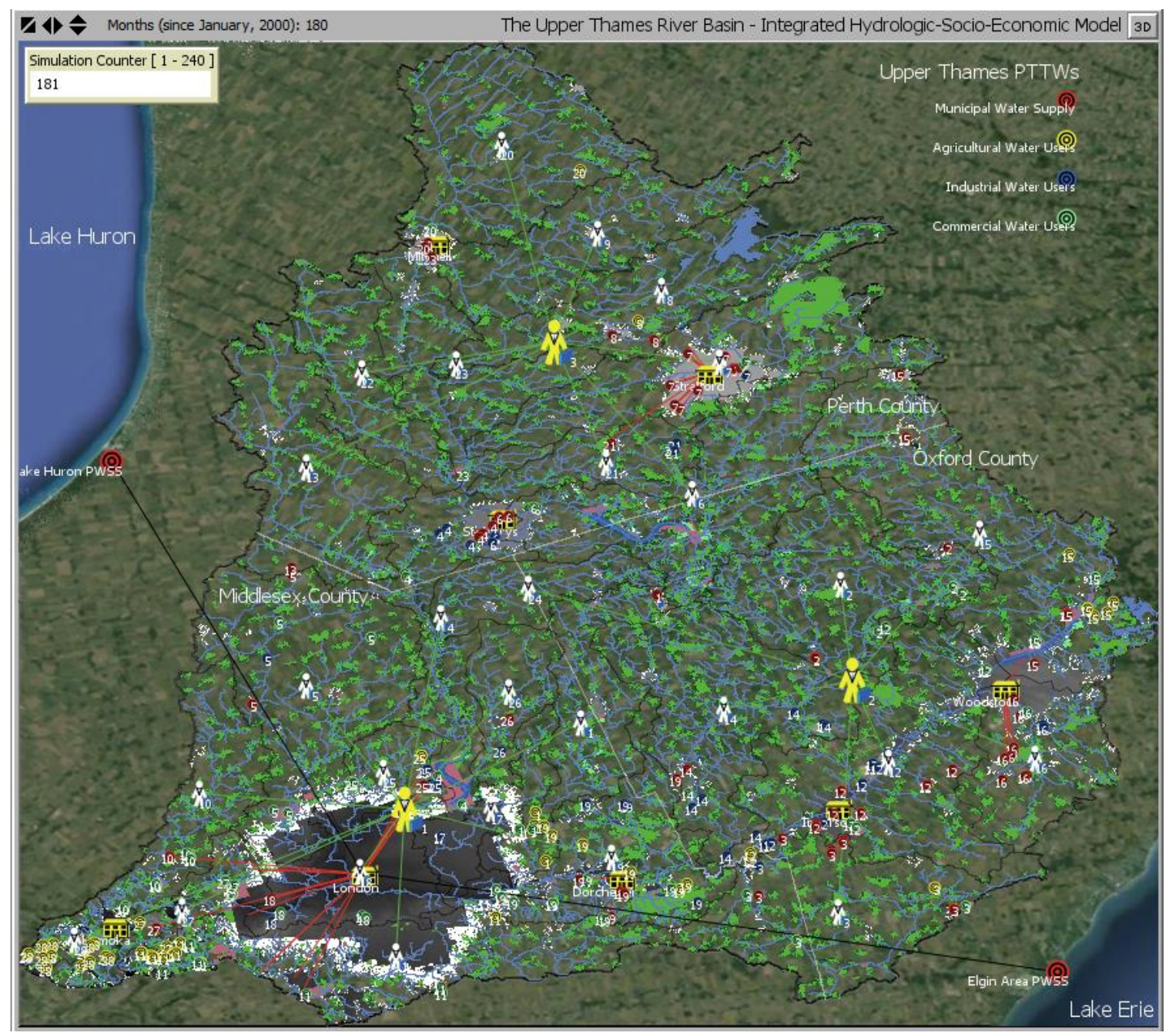

Figure 38: Dynamics of land use change and active water users in the Upper Thames River basin at time step 181 (January, 2015) 


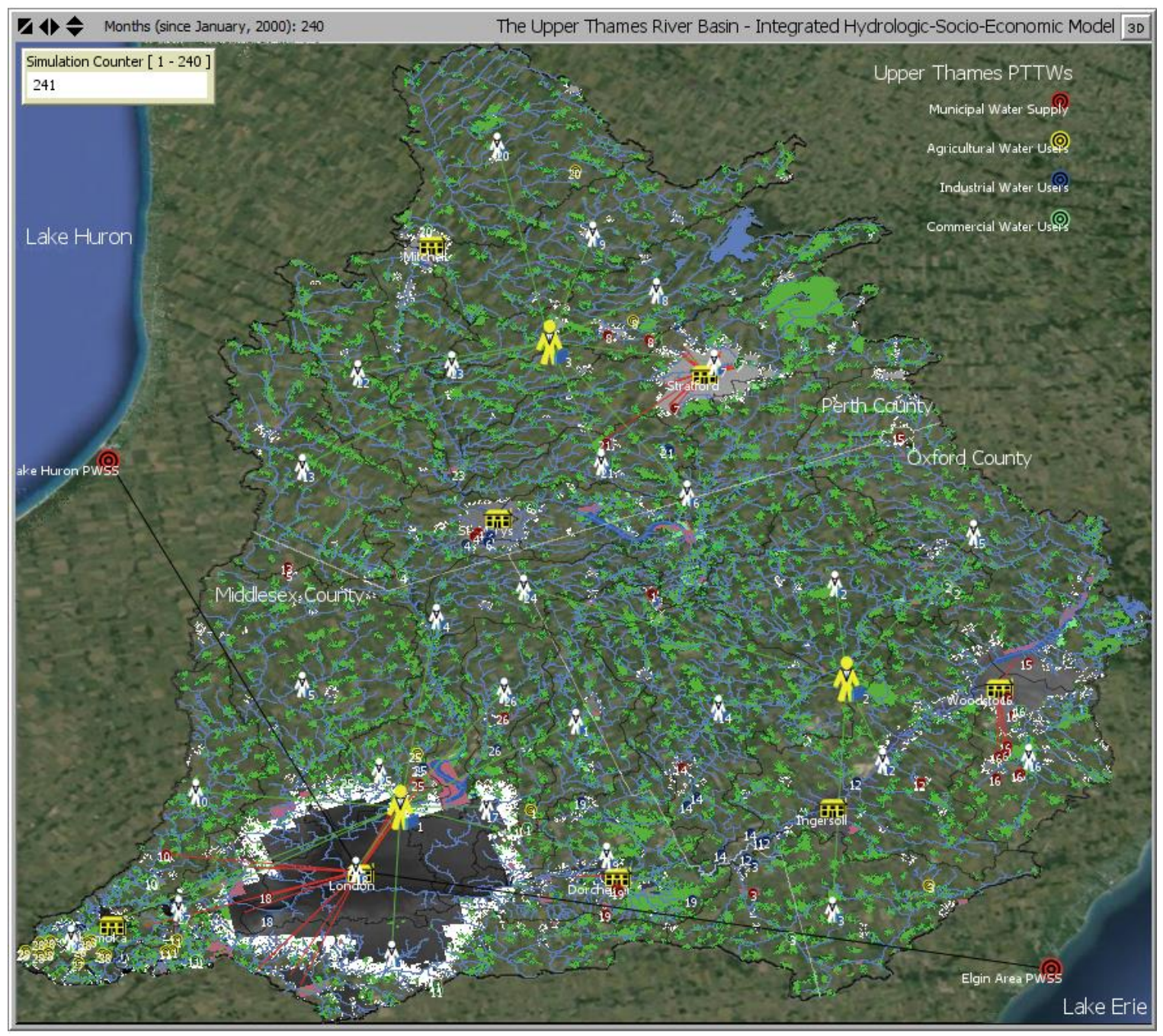

Figure 39: Dynamics of land use change and active water users in the Upper Thames River basin at time step 241 (January, 2020) 


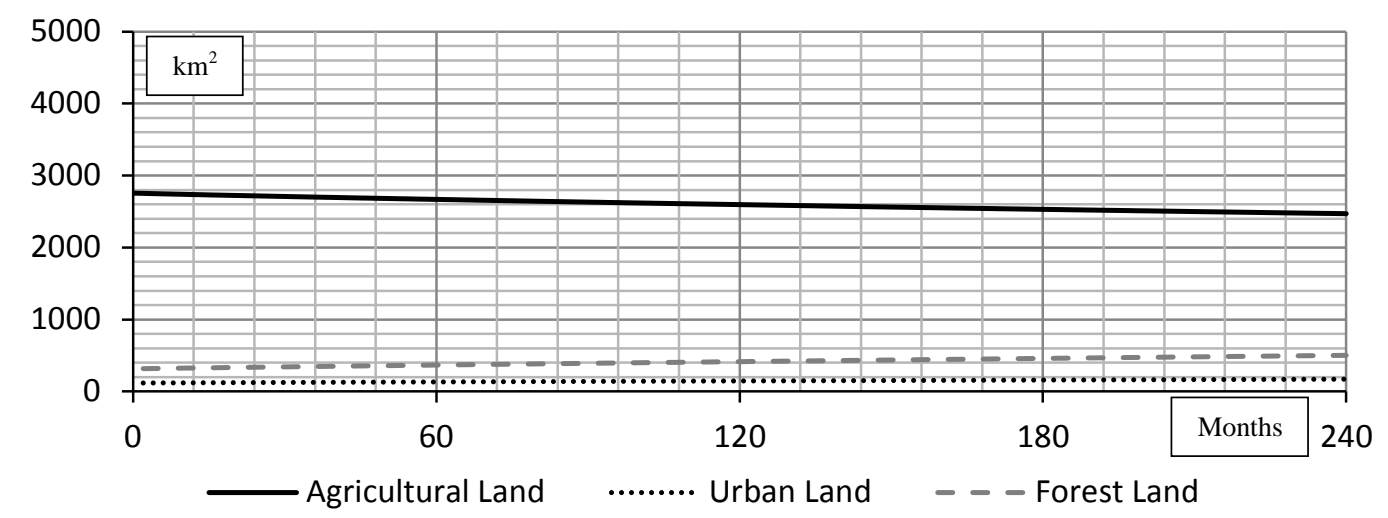

Figure 40: Types of land use in the UTRB

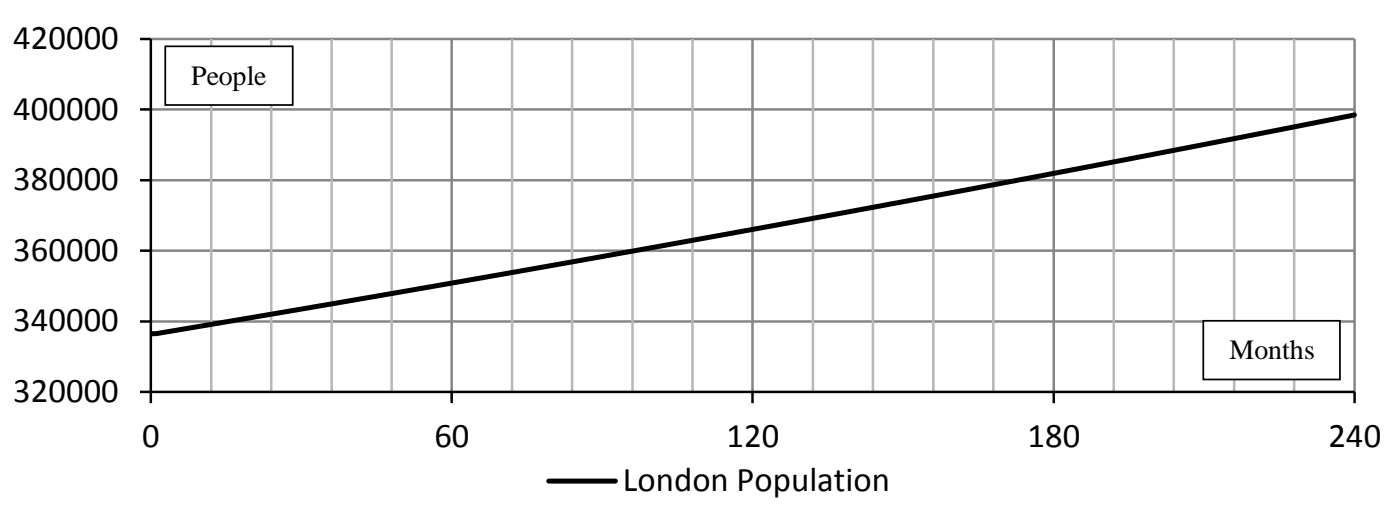

Figure 41: Population dynamics for the City of London

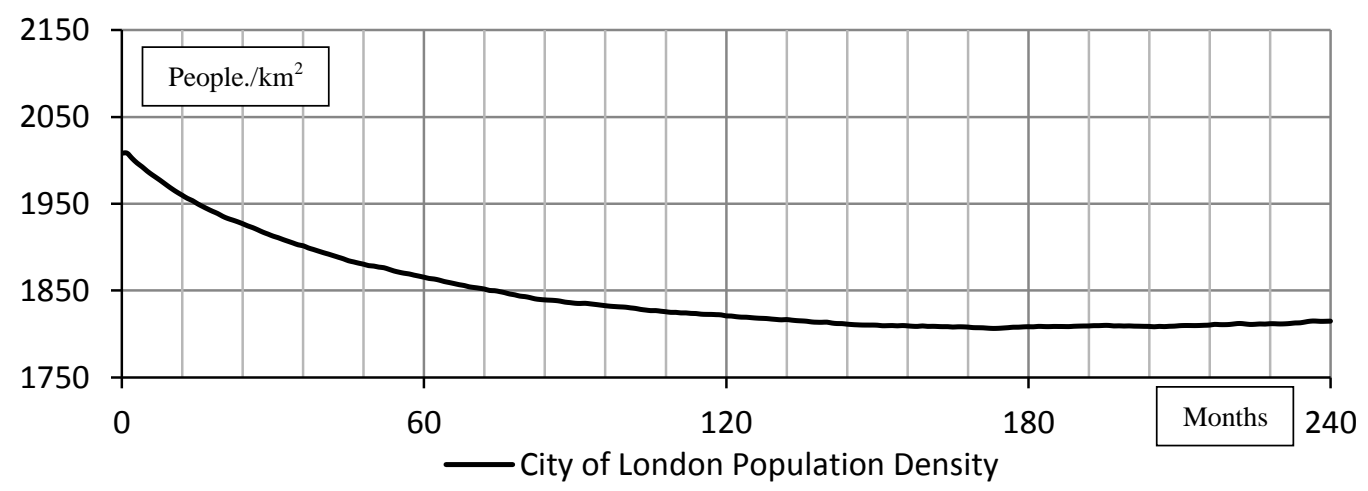

Figure 42: Population density for the City of London 


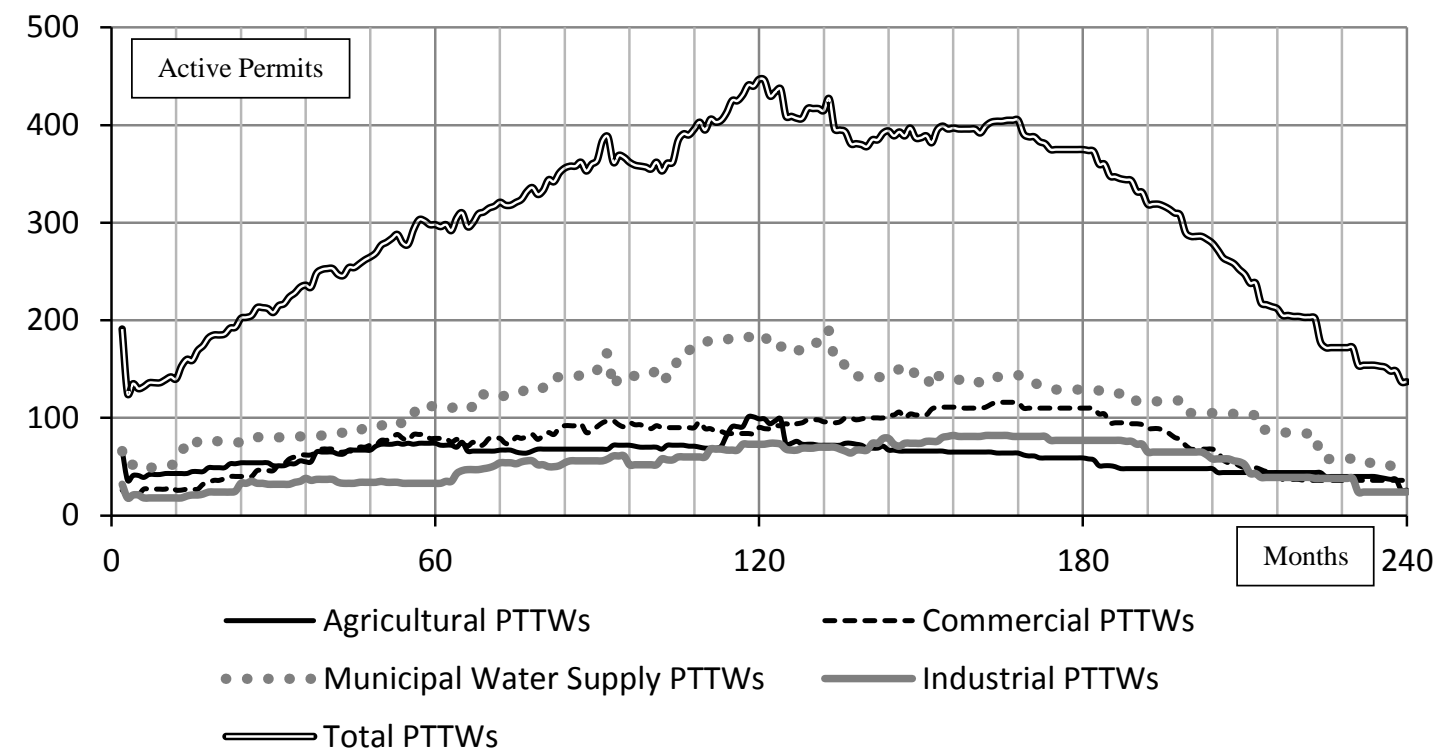

Figure 43: Active Permits to Take Water (PTTW)

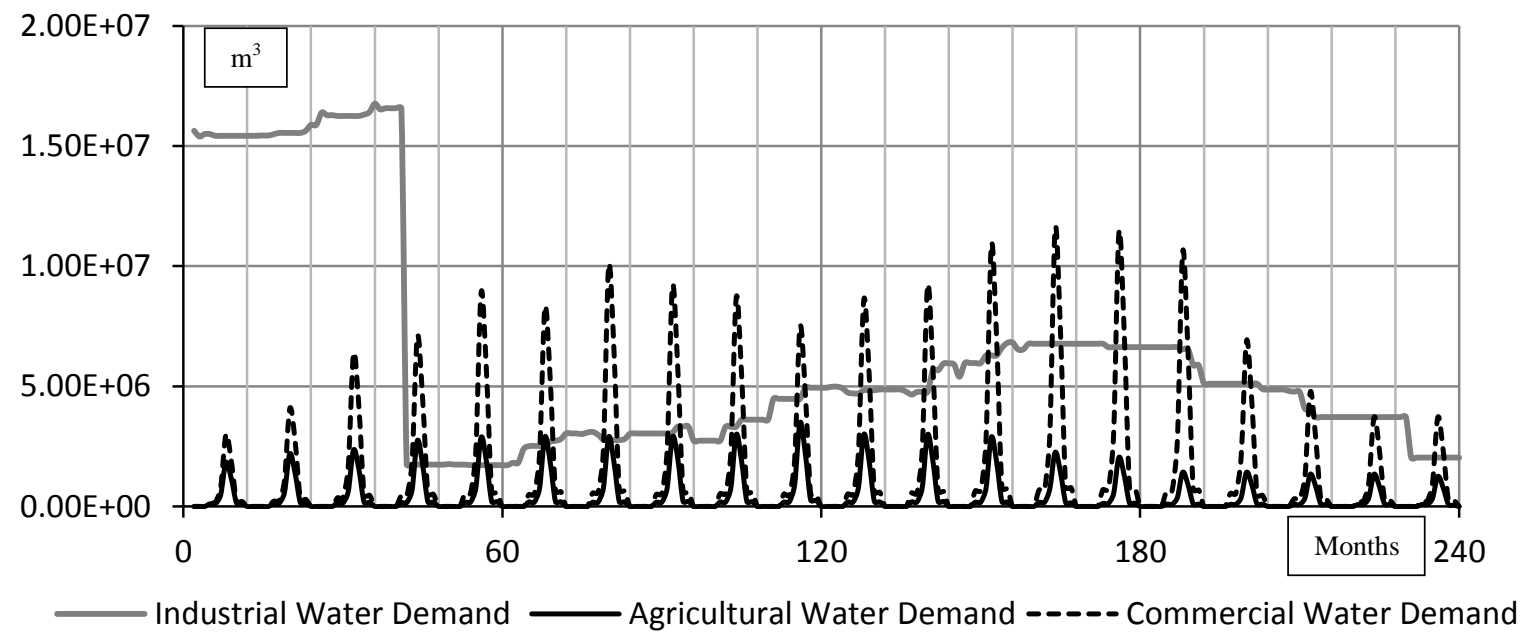

Figure 44: Monthly water demand by sector 


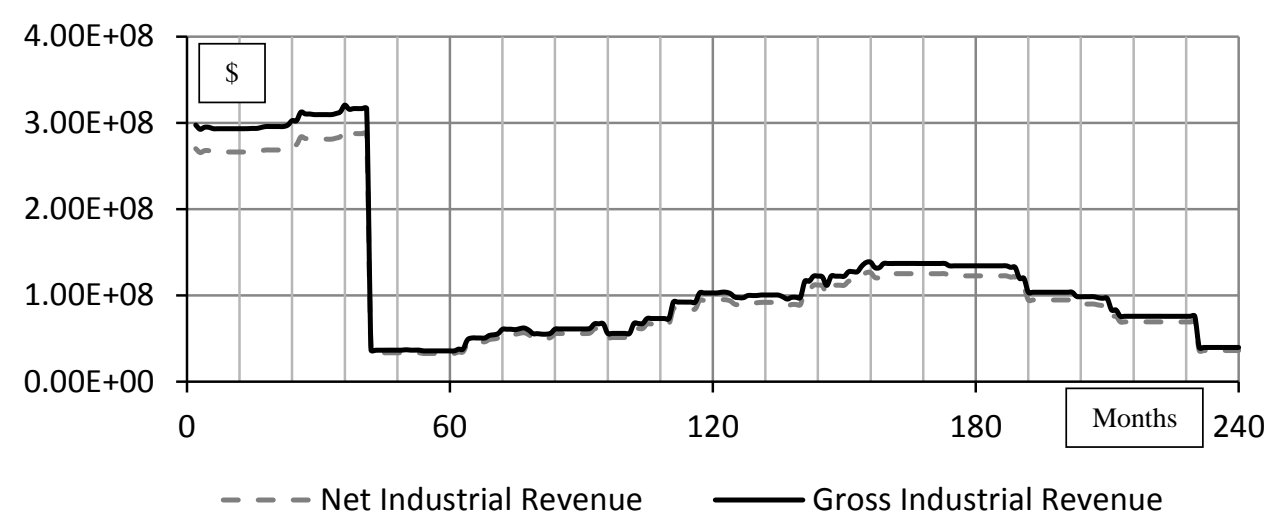

Figure 45: Industrial economic revenue based on the individual water demand

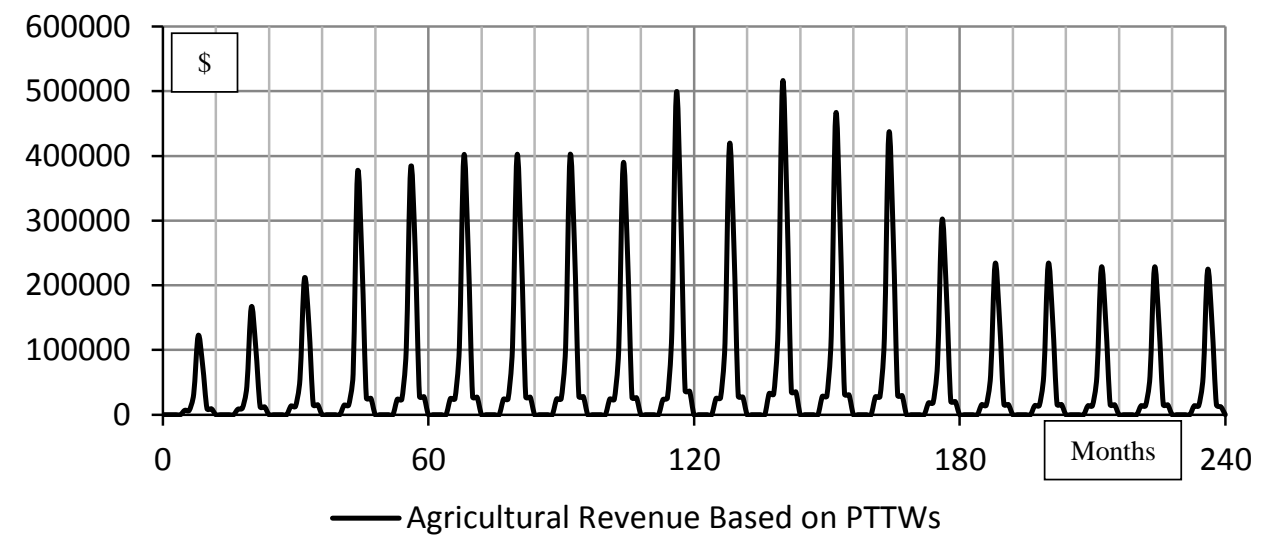

Figure 46: Agricultural economic revenue based on the individual water demand

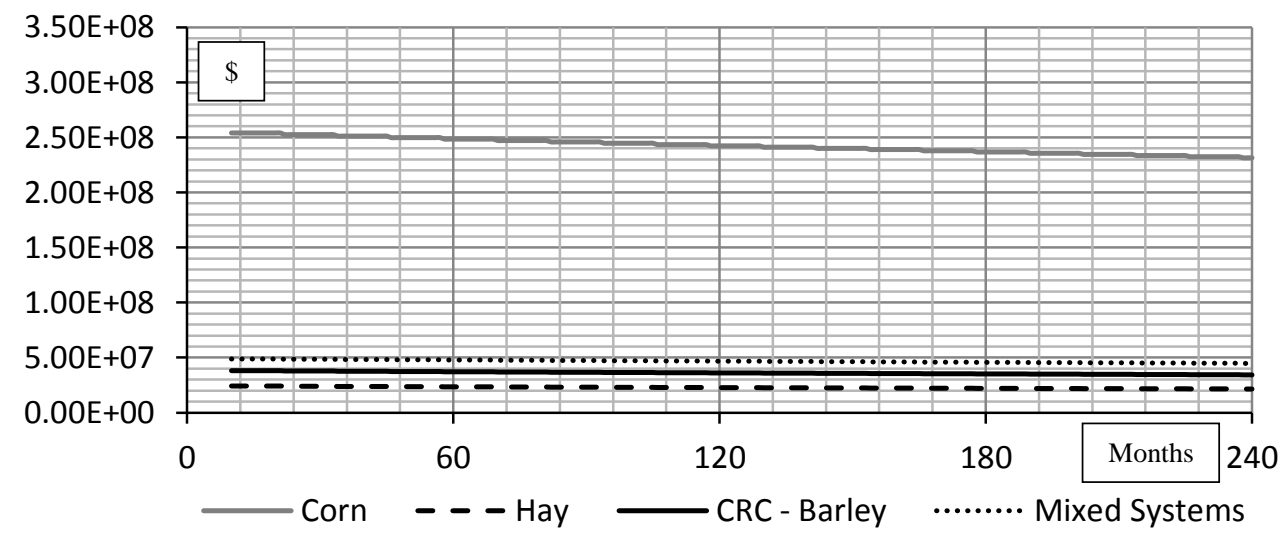

Figure 47: Agricultural economic revenue based on the farmed area 


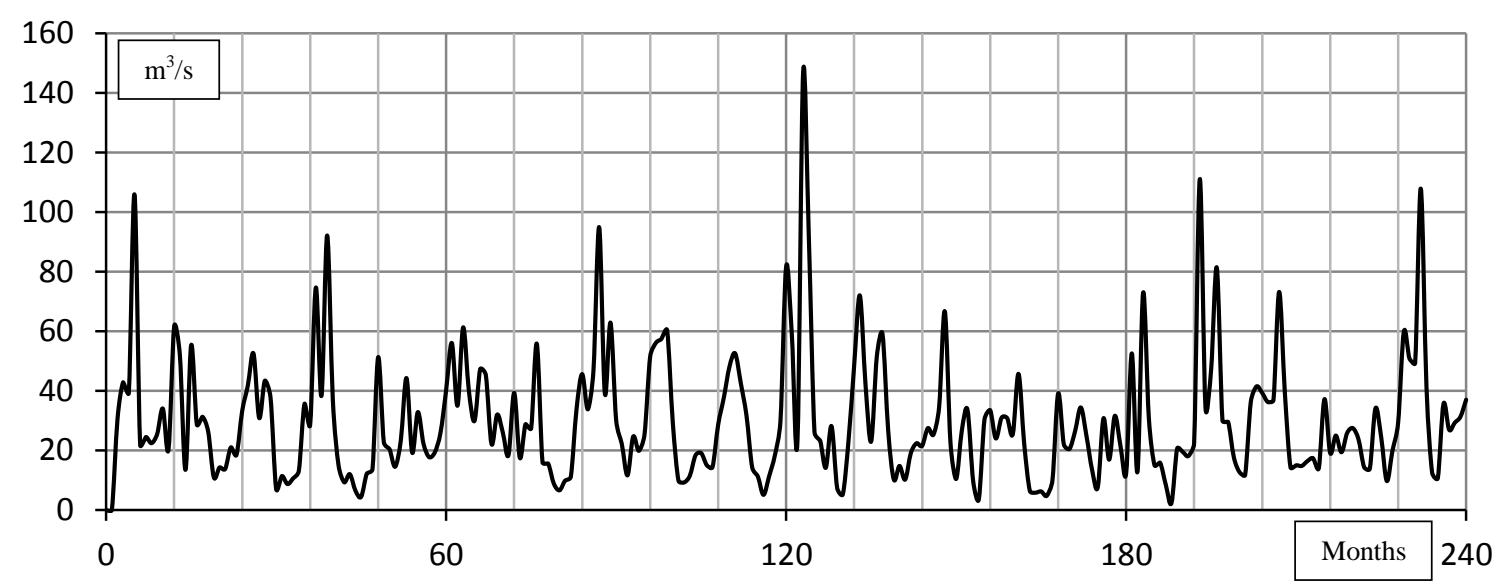

(a) Historic Climate Conditions

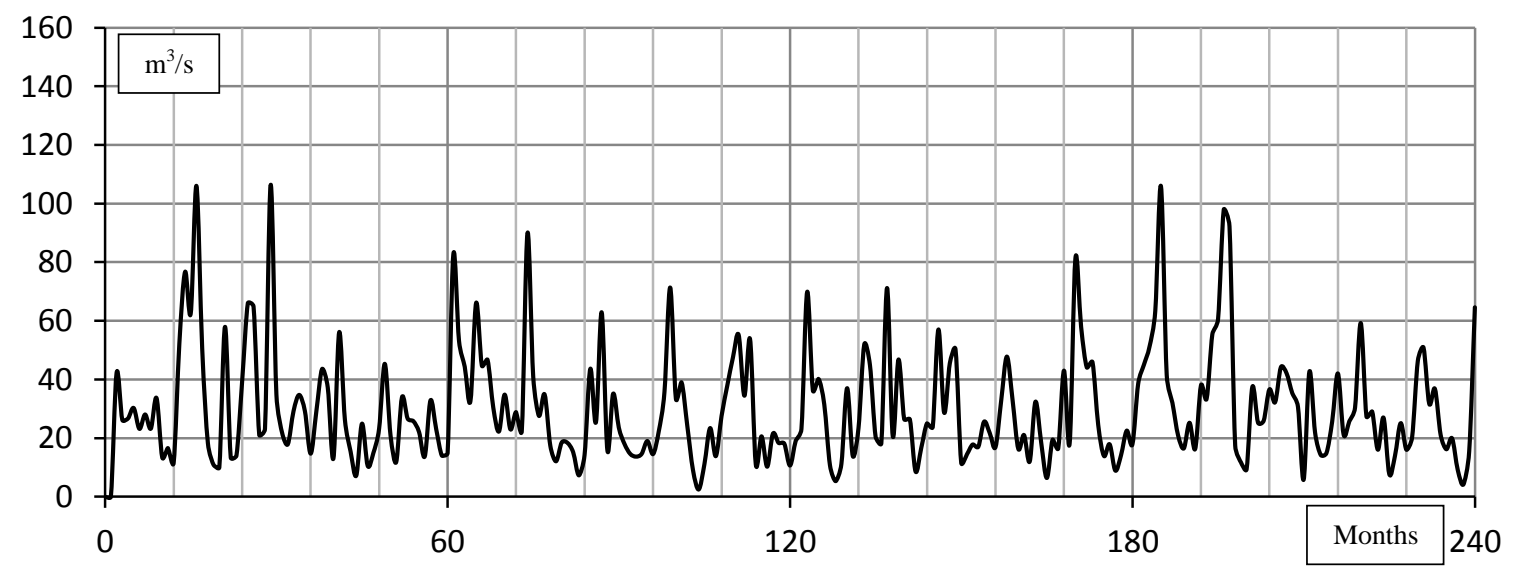

(b) Wet Climate Conditions

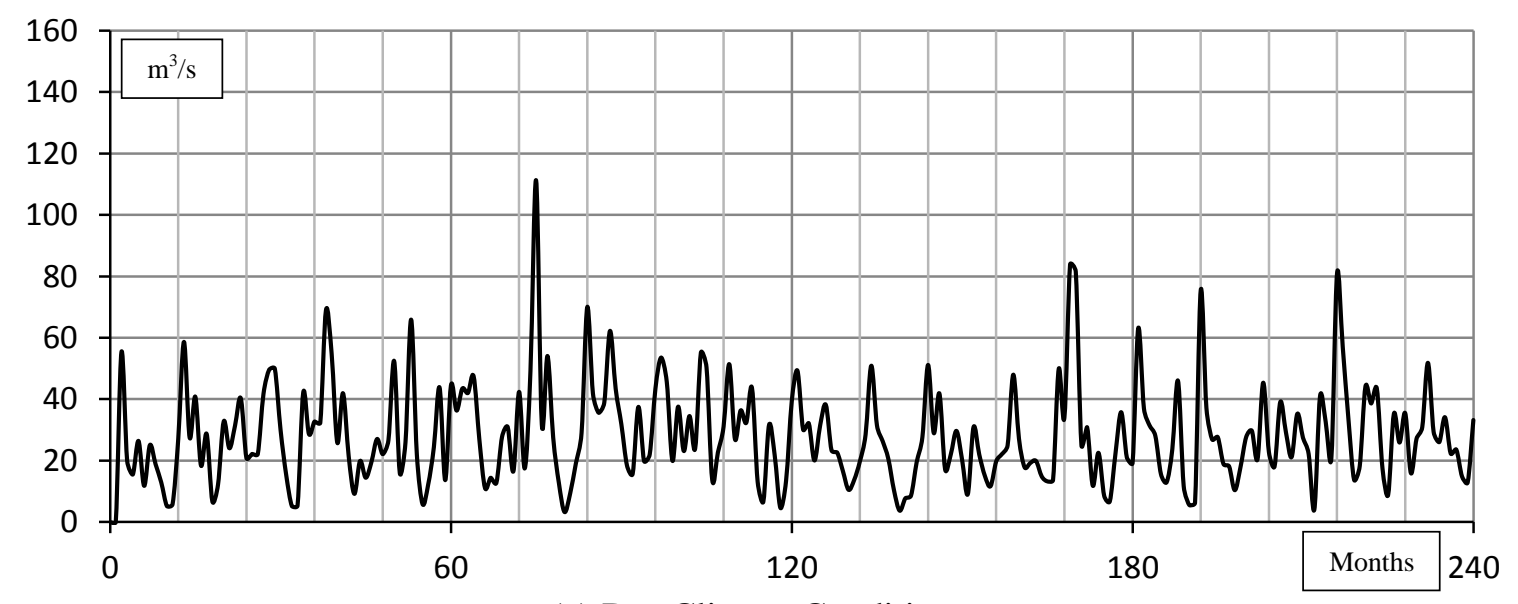

(c) Dry Climate Conditions

Figure 48: River Thames median monthly flow rates at Byron for three climate scenarios $(\mathrm{a}, \mathrm{b}, \mathrm{c})$ 


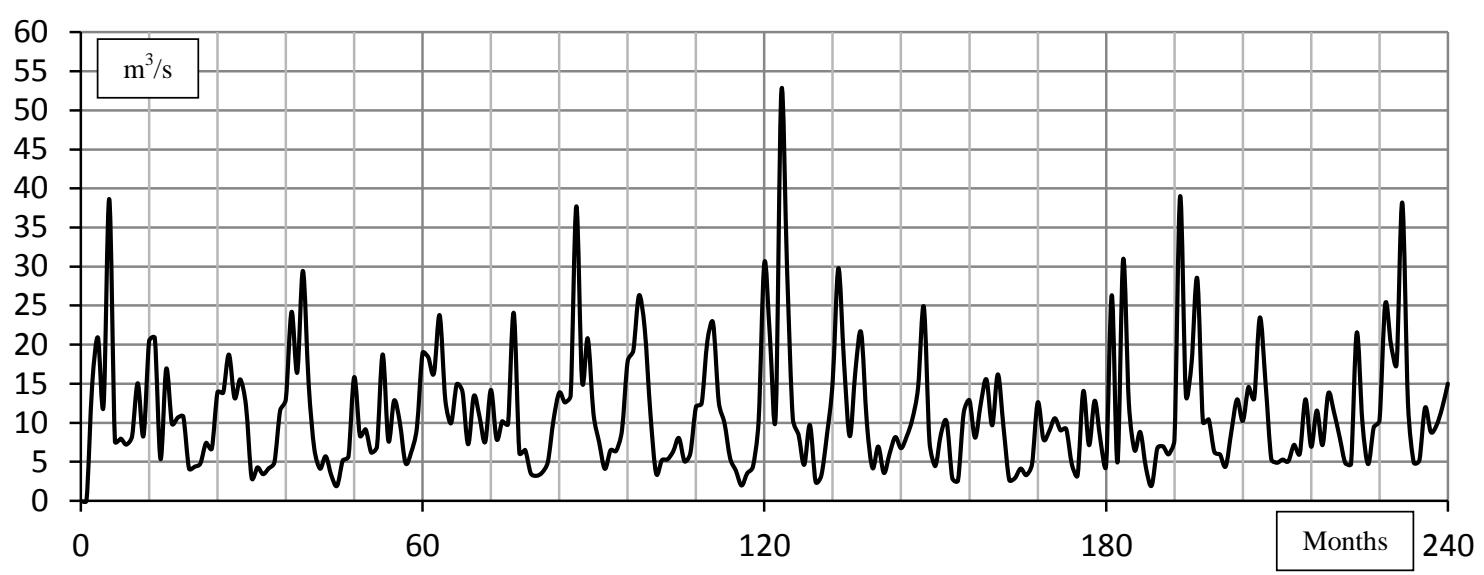

(a) Historic Climate Conditions

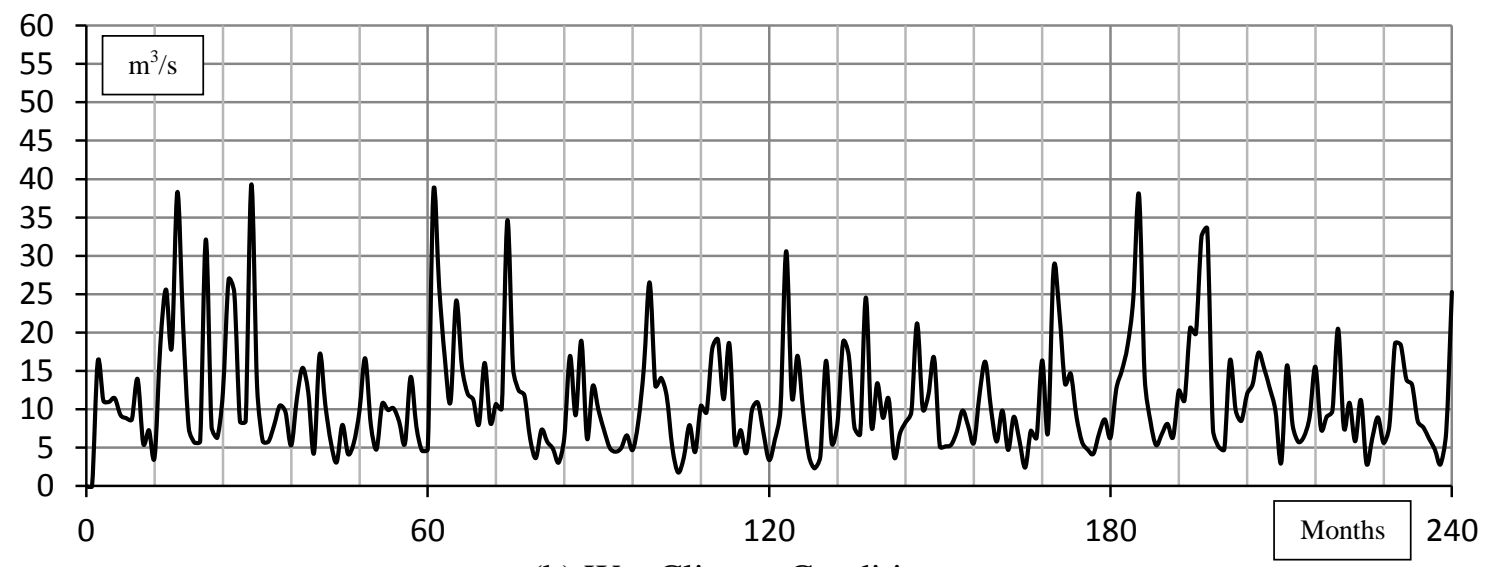

(b) Wet Climate Conditions

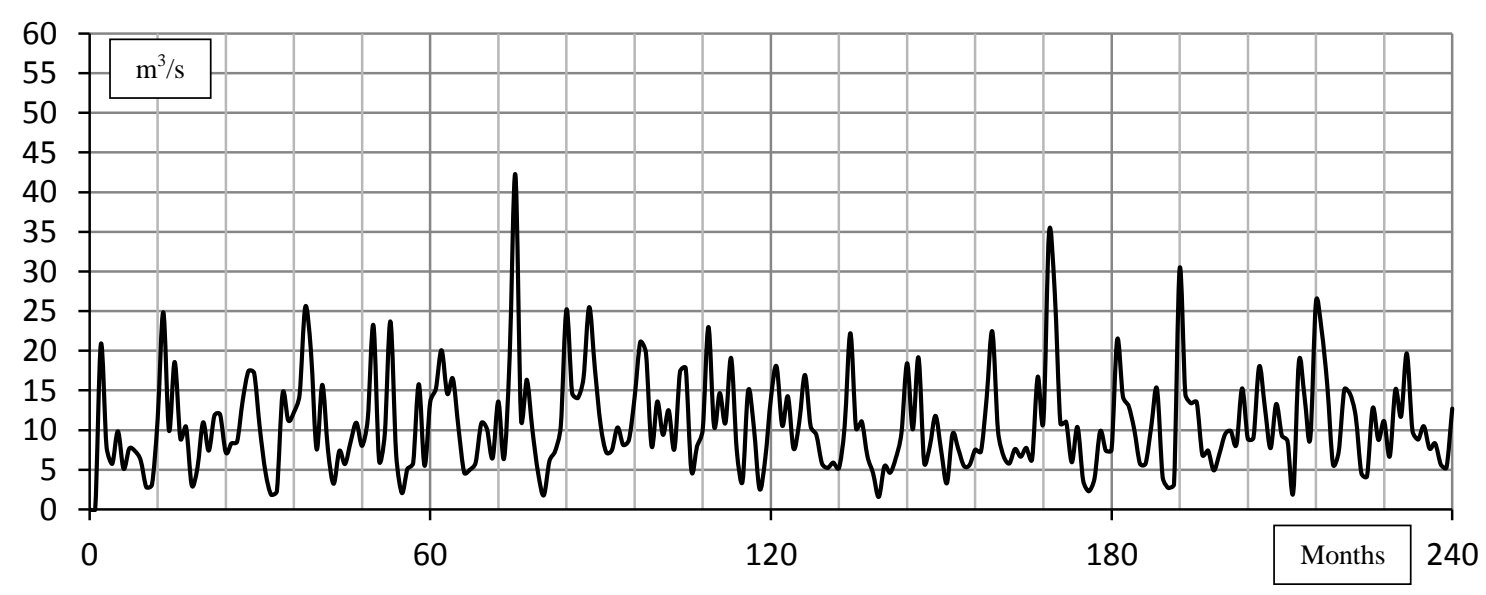

(c) Dry Climate Conditions

Figure 49: River Thames median monthly flow rates at Ingersoll for three climate scenarios $(a, b, c)$ 


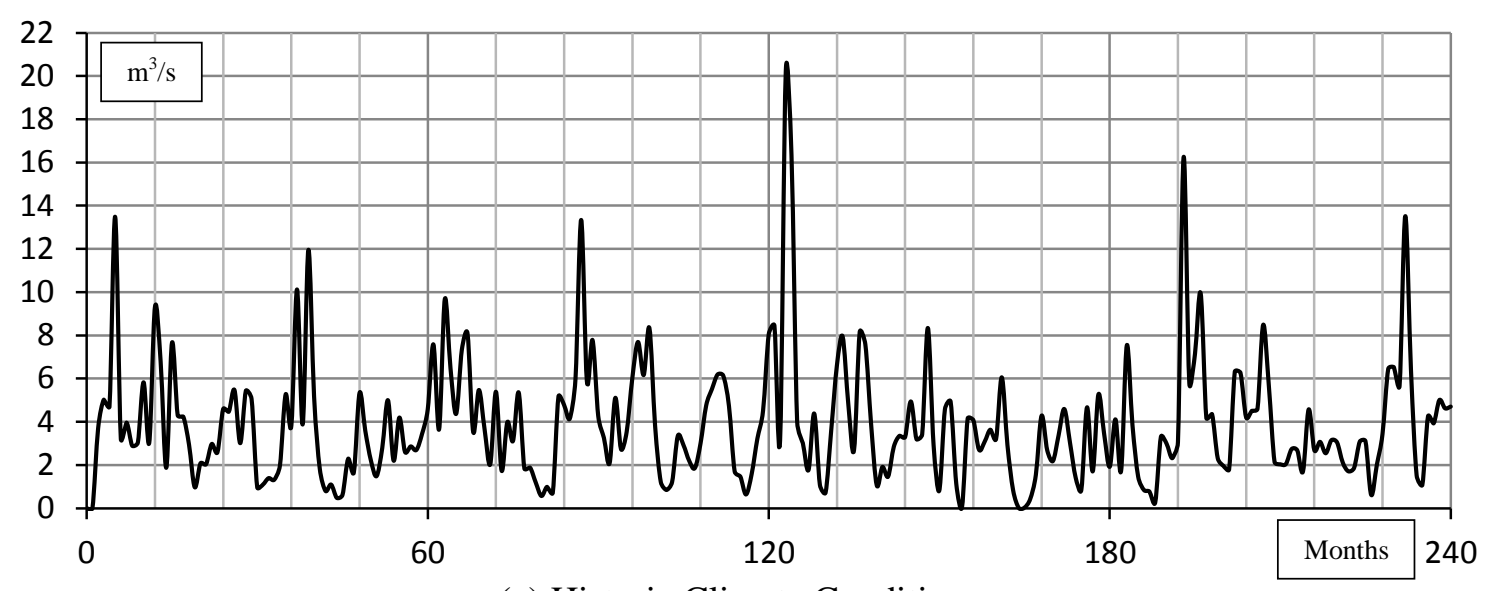

(a) Historic Climate Conditions

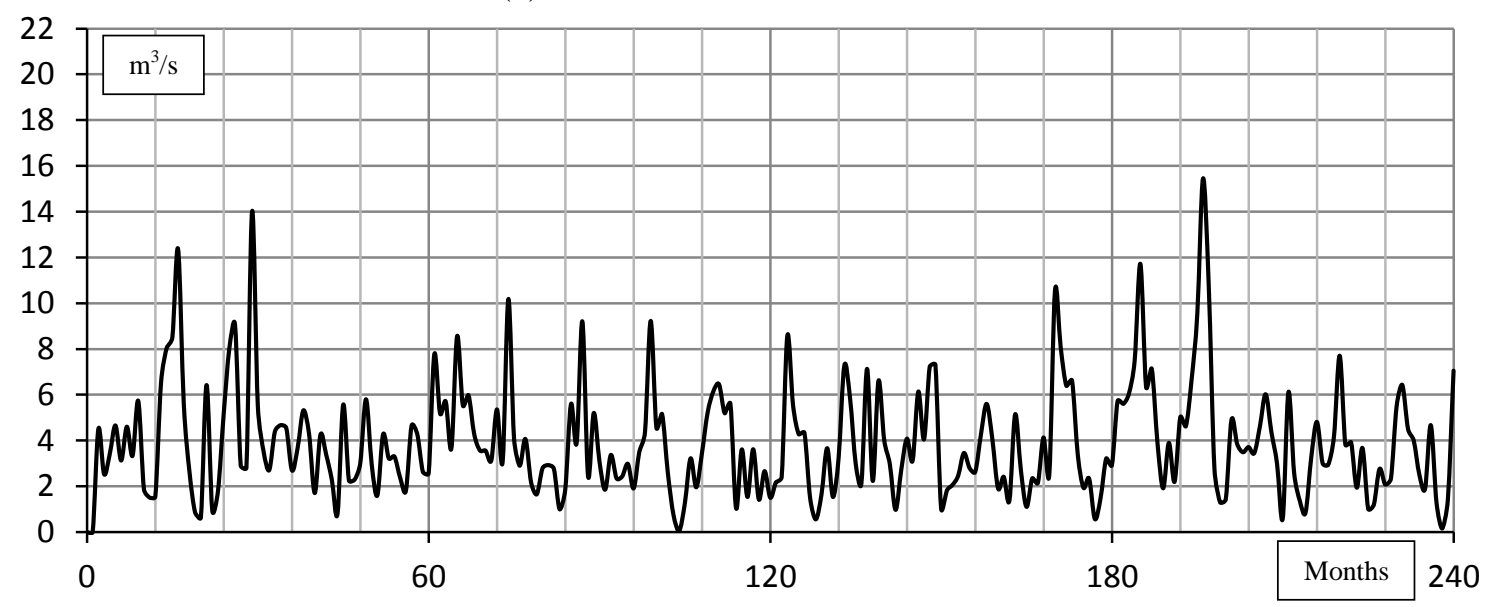

(b) Wet Climate Conditions

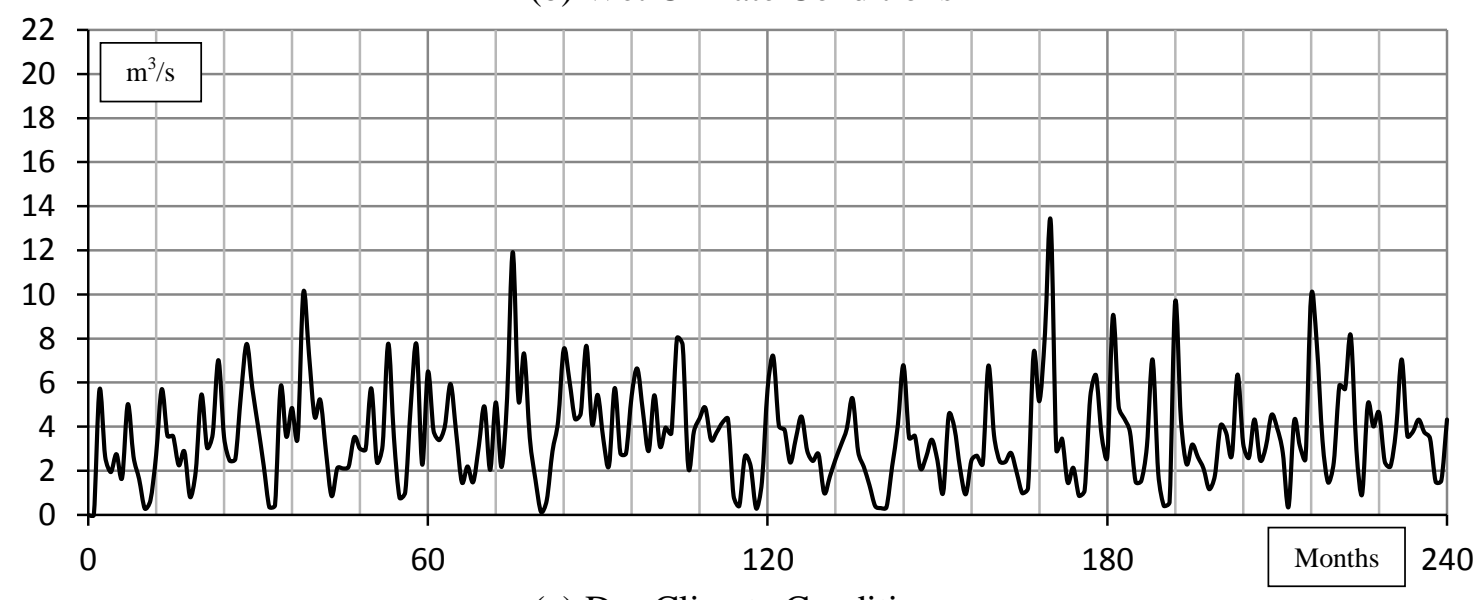

(c) Dry Climate Conditions

Figure 50: River Thames median monthly flow rates at St. Marys for three climate scenarios $(a, b, c)$ 


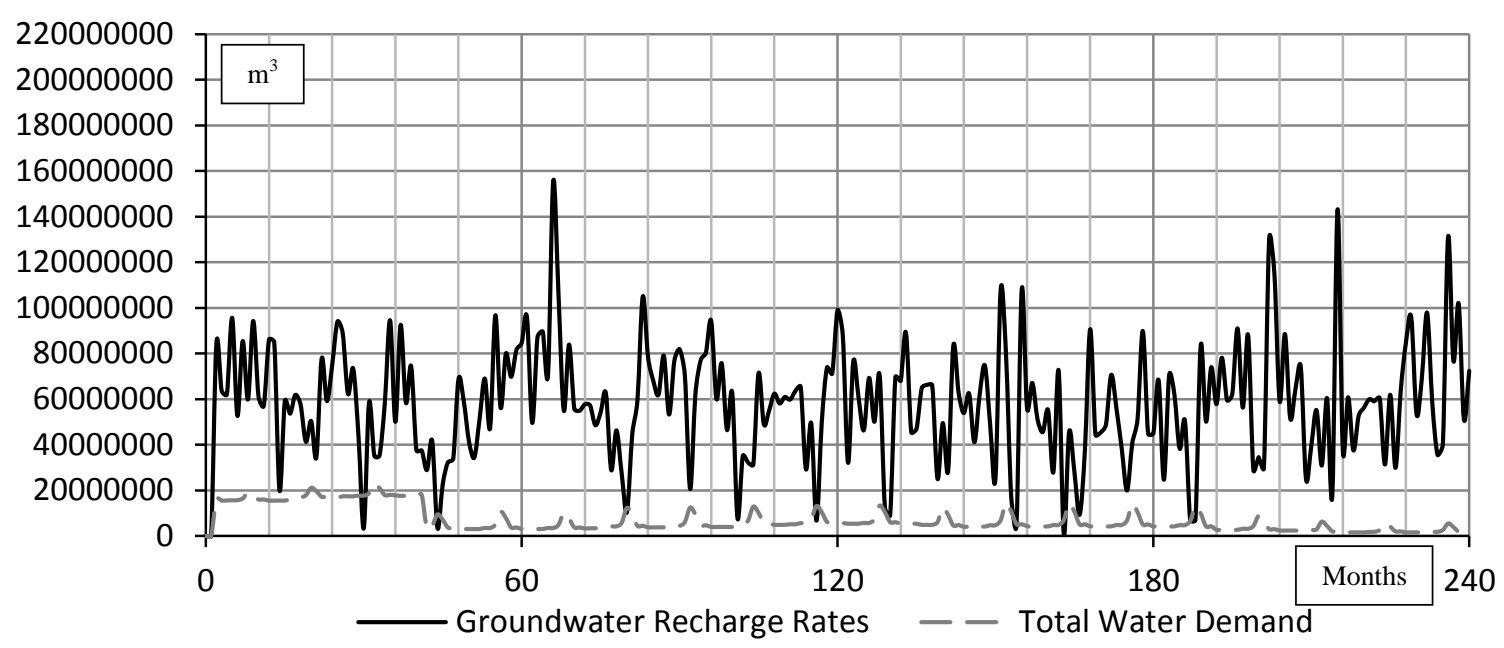

(a) Historic Climate Conditions

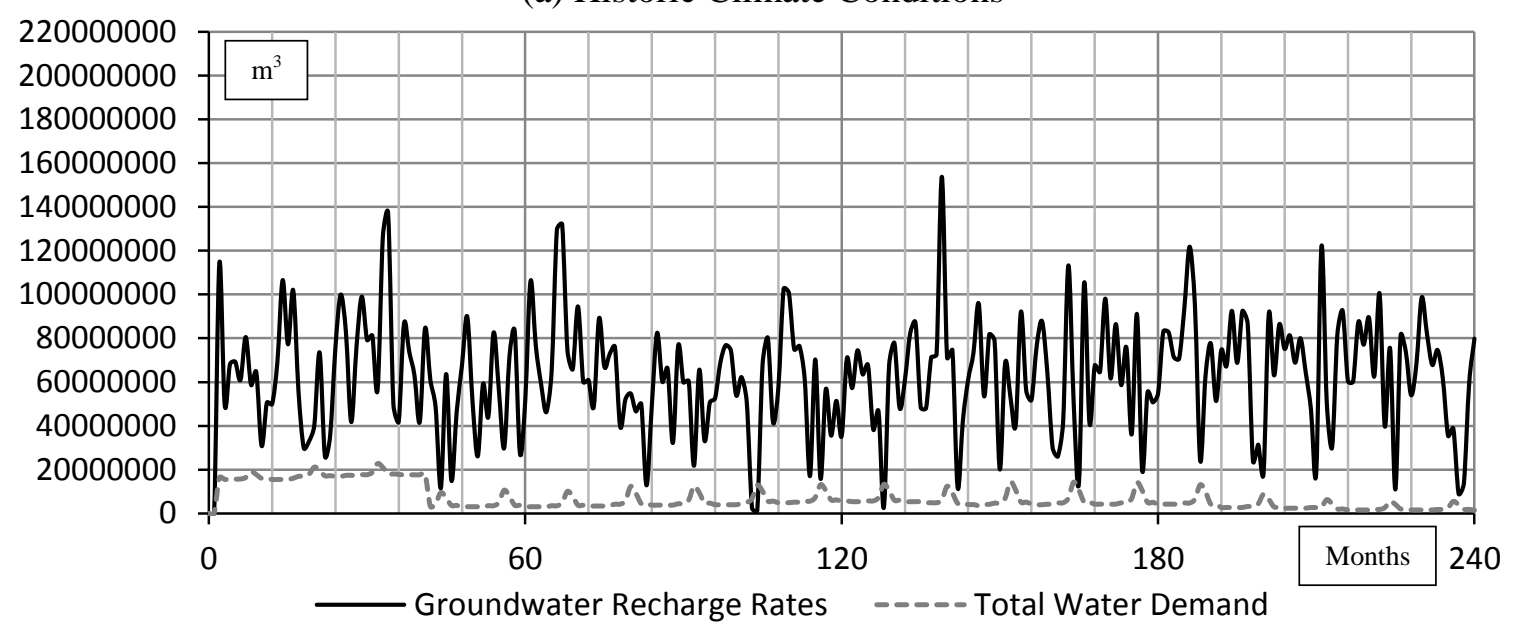

(b) Wet Climate Conditions

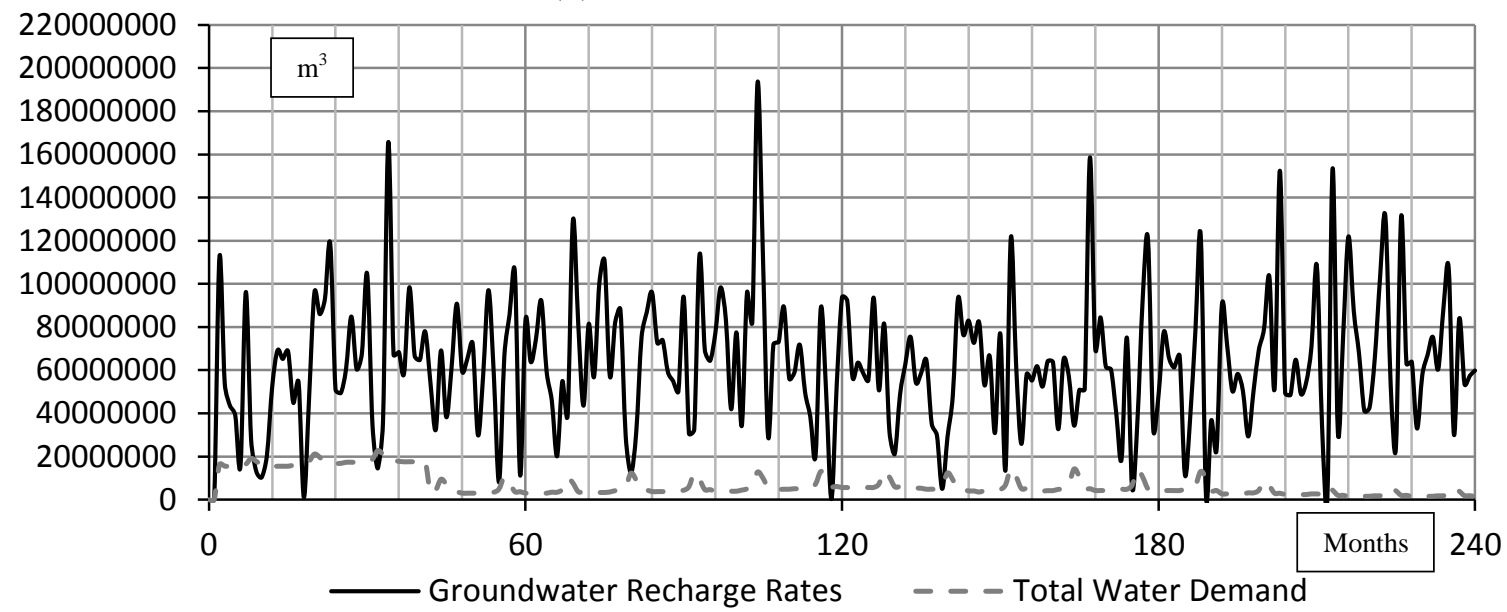

(c) Dry Climate Conditions

Figure 51: Middlesex County groundwater recharge rates and total water demand for three climate scenarios $(a, b, c)$ 


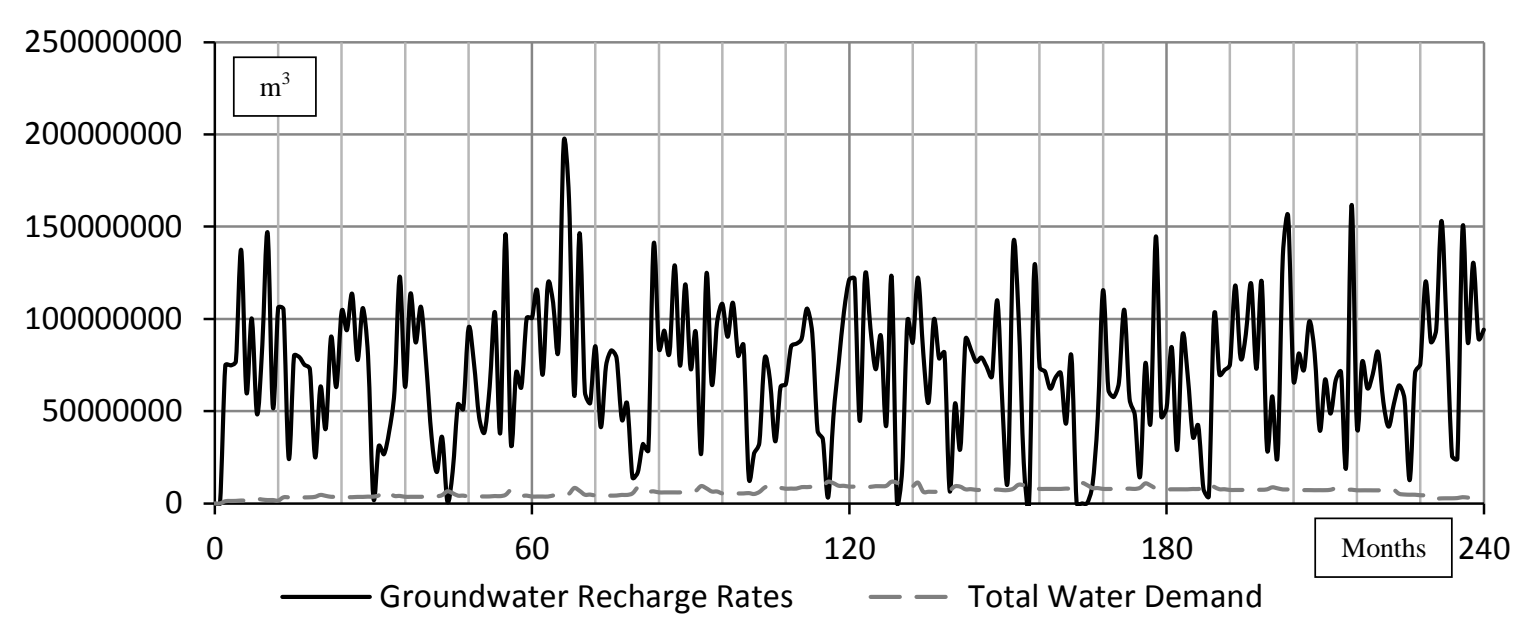

(a) Historic Climate Conditions

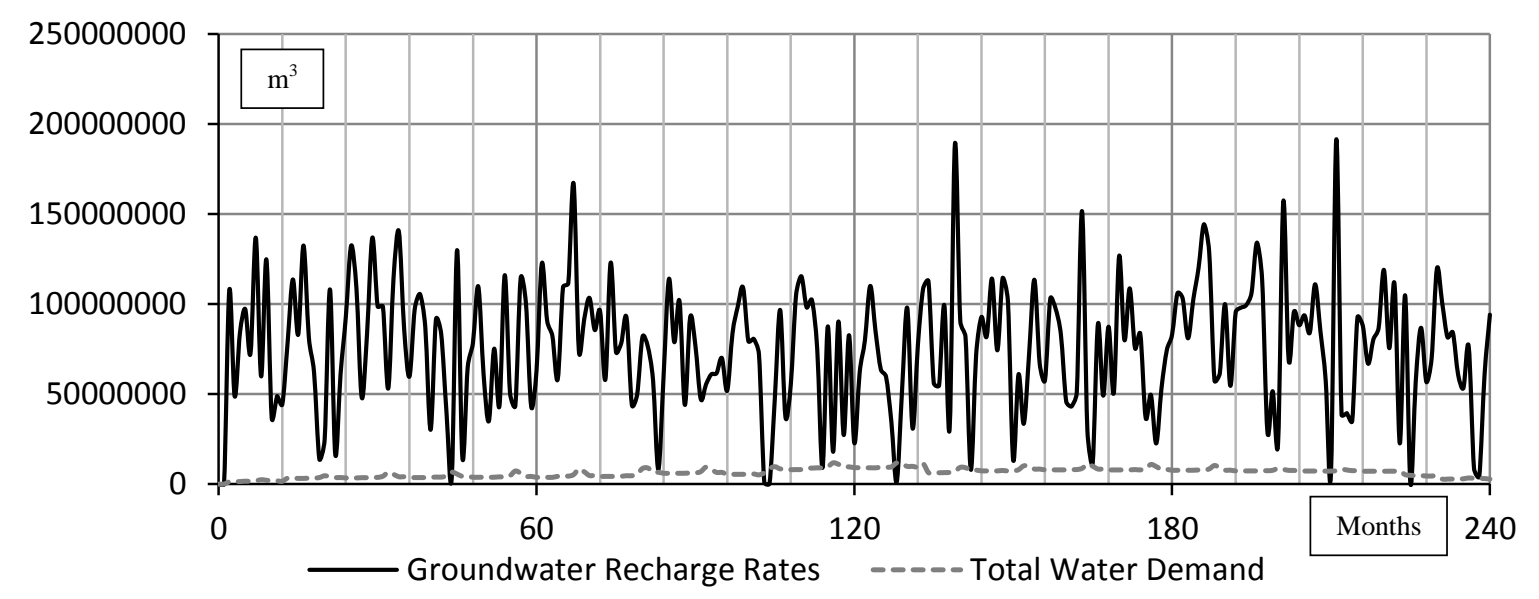

(b) Wet Climate Conditions

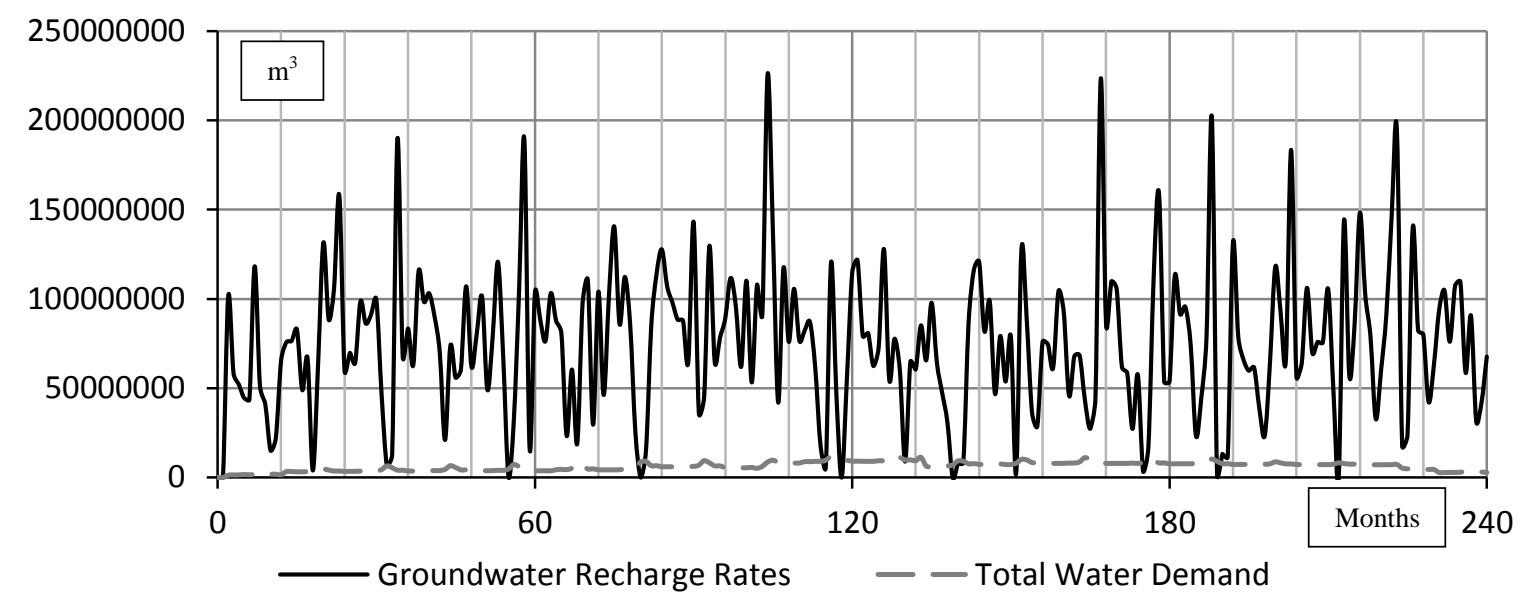

(c) Dry Climate Conditions

Figure 52: Oxford County groundwater recharge rates and total water demand for three climate scenarios $(a, b, c)$ 


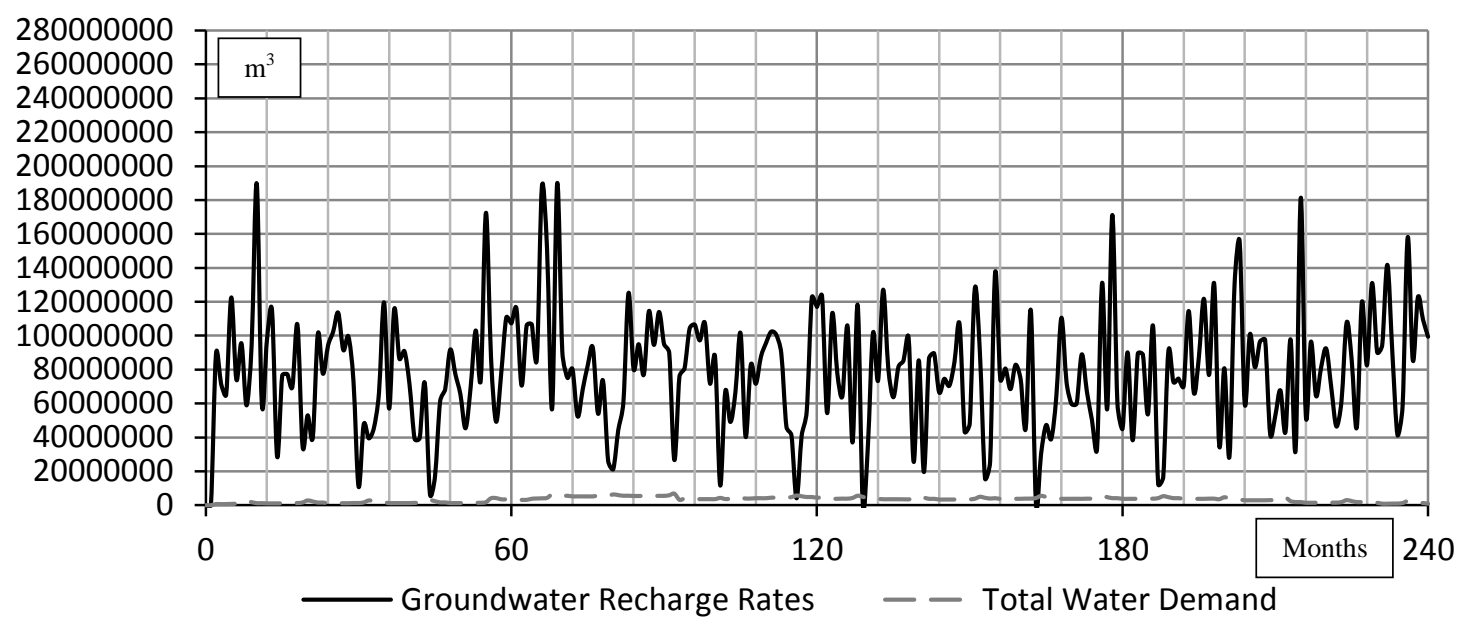

(a) Historic Climate Conditions

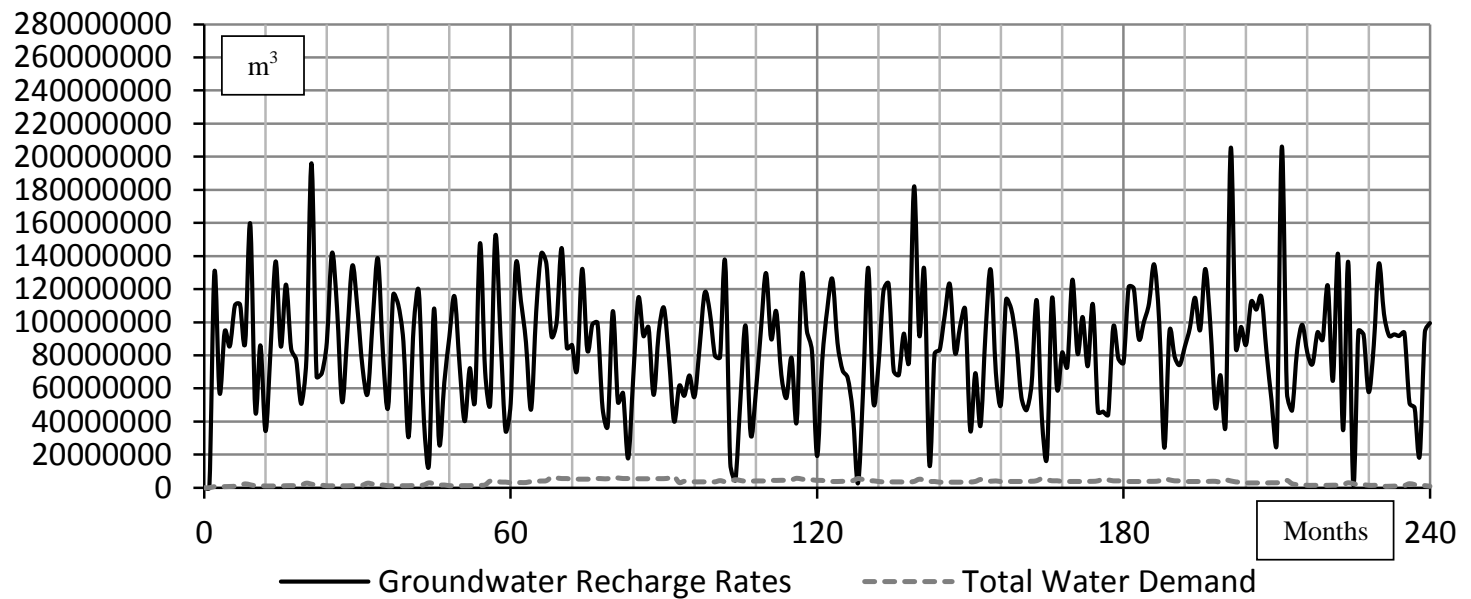

(b) Wet Climate Conditions

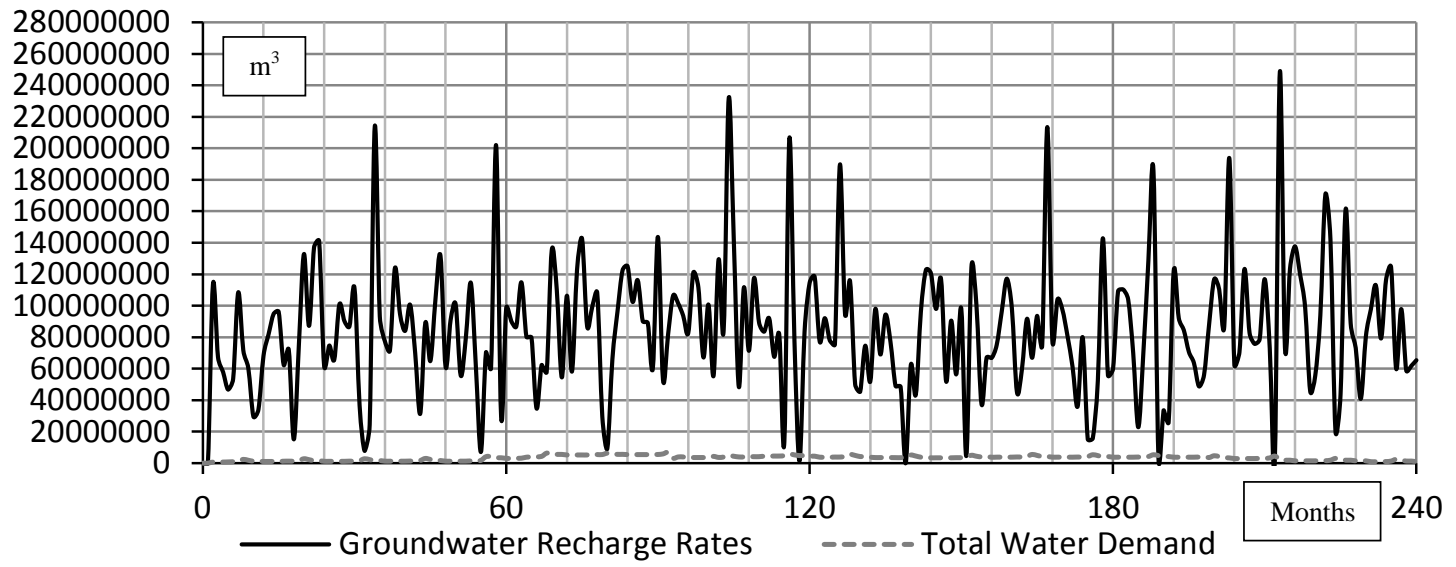

(c) Dry Climate Conditions

Figure 53: Perth County groundwater recharge rates and total water demand for three climate scenarios $(\mathrm{a}, \mathrm{b}, \mathrm{c})$ 


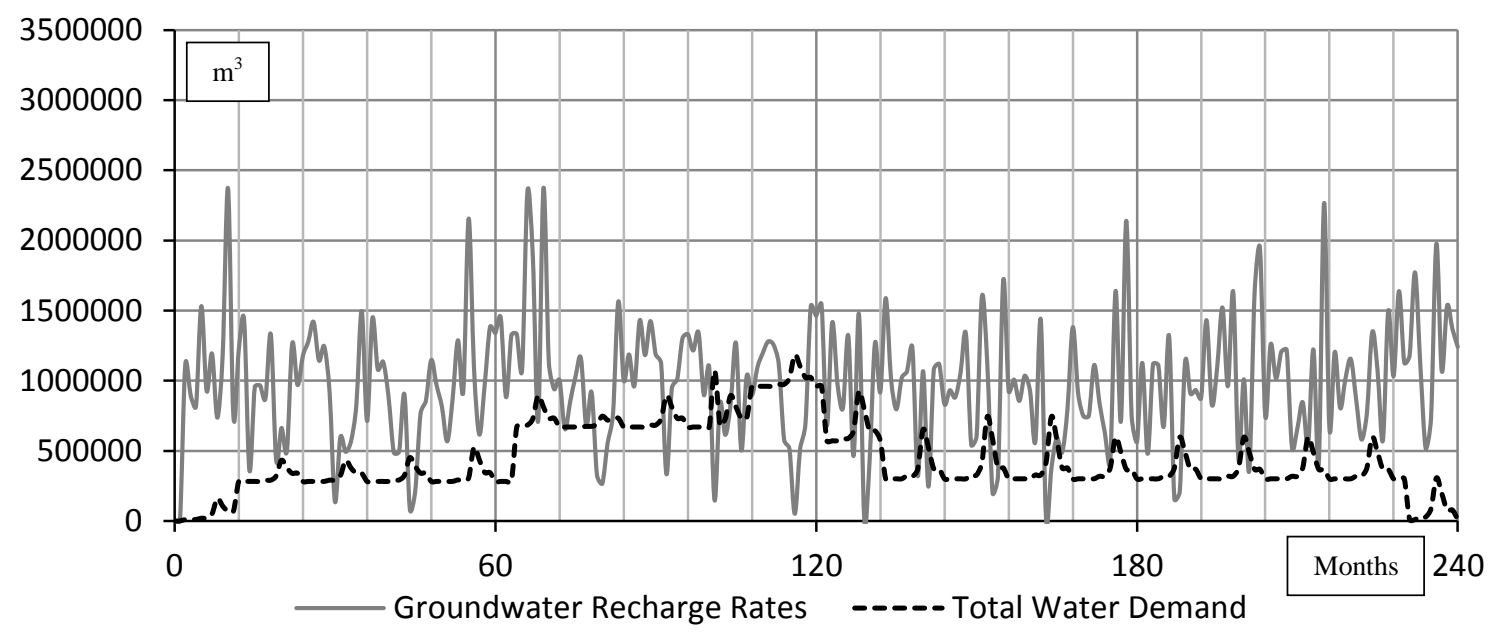

(a) Historic Climate Conditions

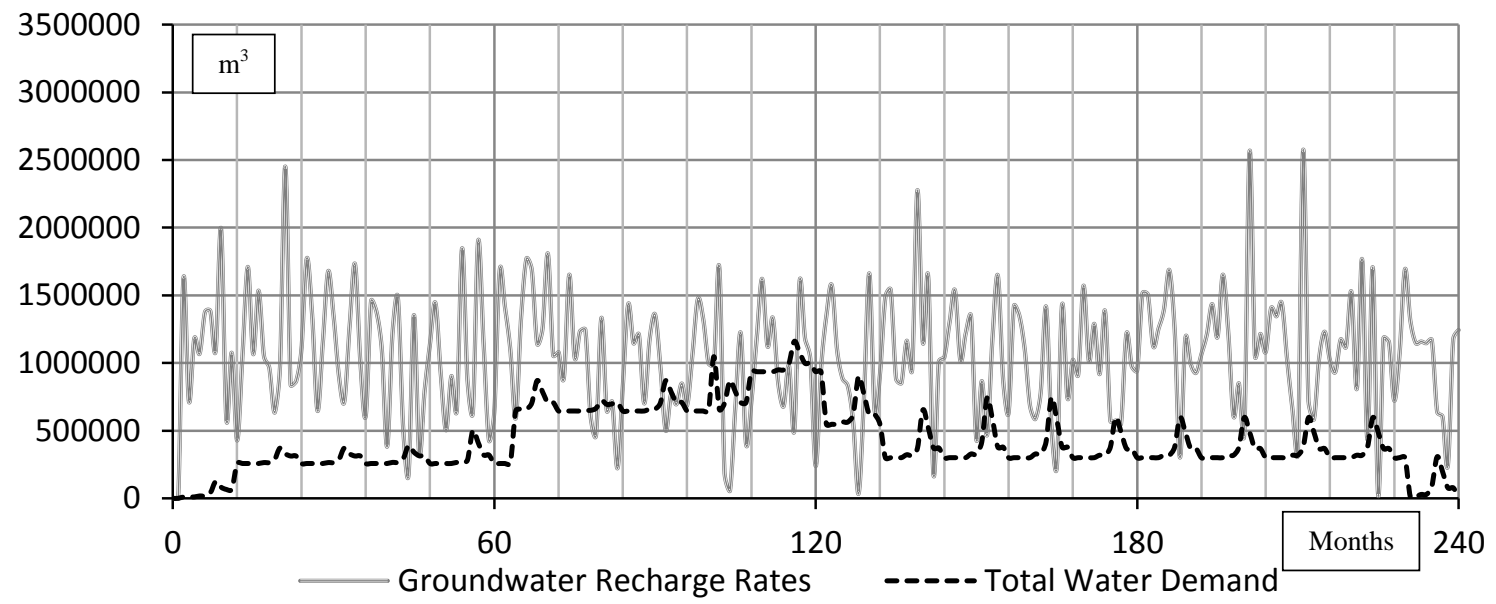

(b) Wet Climate Conditions

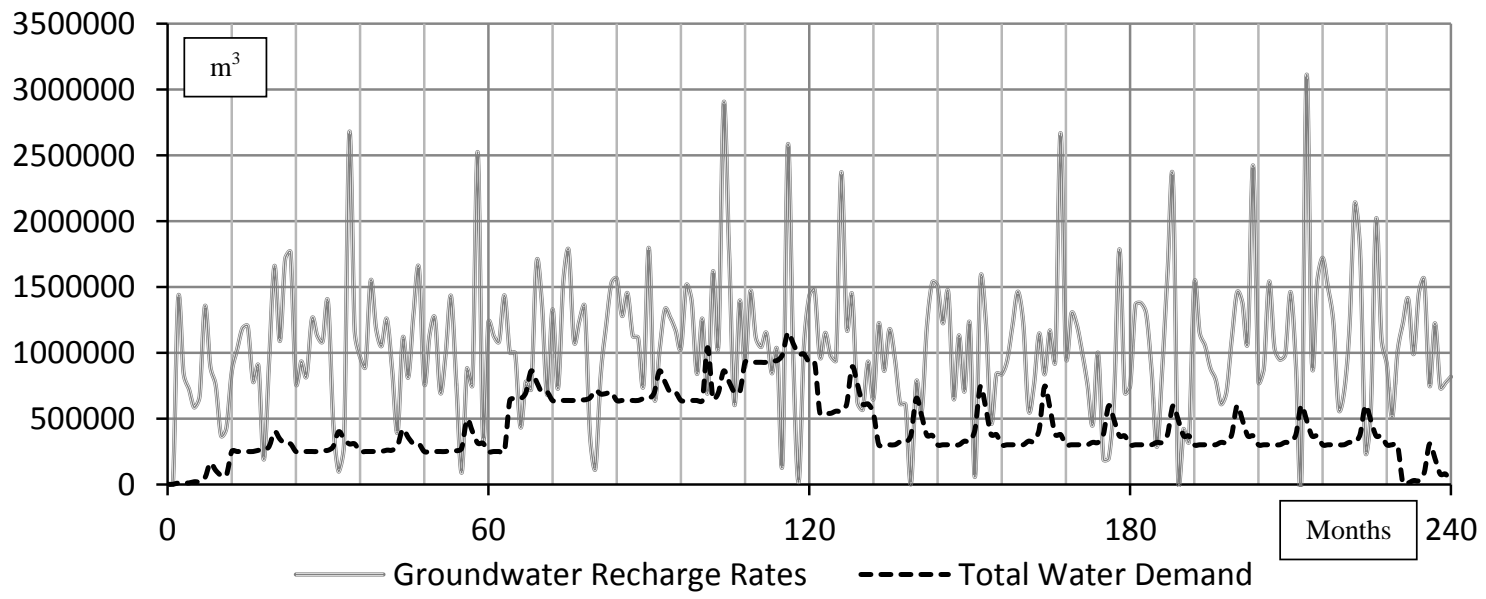

(c) Dry Climate Conditions

Figure 54: Middle Thames (sub-basin 14) groundwater recharge rates and total water demand for three climate scenarios $(\mathrm{a}, \mathrm{b}, \mathrm{c})$ 


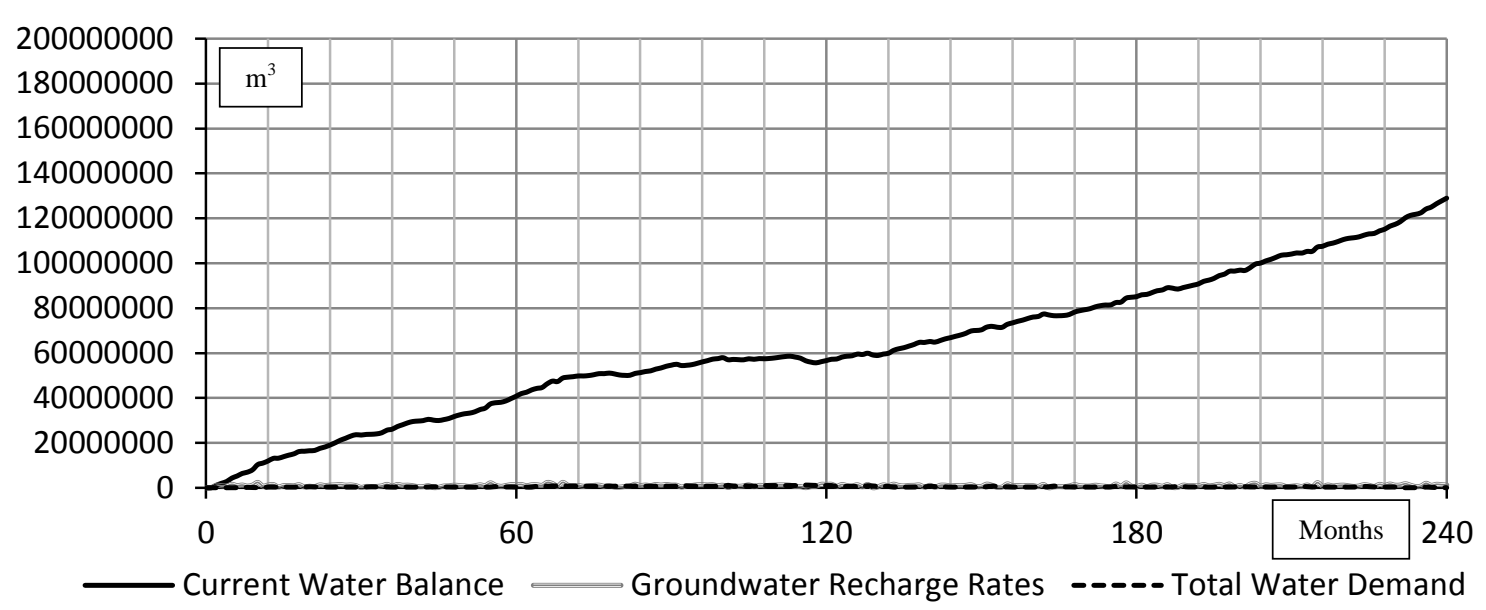

(a) Historic Climate Conditions

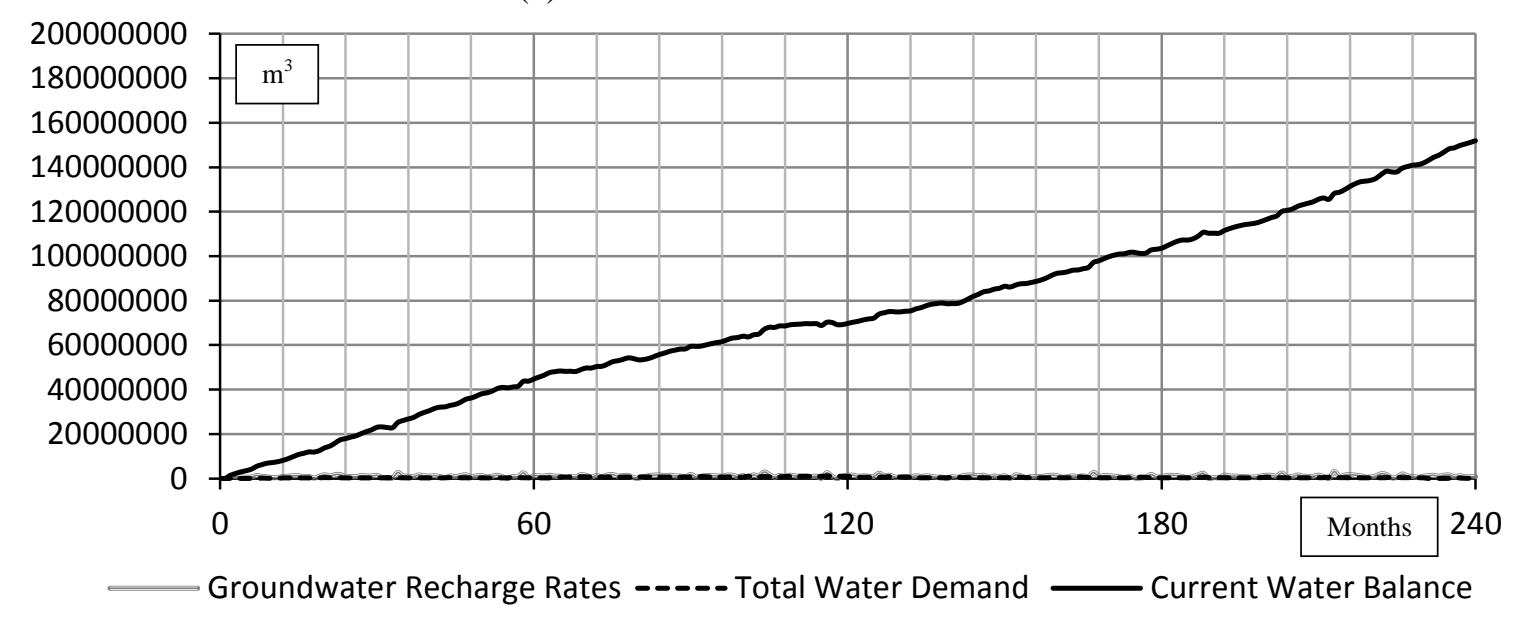

(b) Wet Climate Conditions

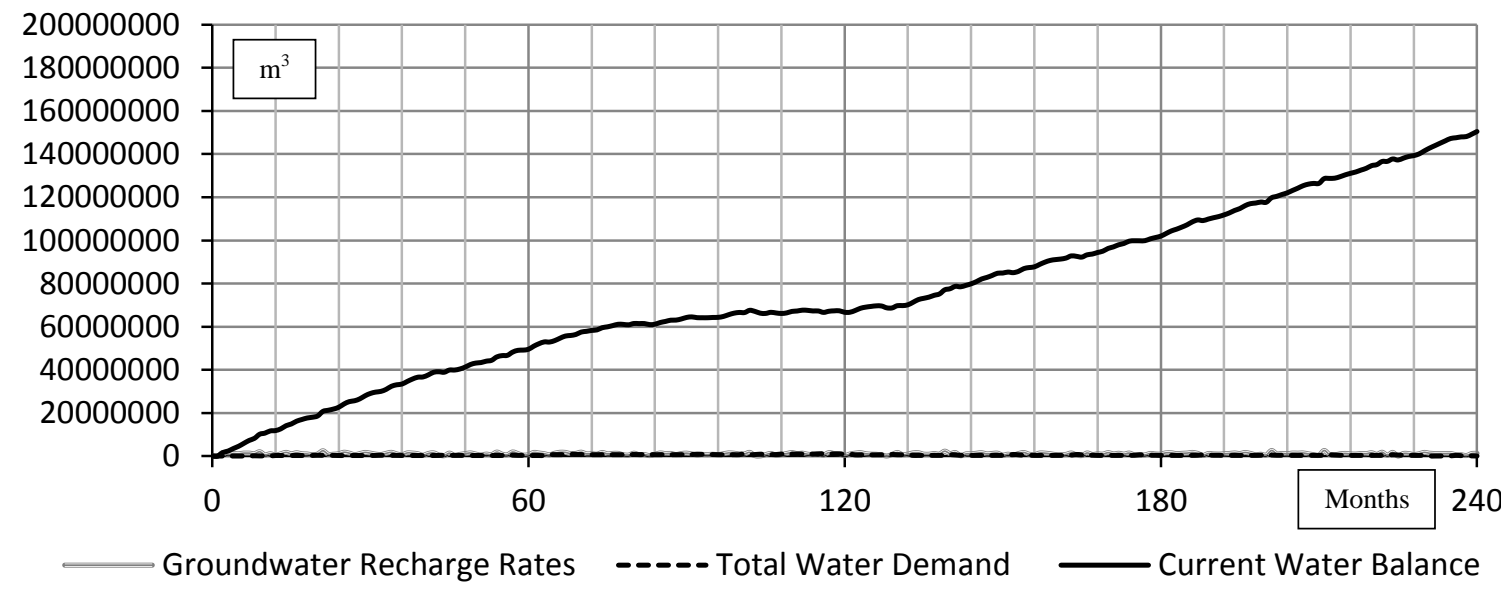

(c) Dry Climate Conditions

Figure 55: Middle Thames (sub-basin 14) cumulative water balance for three climate scenarios $(a, b, c)$ 


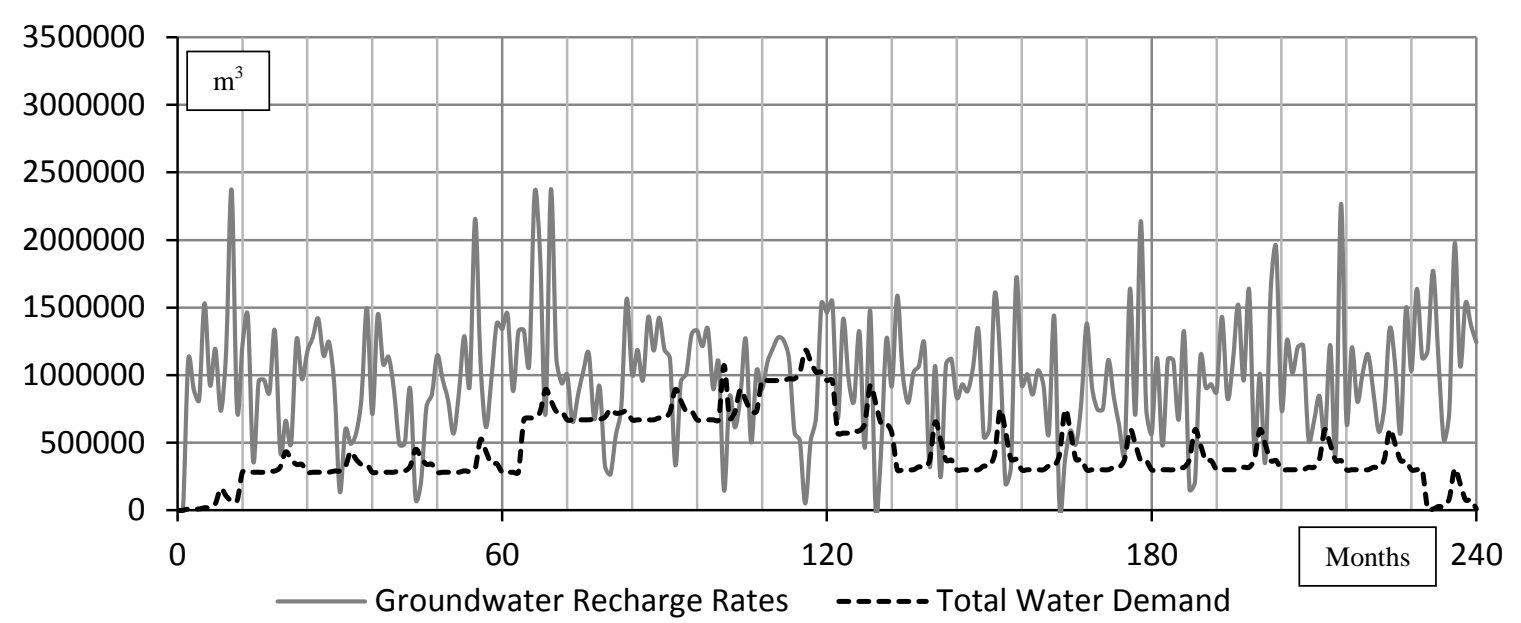

(a) Historic Climate Conditions

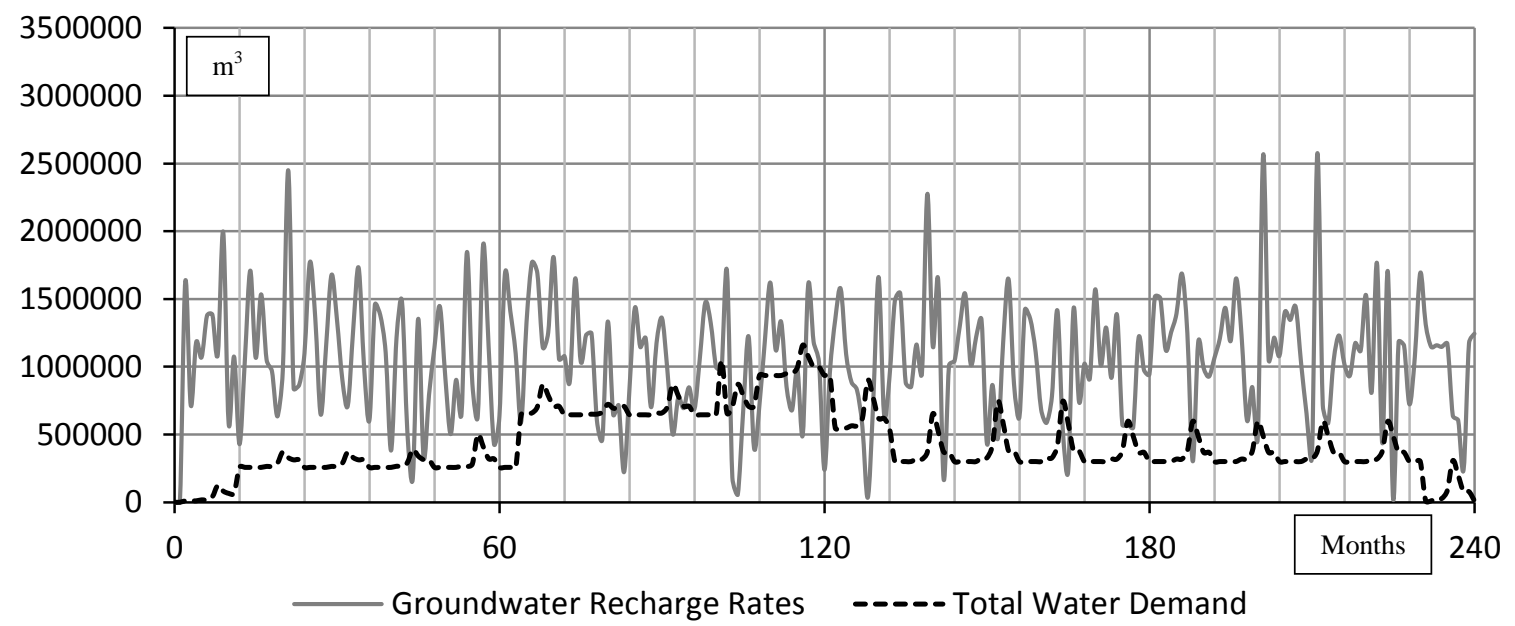

(b) Wet Climate Conditions

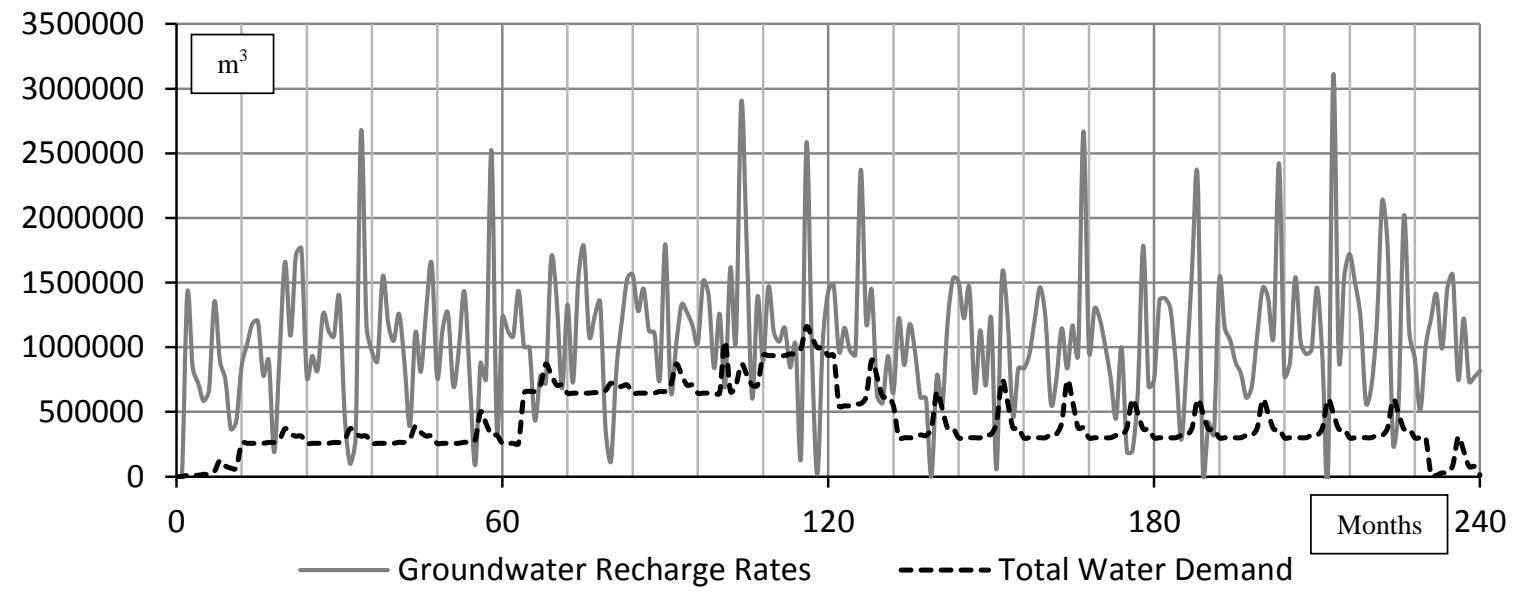

(c) Dry Climate Conditions

Figure 56: North Mitchell (sub-basin 20) groundwater recharge rates and total water demand for three climate scenarios $(a, b, c)$ 


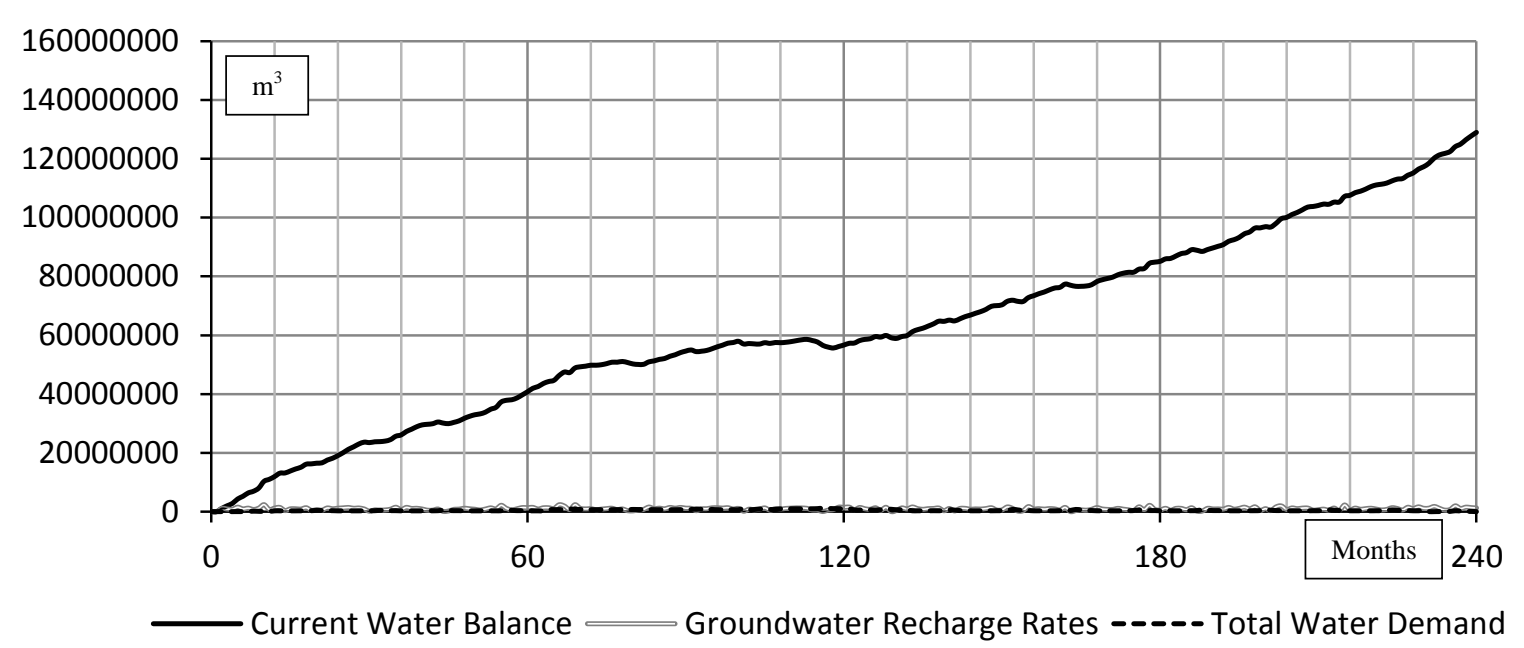

(a) Historic Climate Conditions

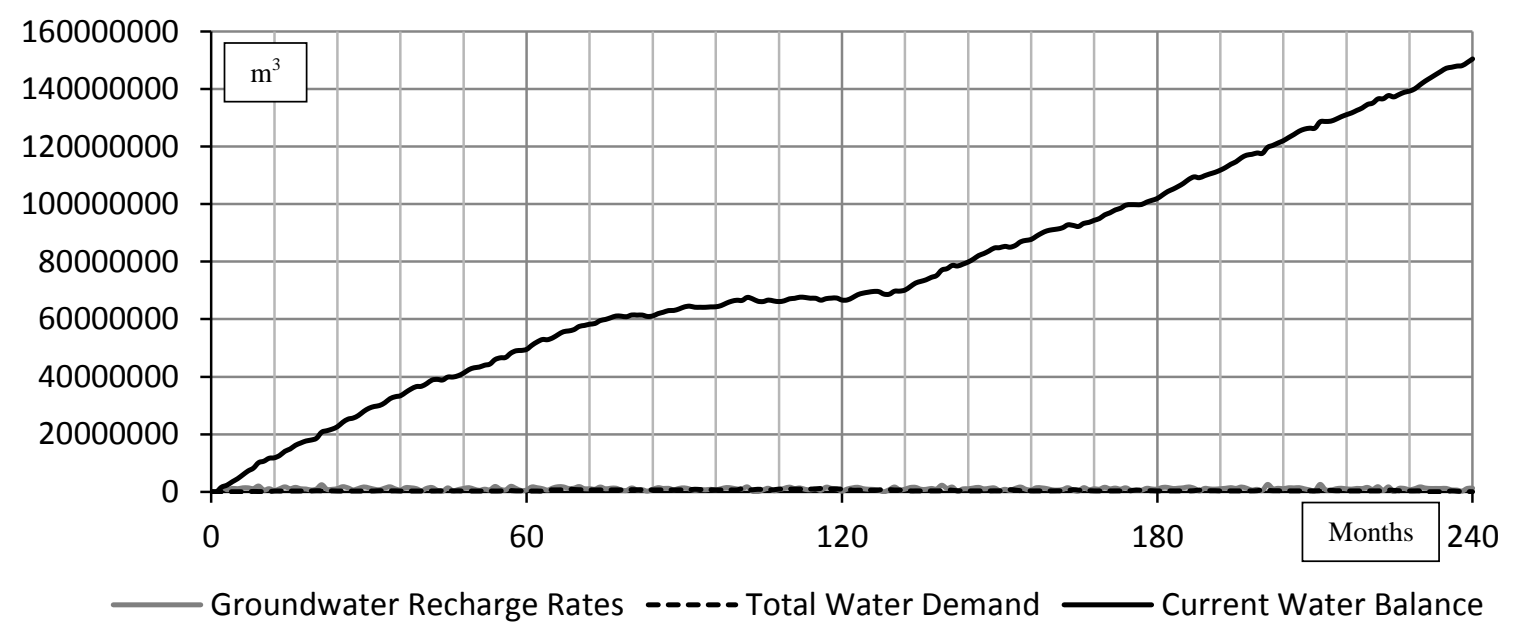

(b) Wet Climate Conditions

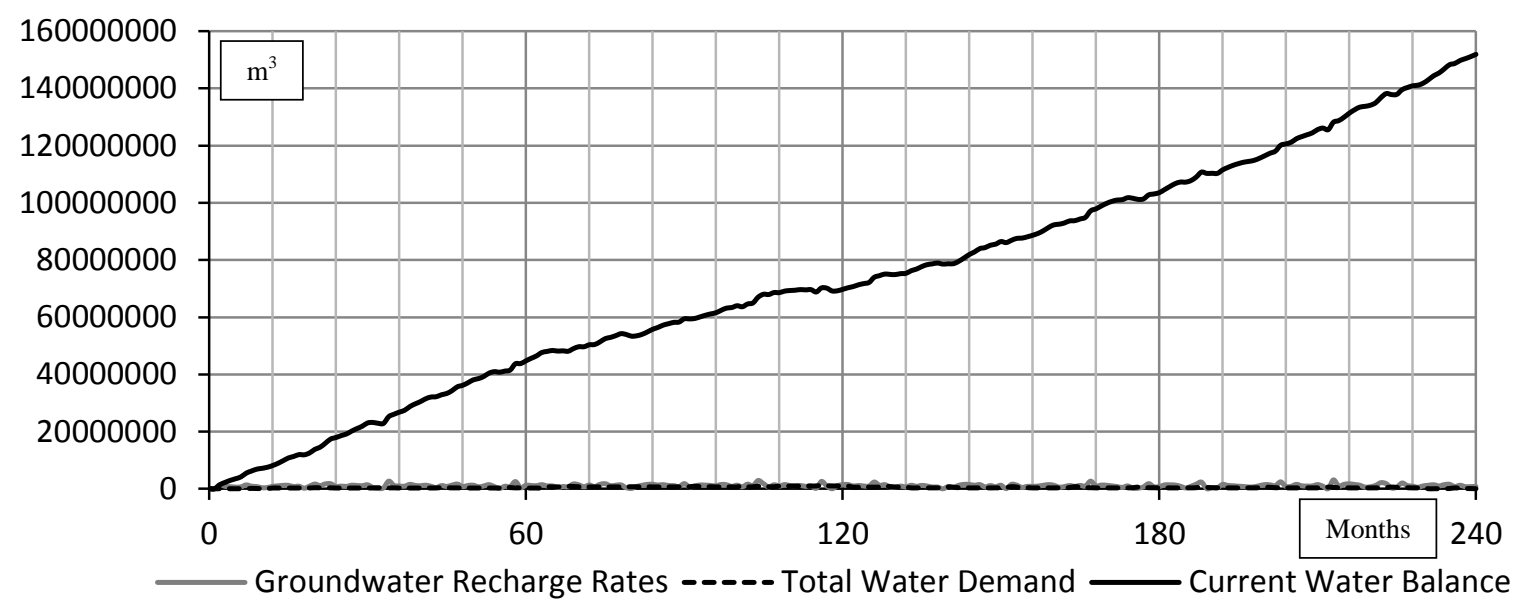

(c) Dry Climate Conditions

Figure 57: North Mitchell (sub-basin 20) cumulative water balance for three climate scenarios $(a, b, c)$ 


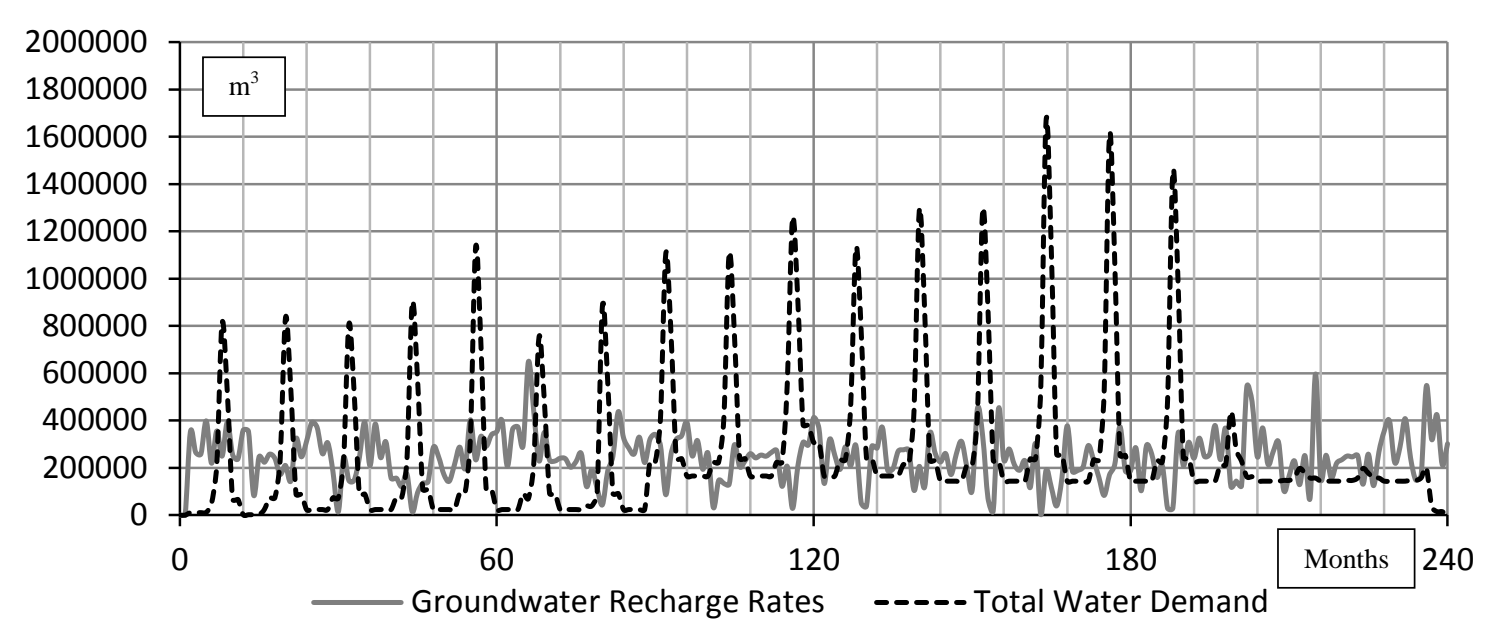

(a) Historic Climate Conditions

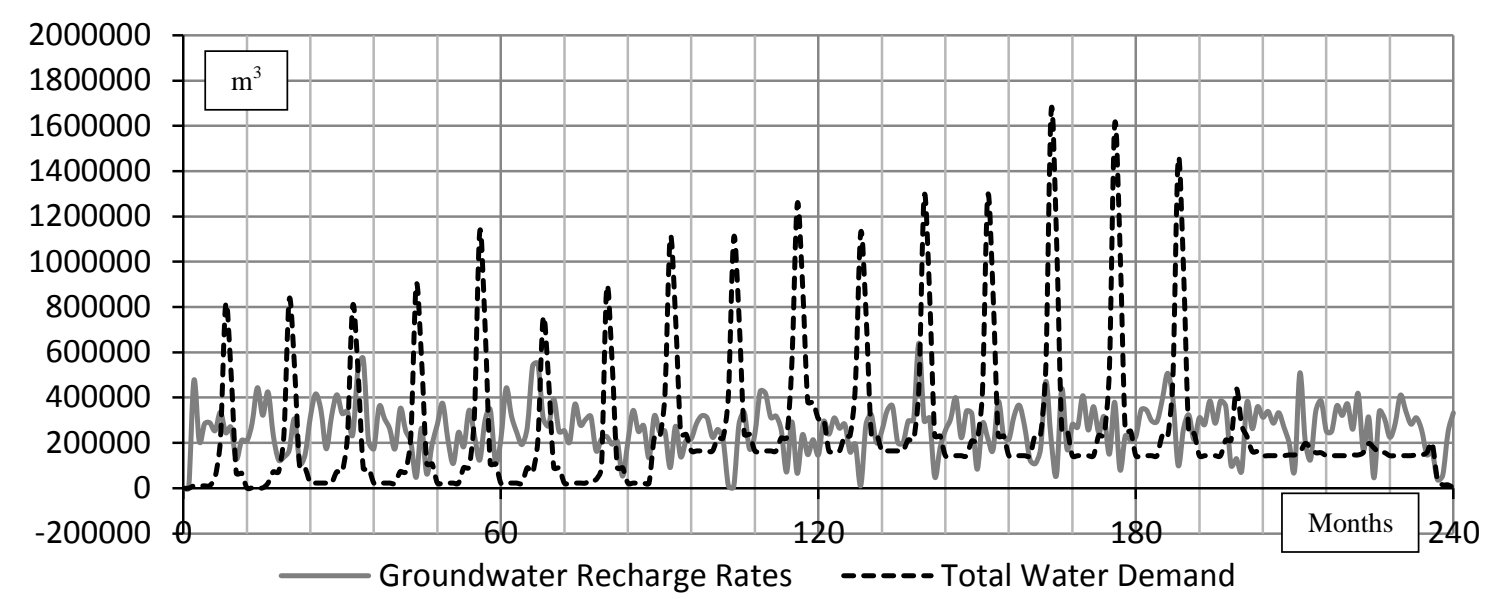

(b) Wet Climate Conditions

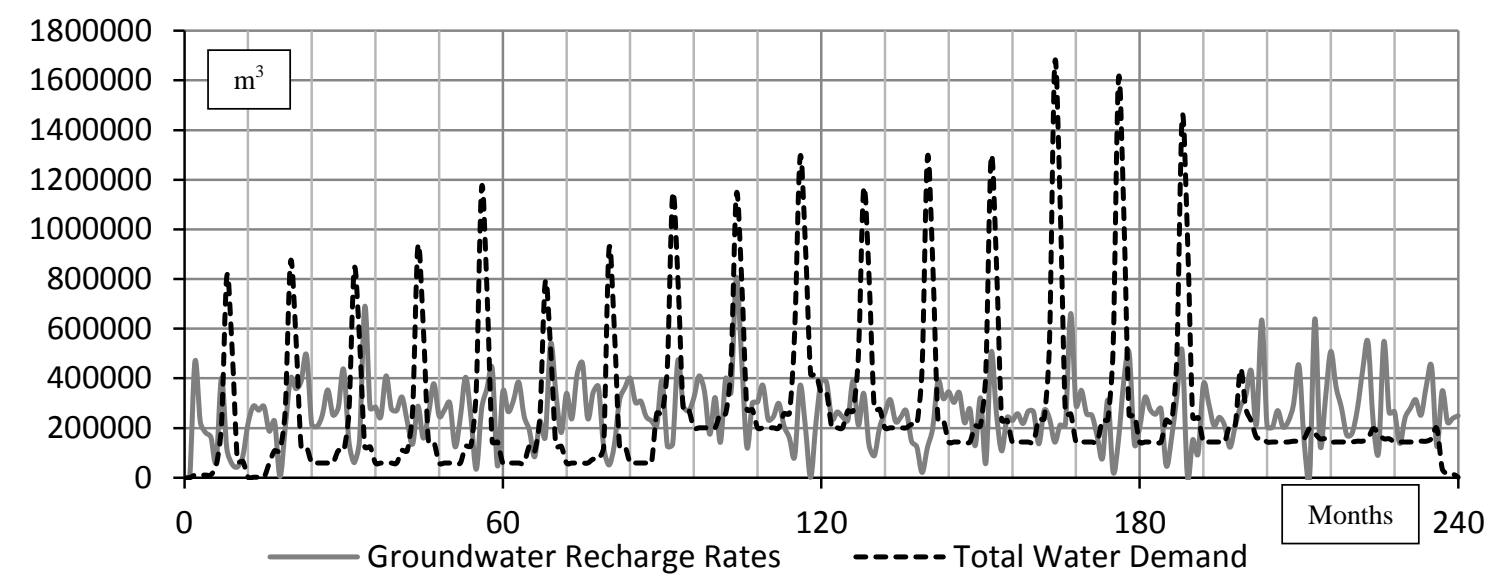

(c) Dry Climate Conditions

Figure 58: River Bend (sub-basin 27) groundwater recharge rates and total water demand for three climate scenarios $(a, b, c)$ 


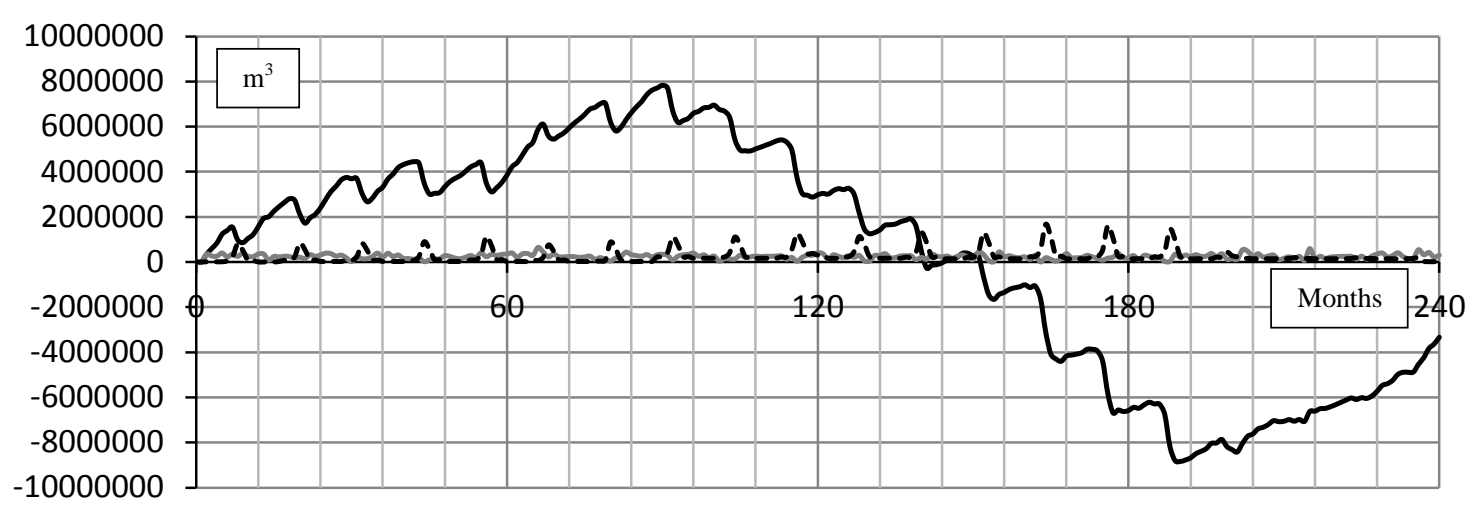

Current Water Balance Groundwater Recharge Rates ----- Total Water Demand

(a) Historic Climate Conditions

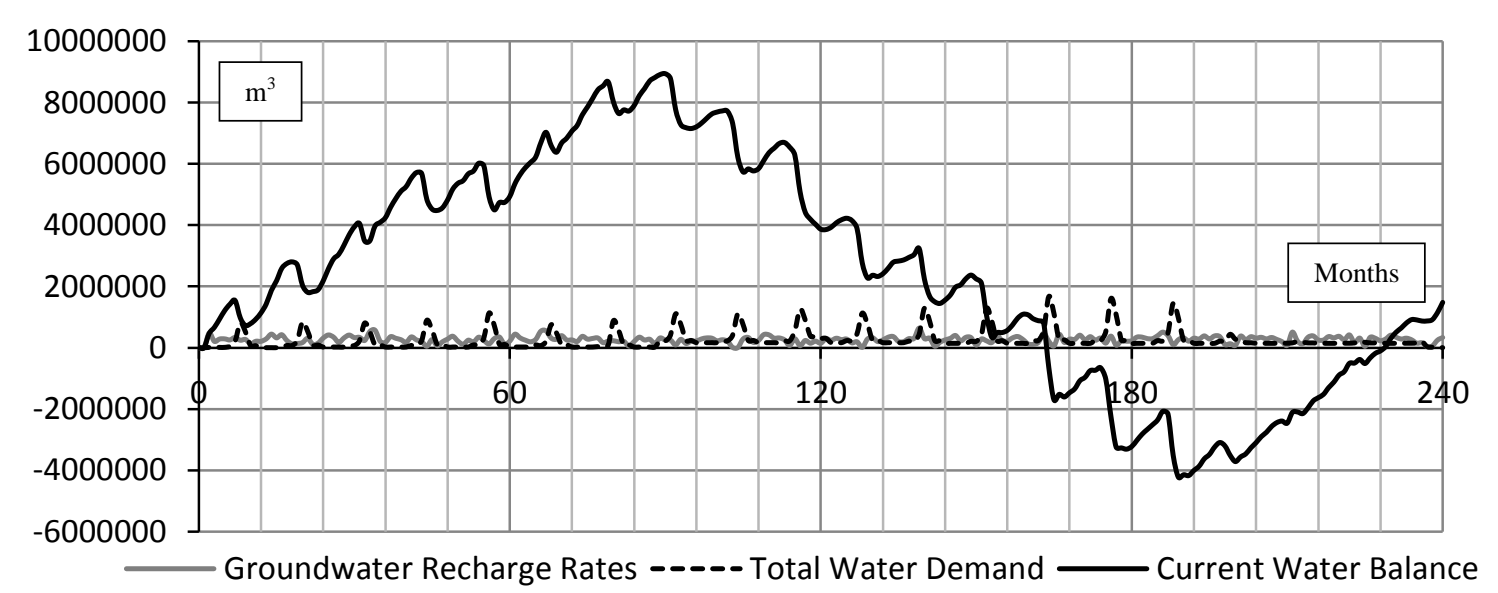

(b) Wet Climate Conditions

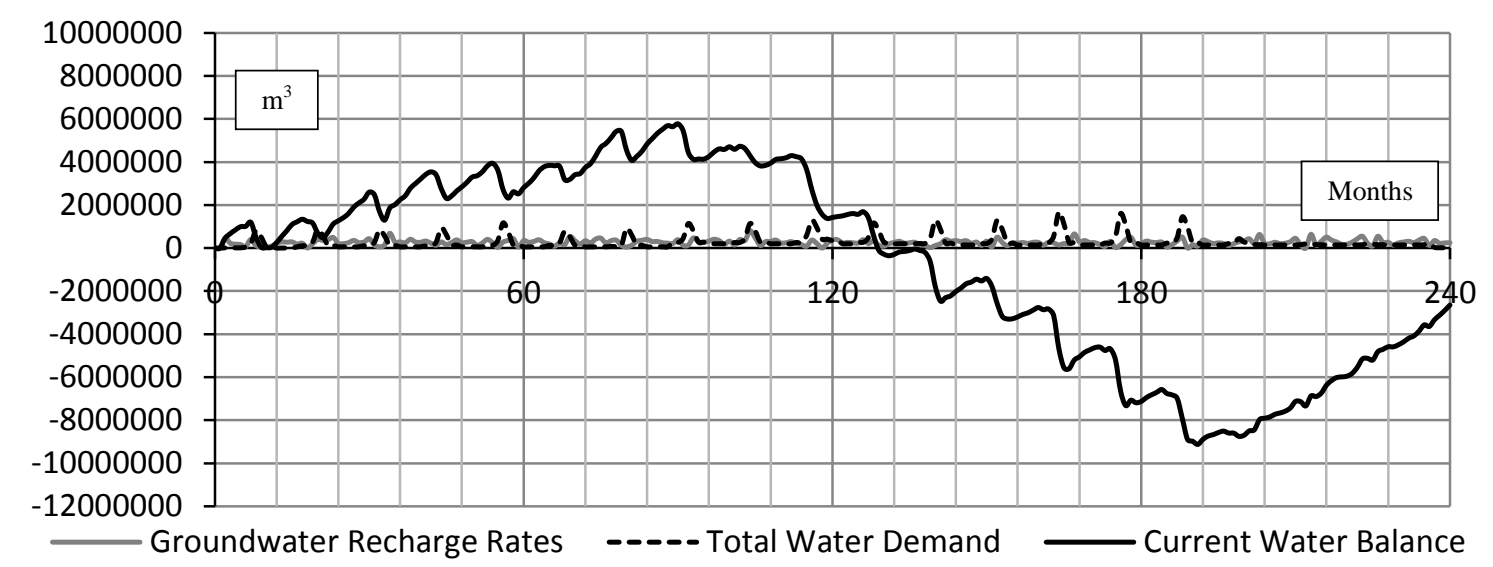

(c) Dry Climate Conditions

Figure 59: River Bend (sub-basin 27) cumulative water balance for three climate scenarios $(a, b, c)$ 


\subsection{Experiment 4: the infinite natural resources socio- economic scenario (ii) and historic climate conditions} (a)

In contrast to the baseline socio-economic scenario, this scenario examines the system behavior under the belief that the river basin contains inexhaustible natural and water resources. It therefore gives priority to all socio-economic processes disregarding the state of environment. Historic climate conditions use the unmodified historical records, and extended it to the period between January 1st, 2000 and January 1st, 2020.

Figures 60 to 64 illustrate the urbanization and land-use change (deforestation and reforestation) processes in five-year time steps $(1,61,121,181$, and 241). Grey patches represent urbanized areas at the start of simulation, January $1^{\text {st }}$, 2000, while white patches represent newly urbanized areas in the basin in each time step. In contrast to the baseline scenario, these figures demonstrate higher rates of urbanization. Also, they present slightly lower reforestation of the Upper Thames River basin. In addition, these figures show all active water users (agricultural, commercial, industrial and municipal) in the respective time steps. Figure 65 presents the actual rates of land-use change in $\mathrm{km}^{2}$ and demonstrates the rapid decline of agricultural lands and increase of residential areas and woodlands. Stronger economic growth in the region defined by this scenario is expected to create even more jobs and attract more people to the region. As a result, Figure 66 illustrates the steady dynamics of the population growth in the City of London. In order to promote a high standard of living, population density in the city decreases, resulting in a more progressive urbanization, Figure 67. On the other side, the sensitivity of local population to lack of water is higher due to high living standard expectations, but it doesn't affect the demographic growth due to the presence of adequate water resources.

Since water is not seen as a limiting factor, a significant socio-economic growth is observed as a result. Figure 68 shows that all individual water users have extended their permits to take water, allowing them to take more water for longer periods of time. Consequently, their individual demand is increased significantly, since their economic activity directly depends on the water quantities used, Figure 69. The levels of nonpermitted agricultural and domestic water consumption are also increased. As a result, 
compared to the base line socio-economic scenario, this scenario demonstrates significant increase in economic activity, Figure 70 - 72, despite the fact that the prices of products remain unchanged compared to the baseline scenario. Since the water resources are not seen as a limiting factor, the agricultural sector is also expected to thrive as a result of growing population and increased food demand. However, as more agricultural land is converted to housing land for individual homes, this is expected to create significant conflicts in the future. For that reason, the rates of reforestation are decreased.

However, such rates of economic and social growth are expected to have even greater consequences on the regional hydrology. The high rates of urbanization are expected to influence the groundwater recharge rates since a larger portion of rainfall ends up in rivers and streams, thus increasing the volumes of surface runoff. Figure 73(a) shows the resulting River Thames median monthly flow rates at Byron station for historic climate conditions. The median flow rate for the period of simulation is $26.52 \mathrm{~m}^{3} / \mathrm{s}$, while the average flow is $31.45 \mathrm{~m}^{3} / \mathrm{s}$. Figure 74 (a) presents the median monthly flow rates at Ingersoll. The median flow rate for the period of twenty years is $9.95 \mathrm{~m}^{3} / \mathrm{s}$, while the average flow is $12.05 \mathrm{~m}^{3} / \mathrm{s}$. Finally, Figure 75 (a) shows the same results at St.Marys where the median flow rate is $3.62 \mathrm{~m}^{3} / \mathrm{s}$, and the average is $4.24 \mathrm{~m}^{3} / \mathrm{s}$. These values are higher than the one obtained for the combination of baseline socio-economic and historic climate conditions. Comparative analysis of resulting runoffs is given in Chapter 5.8.

As a result of urbanization, less water is infiltrated to the groundwater aquifers that many municipalities use for their drinking water supply. At the higher spatial scales, such as the level of three counties (Middlesex, Oxford and Perth), the differences between natural recharge rates and water demand only seasonal and they allow for sustainable use of groundwater aquifers in current climate conditions, Figures 76(a), 77(a) and 78(a). This suggests that groundwater aquifers in these three counties are not in danger of overexploitation. However, as in the base line scenario, the situation changes on the lower sub-basin scales (Middle Thames, North Mitchell, and River Bend). Figures 79(a) and 81(a) compare the balance between monthly groundwater recharge rates and the total demand for two sub-basins - Middle Thames and North Mitchell. Both figures show significantly higher water demand rates in the second half of simulation. The cumulative 
water balance presented in Figures 80(a) and 82(a) decays in the second period of simulation, and shows that a significantly higher water demand puts pressure on both regions. On the other hand, compared to the baseline scenario, River Bend sub-basin experiences even more severe shortages of groundwater recharge rates due to the number of water users located in the sub-basin. Figure 83(a) describes the obvious difference between groundwater recharge rates and water demand and shows that local groundwater aquifers do not receive enough water to naturally recharge, not even in current climate conditions. The cumulative water balance presented in Figure 84(a) shows that, in the long term, the groundwater recharge rates are not sufficient to satisfy the increased demand. However, just like in the base line scenario case, finite conclusions should be followed by detailed studies of groundwater movements and interactions between local aquifers and the Great Lakes.

\subsection{Experiment 5: the infinite natural resources socio- economic scenario (ii) and wet climate conditions (b)}

This scenario analyzes the system behavior under the extreme socio-economic and wet climate conditions. The historical data are modified by the wet climate scenario to represent a climate that is wetter and warmer than normal. Since the model parameters used to describe the infinite natural resources socio-economic scenario remain unchanged, the socio-economic results are presented in Figures 60 to 72. However, compared to the historic climate conditions, the main difference brought by the wet scenario refers to changes in hydrologic regime. Figure 73(b) shows the obtained River Thames median monthly flow rates at Byron station for wet climate conditions. The median flow rate for the period of simulation is $29.45 \mathrm{~m}^{3} / \mathrm{s}$, while the average flow is $35.02 \mathrm{~m}^{3} / \mathrm{s}$. Figure 74 (b) presents the median monthly flow rates at Ingersoll. The median flow rate for the 20 years period is $10.88 \mathrm{~m}^{3} / \mathrm{s}$, while the average flow is $14.11 \mathrm{~m}^{3} / \mathrm{s}$. Finally, Figure 75 (b) shows the same results at St.Marys where the median flow rate is $3.95 \mathrm{~m}^{3} / \mathrm{s}$, and the average is $4.94 \mathrm{~m}^{3} / \mathrm{s}$. Figures $76(\mathrm{~b}), 77(\mathrm{~b})$ and $78(\mathrm{~b})$ present the seasonal variations of ground water recharge rates and total water demand for three counties (Middlesex, Oxford and Perth) in the Upper Thames River basin for the wet climate scenario. Results show only seasonal imbalance between natural recharge and demand 
rates. This suggests that groundwater aquifers at the level of these three counties are not in danger of overexploitation under the wet climate conditions, in spite of significantly increased water demand. Similarly to the historic climate condition, the situation changes at the lower spatial scales (the sub-basin level). Figure 79(b) and Figure 81(b) compare the monthly rates of groundwater recharge to monthly total water demand for described socio-economic conditions for wet climate scenario for two sub-basins, Middle Thames and North Mitchell. Figures 80(b) and 82(b) present the cumulative water balance accounted for both sub-basins, Middle Thames and North Mitchell, and demonstrate a sign of overutilization. The observed trends suggest that, even in the case of wetter climate conditions but stronger socio-economic activity, the two sub-basins are must be carefully managed to avoid unsustainable use of groundwater resources. Compared to the historic climate scenario, the situation at River Bend sub-basin is just slightly healthier. Figure 83(b) shows monthly variation in groundwater demand and groundwater recharge rates, and illustrates still present pressure on local water resources, even in the wet climate conditions. Long-term cumulative water balance, presented in Figure 84(b), demonstrates that the local groundwater recharge rates are still not sufficient to sustainably replenish groundwater aquifers due to extensive water withdrawals.

\subsection{Experiment 6: the infinite natural resources socio- economic scenario (ii) and dry climate conditions (c)}

This experiment couples the infinite natural resources socio-economic and dry climate conditions. The dry climate scenario describes a drier and cooler climate, which might lead to dry, spells and droughts. The model parameters, shown in Table 29, used to define the baseline socio-economic scenario remain unmodified, and the socio-economic results are presented in Figures 60 to 72. In this drier climate, the hydrologic regime indicates some change. Figure 73(c) shows the obtained River Thames median monthly flow rates at Byron station for dry climate conditions. The median flow rate for the period of 20 years is $26.35 \mathrm{~m}^{3} / \mathrm{s}$, while the average flow is $28.51 \mathrm{~m}^{3} / \mathrm{s}$. Figure $74(\mathrm{c})$ presents the median monthly flow rates at Ingersoll. The median flow rate for the period of simulation is $9.83 \mathrm{~m}^{3} / \mathrm{s}$, while the average flow is $11.32 \mathrm{~m}^{3} / \mathrm{s}$. Finally, Figure $75(\mathrm{c})$ shows the same results at St.Marys where the median flow rate is $3.41 \mathrm{~m}^{3} / \mathrm{s}$, and the average is $3.94 \mathrm{~m}^{3} / \mathrm{s}$. 
Figures 76(c), 77(c) and 78(c) show the seasonal variations of ground water recharge rates and total water demand for three counties for the dry climate scenario. The results at the county level illustrate more frequent imbalance between natural recharge and demand rates. However, groundwater aquifers in these three counties in long-terms are not in risk of overexploitation even in dry climate conditions. But the situation significantly differs at the sub-basin level spatial scales.

Figure 79(c) and Figure 81(c) present the monthly rates of groundwater recharge and monthly total water demand for described socio-economic conditions and the dry climate scenario for two sub-basins, Middle Thames and North Mitchell. These figures show only the seasonally higher water demand in the second part of the simulation. Figures 80(c) and 82(c) present the cumulative water balance accounted for both sub-basins, Middle Thames and North Mitchell, and, in long terms, show signs of overutilization. The observed trends suggest that, in the case of drier climate conditions, the two sub-basins should be carefully managed to support the future economic growth. On the other hand, the situation at River Bend sub-basin is even more alarming. Figure 83(c) shows monthly variation in groundwater demand and groundwater recharge rates, and reveals very significant seasonal pressures on local water resources. Long-term cumulative water balance, presented in Figure 84(c), demonstrates that the local groundwater recharge rates are insufficient to sustainably support additional socio-economic development. 


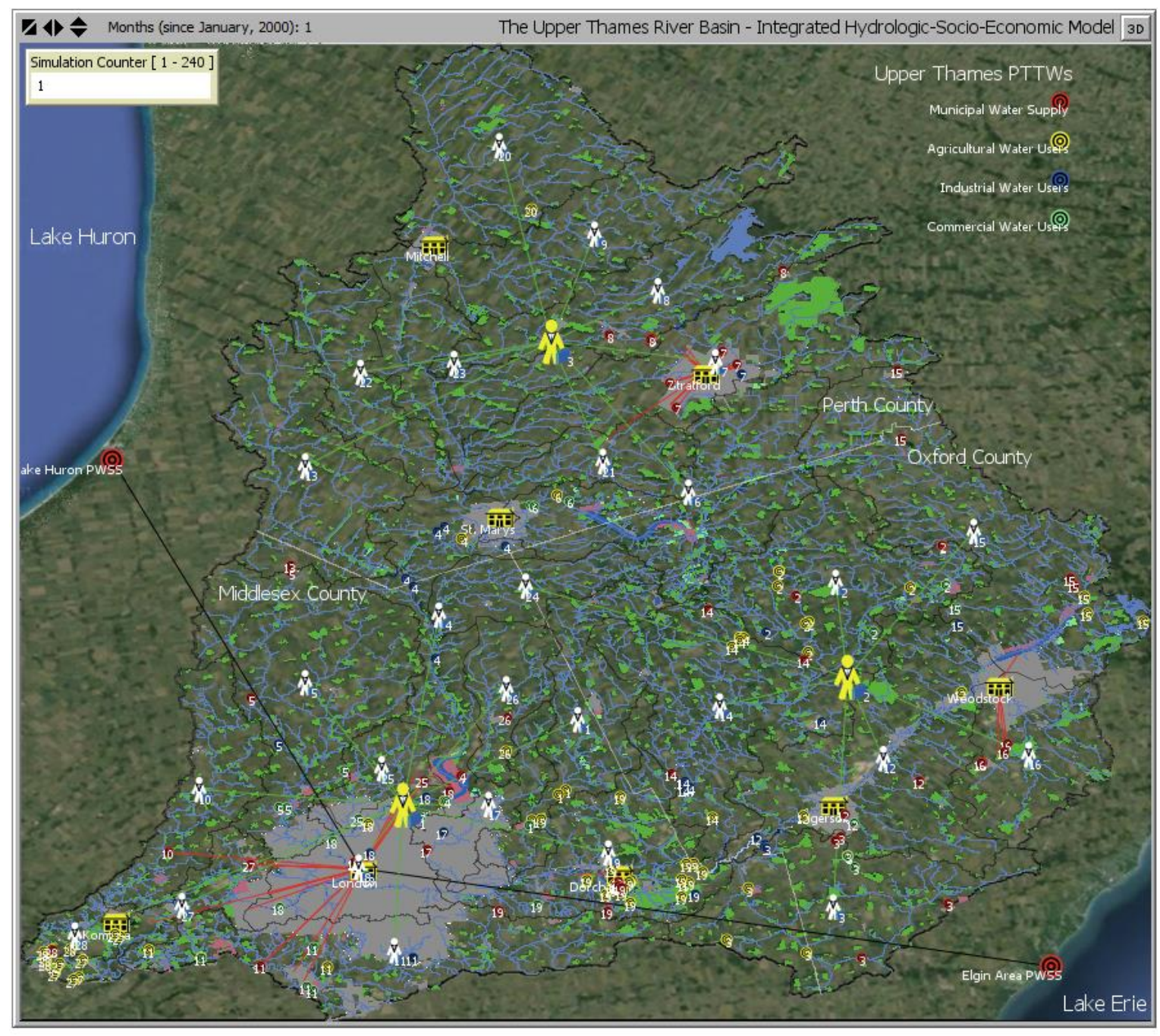

Figure 60: Dynamics of land use change and active water users in the Upper Thames

River basin at time step 1 (January, 2000) 


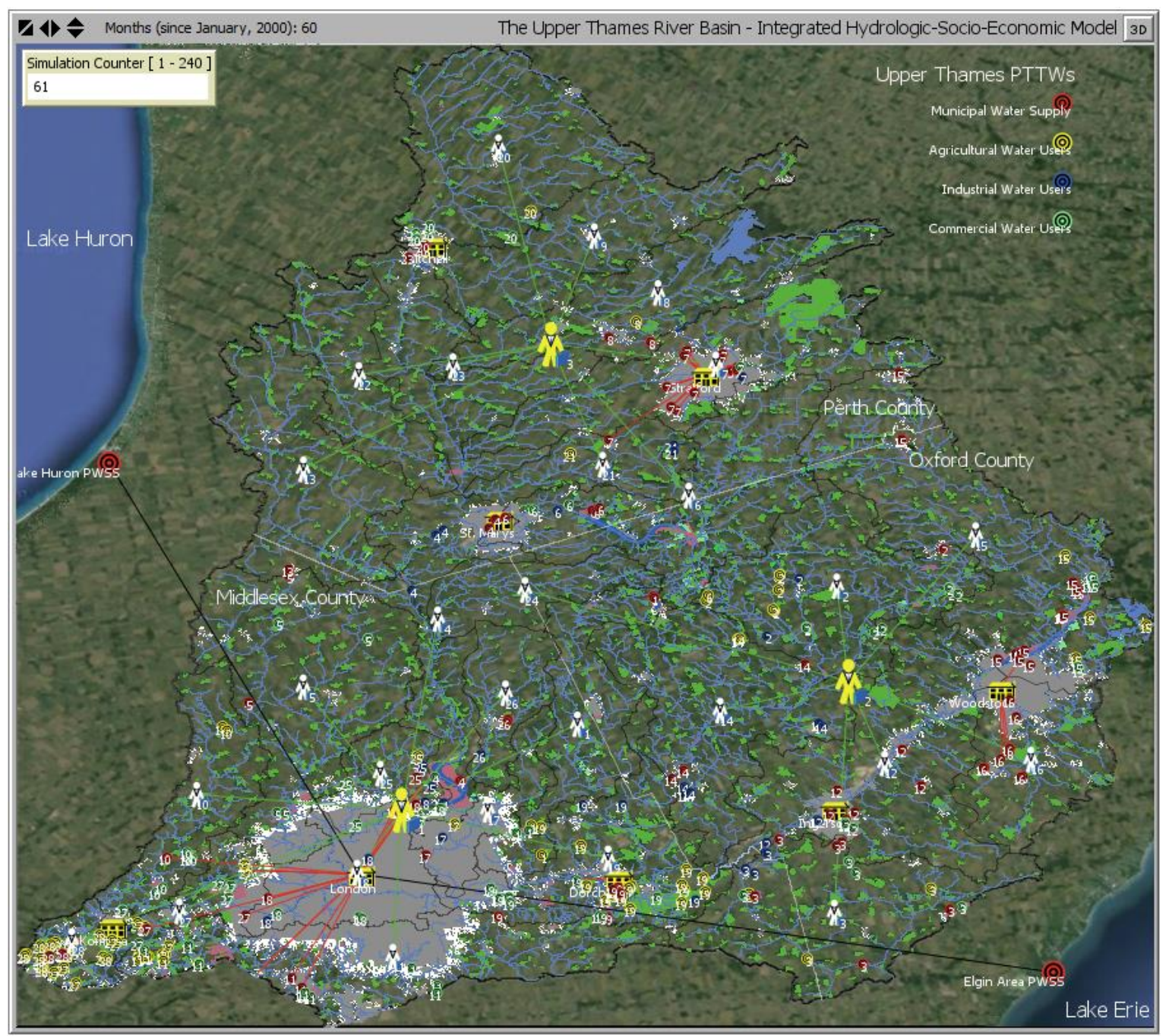

Figure 61: Dynamics of land use change and active water users in the Upper Thames River basin at time step 61 (January, 2005) 


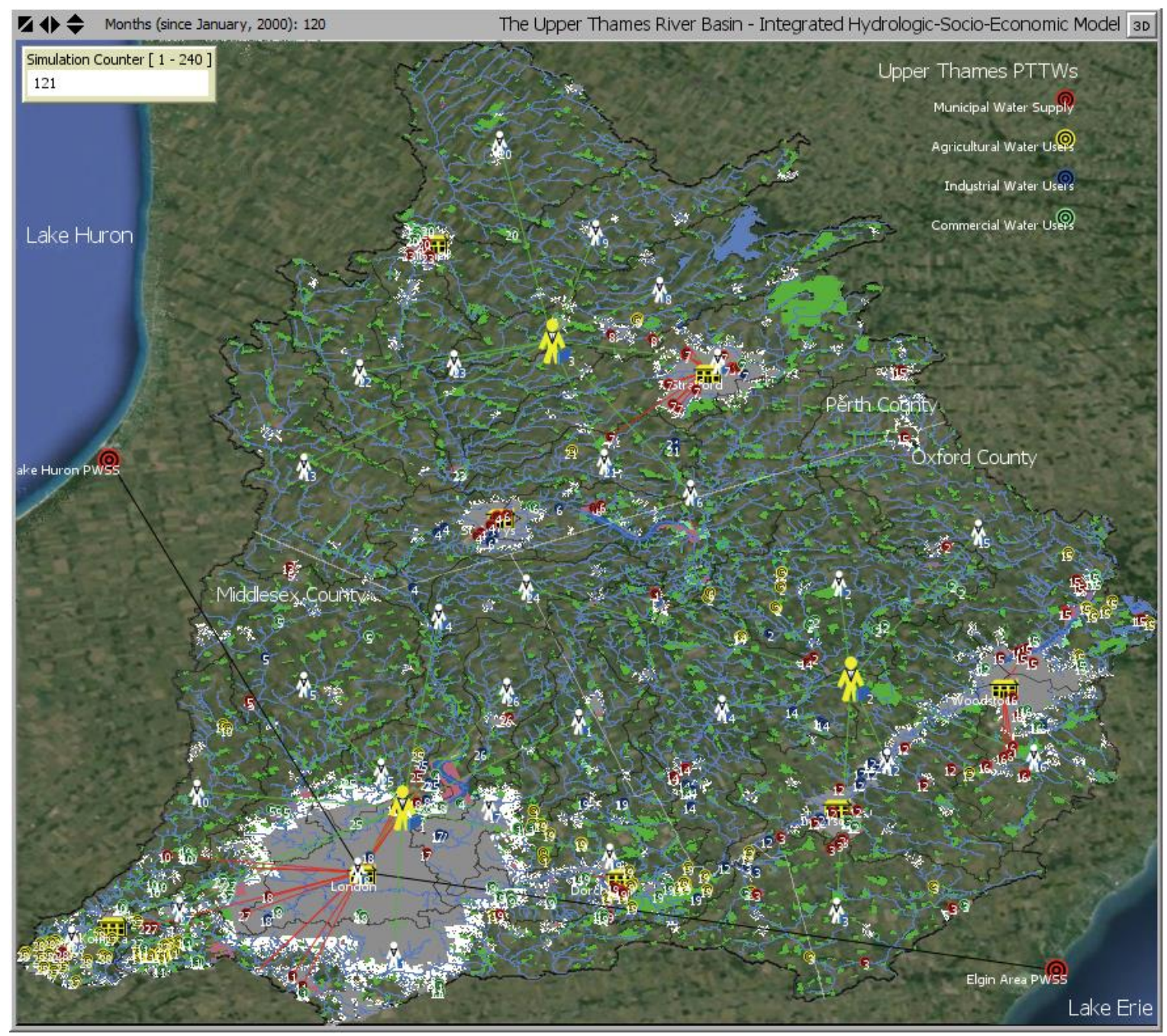

Figure 62: Dynamics of land use change and active water users in the Upper Thames

River basin at time step 121 (January, 2010) 


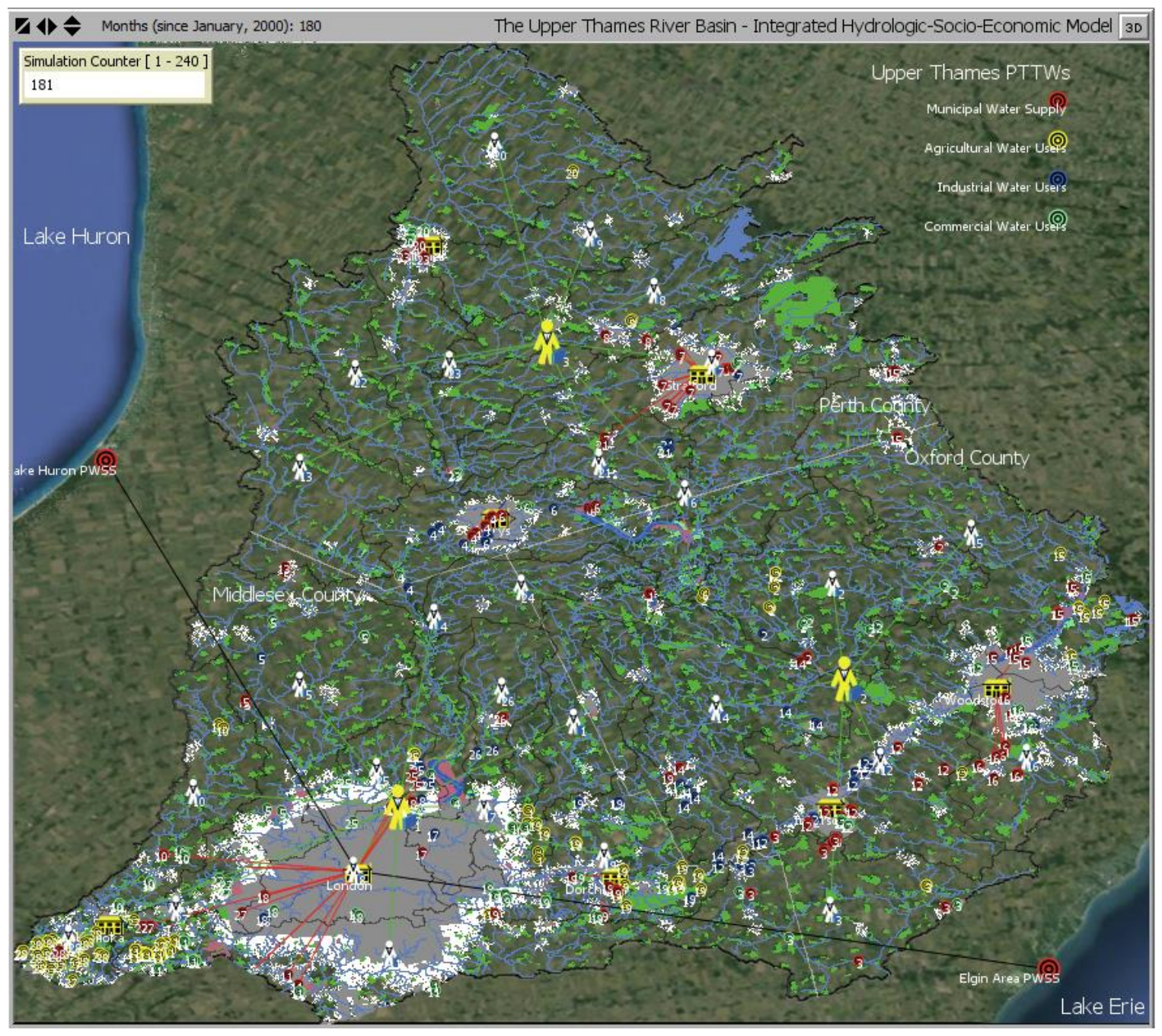

Figure 63: Dynamics of land use change and active water users in the Upper Thames River basin at time step 181 (January, 2015) 


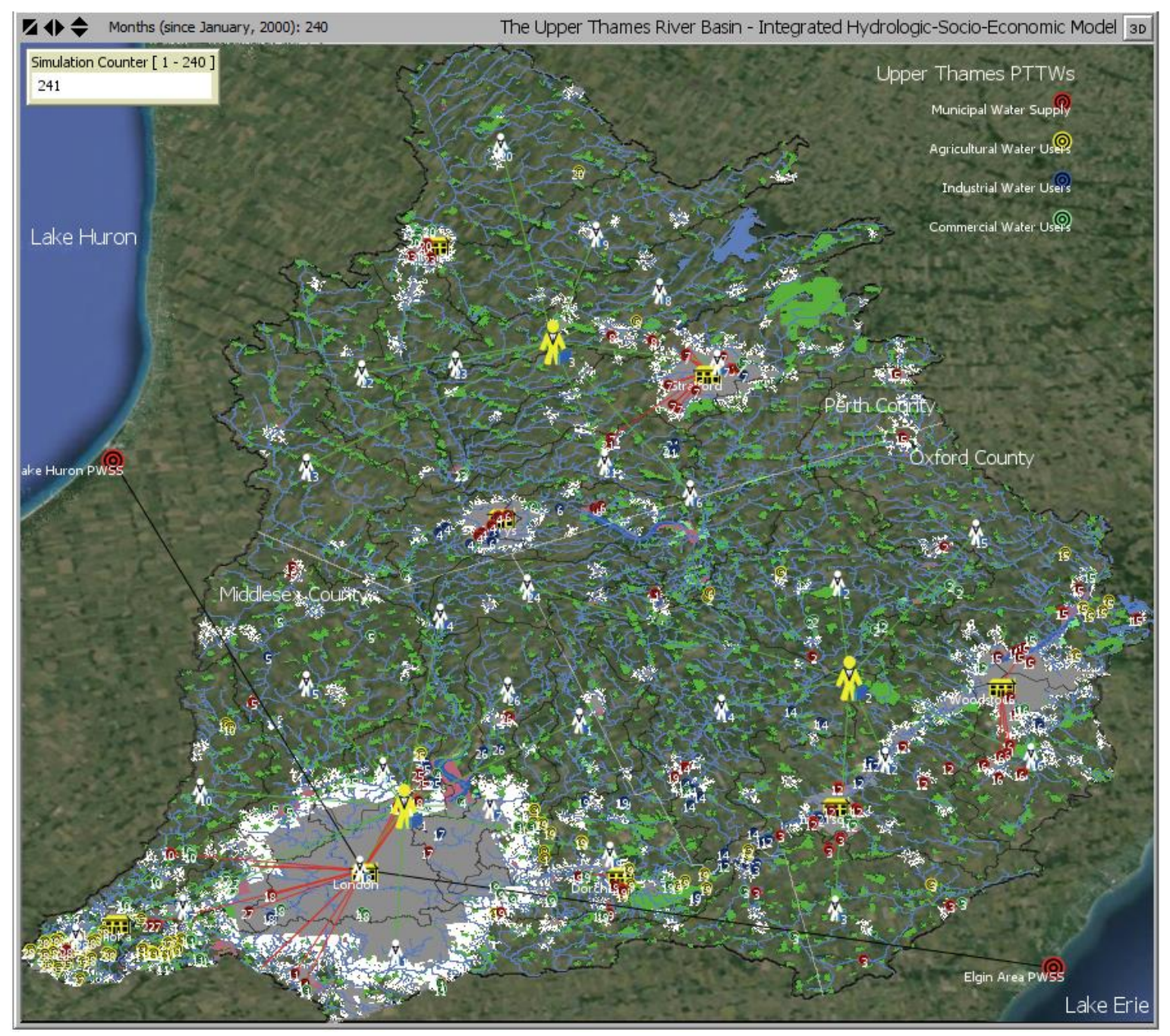

Figure 64: Dynamics of land use change and active water users in the Upper Thames River basin at time step 241 (January, 2020) 


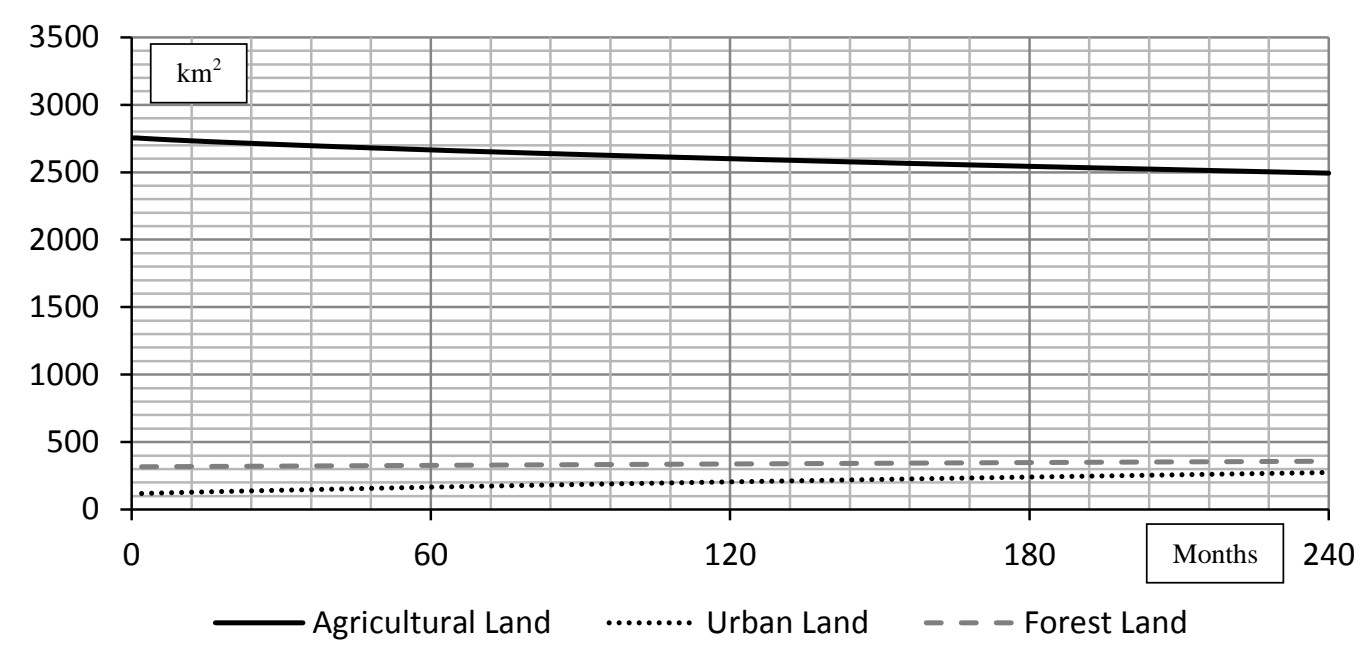

Figure 65: Types of land use categories in the UTRB

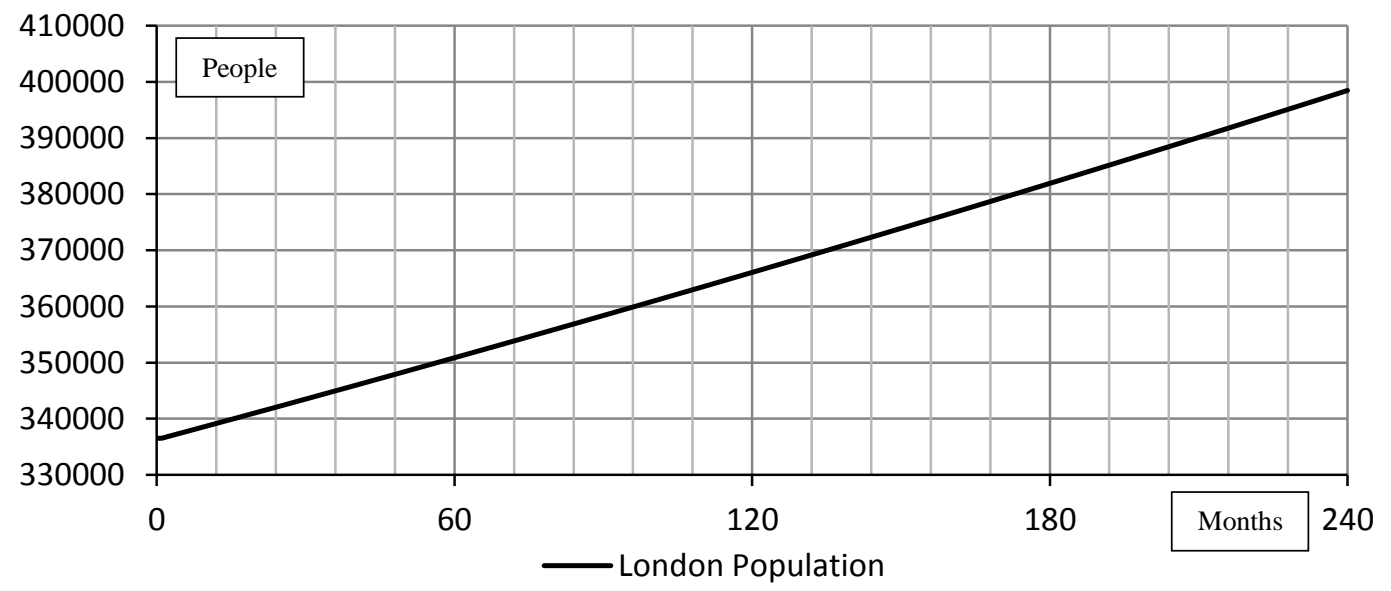

Figure 66: Population dynamics for the City of London

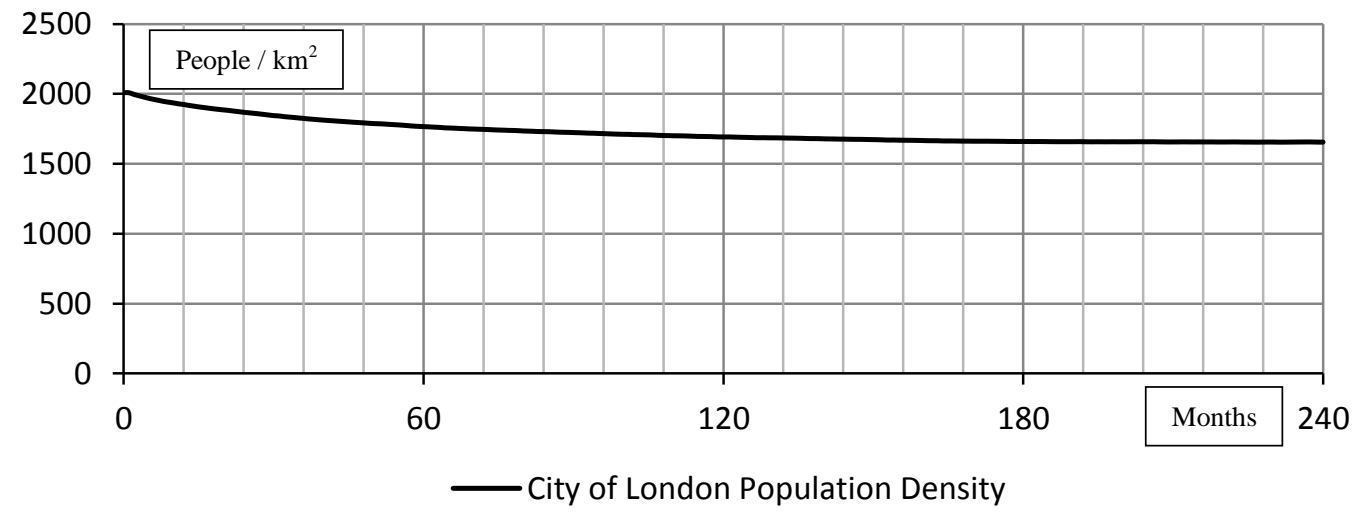

Figure 67: Population density for the City of London 


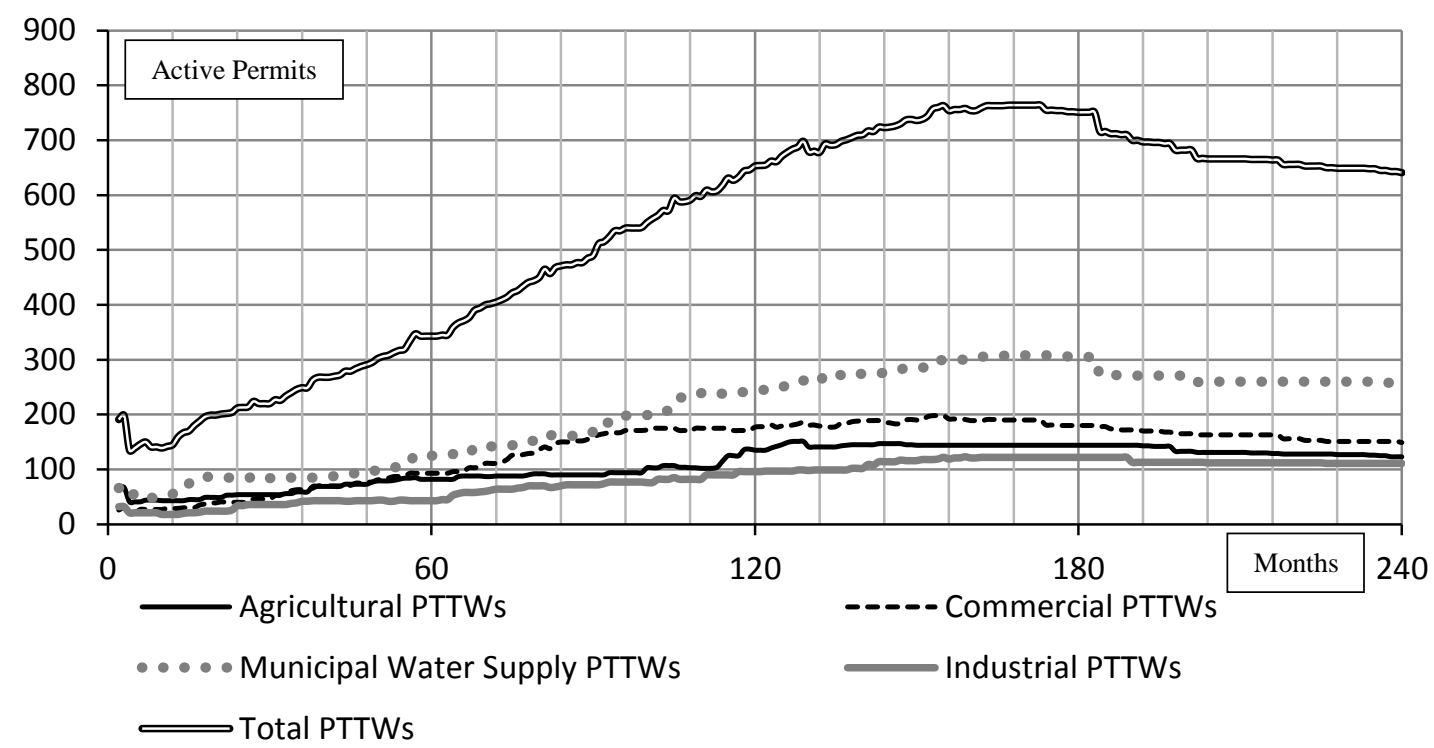

Figure 68: Active Permits to Take Water (PTTW)

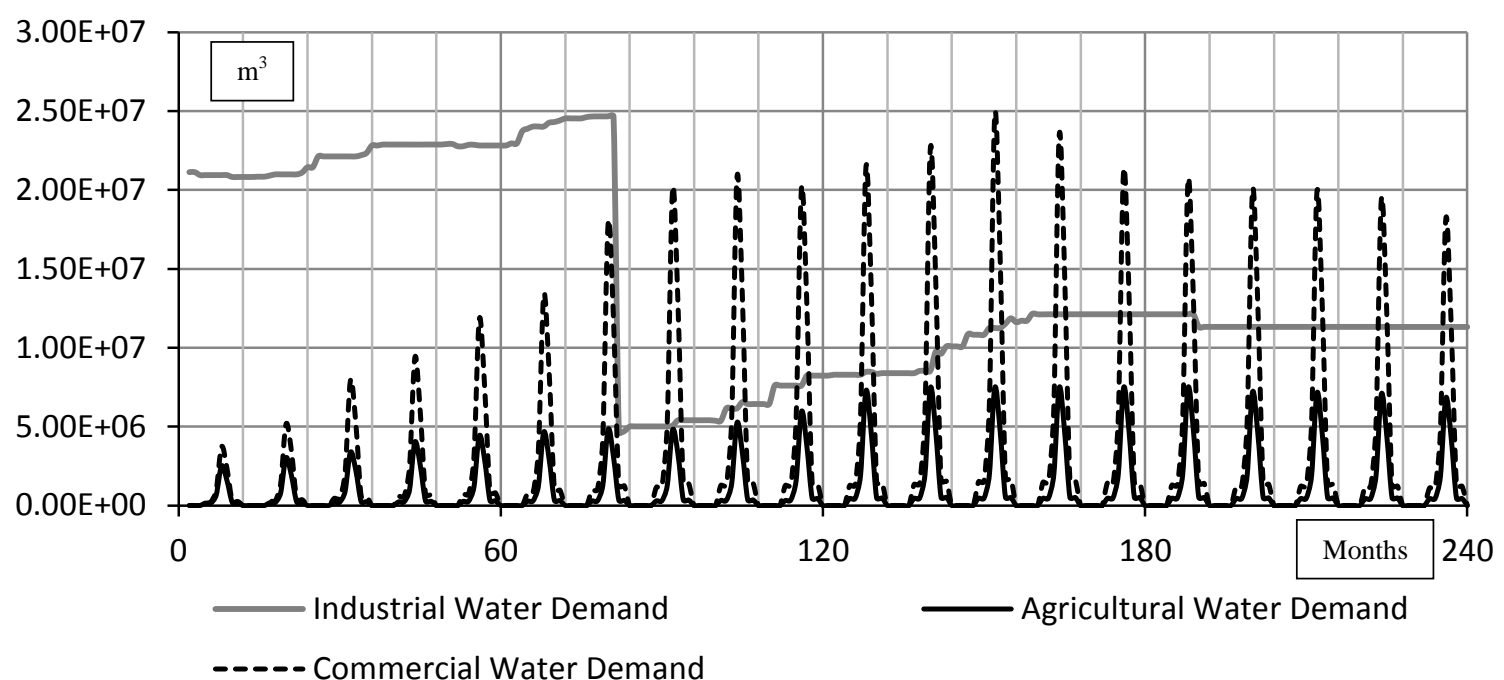

Figure 69: Monthly water demand by sector 


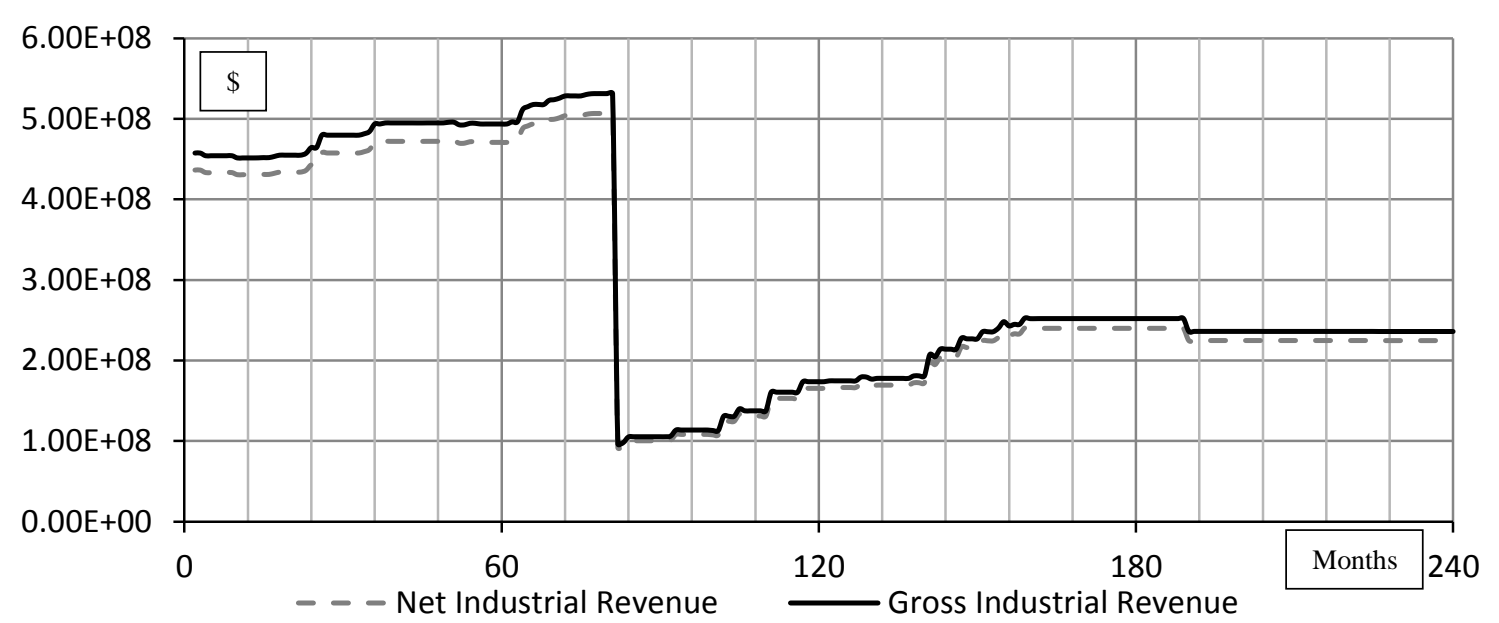

Figure 70: Industrial economic revenue based on the individual water demand

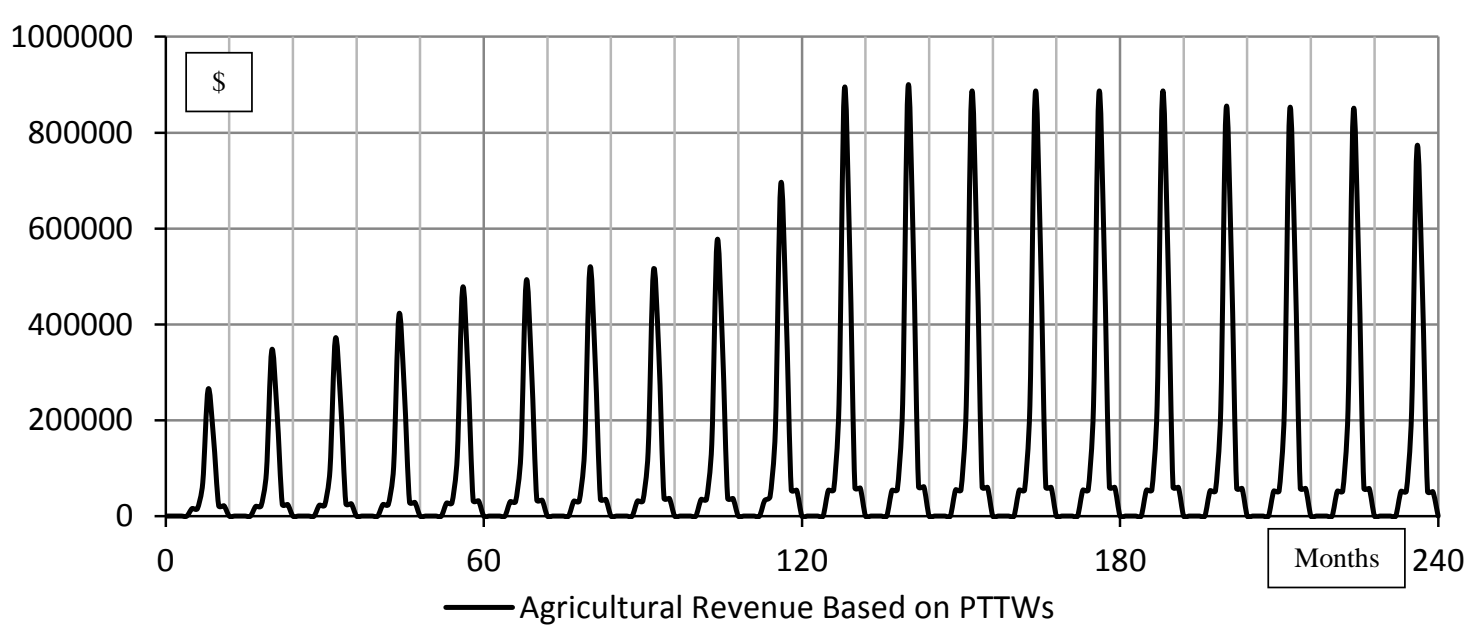

Figure 71: Economic revenues for agricultural water users based on the PTTW database

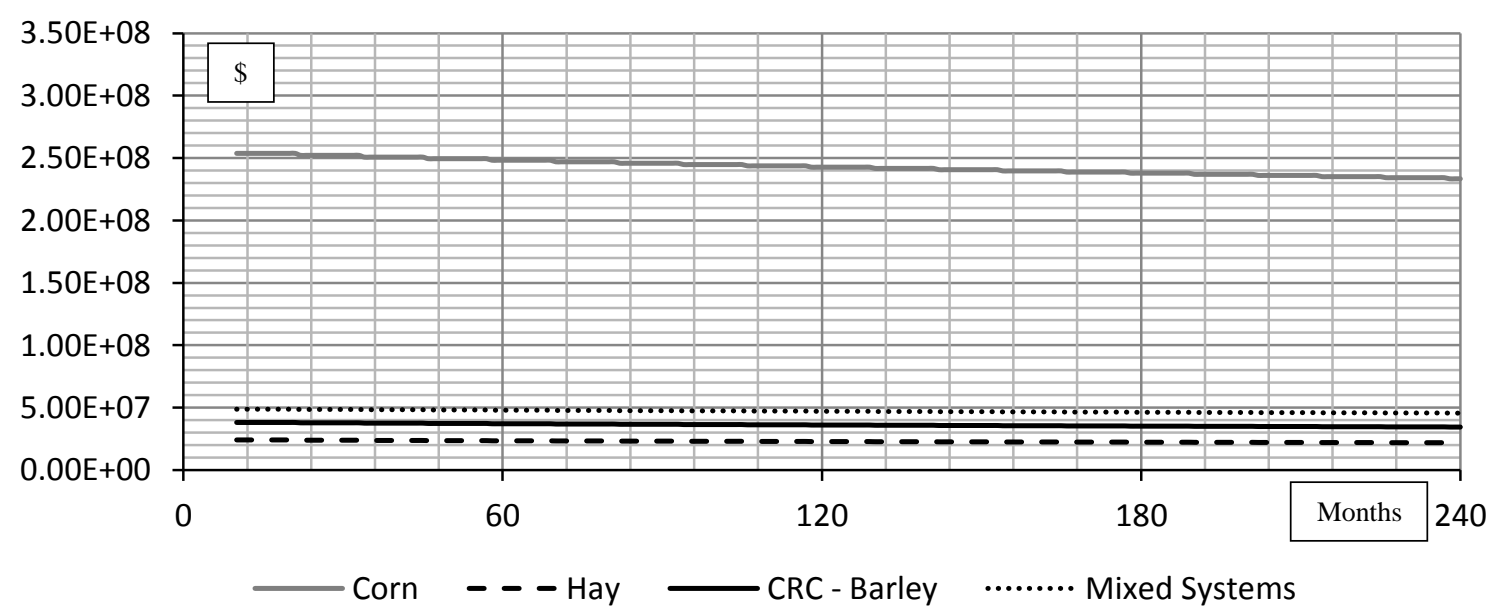

Figure 72: Agricultural economic revenue based on the farmed area 


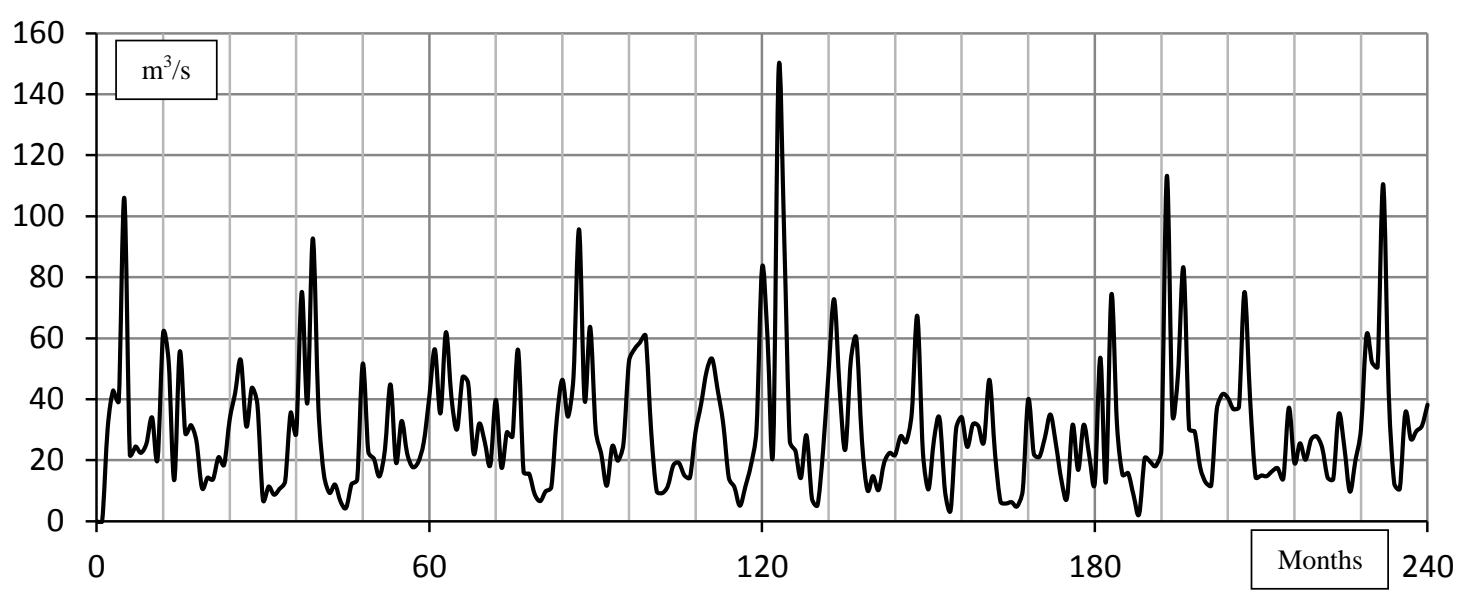

(a) Historic Climate Conditions

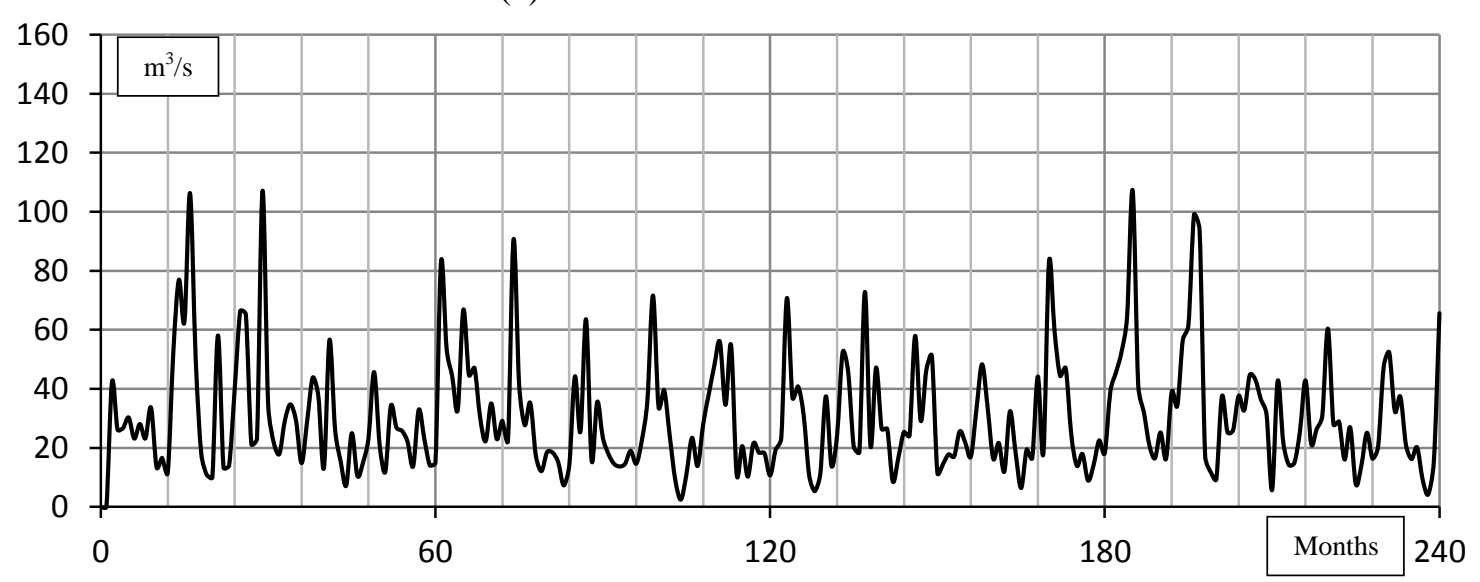

(b) Wet Climate Conditions

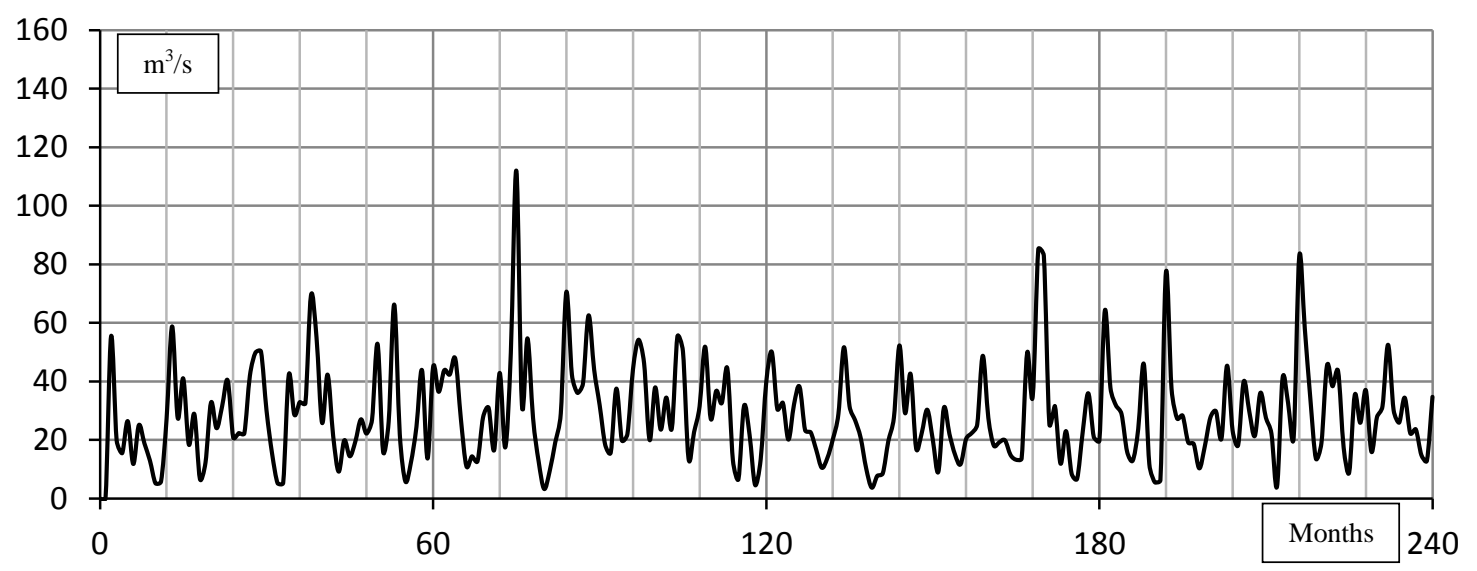

(c) Dry Climate Conditions

Figure 73: River Thames median monthly flow rates at Byron for three climate scenarios $(\mathrm{a}, \mathrm{b}, \mathrm{c})$ 


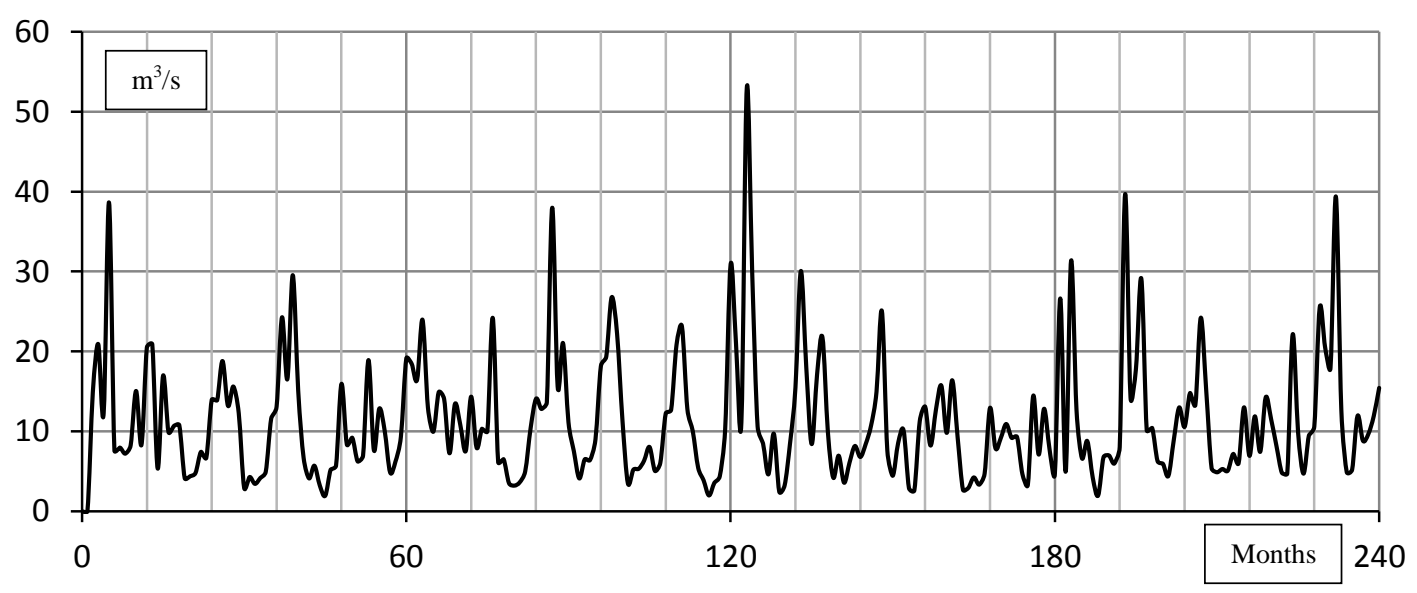

(a) Historic Climate Conditions

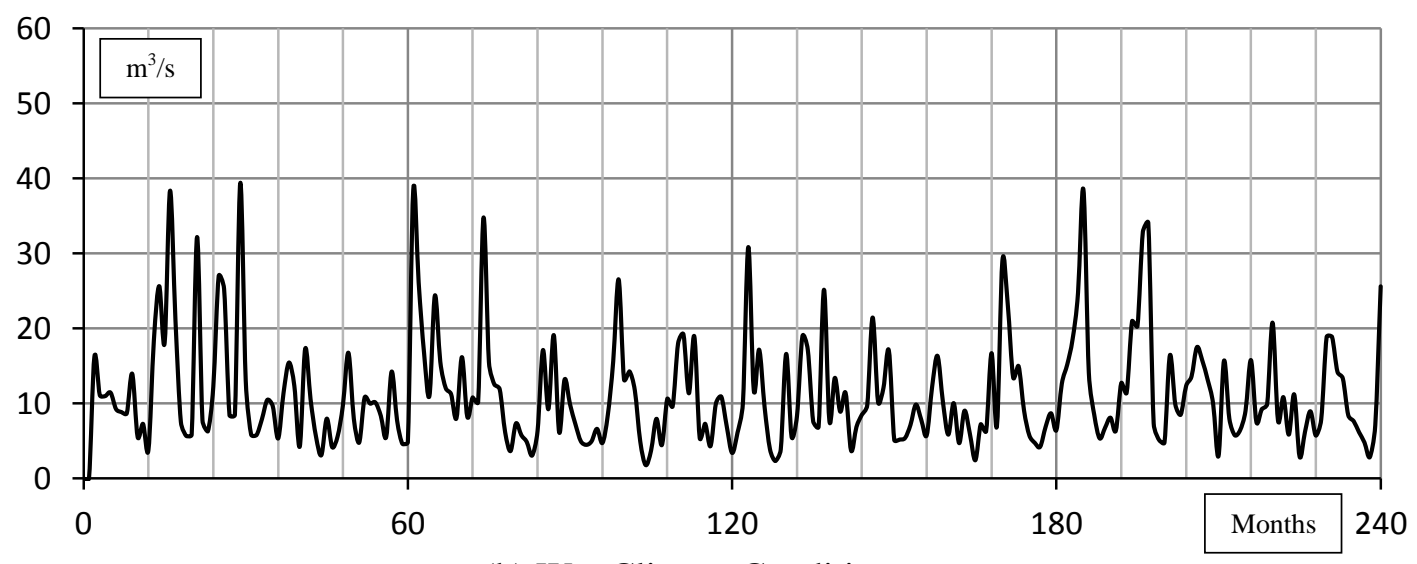

(b) Wet Climate Conditions

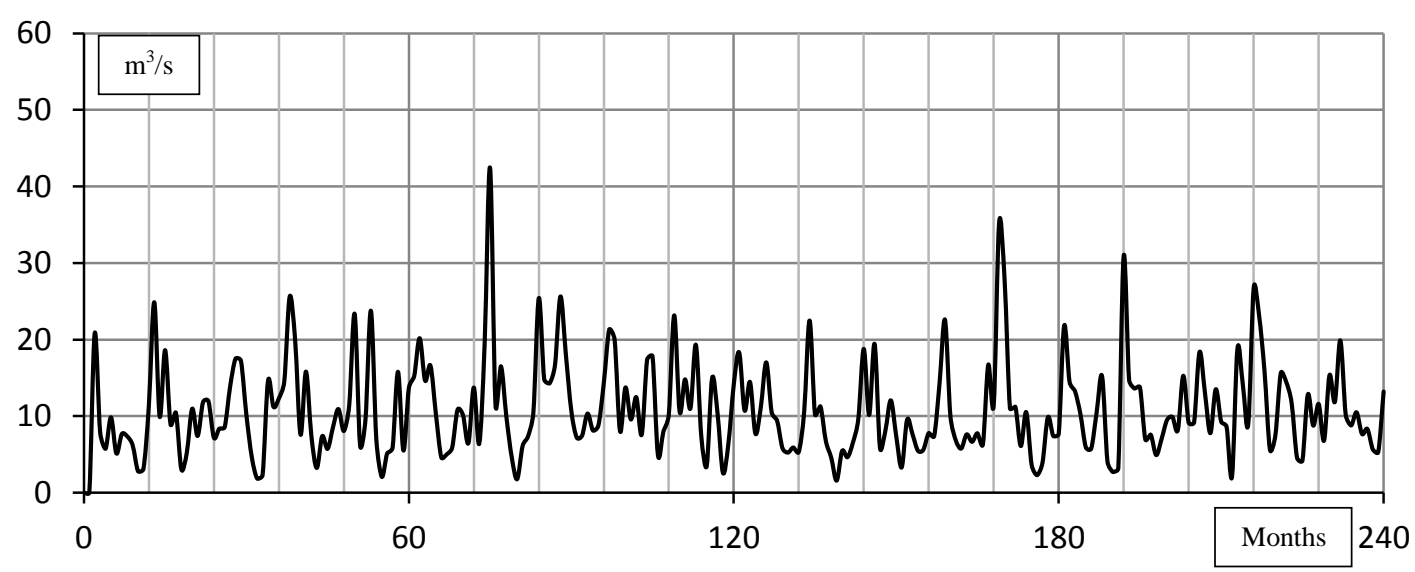

(c) Dry Climate Conditions

Figure 74: River Thames median monthly flow rates at Ingersoll for three climate scenarios $(a, b, c)$ 


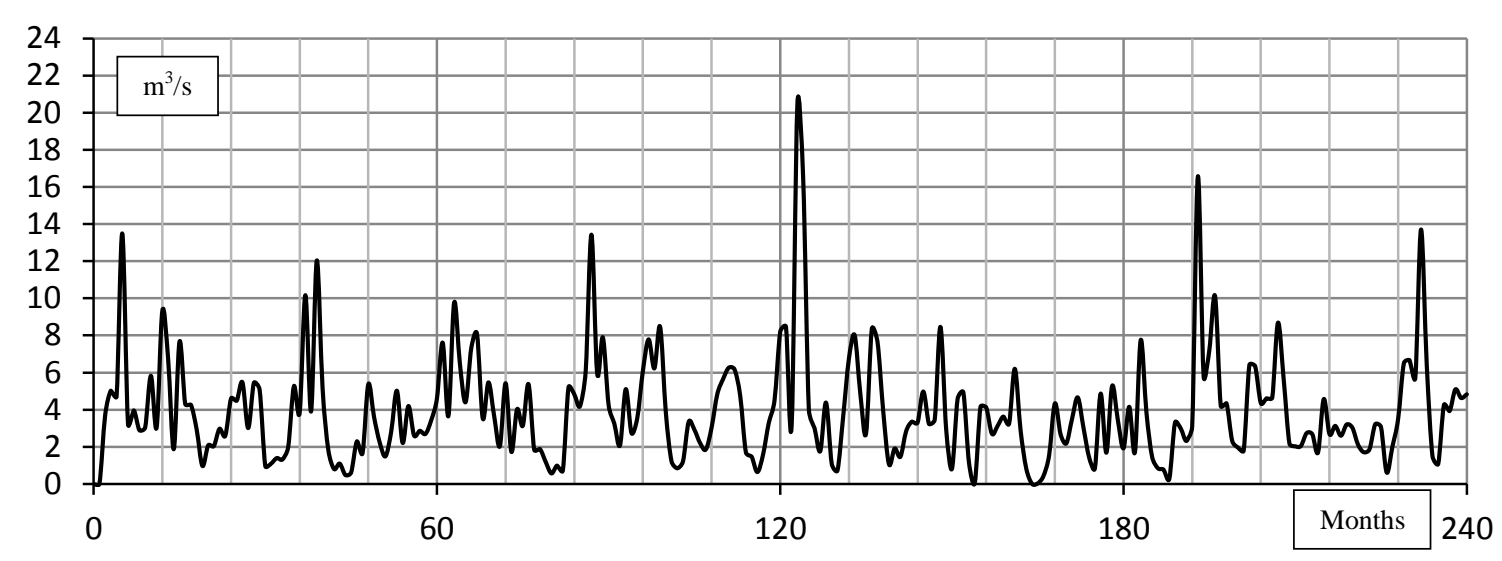

(a) Historic Climate Conditions

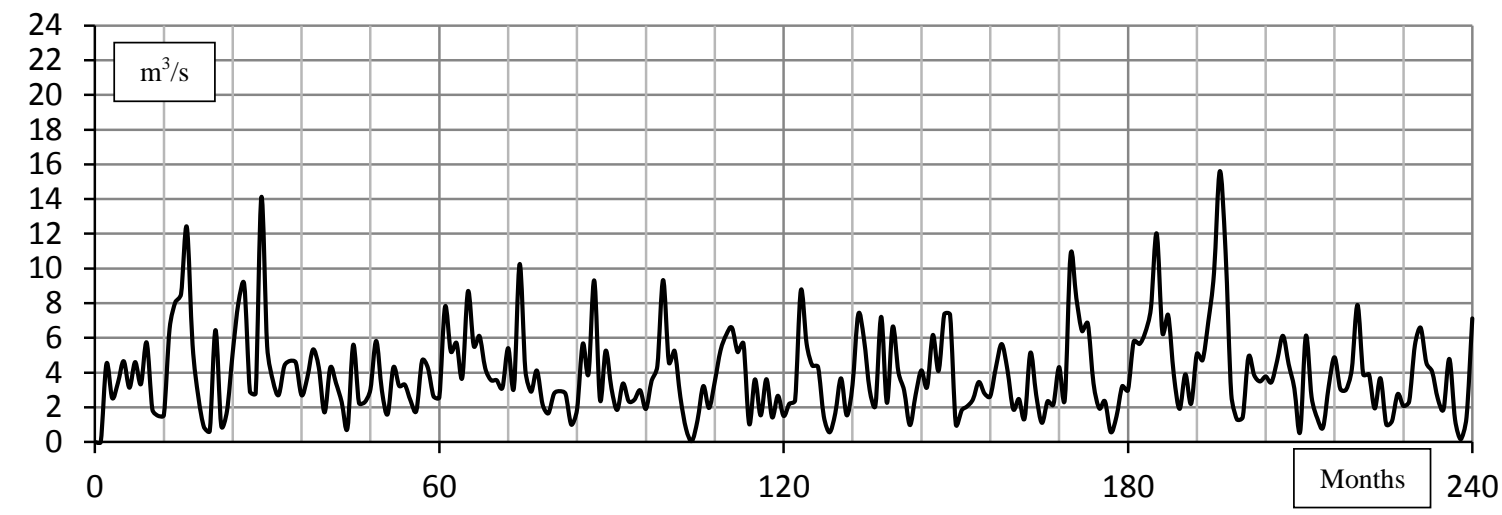

(b) Wet Climate Conditions

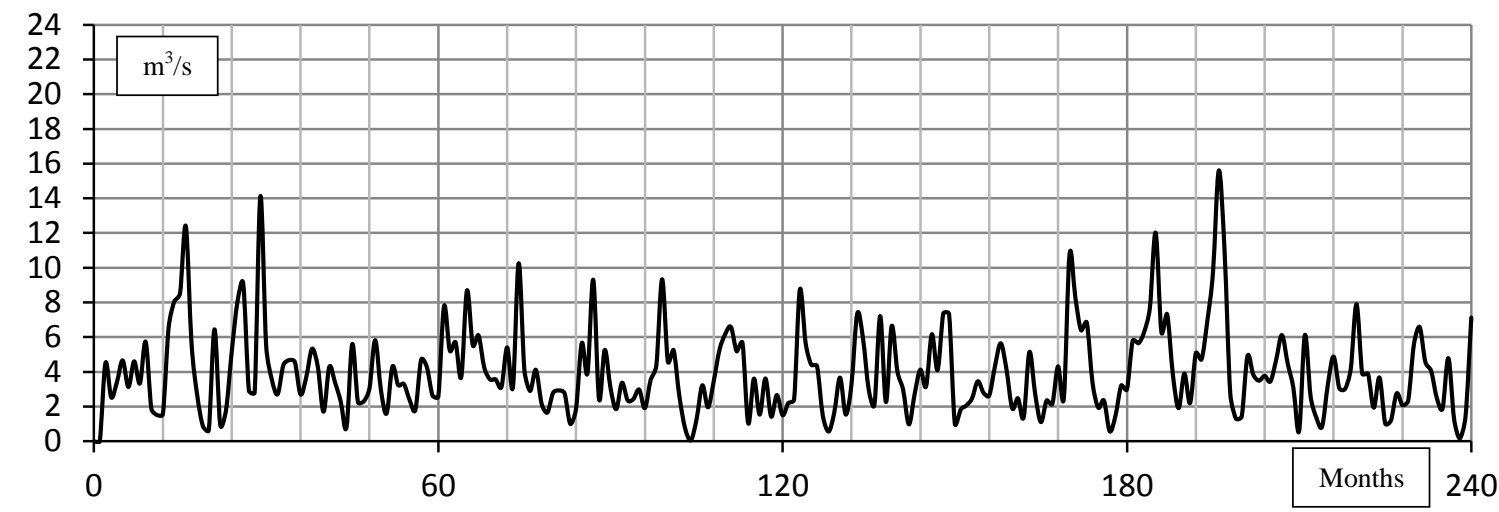

(c) Dry Climate Conditions

Figure 75: River Thames median monthly flow rates at St. Marys for three climate scenarios $(a, b, c)$ 


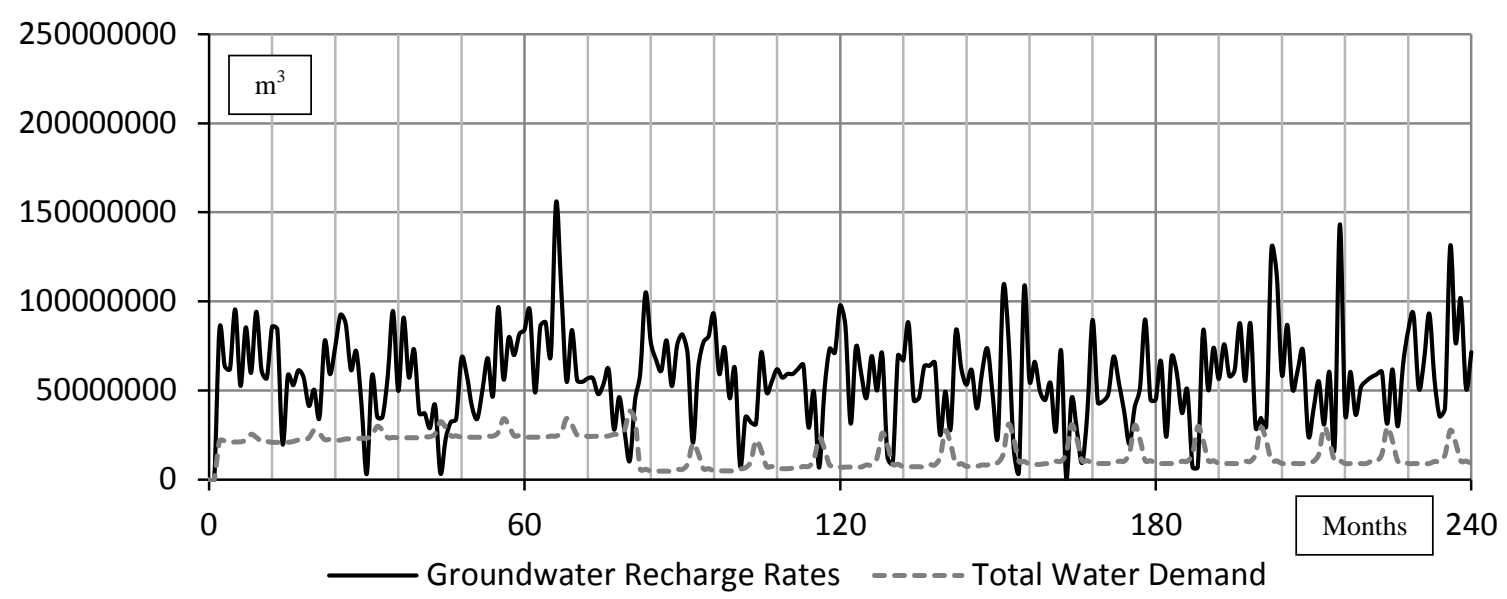

(a) Historic Climate Conditions

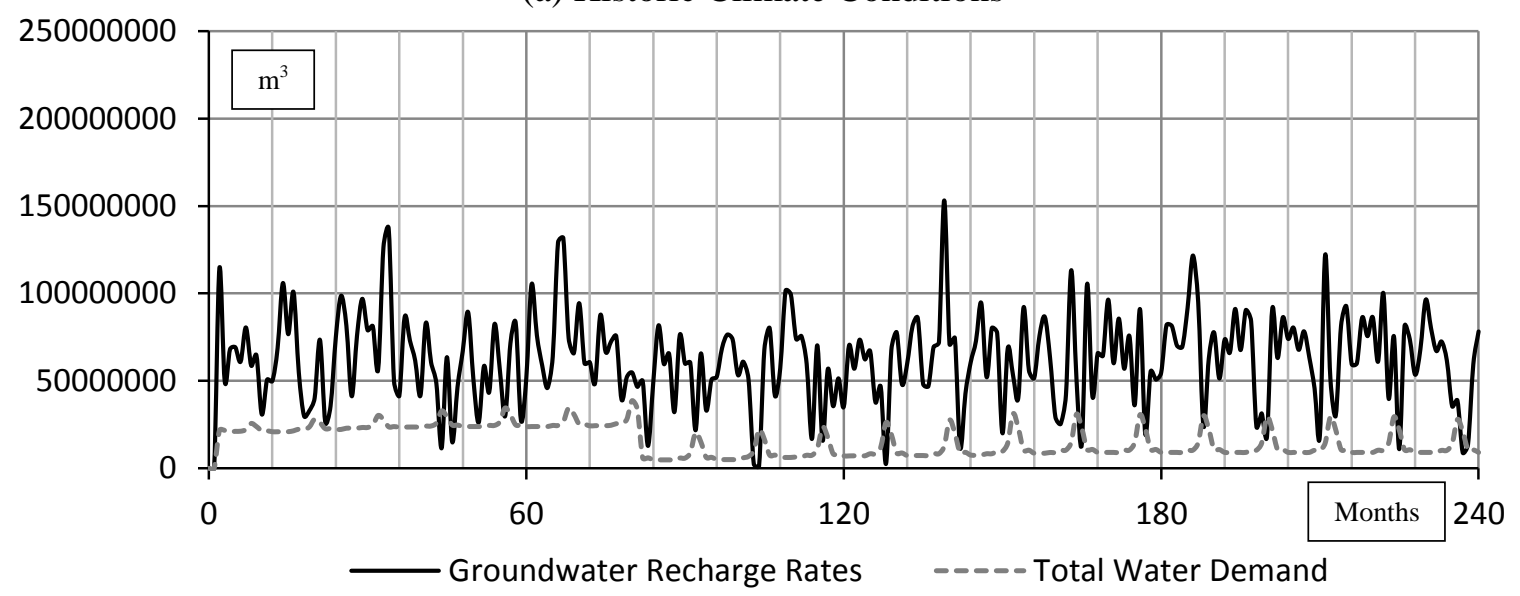

(b) Wet Climate Conditions

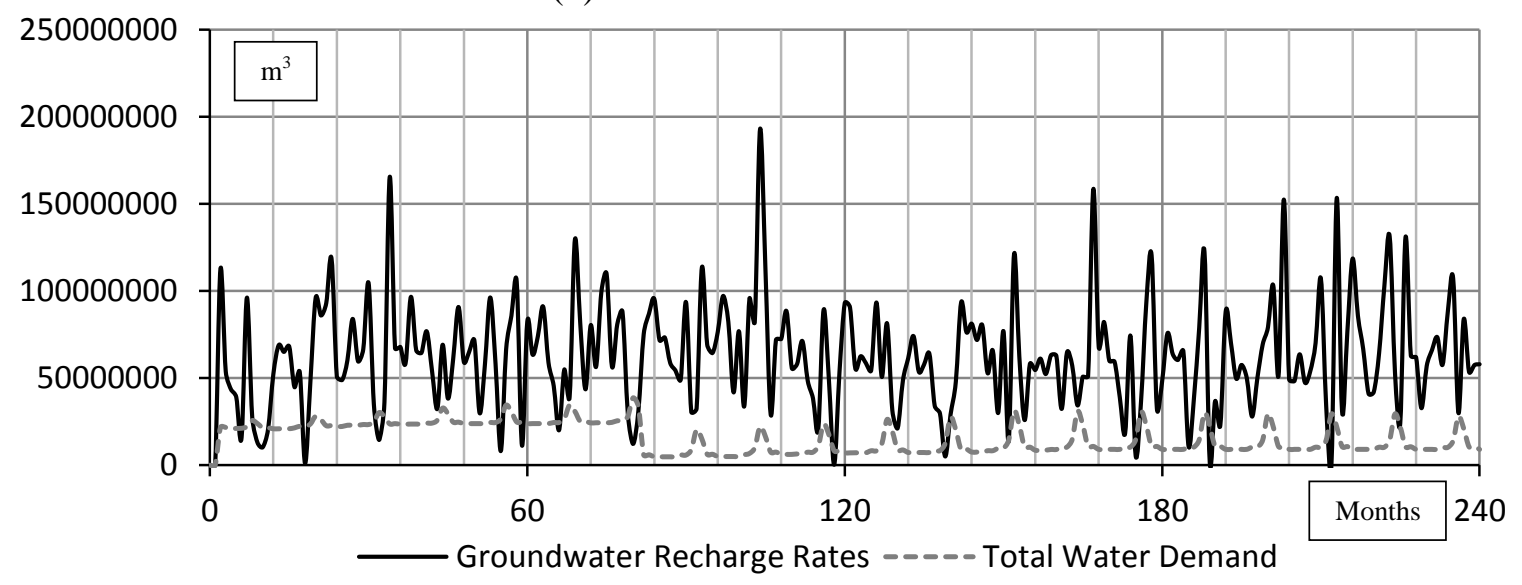

(c) Dry Climate Conditions

Figure 76: Middlesex County groundwater recharge rates and total water demand for three climate scenarios $(\mathrm{a}, \mathrm{b}, \mathrm{c})$ 


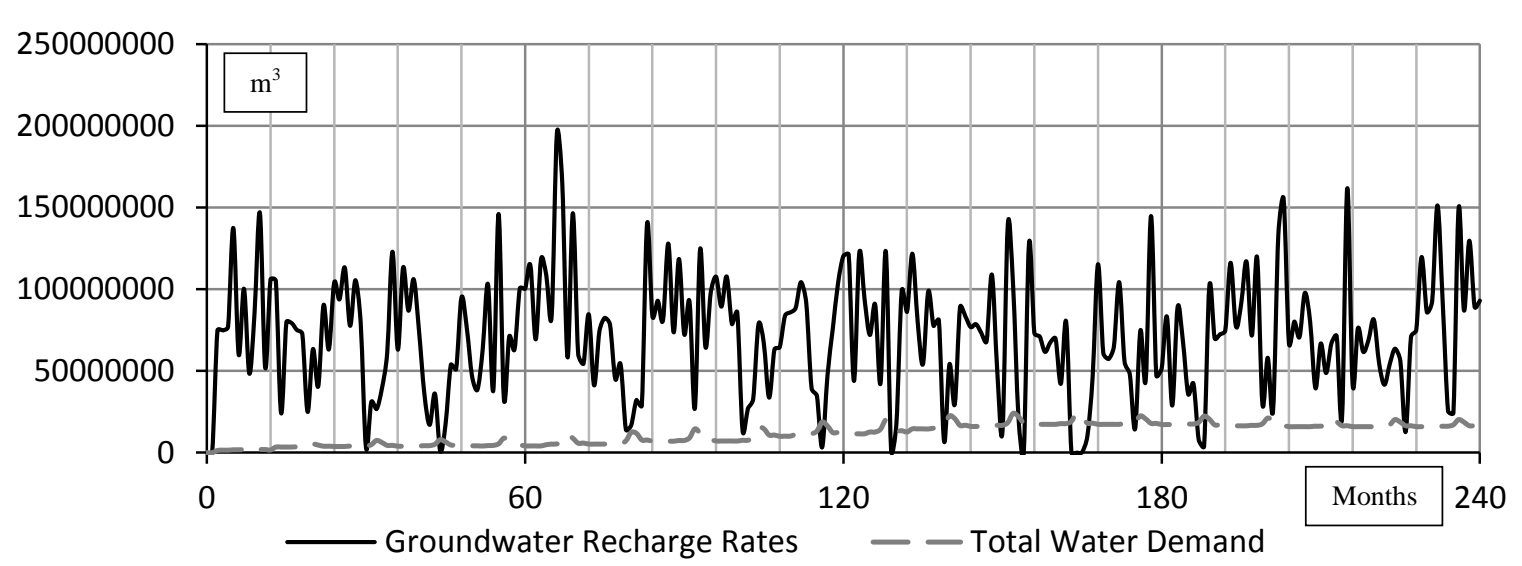

(a) Historic Climate Conditions

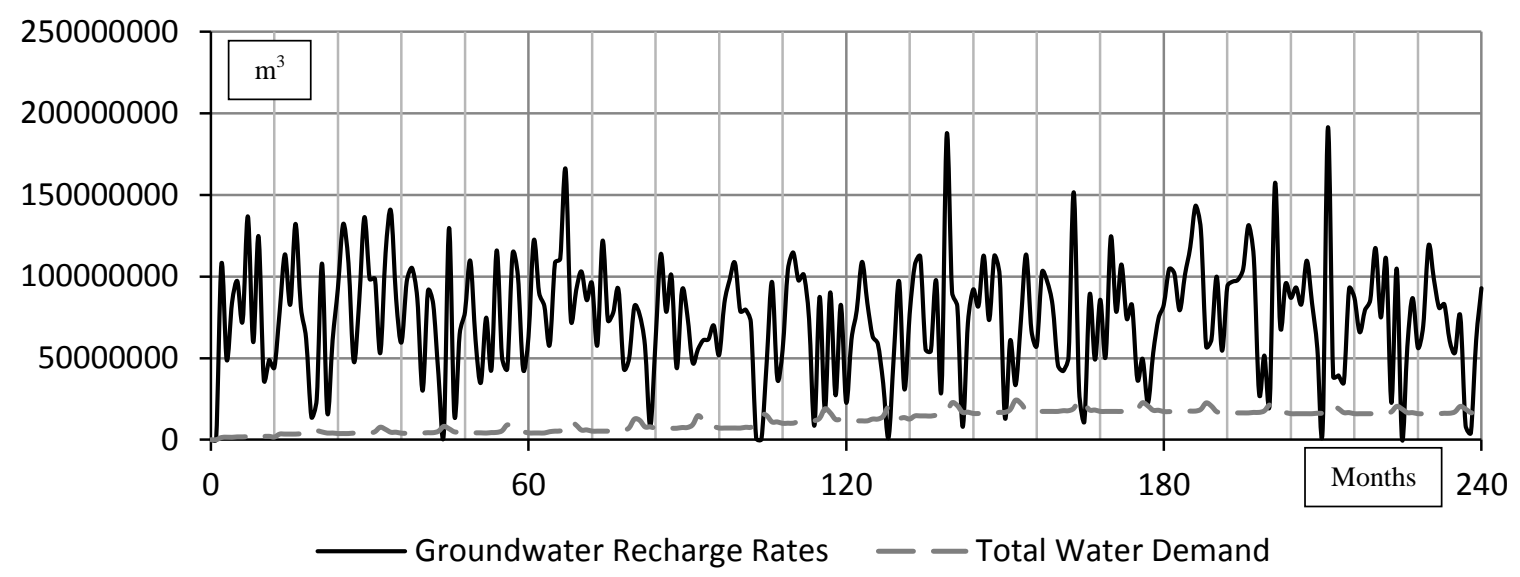

(b) Wet Climate Conditions

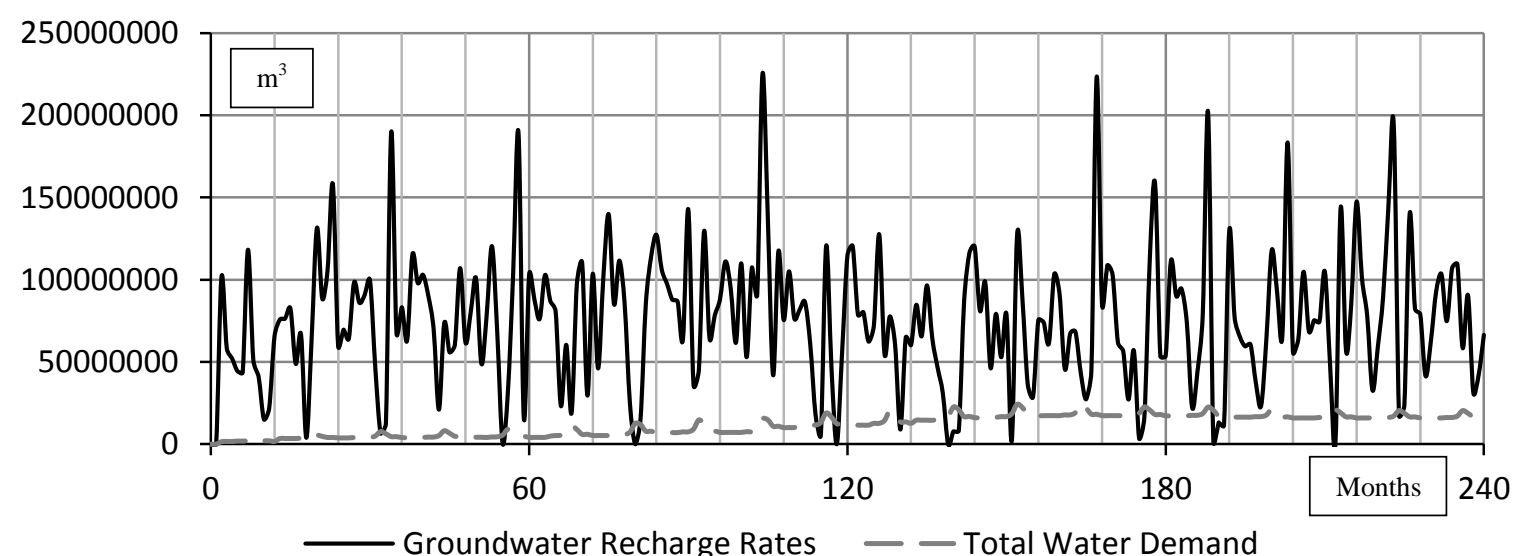

(c) Dry Climate Conditions

Figure 77: Oxford County groundwater recharge rates and total water demand for three climate scenarios $(\mathrm{a}, \mathrm{b}, \mathrm{c})$ 


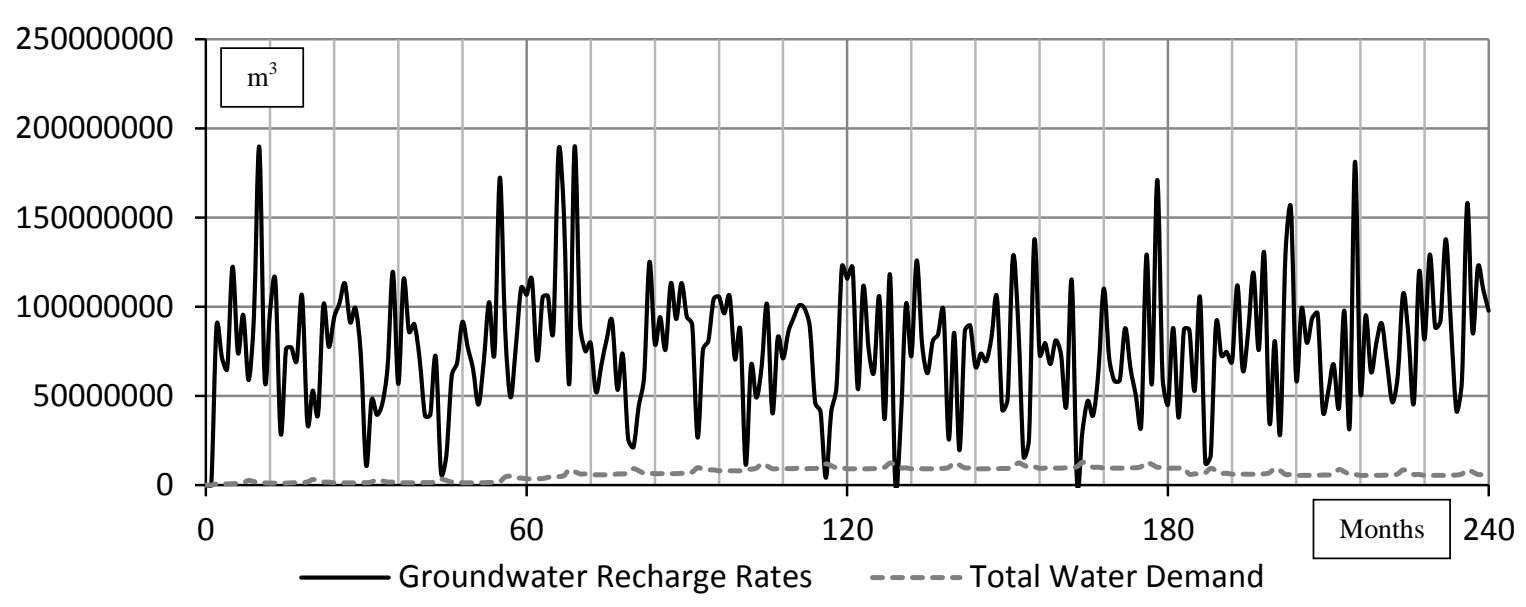

(a) Historic Climate Conditions

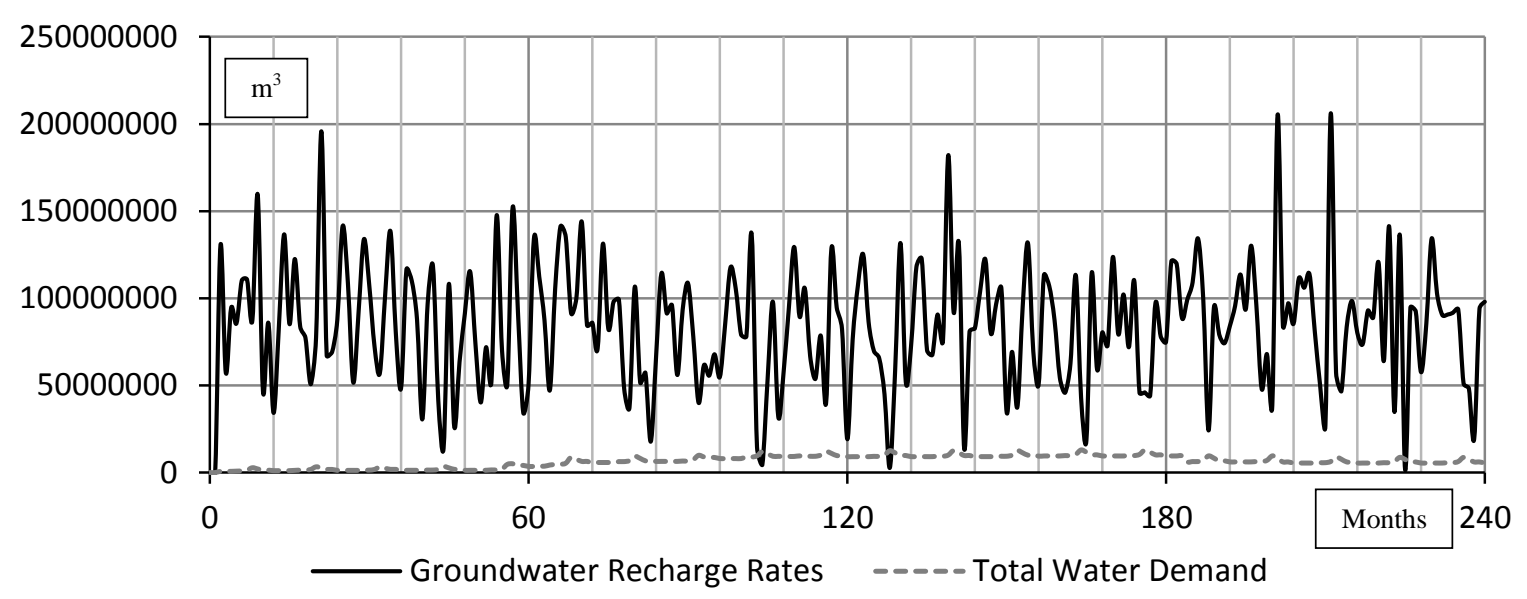

(b) Wet Climate Conditions

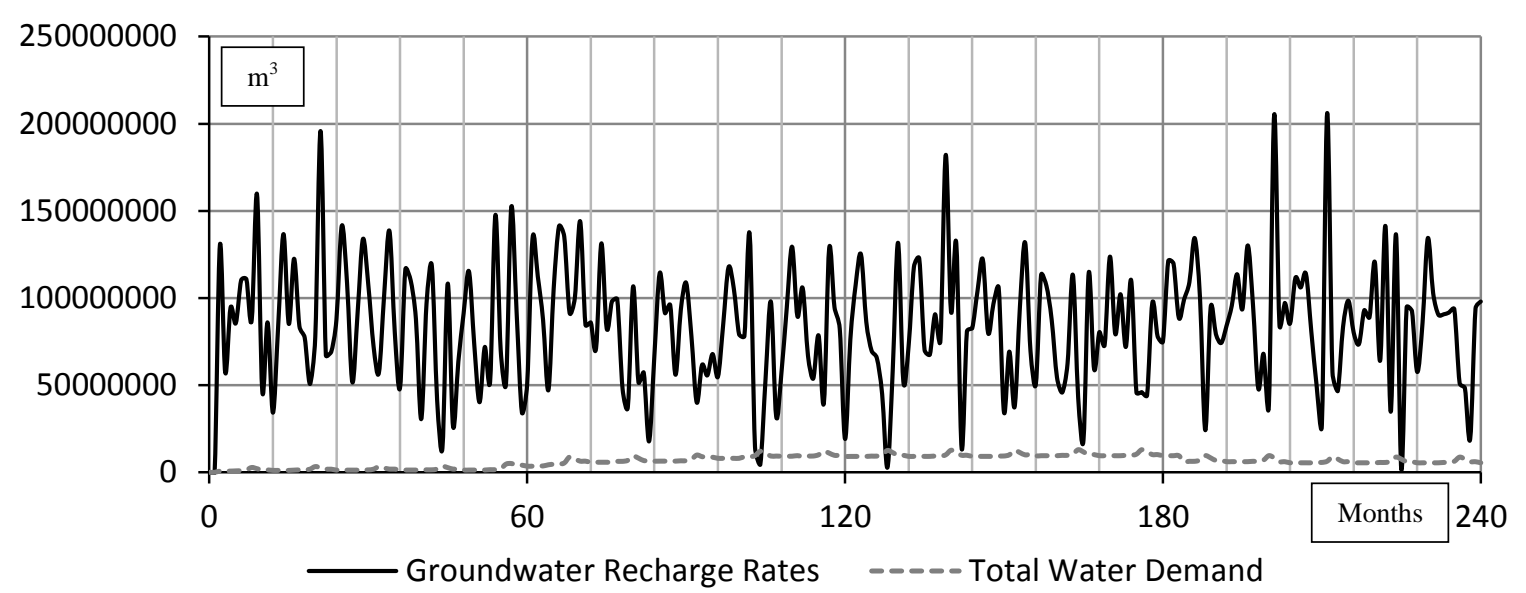

(c) Dry Climate Conditions

Figure 78: Perth County groundwater recharge rates and total water demand for three climate scenarios $(\mathrm{a}, \mathrm{b}, \mathrm{c})$ 


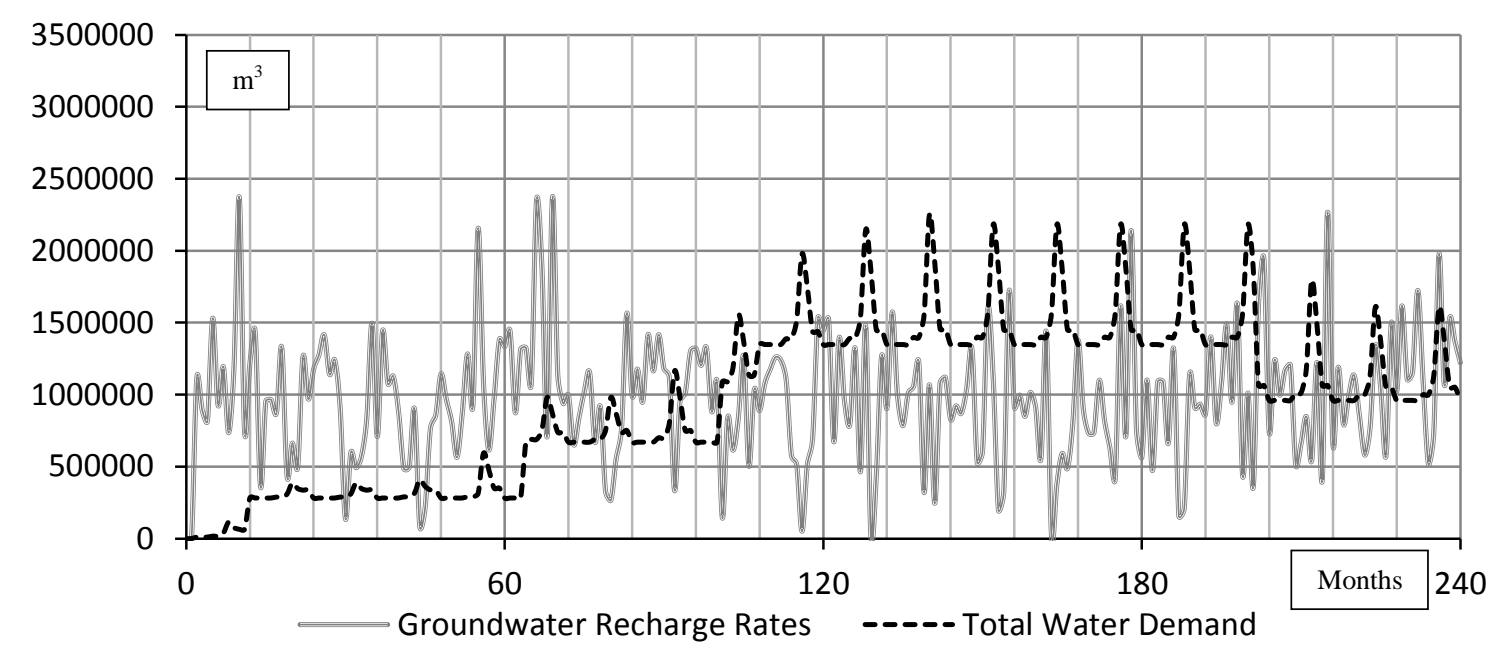

(a) Historic Climate Conditions

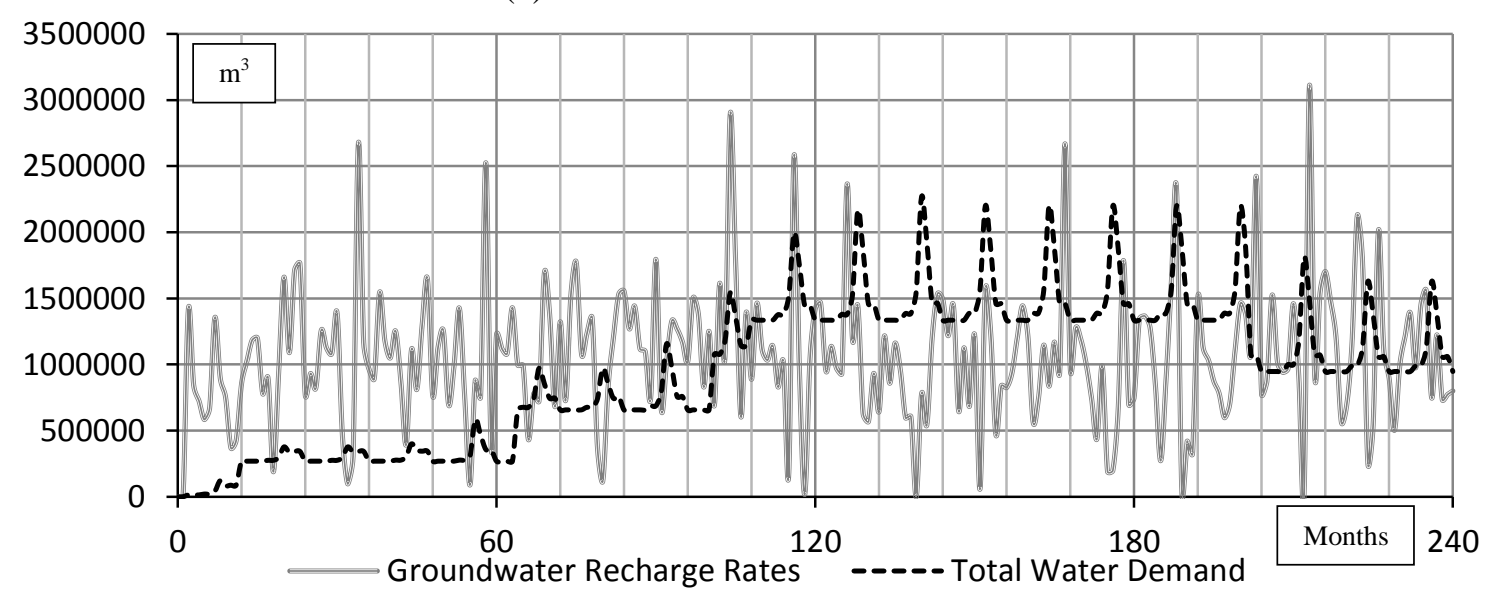

(b) Wet Climate Conditions

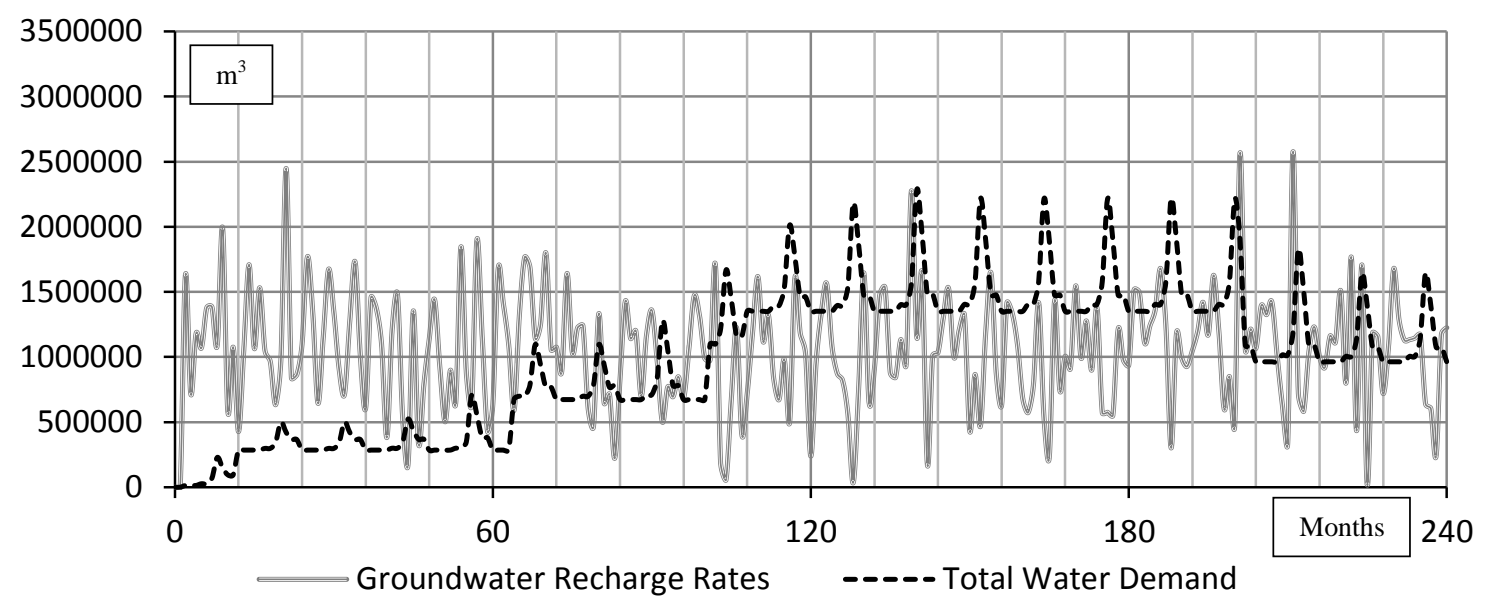

(c) Dry Climate Conditions

Figure 79: Middle Thames (sub-basin 14) groundwater recharge rates and total water demand for three climate scenarios $(\mathrm{a}, \mathrm{b}, \mathrm{c})$ 


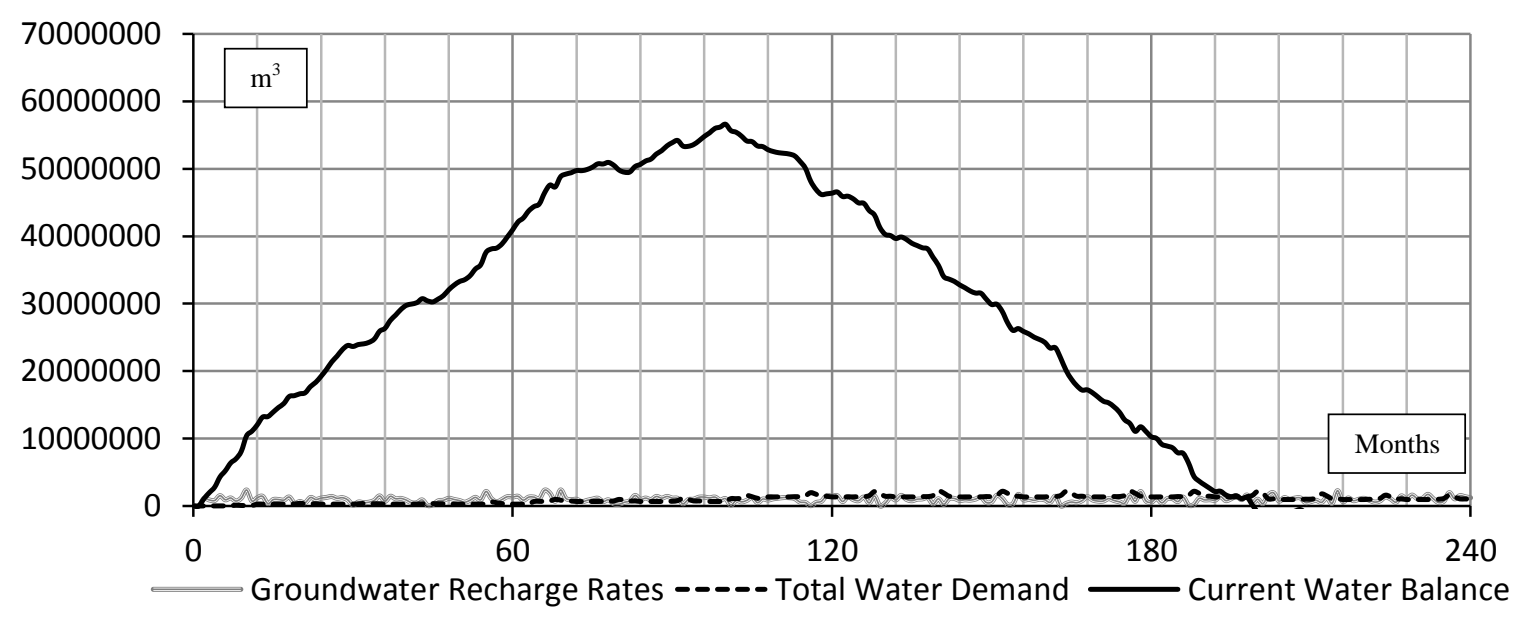

(a) Historic Climate Conditions

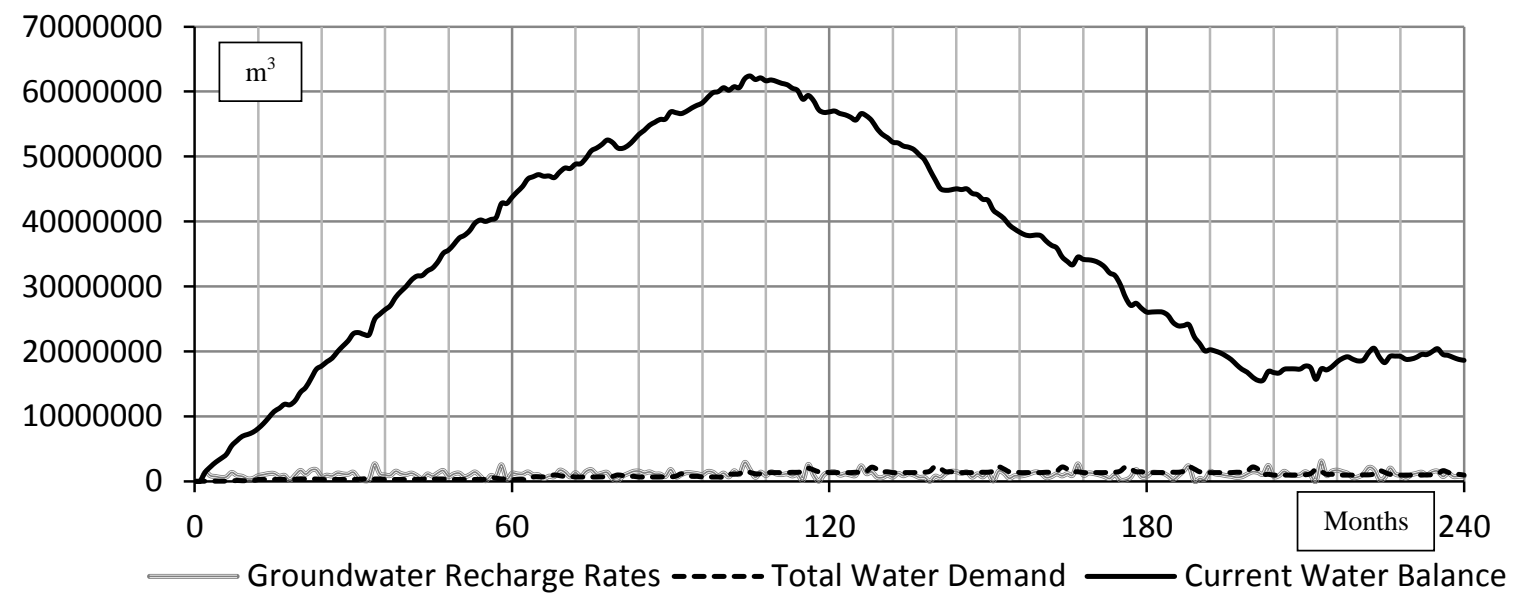

(b) Wet Climate Conditions

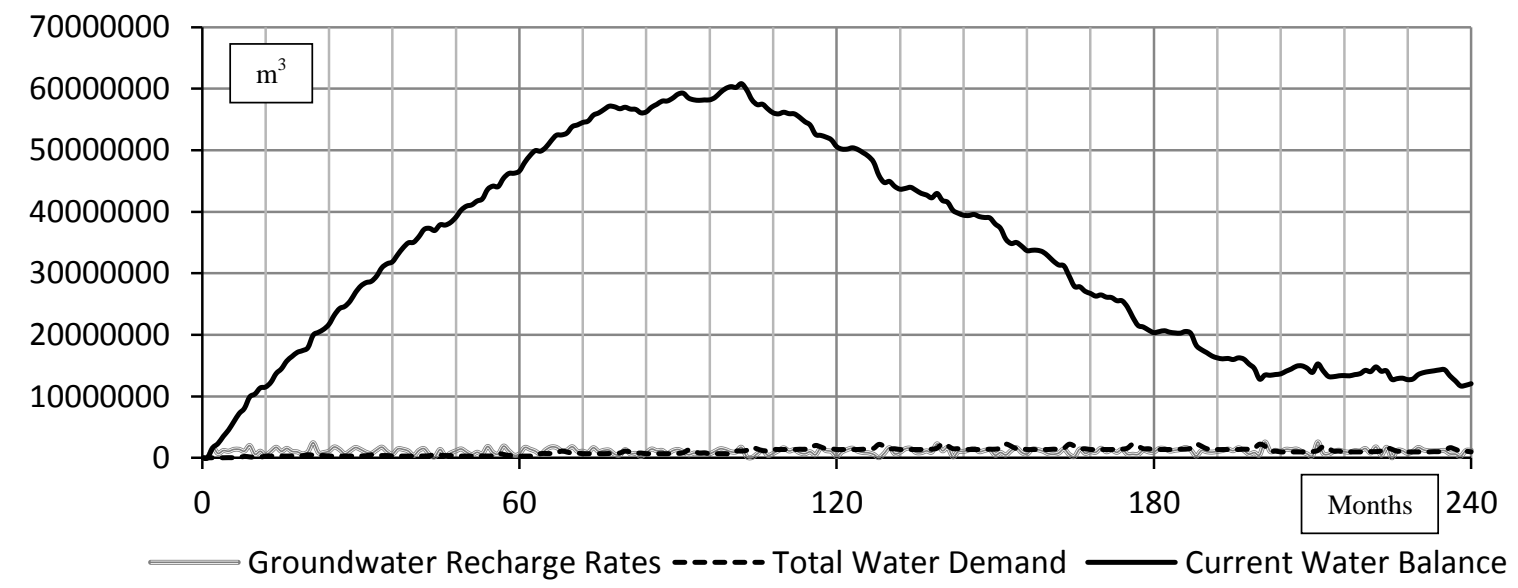

(c) Dry Climate Conditions

Figure 80: Middle Thames (sub-basin14) cumulative water balance for three climate scenarios $(\mathrm{a}, \mathrm{b}, \mathrm{c})$ 


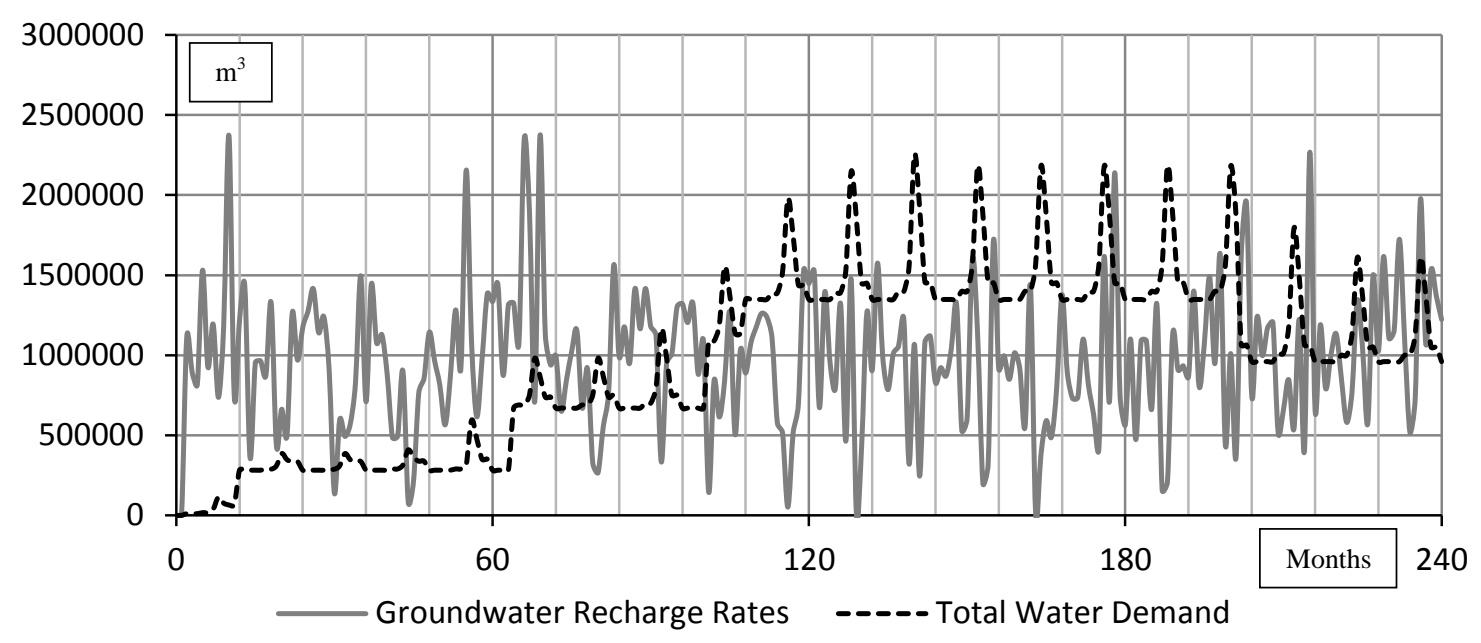

(a) Historic Climate Conditions

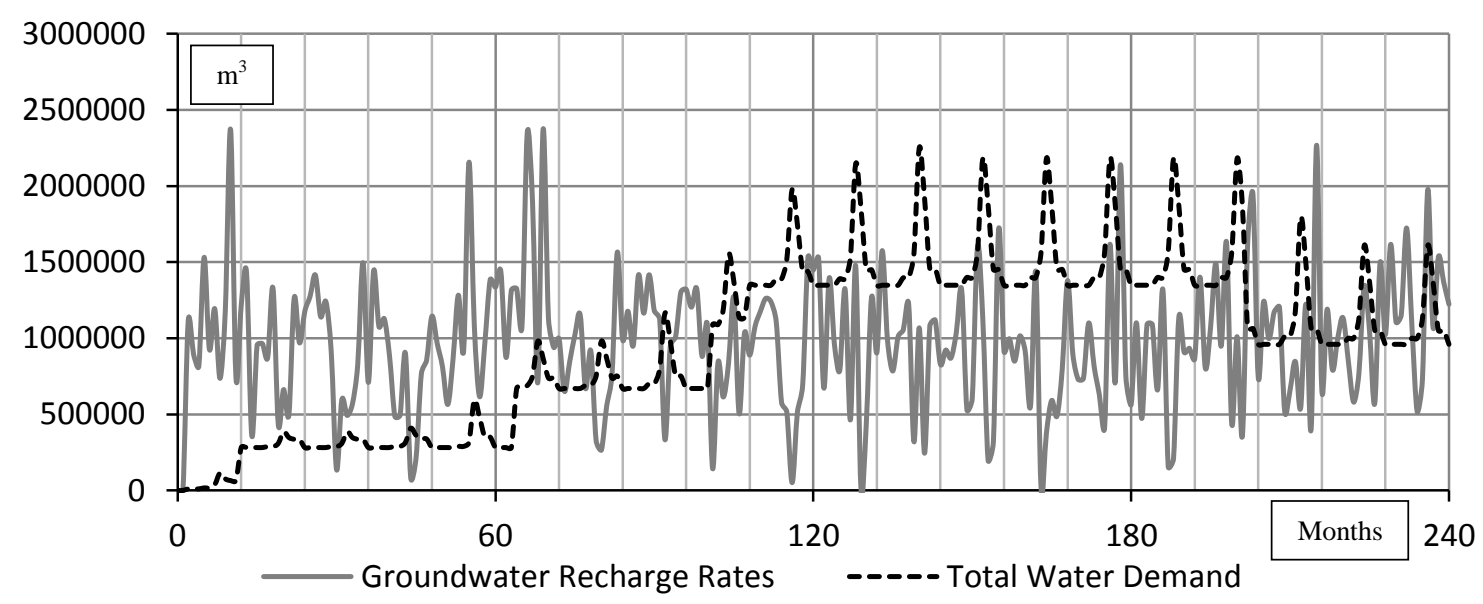

(b) Wet Climate Conditions

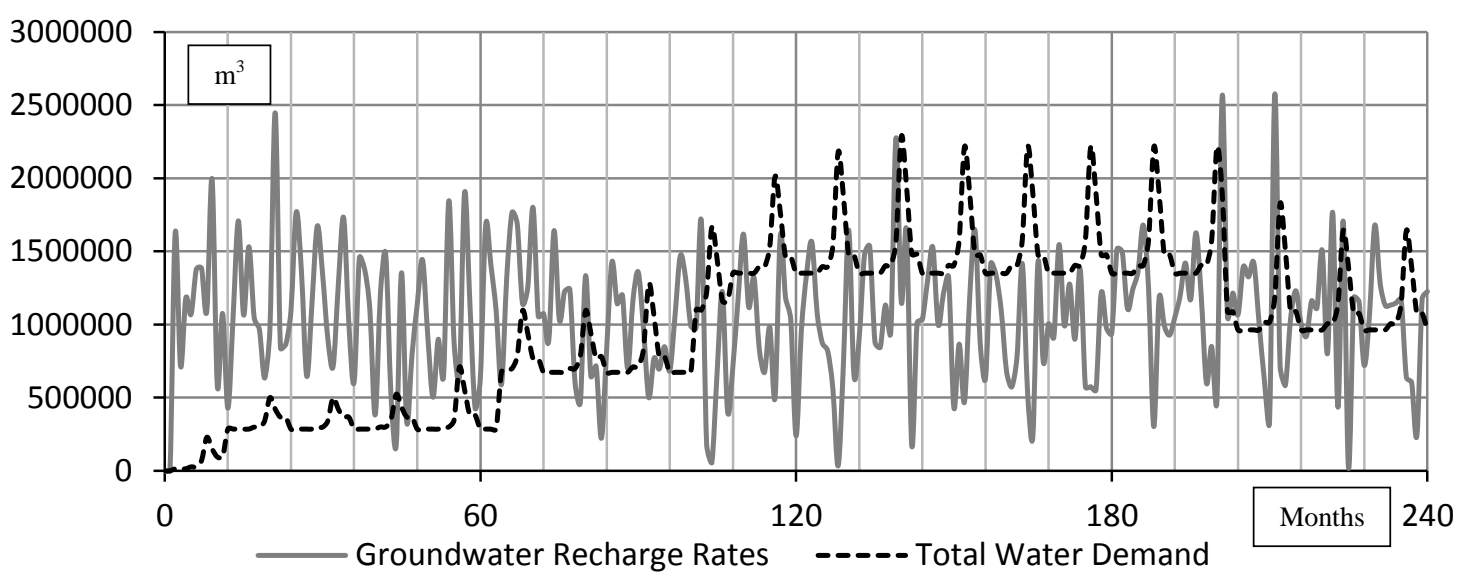

(c) Dry Climate Conditions

Figure 81: North Mitchell (sub-basin 20) groundwater recharge rates and total water demand for three climate scenarios $(\mathrm{a}, \mathrm{b}, \mathrm{c})$ 


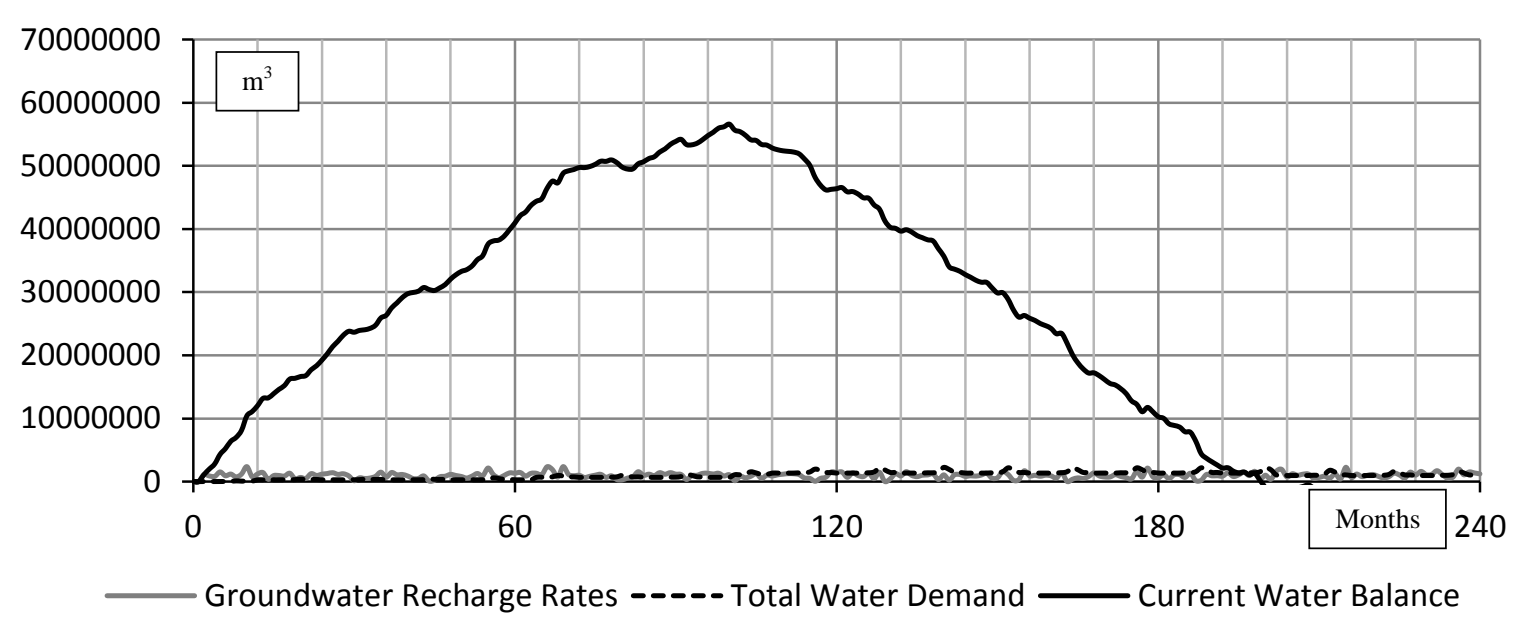

(a) Historic Climate Conditions

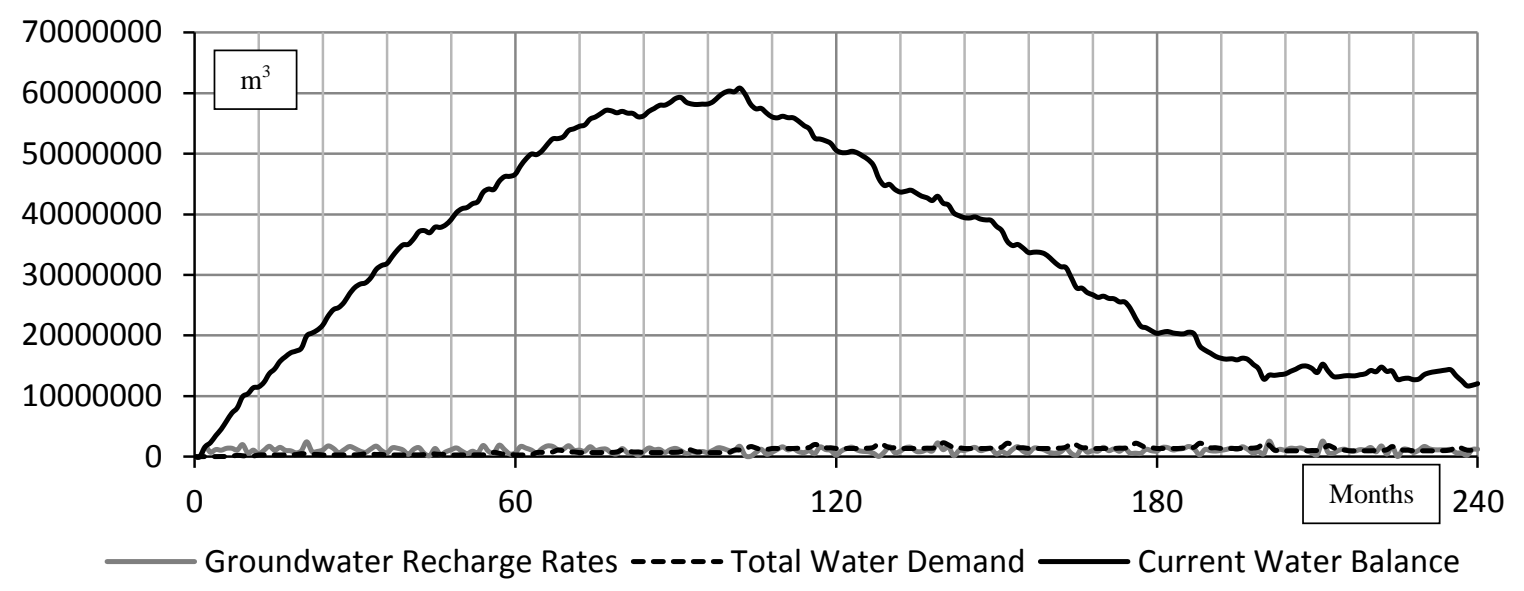

(b) Wet Climate Conditions

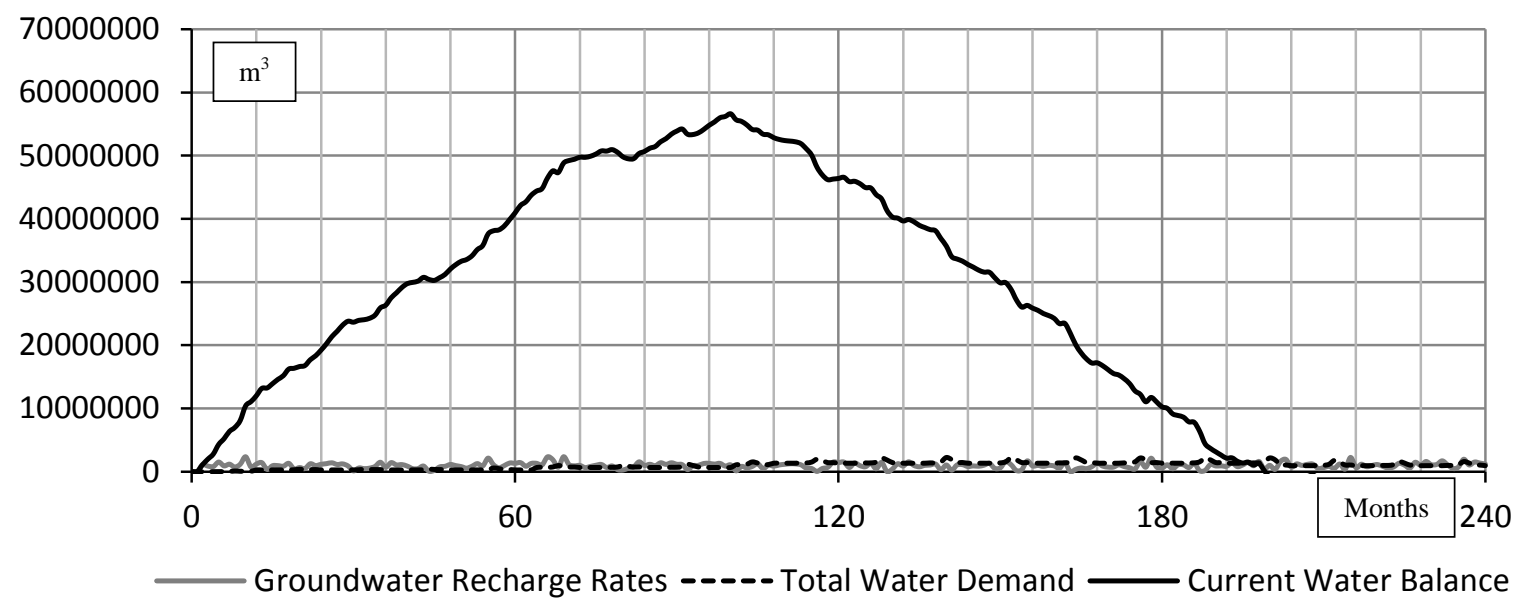

(c) Dry Climate Conditions

Figure 82: North Mitchell (sub-basin 20) cumulative water balance for three climate scenarios $(a, b, c)$ 


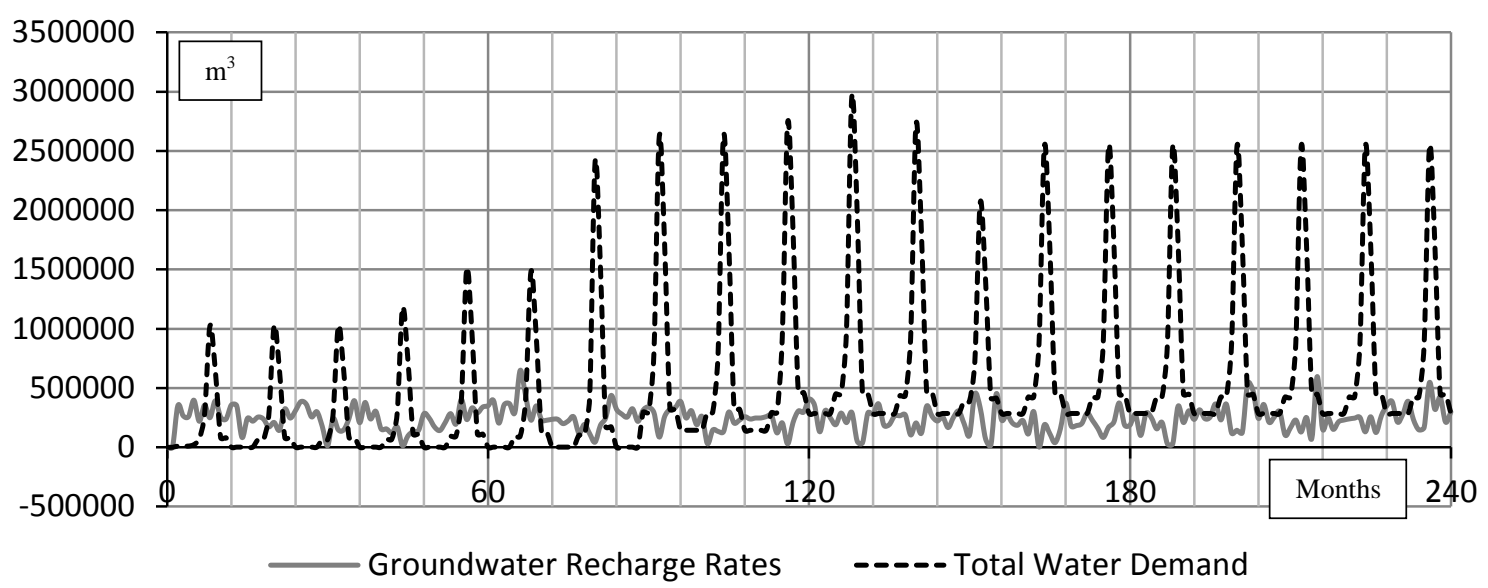

(a) Historic Climate Conditions

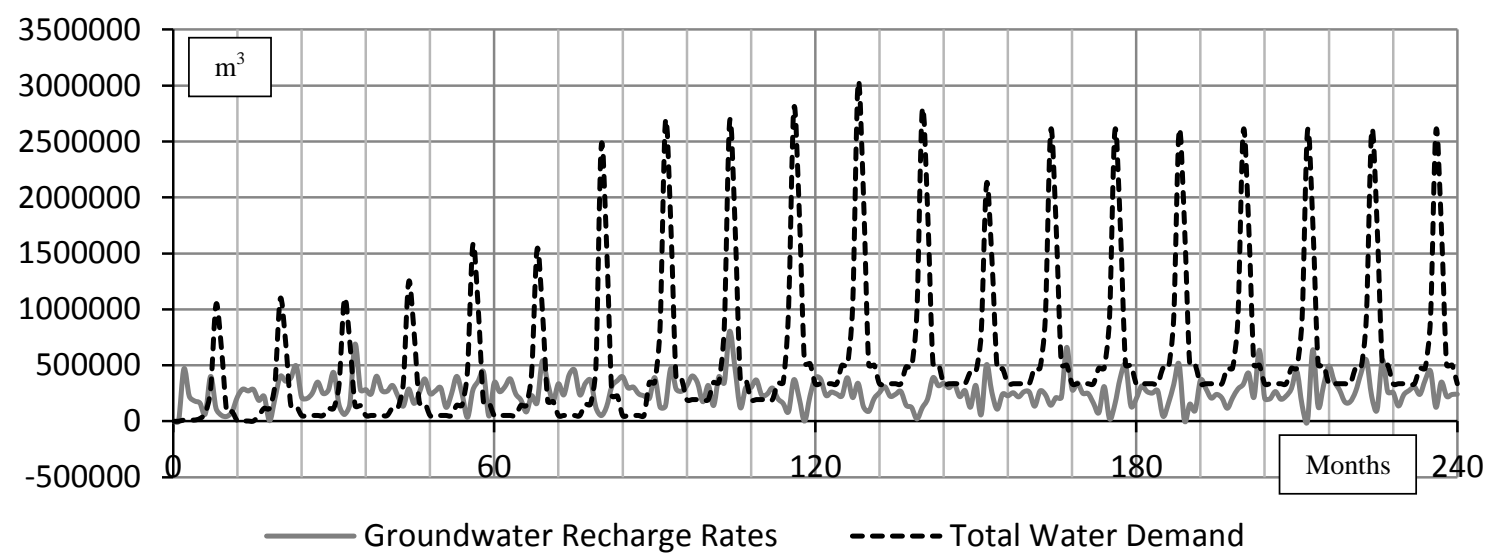

(b) Wet Climate Conditions

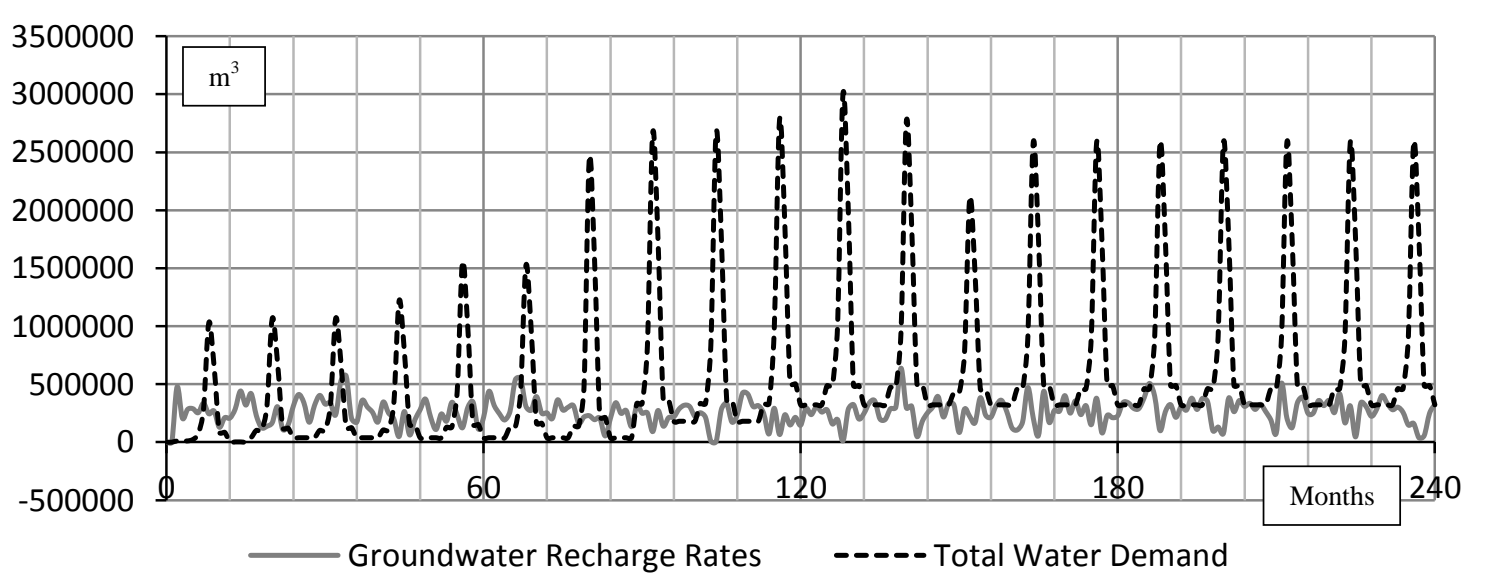

(c) Dry Climate Conditions

Figure 83: River Bend (sub-basin 27) groundwater recharge rates and total water demand for three climate scenarios $(a, b, c)$ 


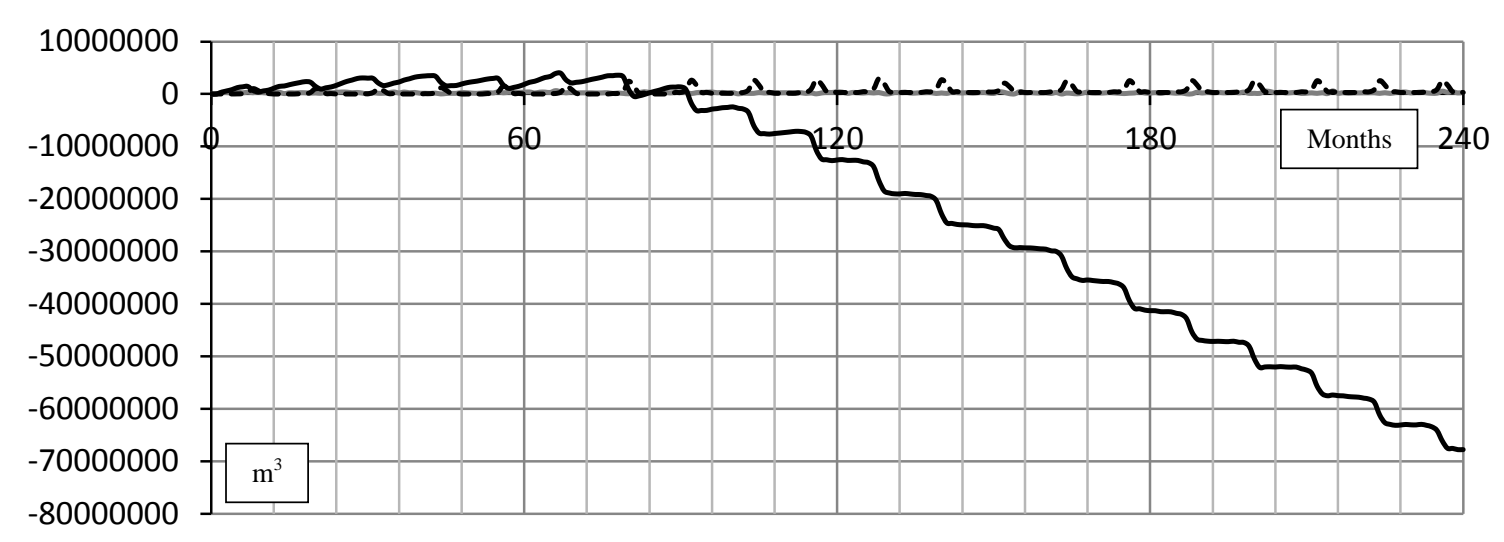

Groundwater Recharge Rates ----- Total Water Demand —Current Water Balance

(a) Historic Climate Conditions

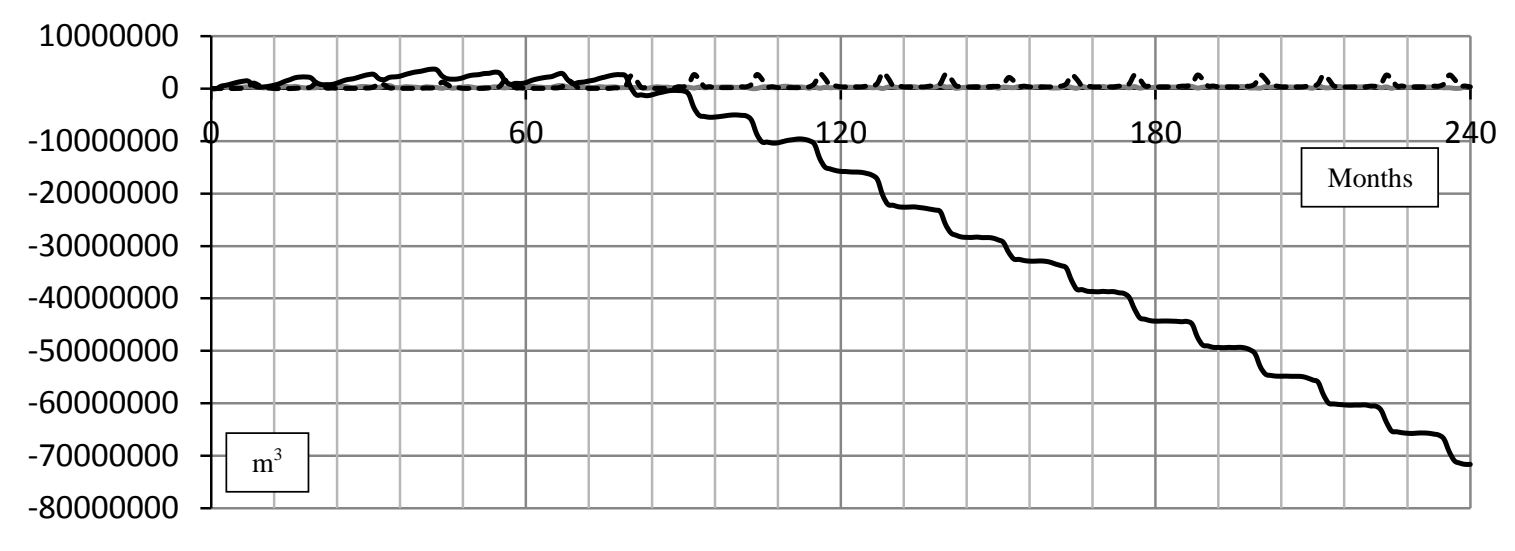

Groundwater Recharge Rates ----- Total Water Demand —C Current Water Balance

(b) Wet Climate Conditions

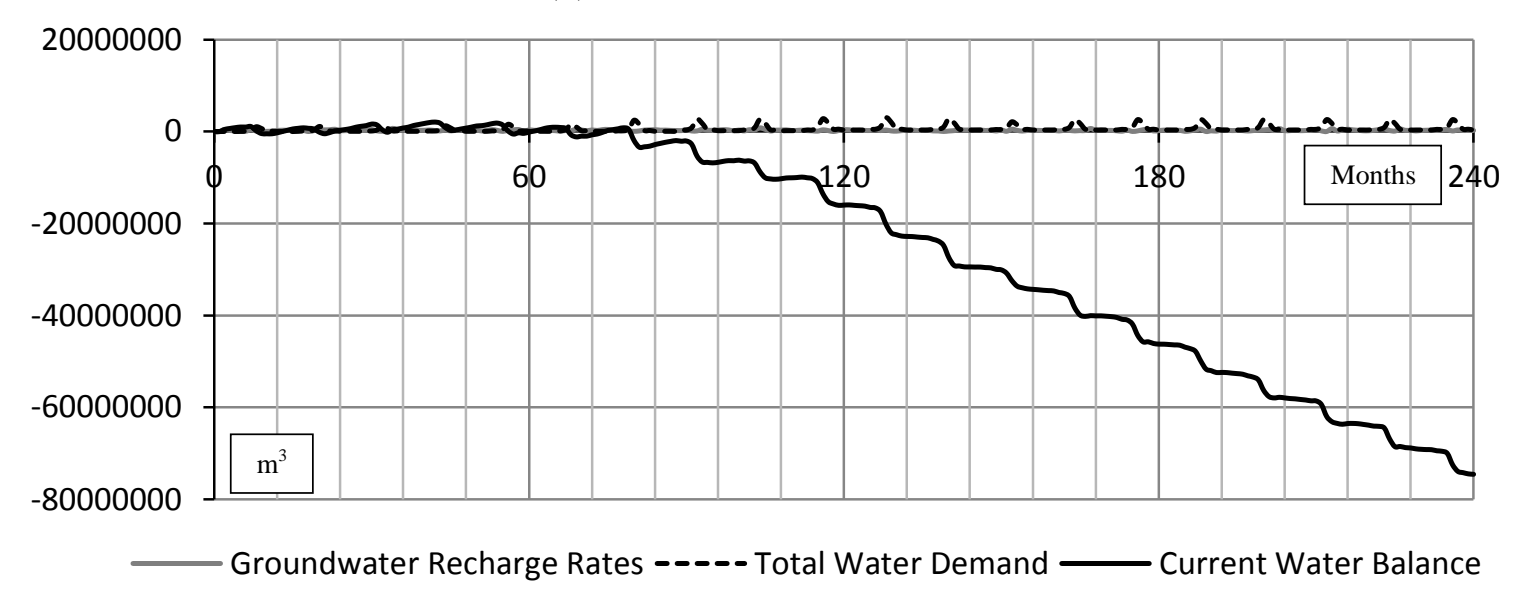

(c) Dry Climate Conditions

Figure 84: River Bend (sub-basin 27) cumulative water balance for three climate scenarios $(a, b, c)$ 


\subsection{Changes in the local hydrologic regime}

One of the main questions that spatially explicit agent-based model is designed to address is how changes caused by socio-economic activities affect the hydrologic cycle in the Upper Thames River basin. Arnel (2002) divides human-induced changes in the basin into three main categories. Land-cover changes (deforestation, afforestation, agriculture, urbanization, and mining) incorporate changes to the vegetation covering the basin. The second category of changes refers to the use and exploitation of the water in the basin (municipal and domestic, industry, agriculture, impoundment and transfer). Finally, the third describes physical changes to the river network. According to Arnel (2002), these three categories of changes have three types of effects on the quantity and quality of water resources in the river basin - effects on the volume of flow and groundwater storage, effects on the timing of flow, effects on the quality of water in soils, rivers and groundwater. Therefore, this model analyzes one of the most significant implications of land-use change and urbanization on hydrologic regime - alteration of surface water flow regime and groundwater recharge rates. Tables 31, 33 and 35 summarize resulting median and average values for three climate stations and six combinations of socioeconomic and climate scenarios. In addition, Tables 32, 34 and 36 show the total volumes of runoff at three flow gauging stations (Byron, Ingersoll and St. Marys) during the course of simulation (20 years) for all three climate conditions (a, b, and c) and two socio-economic scenarios. The presented results presented reveal two important characteristics of the system's hydrologic behavior.

First, the initial assumption is that the higher rates of urbanization are influencing the groundwater recharge rates since a larger portion of rainfall ends up in rivers and streams, thus increasing the volumes of surface runoff. As expected, the different climate conditions demonstrate the variability in volumes, but, more importantly, the results show the increased volumes of surface runoff in the case of infinite natural resources scenario. For instance, in the case of historic climate conditions, the Byron station experiences $4.76 \%$ higher runoff volumes as a response to more intensive urbanization process compared to the baseline socio-economic scenario, Table 32. The same trends are observed for other two stations, Ingersoll and St. Marys, Table 34 and 36.These findings 
are in agreement with the analysis done by UK National Ecosystem Assessment and their report on the effects of future land-cover change on UK River flows, Gosling (2013). This analysis uses spatially distributed hydrologic model to analyze the effects of different land-cover change scenarios on river flows in 34 UK basins. Three hydrologic indicators are considered: average annual discharge, high and low flows, flood hazards. Six UK National Ecosystem Assessment (NEA) scenarios describe how ecosystem services and human well-being might change under a range of possible futures. These scenarios present possible social and economic conditions in 2060. Also, the analysis examines the two most extreme future climate conditions - wet and dry. 34 selected basins vary in sizes (ranging $\left.9-1363 \mathrm{~km}^{2}\right)$, mean daily river flow $\left(0.5-23.6 \mathrm{~m}^{3} / \mathrm{s}\right)$ and elevation $(39-496 m)$. The results reveal that land-cover change has a more significant effect on the extremes (high and low flows) than on average annual discharge. For annual discharge, the range across all scenarios and river basins varies between $-13 \%$ and $+6 \%$. Expectedly, these variations strongly depend on the applied socio-economic scenario and resulting land cover changes.

The presented results do not show a significant variability of mean runoff between different climate scenarios as observed in previous studies. The reason for that lies in the choice of initial climate datasets. Datasets used in this research were originally created to define three different climate conditions in the period between 2000 and 2100. On the other hand, available socio-economic data requested that the socio-economic model is developed for the period between 2000 and 2020 with a monthly time step. Analysis of the first 20 years of precipitation input for London Airport CS does not show the significant variability between three climate scenarios, Figure 85 . This seems to be the main reason why significant variability is not present in hydrologic system response. One more model assumption has an impact on obtained results - defined operational policies for three reservoirs. 


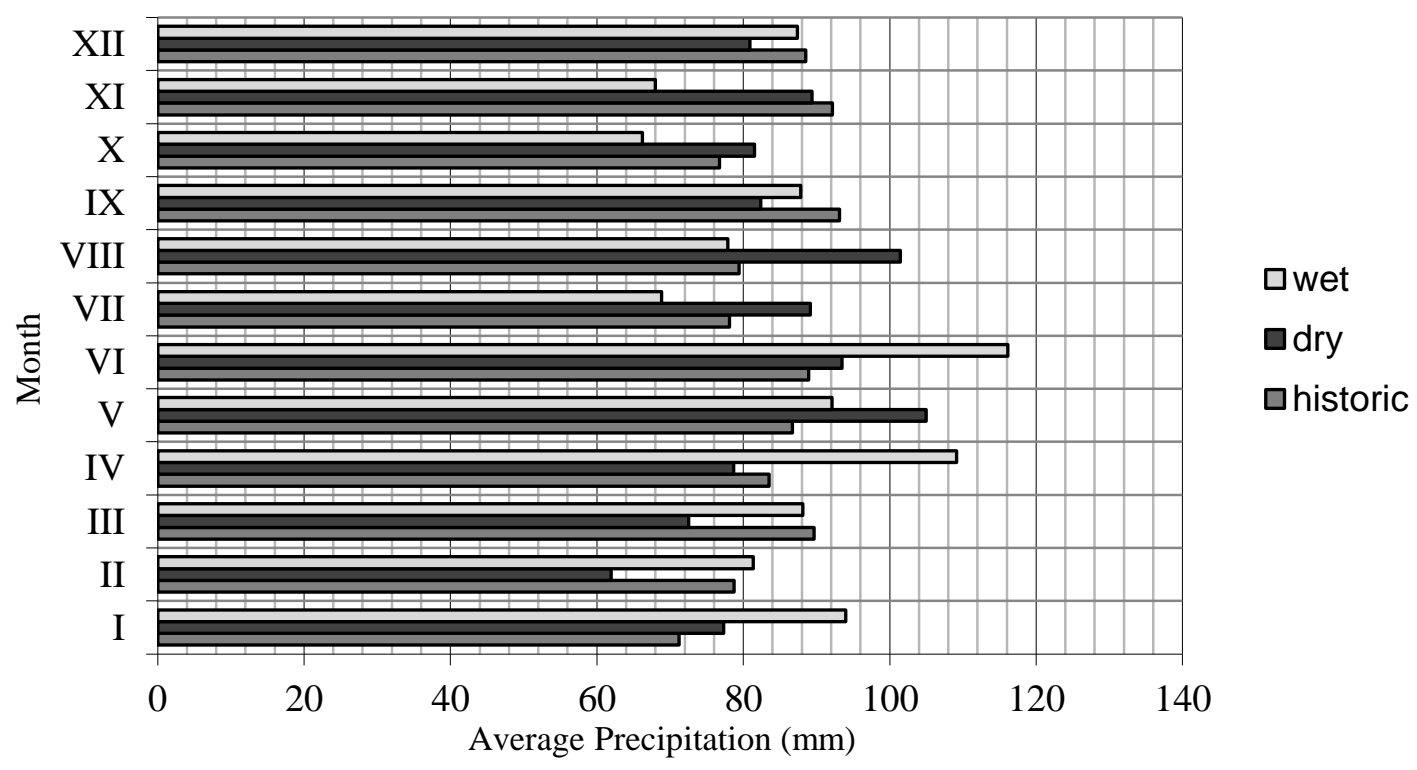

Figure 85: Monthly distribution of precipitation for three climate scenarios for London

\section{Airport CS}

Since a larger portion of precipitation ends up in rivers and streams, less water is infiltrated into the groundwater aquifers that many municipalities use for their drinking water supply and other socio-economic activities. This is directly observed in Tables 33, 34 , and 35 which show the lower groundwater recharge rates in the case of infinite natural resources scenario and all three climate conditions. These trends are identical to the ones obtained for the surface runoff. 
Table 31: Median and average monthly flows at Byron station $\left[\mathrm{m}^{3} / \mathrm{s}\right]$

\begin{tabular}{ccccc}
\hline \multirow{2}{*}{ Byron } & \multicolumn{2}{c}{ Baseline SE } & \multicolumn{2}{c}{ Infinite SE } \\
\cline { 2 - 5 } Station & Median & Average & Median & Average \\
\hline Historic & 25.46 & 30.02 & 26.52 & 31.45 \\
Wet & 27.84 & 33.25 & 29.45 & 35.02 \\
Dry & 25.62 & 27.65 & 26.35 & 28.51 \\
\hline
\end{tabular}

Table 32: Changes in total runoff volumes calculated for 20 years at Byron station $\left[\mathrm{m}^{3}\right]$

\begin{tabular}{ccccc}
\hline Byron & & Baseline SE & Infinite SE & Change \\
\cline { 3 - 5 } Station & & (i) & (ii) & {$[\%]$} \\
\hline Historic Climate & (a) & $1.895 \mathrm{E}+10$ & $1.985 \mathrm{E}+10$ & $+4.76 \%$ \\
Wet Climate & (b) & $2.099 \mathrm{E}+10$ & $2.210 \mathrm{E}+10$ & $+5.32 \%$ \\
Dry Climate & (c) & $1.745 \mathrm{E}+10$ & $1.799 \mathrm{E}+10$ & $+3.11 \%$ \\
\hline Change (b)-(a) & {$[\%]$} & $+10.76 \%$ & $+11.35 \%$ & \\
Change (c)-(a) & {$[\%]$} & $-7.89 \%$ & $-9.34 \%$ & \\
\hline
\end{tabular}


Table 33: Median and average monthly flows at Ingersoll station $\left[\mathrm{m}^{3} / \mathrm{s}\right]$

\begin{tabular}{ccccc}
\hline Ingersoll & \multicolumn{2}{c}{ Baseline SE } & \multicolumn{2}{c}{ Infinite SE } \\
\cline { 2 - 5 } Station & Median & Average & Median & Average \\
\hline Historic & 9.33 & 11.17 & 9.95 & 12.05 \\
Wet & 9.88 & 12.05 & 10.88 & 14.11 \\
Dry & 8.35 & 10.75 & 9.83 & 11.32 \\
\hline
\end{tabular}

Table 34: Changes in total runoff volumes calculated for 20 years at Ingersoll station $\left[\mathrm{m}^{3}\right]$

\begin{tabular}{ccccc}
\hline $\begin{array}{c}\text { Ingersoll } \\
\text { Station }\end{array}$ & & Baseline SE & Infinite SE & Change \\
\cline { 3 - 5 } & & (i) & (ii) & {$[\%]$} \\
\hline Historic Climate & (a) & $7.050 \mathrm{E}+09$ & $7.605 \mathrm{E}+09$ & $+7.87 \%$ \\
Wet Climate & (b) & $7.605 \mathrm{E}+09$ & $8.905 \mathrm{E}+09$ & $+17.09 \%$ \\
Dry Climate & (c) & $6.785 \mathrm{E}+09$ & $7.144 \mathrm{E}+09$ & $+5.30 \%$ \\
\hline Change (b)-(a) & {$[\%]$} & $+7.87 \%$ & $+17.09 \%$ & \\
Change (c)-(a) & {$[\%]$} & $-3.76 \%$ & $-6.05 \%$ & \\
\hline
\end{tabular}


Table 35: Median and average monthly flows at St.Marys station $\left[\mathrm{m}^{3} / \mathrm{s}\right]$

\begin{tabular}{ccccc}
\hline \multirow{2}{*}{ St.Marys } & \multicolumn{2}{c}{ Baseline SE } & \multicolumn{2}{c}{ Infinite SE } \\
\cline { 2 - 5 } Station & Median & Average & Median & Average \\
\hline Historic & 3.3 & 4.01 & 3.62 & 4.24 \\
Wet & 3.49 & 4.58 & 3.95 & 4.94 \\
Dry & 3.02 & 3.61 & 3.41 & 3.94 \\
\hline
\end{tabular}

Table 36: Changes in total runoff volumes calculated for 20 years at St.Marys station

\begin{tabular}{ccccc}
\multicolumn{5}{c}{$\left[\mathrm{m}^{3}\right]$} \\
$\begin{array}{c}\text { St.Marys } \\
\text { Station }\end{array}$ & & Baseline SE & Infinite SE & Change \\
\cline { 3 - 5 } & (i) & (ii) & {$[\%]$} \\
\hline Historic Climate & (a) & $2.531 \mathrm{E}+09$ & $2.676 \mathrm{E}+09$ & $+5.73 \%$ \\
Wet Climate & (b) & $2.891 \mathrm{E}+09$ & $3.118 \mathrm{E}+09$ & $+7.86 \%$ \\
Dry Climate & (c) & $2.278 \mathrm{E}+09$ & $2.487 \mathrm{E}+09$ & $+9.14 \%$ \\
\hline Change (b)-(a) & {$[\%]$} & $+14.21 \%$ & $+16.51 \%$ & \\
Change (c)-(a) & {$[\%]$} & $-9.97 \%$ & $-7.07 \%$ & \\
\hline
\end{tabular}


Table 37: Total groundwater recharge volumes for Middle Thames sub-basin $\left[\mathrm{m}^{3}\right]$

\begin{tabular}{cccccc}
\hline \multicolumn{2}{c}{ Middle Thames (14) } & $\begin{array}{c}\text { Baseline SE } \\
\text { (i) }\end{array}$ & $\begin{array}{c}\text { Infinite SE } \\
\text { (ii) }\end{array}$ & $\begin{array}{c}\text { Change } \\
{\left[\mathrm{m}^{3}\right]}\end{array}$ & $\begin{array}{c}\text { Change } \\
{[\%]}\end{array}$ \\
\hline Historic Climate & (a) & $2.3590 \mathrm{E}+08$ & $2.3444 \mathrm{E}+08$ & $1.4602 \mathrm{E}+06$ & $-0.619 \%$ \\
Wet Climate & (b) & $2.5411 \mathrm{E}+08$ & $2.5295 \mathrm{E}+08$ & $1.1577 \mathrm{E}+06$ & $-0.455 \%$ \\
& & & & & \\
Dry Climate & (c) & $2.3489 \mathrm{E}+08$ & $2.3371 \mathrm{E}+08$ & $1.1810 \mathrm{E}+06$ & $-0.463 \%$ \\
\hline \multicolumn{2}{l}{$\begin{array}{l}\text { Change (b) - (a) } \\
\text { Change (c) - (a) }\end{array}$} & $\begin{array}{l}+3.47 \% \\
-0.86 \%\end{array}$ & $\begin{array}{c}+3.59 \% \\
-0.35 \%\end{array}$ & & \\
\hline
\end{tabular}

Table 38: Total groundwater recharge volumes for North Mitchell sub-basin $\left[\mathrm{m}^{3}\right]$

\begin{tabular}{cccccc}
\hline North Mitchell (20) & $\begin{array}{c}\text { Baseline SE } \\
\text { (i) }\end{array}$ & $\begin{array}{c}\text { Infinite SE } \\
\text { (ii) }\end{array}$ & $\begin{array}{c}\text { Change } \\
{\left[\mathrm{m}^{3}\right]}\end{array}$ & $\begin{array}{c}\text { Change } \\
{[\%]}\end{array}$ \\
\hline Historic Climate & (a) & $2.3592 \mathrm{E}+08$ & $2.3454 \mathrm{E}+08$ & $1.39 \mathrm{E}+06$ & $-0.587 \%$ \\
Wet Climate & (b) & $2.4411 \mathrm{E}+08$ & $2.4295 \mathrm{E}+08$ & $1.16 \mathrm{E}+06$ & $-0.475 \%$ \\
Dry Climate & (c) & $2.2889 \mathrm{E}+08$ & $2.2771 \mathrm{E}+08$ & $1.81 \mathrm{E}+05$ & $-0.515 \%$ \\
\hline \multicolumn{2}{c}{$\begin{array}{c}\text { Change (b) - (a) } \\
\text { Change (c) - (a) }\end{array}$} & $+3.47 \%$ & $+3.59 \%$ & & \\
\hline
\end{tabular}

Table 39: Total groundwater recharge volumes for River Band sub-basin $\left[\mathrm{m}^{3}\right]$

\begin{tabular}{cccccc}
\hline River Bend (27) & $\begin{array}{c}\text { Baseline SE } \\
\text { (i) }\end{array}$ & $\begin{array}{c}\text { Infinite SE } \\
\text { (ii) }\end{array}$ & $\begin{array}{c}\text { Change } \\
{\left[\mathrm{m}^{3}\right]}\end{array}$ & $\begin{array}{c}\text { Change } \\
{[\%]}\end{array}$ \\
\hline Historic Climate & (a) & $5.82 \mathrm{E}+07$ & $5.76 \mathrm{E}+07$ & $5.75 \mathrm{E}+05$ & $-0.988 \%$ \\
Wet Climate & (b) & $6.12 \mathrm{E}+07$ & $6.07 \mathrm{E}+07$ & $4.66 \mathrm{E}+05$ & $-0.742 \%$ \\
& & & & & \\
Dry Climate & (c) & $5.59 \mathrm{E}+07$ & $6.54 \mathrm{E}+07$ & $4.67 \mathrm{E}+05$ & $-0.738 \%$ \\
\hline $\begin{array}{l}\text { Change (b) - (a) } \\
\text { Change (c) - (a) }\end{array}$ & & $+5.08 \%$ & $+5.32 \%$ & & \\
\hline
\end{tabular}




\section{Chapter 6}

\section{Conclusions}

\subsection{Multi-method modeling framework for support of IWRM}

Various global socio-economic and natural processes that have been taking place in the recent decades have placed significant pressures on all natural resources, especially water. Population growth, changes in land use and land cover, and climate variability have substantial effects on human access to water of good quality and quantity, and, therefore, directly influence social, economic and physical well-being of people and ecosystems. Given this sense of urgency, the international water community has advocated the Integrated Water Resources Management (IWRM) paradigm that recognizes the need for coordination in the development and management of water, land and related resources, to maximize economic and social welfare in an equitable manner without compromising ecosystem sustainability, GWP (2000). From the practical standpoint, there is a significant gap between daily water resources engineering practices and the comprehensive definition of IWRM, Biswas (2004). For that reason, Simonovic (2009) suggests the set of principles that should guide all water management activities by IWRM ideals: systems view, integration, partnerships, participation, uncertainty, adaptation and reliance on strong science and reliable data.

Based on the definition and guiding principles, IWRM deals with planning, design and operation of complex systems in order to control the quantity, quality, temporal and spatial distribution of water with the main objective of meeting human and ecological needs and providing protection from water disasters. Since they combine multiple interdependent physical, bio-chemical, social, legal and political processes that drive their performance, water resources systems are among the most complex of human-made and natural systems. To support design, planning and operation, simulation models are used to provide quantitative and qualitative descriptions of water resources systems and replicate their behavior in time and space. The main objective of simulation models is to adequately represent all sources of complexity and to determine its behavior in response 
to different conditions. One of the main challenges of IWRM today is development of modeling tools that operationally implement the concept of integrated water resources management and dynamically couple physical and socio-economic aspects of water resources systems. Literature suggests that only several techniques describe how physical aspects of water resources systems affect and are affected by the social, economic and environmental sub-systems. The vast majority of developed models does not account for interaction between different system components, the complex non-linearity of a system, the feedback mechanisms, and, most importantly, the explicit representation of spatial dimension.

Therefore, this research inspects the role of simulation in IWRM process, analyses the specific advantages and limitations of different modeling methods and, finally, suggests a generic multi-method modeling framework that has as its main goal the capturing of all structural complexities and interactions within water resources systems. Since traditional modeling methods do not provide by themselves adequate support, a multi-method modelling framework is required to properly support implementation of IWRM principles. The presented research adopts the multi-method simulation approach to represent the interconnectedness and the important feedbacks inherent in water resources systems management. The developed methodology is designed to provide support for IWRM through the application of agent-based modeling. The emphasis is placed on explicit modeling and simulation of key aspects of complexity of water resources systems, including:

- $\quad$ Feedback-based system structure;

- Integral representation of physiographic, environmental and socio-economic subsystems and their non-linear interactions;

- $\quad$ Proper consideration of complex spatial and temporal scales of water resources systems;

Explicit representation of the overall system behavior and behavior of system elements as a function of time and space; and 
Provision of support for direct stakeholder participation and involvement.

Consequently, the essential contribution of this research is development of a simulation method that can be used to implement the basic ideas and seven guiding principles of integrated water resources management. First, presented modeling method supports the systems view principle by recognizing a broad set of critical dependencies among water and land resources on one side, and socio-economic environment on the other. Second, it allows for representation of roles of different levels of responsibilities characteristic for water resources systems, both vertical and horizontal. Third, the developed method needs a multidisciplinary approach to deal with complex systems in a holistic way and requires a strong collaboration of the engineering, social, natural, ecological and economic sciences. By representing each individual actor in the system, this modeling method ensures active involvement of all interested stakeholders allowing them individual decision making. Moreover, this method makes the IWRM process more certain by supporting the descriptive assessment of modification of water and land resources caused by numerous human activities. Adaptation principle is supported by building the feedbacks and interactions that take place in water resources system so that adaptive decisions can be made. Finally, the presented method demands the involvement of numerous scientific domains that are important from the aspect of IWRM (hydrology, hydraulics, geology, meteorology, etc.)

A new multi-method modeling framework presented in this thesis has a potential to expand modeling capabilities in numerous areas where spatial and temporal processes are of the main interest.

Based on the architecture of the generic modeling framework, the operational Integrated Hydrologic-Socio-Economic Model for the Upper Thames River Basin (IHSEM-UTRB) is designed to facilitate implementation of the main ideas of IWRM in a local river basin. The developed model is sophisticated considering that it includes the spatially explicit feedbacks between hydrologic and socio-economic system components and the fact that the integration of all system components is performed under a common user-friendly graphical interface. This directly allows the model user to change the model structure, 
test policies and perform numerous simulations. A new multi-method modeling framework is implemented by using the agent-based modeling approach to represent regional socio-economic environment, and this component is coupled via a feedback link with a continuous hydrologic model that describes the underlying physical processes. The presented model, developed using open source programming platforms, allows for testing of a wide range of policies and management strategies. Also, model produces detailed hydrologic response to human induced changes in the basin at different spatial and temporal scales. The model analyses two types of changes: changes in land use and changes in use of water. Hydrologic regimes in the UTRB are studied using a set of three climate inputs with a spatially semi-distributed continuous hydrologic model, while socio-economic impacts are analyzed using a spatially explicit agent-based socioeconomic model. Six different combinations scenarios are used to explore the effects of climate change variations and socio-economic conditions on overall system behavior. After simulating combinations of different scenarios and assessing their impacts the obtained results show co-dependence between changes in hydrologic regime and regional socio-economic activities. These conclusions demonstrate ways in which different climate conditions, coupled with policies and management strategies, have the potential to alter the physical and socio-economic landscape of the UTRB. Socio-economic environment can potentially be altered as a result of both changes in climatic conditions and in management practices. Results show that the water availability is a cornerstone of future social and economic development. With increasing population and expansion of industrial and agricultural activities, water use is also expected to increase. Increased development and urbanization change local hydrologic regimes by converting lands with high recharge rates (agricultural and forested lands) into less resilient residential, industrial or commercial lands.

\subsection{Opportunities for future work}

The IWRM is a holistic approach that must be adapted to different local and regional contexts. This paradigm cannot be blindly applied to any arbitrary social or environmental context since water problems differ significantly from one region to another. Therefore, the methodology presented here is designed to support the main 
principles of integrated water resources management, but in such a way that it can be applied to other contexts and potential problems. However, different contexts are likely to have different characteristics of climate, hydrologic and socio-economic systems, and, therefore, all individual components must be properly revised and adjusted accordingly.

Presented operational model, developed on the basis of presented methodology, relies on the previous studies of projections of future climate conditions in the UTRB. Therefore, the developed methodology can be improved by adding the climate modeling component which would allow much more extensive investigation of future conditions. This component would analyze the broader range of future climate conditions as projected by the latest Global Climate Models (GCMs) and Earth System Models (ESMs) as outlined in the Fifth Assessment Report (AR5) of the Intergovernmental Panel for Climate Change (IPCC, 2014). All twenty-four models and four Representative Concentration Pathways (RCPs) can be considered to capture the entire range of climatic changes projected for future. Also, recently future land-cover projections have been made by four different climate modelling groups. These future projections correspond to different future emission scenarios (Hurtt et al. 2011). Land-cover projections made by these modelling groups can be used to quantify their local impacts on future flow projections.

For each context, the central hydrologic component must be developed according to the local conditions, and then it must be properly calibrated and verified. The identical structure of the hydrologic model can be applied to other regions, but different model parameters must be selected to reflect local climatic and physiographic conditions. One of the potential directions for future improvements of the system structure could be introduction of spatially fully distributed hydrologic models. These distributed physically-based watershed models integrate processes such as ground and surface water dynamics, and recharge and evapotranspiration rates. Integrated flow models couple surface and subsurface flow systems with groundwater recharge and discharge. This can help analysis of impacts of groundwater and surface water withdrawals. The model can evaluate the effects of increased water pumping for municipal supply on local streamflow. Spatially distributed models are typically used for the analysis, planning and management of problems related to surface-water impact from groundwater withdrawal, 
conjunctive use of groundwater and surface water, wetland management and restoration, river basin management and planning, and impact studies for changes in land use and climate.

One of the important assumptions made in the presented work is that the rates of water withdrawals are calculated using the maximum pumping rate and frequency over a period of time provided by the PTTW database. However, this assumption gives higher values than what is normally pumped. Also, estimates provided by PTTW database do not consider the consumptive nature of water takings since the permit holders are required to report only the total pumping volumes, not the volume and the location of returned water. To obtain the consumptive water demand, the reported rates typically must be modified by a consumptive use factor.

Different regions also have different socio-economic properties characterized by different economic drivers, urbanization and development processes, water supply and use practices, cultural values, environmental sensitivities, etc. Therefore, additional research should be undertaken to study ongoing feedback links within the model and thus improve the model structure. This can be done by engaging social and economic studies that explore the relationships between different model variables. Partnership with other scientific domains could reveal a number of relationships that are coarsely assumed in this research and on which the current socio-economic model is built. For example, the developed model assumes that there is no direct dependence between water quality and socio-economic activities in this region. However, the potential effects of changes induced by socio-economic activities in a basin are changes in quality of surface and groundwater, too. In this case, the water quality characteristics are not considered, but this component, designed on appropriate spatial and temporal scales, could be added to the system structure to further enhance the structural validity of the model.

The agent-based model developed in this research is primarily designed to represent the spatial variability of water resources systems. But the true challenge of agent-based modeling is learning how to model agent behaviors in ways to represent realistic system behavior. Numerous scientific domains offer many ways to model behavior of different 
kinds of agents (people, animals, vehicles, organizations, etc.) and one of the main tasks in water resources management is to discover the dominant drivers of behavior related to use of natural resources. According to Railsback and Grimm (2011) human behavior and decision-making are subjects of entire fields of study. For example, the new field of behavioral economics and behavioral finance studies suggests how people actually make the decisions that can be represented in agent-based models. The "simple heuristics" approach suggests that people make complex decisions in uncertain contexts via very simple rules. On the other hand, the evolutionary approach uses agent-based models to "evolve" mathematical properties that cause agents to reproduce observed patterns of individual and system level behavior. In this case, behavior of an agent is represented using neural networks. These tend to link decision outcomes to one or more sensory inputs. However, despite the fact that agent-based models focus mainly on developing theories for agent behavior, they are still only system models. This directly implies that agents should be as complex as necessary to capture the critical effects of behavior on the system.

One of the advantages offered by a bottom-up modeling approach, such as agent-based simulation, is its ability to directly control the behavior of individual elements, in this case agents, and observe the direct consequences on system performance. The Netlogo agent-based modeling environment contains the HubNet protocol that can be used to create direct links between the model participants and agents represented in the model. In this way, all recognized system stakeholders can actively participate in the simulation process, and guide the behavior of their respective agents. This approach enables them to directly see the consequences of their individual actions and resulting system behavior. 


\section{References}

Ahmad, S., and Simonovic, S.P. (2000a). "Analysis of economic and social impacts of flood management policies using system dynamics." Proc. Int. Conf. of the American Institute of Hydrology. Atmospheric, Surface and Subsurface Hydrology and Interactions, Research Triangle Park, N.C.

Ahmad, S., and Simonovic, S.P. (2000b). "Dynamic modeling of flood management policies." Proc. 18th Int. Conf. of the System Dynamics Society, Sustainability in the Third Millennium, Bergen, Norway

Ahmad, S., and Simonovic, S.P. (2000c). "System dynamics modeling of reservoir operation for flood management." J Comput Civ Eng 14(3):190-198

Ahmad, S. (2002). An intelligent decision support system for flood management: a spatial system dynamics approach. Doctoral Dissertation, Department of Civil and Environmental Engineering, The University of Western Ontario, London, Canada

Akhbari, M., and Grigg, N. S. (2013). “A Framework for an Agent-Based Model to Manage Water Resources Conflicts.” Water Resources Management, 27, 4039-4052. doi:10.1007/s11269-013-0394-0

Anselin, L. (2002). "Under the hood: Issues in the specification and interpretation spatial regression models." Agricultural economics, 27 (3): 247-267

AnyLogic (2015). AnyLogic homepage. Available at http://www.xjtek.com/anylogic/ (accessed January 2015)

Arnell, V. (2002) Hydrology and Global Environmental Change. Prentice Hall, ISBN 0582369843

Barrow, E., Maxwell, B. and Gachon, P. (2004). Climate Variability and Change in Canada: Past, Present and Future, ACSD Science Assessment Series No. 2, Meteorological Service of Canada, Environment Canada, Toronto, Ontario 
Becu, N., Perez, P., Walker, A., Barreteau, O., and Page, C.L. (2003). “Agent based simulation of a small catchment water management in northern Thailand." Ecological Modelling, 170(2-3), 319-331. doi:10.1016/S0304-3800(03)00236-9

BenDor, T. and Kaza, N. (2011). "Theory of Spatial Reference Modes and System Archetypes." System Dynamics Winter Conference in January 2011, Austin Texas

BenDor, T. and Metcalf, S. (2006). "The spatial dynamics of invasive species spread." System Dynamics Review 22(1): 27-50.

Bicknell, B.R., Imhoff, J.C., Kittle, J.L., Jobes, T.H., and Donigian, A.S. (2001). Hydrological Simulation Program-FORTRAN (HSPF), User's manual for version 12. Aqua Terra Consultants, Mountain View, California.

Birkinshaw, S.J., James, P. and Ewen, J. (2010). "Graphical User Interface for Rapid Set- up of SHETRAN Physically-Based River Catchment Model." Environmental Modelling \& Software, 25, 609-610.

Biswas, A.K. (2004). Integrated Water Resources Management: A Reassessment. Water International, 29(2), 248-256. doi:10.1080/02508060408691775

Brooks, G.R., Evans S.G. and Clague J.J. (2001). Flooding. A Synthesis of Natural Geological Hazards in Canada. G.R. Brooks (ed.). Ottawa: Geological Survey of Canada Bulletin 548.101-143

Brutsaert, W. (1982). Evaporation into the Atmosphere: theory, history and applications. Kluwer Academic Publishers, Dordrecht, Netherlands.

Cai, X., McKinney, D. C., and Lasdon, L.S. (2003). "Integrated hydrologic-agronomiceconomic model for river basin management”. ASCE J Water Resour. Plan Manage 129(1):4-17

Cai, X., Yang, Y. E., and Ringler, C. (2011). "Can Water Allocation in the Yellow River Basin Be Improved? Insights from a Multi-Agent System Model.” IFPRI Discussion Papers, 2011 
Clavel, L., Charron, M.H., Therond, O., and Leenhardt, D. (2012). “A Modelling Solution for Developing and Evaluating Agricultural Land-Use Scenarios in Water Scarcity Contexts." Water Resources Management, 26(9), 2625-2641. doi:10.1007/s11269012-0037-X

Coelho, A.C., Labadie, J.W., and Fontane, D.G. (2012). "Multicriteria Decision Support System for Regionalization of Integrated Water Resources Management." Water Resources Management, 26(5), 1325-1346. doi:10.1007/s11269-011-9961-4

Constance, E.H. (2004). Thirsty Planet: Strategies for Sustainable Water Management. Zed Books, New York, New York

Conservation Ontario (2014). Conservation Ontario homepage. Available at: http://www.conservation-ontario.on.ca/ (accessed on September 2014)

Cosgrove, C.E. and Cosgrove, W.J. (2011). The Dynamics of Global Water Futures Driving Forces 2011 - 2050. Report on the findings of Phase One of the UNESCOWWAP Water Scenarios Project to 2050. United Nations World Assessment Programme

Cunderlik, J.M. and Simonovic, S.P. (2003). “Assessment of water resources risk and vulnerability to changing climatic conditions: Hydrologic model selection for the CFCAS project.” Report No. I, Department of Civil and Environmental Engineering, The University of Western Ontario, London, Ontario, Canada.

Cunderlik, J.M. and Simonovic, S.P. (2004). “Assessment of water resources risk and vulnerability to changing climatic conditions: Calibration and verification data for the HEC-HMS hydrologic model.” Report No. II, Department of Civil and Environmental Engineering, The University of Western Ontario, London, Ontario, Canada.

Cunderlik, J.M. and Simonovic, S.P. (2005). "Hydrological extremes in a southwestern Ontario river basin under future climate conditions.” IAHS Hydrological Sciences Journal, 50(4), 631-654. 
Dawadi, S., and Ahmad, S. (2012). "Changing Climatic Conditions in the Colorado River Basin: Implications for Water Resources Management." Journal of Hydrology, 430431 127-141.

Déry, S.J. (2005). "Decreasing river discharge in northern Canada." Geophysical Research Letters, 32(10), L10401. doi:10.1029/2005GL022845

DHI (2004). MIKE SHE User Manual. Hørsholm, Denmark: Danish Hydraulic Institute.

Dragicevic, S. (2010). “Cellular Automata." Encyclopedia of Geography, edited by Barney Warf, 369-72. Thousand Oaks, CA: SAGE Publications, Inc., 2010. doi: http://dx.doi.org/10.4135/9781412939591.n141

EM-DAT (2014). The International Disaster Database. http://www.emdat.be/disastertrends (accessed December 2014)

Environment Canada (2004). Threats to Water Availability in Canada. National Water Research Institute, Burlington, Ontario. NWRI Scientific Assessment Report Series No. 3 and ACSD Science Assessment Series No. 1.

ESRI (2014). ArcGis Desktop. http://help.arcgis.com (accessed December 2014)

Filatova, T., Parker, D., and van der Veen, A. (2009). "Agent-based urban land markets: Agent's pricing behavior, land prices and urban land use change."Journal of Artificial Societies and Social Simulation, 12 (1). http://jasss.soc.surrey.ac.uk/12/1/3.html

Fletcher, E.J. (1998). "The use of system dynamics as a decision support tool for the management of surface water resources." Proc. 1st Int. Conf. on New Information Technologies for Decision Making in Civil Engineering, Montreal, 909-920

Ford, A. (1996). "Testing the Snake river explorer." Syst Dyn Rev 12(4):305-329, Winter

Ford, A. (1999). "Modeling the Environment: An Introduction to System Dynamics Modeling of Environmental Systems.” Island Press: Washington, DC. 
Forrester, J.W. (1961). Industrial dynamics. The MIT Press, Cambridge, Massachusetts

Forrester, J.W. (1991). From the ranch to system dynamics: An autobiography. In A.G. Bedeian (Ed.). Management laureates: A collection of autobiography essays (Vol. 1). Connecticut: JAI Press.

Ghimire, B., Chen, A.S., Guidolin, M., Keedwell, E.C., Djordjević, S., and Savić, D. (2013). "Formulation of a fast $2 D$ urban pluvial flood model using a cellular automata approach." Journal of Hydroinformatics, 15, 676. doi:10.2166/hydro.2012.245

Greenland International Consulting Inc., (2001). Visual OTTHYMO User's Guide, version 2.0. Concord, Ontario, Canada.

Grimm, V. and Railsback, S.F. (2005). Individual-based modeling and ecology. Princeton: Princeton University Press, ISBN: 0691096651

GWP (2000). Integrated water resources management (TAC Background Paper No. 4). Global Water Partnership. Stockholm: Technical Advisory Committee, Global Water Partnership.

Hassanzadeh, E., Elshorbagy, A., Wheater, H., and Gober, P. (2014). "Managing water in complex systems: An integrated water resources model for Saskatchewan, Canada." Environmental Modelling \& Software, 58, 12-26. doi:10.1016/j.envsoft.2014.03.015

Hassing, J., Ipsen, N. and Clausen, T.J. (2009). Integrated Water Resources Management in Action. The United Nations World Water Assessment Programme. Dialogue Paper.

Heppenstall, A.J. et al. (2012). Agent-Based Models of Geographical Systems, 253 doi: 10.1007/978-90-481-8927-4_13, Springer Science Business Media B.V. 2012

Hurtt, G.C., et al. (2011). Harmonization of land-use scenarios for the period 15002100: 600 years of global gridded annual land-use transitions, wood harvest, and resulting secondary lands. Clim. Change, 109(1-2): 117-161. 
IISD (2009). "A Guide to Creating Adaptive Policies - Seven things policy-makers should know to craft better policies in today's dynamic and uncertain world." Eds. D. Swanson and S. Bhadwal.

IPCC (2001). Climate Change 2001: Scientific Basis. Contribution of the Working Group I to the Third Assessment Report of the Intergovernmental Panel on Climate Change. Cambridge University Press, Cambridge, United Kingdom.

IPCC (2013). Climate Change 2013: The Physical Science Basis. Contribution of Working Group I to the Fifth Assessment Report of the Intergovernmental Panel on Climate Change [Stocker, T.F., D. Qin, G.-K. Plattner, M. Tignor, S.K. Allen, J. Boschung, A. Nauels, Y. Xia, V. Bex and P.M. Midgley (eds.)]. Cambridge University Press, Cambridge, United Kingdom and New York, NY, USA, 1535 pp, doi:10.1017/CBO9781107415324.

IPCC (2014). Summary for Policymakers. In: Climate Change 2014: Impacts, Adaptation, and Vulnerability. Part A: Global and Sectoral Aspects. Contribution of Working Group II to the Fifth Assessment Report of the Intergovernmental Panel on Climate Change [Field, C.B., V.R. Barros, D.J. Dokken, K.J. Mach, M.D. Mastrandrea, T.E. Bilir, M. Chatterjee, K.L. Ebi, Y.O. Estrada, R.C. Genova, B. Girma, E.S. Kissel, A.N. Levy, S. MacCracken, P.R. Mastrandrea, and L.L. White (eds.)]. Cambridge University Press, Cambridge, United Kingdom and New York, NY, USA, pp. 1-32.

Karamouz, M. Zahraie, B. and Szidarovszky, F. (2003). Water resources systems analysis. Lewis Publishers, USA. ISBN 1-56670-642-4

Kennedy, W.G. (2012). Modelling Human Behaviour in Agent-Based Models. AgentBased Models of Geographical Systems. (pp. 167-179). doi:10.1007/978-90-481$8927-4$

Keyes, A.M., and Palmer, R. (1993). "The role of object oriented simulation models in the drought preparedness studies." Proc. Water Management in 90's: a time for innovation, ASCE, New York 
Lättilä, L., Hilletofth, P., and Lin, B. (2010). "Hybrid simulation models - When, Why, How?" Expert Systems with Applications, 37(12), 7969-7975. doi:10.1016/j.eswa.2010.04.039

Lemmen, D.S.,Warren, F.J., Lacroix, J., and Bush, E.(2008). From Impacts to Adaptation: Canada in a Changing Climate 2007; Government of Canada, Ottawa, $\mathrm{ON}, 448 \mathrm{p}$

Ligmann-Zielinska, A. (2010). "Agent-Based Models.” Encyclopedia of Geography, edited by Barney Warf, 28-32. Thousand Oaks, CA: SAGE Publications, Inc. doi: http://dx.doi.org/10.4135/9781412939591.n14

Linsley, R.K., Kohler, M.A. and Paulhus, J.L.H. (1958). Hydrology for Engineers. McGraw-Hill Book Company, Inc., New York.

Loucks, D.P. and van Beek, E. (2005). Water Resources Systems Planning and Management: An Introduction to Methods, Models and Applications. United Nations Educational, Scientific and Cultural Organization, Paris, France and Delft, Netherlands.

Luke, S., Cioffi -Revilla, C., Panait, L., and Sullivan, K. (2004). MASON: A new multiagent simulation toolkit. SwarmFest 2004, Eighth Annual Swarm Users/Researchers Conference, University of Michigan, Ann Arbor

Mainuddin, M., Kirby, M., and Qureshi, M.E. (2007). “Integrated hydrologic-economic modelling for analyzing water acquisition strategies in the Murray River Basin." Agricultural water management vol. 93, 123 - 135

Martinez-Santos P., Aldaya M.M., Llamas M.R., Laurens A.D. and Thuy R. (2014). Integrated Water Resources Management in the 21st Century: Revisiting the paradigm, ISBN: 978-1-138-00143-5, CRC Press

Meadows, D. (2008). Thinking in Systems: A Primer. ISBN-13: 978-1603580557Chelsea Green Publishing 
Mekonnen, M.M. and Hoekstra, A.Y. (2011a). The green, blue and grey water footprint of crops and derived crop products. Hydrology and Earth System Sciences, 15(5): 1577-1600.

Mekonnen, M.M. and Hoekstra, A.Y. (2011b). National water footprint accounts: the green, blue and grey water footprint of production and consumption. Value of Water Research Report Series No.50, UNESCO-IHE, Delft, the Netherlands.

Meyers, R.A. (2009). Encyclopedia of Complexity and Systems Science. ISBN 978-0387-69572-3 Springer-Verlag New York

Mitchell, B., Priddle, C., Shrubsole, D., Veale, B., and Walters, D. (2014). "Integrated water resource management: lessons from conservation authorities in Ontario, Canada." International Journal of Water Resources Development, 30(3), 460-474. doi:10.1080/07900627.2013.876328

MMAH (2002). London Regional Analysis. Planning Policy Section, Provincial Planning and Environmental Services Branch, Ministry of Municipal Affairs and Housing, London, Ontario, Canada

Morin, A. and Cantin, B. (2009). Strengthening Integrated Water Resource Management in Canada. ISBN number: PH4-54/2009E-PDF 978-1-100-12291-5

Neuwirth, C., Peck, A. and Simonovic, S.P. (2015). “Modeling structural change in spatial system dynamics: A Daisyworld example." Environmental Modelling \& Software, 65, 30-40. doi:10.1016/j.envsoft.2014.11.026

Nirupama, N. and Simonovic S.P. (2002). Role of remote sensing in disaster management. Water Resources Research Report no. 040, Facility for Intelligent Decision Support, Department of Civil and Environmental Engineering, London, Ontario, Canada, 107 pages. ISBN: (print) 978-0-7714-2610-0; (online) 978-0-77142611-7.

North, M.J. and Macal, C.M. (2009). "Agent-based modeling and systems dynamics model replication. " International Journal of Simulation and Process Modelling, 2009 
North, M.J., Collier, N.T., Ozik, J., Tatara, E., Altaweel, M., Macal, C.M., Bragen, M., and Sydelko, P. (2013). "Complex Adaptive Systems Modeling with Repast Simphony." Complex Adaptive Systems Modeling, Springer, Heidelberg, FRG http://www.casmodeling.com/content/1/1/3

Ontario (2014). Ontario Permit To Take Water Database http://www.ontario.ca/data/permit-take-water (accessed December 2014)

OpenStarLogo (2015). OpenStarLogo homepage. Available at http://education.mit.edu/openstarlogo/ (accessed December 2014)

Ota S. (2009). IWRM guidelines at river basin level, UNESCO

Prodanovic, P. (2007). Response of water resources systems to climate change. Doctoral Thesis, Department of Civil and Environmental Engineering, The University of Western Ontario, London, Canada

Prodanovic, P. and Simonovic, S.P. (2010). “An operational model for integrated water resources management of a watershed.” Int J Water Resour Manag 24(6):1161-1194

Railsback, S.F. and Grimm, V. (2011). Agent-based and Individual-Based Modeling: A Practical Introduction. Princeton: Princeton University Press, ISBN: 978-0-691$13674-5$

Raymond J.K., Loucks D.P., and Stedinger J.R. (2012). “Artificial neural network models of watershed nutrient loading." Int J Water Resour Manag 26:2781-2797. doi:10.1007/s11269-012-0045-X

Roach, J. and Tidwell, V. (2009). “A Compartmental-Spatial System Dynamics Approach to Ground Water Modeling." Ground Water Volume: 47, Issue: 5, Pages: 686-698

Rossman, L.A. (2004). Storm Water Management Model (SWMM) User's Manual, version 5.0. United States Environmental Protection Agency, Cincinnati, Ohio. 
Saqalli, M., Thiriot, S., D, F. A., Capitole, T., and Gabriel, D. (2010). "Investigating Social Conflicts Linked to Water Resources through Agent-Based Modelling". NATO Science for Peace and Security Series 75 (2010) 142-157

Schieritz, N. and Milling, P. (2003). Modeling the forest or modeling the trees. Proceedings of the 21st International System Dynamics Society, (Phelan 1999), 115.

Scholargeoportal (2014). Scholar Geo Portal http://geo2.scholarsportal.info/\#r/tab/browseTab (accessed December 2014)

Sharif, M. and Burn, D. H. (2004). “Assessment of water resources risk and vulnerability to changing climatic conditions: Development and application of a $K$-NN weather generating model." Report No. III, Department of Civil and Environmental Engineering, The University of Western Ontario, London, Ontario, Canada.

Sharif, M. and Burn, D. H. (2006). "Simulating climate change scenarios using an improved K-Nearest Neighbor model.” Journal of Hydrology, 325, 179-196.

Sharif, M. and Burn, D. H. (2007). "Improved K-Nearest Neighbor weather generating model.” ASCE Journal of Hydrologic Engineering, 12(1), 42-51.

Shiklomanov, I.A. (2000). “Appraisal and Assessment of World Water Resources.” Water International, 25(1), 11-32. doi:10.1080/02508060008686794

Shrubsole, D. et al. (2003). "An assessment of flood risk management in Canada." ICLR Research Paper Series - No. 28. ISBN 0-9732213-6-4

Simonovic, S.P. (2009a), “Water Resources Management: A Systems View”, Water Front, invited article, 1:12-13.

Simonovic, S.P. (2009b). Managing water resources: methods and tools for a systems approach. UNESCO, Paris and Earthscan James \& James, London, ISBN 978-184407-554-6 
Simonovic, S.P. and Li, L. (2003). "Methodology for assessment of climate change impacts on large-scale flood protection system." J Water Resour Plan Manag 129(5):361-371

Sivapalan, M., Savenije, H.H.G., and Blöschl, G. (2012). "Socio-hydrology: A new science of people and water." Hydrological Processes, 26(8), 1270-1276. doi:10.1002/hyp.8426

Statistics Canada (2010a). Human Activity and the Environment: Freshwater supply and Demand Canada. Ottawa, Ontario, Statistics Canada

Statistics Canada (2010b). Agricultural Water Use in Canada. Statistics Canada, Environment Accounts and Statistics Division, Ottawa, Ontario, Canada.

Statistics Canada (2011). Census: Community Profiles. Statistics Canada, Ottawa, Ontario, Statistics Canada.

Stave, K.A. (2003). "A system dynamics model to facilitate public understanding of water management options in Las Vegas, Nevada. ”J Environ Manag 67:303-313

Sterman, J.D. (2000). Business Dynamics: Systems Thinking and Modeling for a Complex World. Irwin McGraw-Hill, Boston, Massachusetts.

Swarm (2014). Swarm: A platform for agent-based models. Available at http://www.swarm.org/ (Accessed on December 2014)

Thames - Sydenham and Region Drinking Water Source Protection (2014). http://www.sourcewaterprotection.on.ca/ (accessed December 2014)

Tidwell, V.C., Passell, H.D., Conrad, S.H., and Thomas, R.P. (2004). "System dynamics modeling for community-based water planning: application to the Middle Rio Grande." Aquat Sci 66:357-372. doi:10.1007/s00027-004-0722-9

UN (1992). Protection of the Quality and Supply of Freshwater Resources: Application of Integrated Approaches to the Development, Management and Use of Water 
Resources. United Nations Conference on Environment and Development, Rio de Janeiro, 1992, "Earth Summit" Agenda 21, Chapter 18.

UNEP (2012). The UN-Water Status Report on the Application of Integrated Approaches to Water Resources Management.

USACE (2000). Hydrologic Modelling System HEC-HMS, Technical reference manual. United States Army Corps of Engineers, Hydrologic Engineering Center, Davis, California.

USACE (2006). Hydrologic Modelling System HEC-HMS, User's Manual for version 3.0.1. United States Army Corps of Engineers, Hydrologic Engineering Center, Davis, California.

UTRCA (2012). The Upper Thames River Watershed: Report Cards 2012. Upper Thames River Conservation Authority, London, Ontario, Canada.

Valkering, P., and Offermans, A. (2009). "Modeling Cultural and Behavioral Change in Water Management: An Integrated, agent based, gaming approach." The Integrated Assessment Journal, Vol. 9, Iss. 1, Pp. 19-46.

van Oel, P. (2009). Water-scarcity patterns, spatiotemporal interdependencies between water use and water availability in a semi-arid river basin. Doctoral thesis, University of Twente, Twente, the Netherlands

Vidal, J.M. (2010). Fundamentals of Multiagent Systems with Netlogo Examples.

Vionov, A., Constanza, R., Fitz, C. and Maxwell, T. (2007). "Patuxent landscape model: Hydrological model development." Water resources research, 34 (2):163-170

Ward, F.A., Booker, J. F., and Michelsen, A.M. (2006). "Integrated Economic, Hydrologic, and Institutional Analysis of Policy Responses to Mitigate Drought Impacts in Rio Grande Basin." J Water Resour Plan Manag, Vol. 132, No. 6, November 1, 488-502. 
Wey, K. (2006). Temporal disaggregation of daily precipitation data in a changing climate. Masters Thesis, Department of Civil Engineering, University of Waterloo, Waterloo, Ontario, Canada.

WHO (2014). Progress on Drinking Water and Sanitation. World Health Organization and UNICEF Joint Monitoring Programme (JMP).

Wilcox, I., Quinlan, C., Rogers, C., Troughton, M., McCallum, I., Quenneville, A., Heagy, E., and Dool, D. (1998). The Thames River Watershed: A Background Study for Nomination under the Canadian Heritage Rivers System. Upper Thames River Conservation Authority, London, Ontario, Canada.

Wilensky, U. (1999). NetLogo. Center for Connected Learning and Computer-Based Modeling, Northwestern University, Evanston, IL http://ccl.northwestern.edu/netlogo/ (accessed December 2014)

Winz, I., Brierley, G., and Trowsdale, S. (2008). "The Use of System Dynamics Simulation in Water Resources Management." Water Resources Management, 23(7), 1301-1323. doi:10.1007/s11269-008-9328-7

Wooldridge, M. (2009). An Introduction to MultiAgent Systems. John Wiley \& Sons Ltd, 2002, ISBN 0-471-49691-X.

World Bank (2013). World Development Report 2014: Risk and Opportunity_Managing Risk for Development. Washington, DC: World Bank. doi: 10.1596/978-0-8213-99033. License: Creative Commons Attribution CC BY 3.0

Wurbs, R.A. (1994). Computer Models for Water Resources Planning and Management. USACE, Alexandria, Virginia

WWAP (2012). Managing Water under Uncertainty and Risk. The United Nations World Water Development Report 4. World Water Assessment Programme. Paris, UNESCO

Yang, Y.C.E., Cai, X., and Stipanović, D.M. (2009). “A Decentralized Optimization Algorithm for Multi-Agent System Based Watershed Management." World 
Environmental and Water Resources Congress 2009, (1), 1-8. doi:10.1061/41036(342)463

Yu, H. (2010). “Temporal GIS.” Encyclopedia of Geography, edited by Barney Warf, 2797-98. Thousand Oaks, CA: SAGE Publications, Inc., 2010. doi: http://dx.doi.org/10.4135/9781412939591.n1124.

Zechman, E.M. (2011). "Agent-based modeling to simulate contamination events and evaluate threat management strategies in water distribution systems." Risk Analysis : An Official Publication of the Society for Risk Analysis, 31(5), 758-72. doi:10.1111/j.1539-6924.2010.01564.x 


\section{Appendices}

\section{Appendix A}

\section{Spatial database - list of attributes}

This contains lists of attributes for all shapefiles detailed in Section 4.2.1.

UTSubbasins.shp

| $\underline{\text { FID }}$ | Shape* | OBJECTID | OBJECT_ID | DESCR | GUT_NUMBER | IDENT | NAME | VERISTT_FL |

\begin{tabular}{|c|c|c|c|c|c|c|c|} 
VERIST & BUSEFFDT & BUS_EFF_ & ACCURA & SENS_CLA & SENS_DA & SENS_R & SENS_DES \\
DT & FL & DT & CY & SS & TE & AT & CR
\end{tabular}

\begin{tabular}{|c|c|c|c|c|c|c|c|} 
SYS_ARE & SYS_LENG & USER_CA & GNL_C & EFF_DA & SHAPE_LE & SHAPE_AR & BASIN_AR \\
A & TH & LC & MT & TE & NG & EA & EA
\end{tabular} | SB_AREA | MNCPLTY | AREA_PERC | PRIVATEUSE | AGRIUSE |

UTUrbanCenters.shp

| FID | Shape* | NAME | PROV | LONGITUdE | LATITUDE | PPN_CODE | PREC_CODE | MJR_CITY | | PRCDCSD | CSD_NAME | CSD_POP00 | PPN_ID | RETIRED | CITY_ID |

UTHydrography.shp

| FID $\mid$ Shape* $\mid$ OBJECTID | HYNAME | SHAPE_LENG |

UTGaugeStations_2014.shp

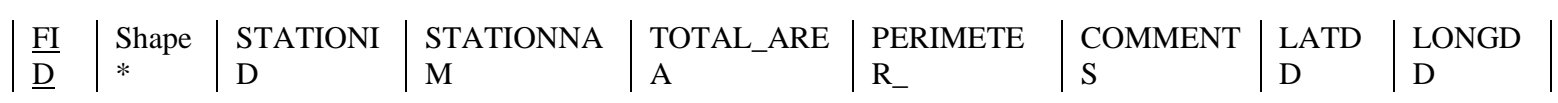
| DATE_GENER | WSHEDCOMPL |

UTCounties.shp

\begin{tabular}{|c|c|c|c|c|c|c|c|c|c|} 
FI & Shape & OBJECTI & CDUI & CDNAM & CDTYP & PRUI & PRNAM & SHAPE_LEN & SHAPE_ARE \\
$\underline{D}$ & $*$ & D & D & E & E & D & E & G & A
\end{tabular}


UTAgriculturalPPTW_2014.shp

\begin{tabular}{|c|c|c|c|c|c|c|c|c|}
$\underline{\mathrm{FI}}$ & Shape & OBJECTI & PERMITN & CLIENTNA & PURPOSEC & SPURPO & EXPIRYDA & ISSUEDDA \\
$\underline{\mathrm{D}}$ & $*$ & $\mathrm{D}$ & $\mathrm{O}$ & $\mathrm{ME}$ & $\mathrm{AT}$ & $\mathrm{SE}$ & TE & TE
\end{tabular} \begin{tabular}{|c|c|c|c|c|c|c|c|c|} 
RENEWDA & OLDCTYT & P_LO & P_CO & P_MUNIC & P_UPPE & P_LOWE & SURFGRN & SOURCEI \\
TE & WN & T & N & IP & RT & RT & D & D
\end{tabular} \begin{tabular}{|c|c|c|c|c|c|c|c|} 
EASTIN & NORTHIN & UTMZON & MAXL_DA & DAYS_YE & HRS_DAYM & L_MINUT & AMENDED_ \\
G & G & E & Y & AR & AX & E & BY
\end{tabular} | EXPIRED_BY | PERMIT_END | ACTIVE | LATITUDE | LONGITUDE | LINK_TO_SW | LINK_TO_MN | | ISSUE_TICK | EXPIRY_TIC |

UTCommercialPPTW_2014.shp

\begin{tabular}{|c|c|c|c|c|c|c|c|c|}
$\underline{\mathrm{FI}}$ & Shape & OBJECTI & PERMITN & CLIENTNA & PURPOSEC & SPURPO & EXPIRYDA & ISSUEDDA \\
$\underline{\mathrm{D}}$ & $*$ & $\mathrm{D}$ & $\mathrm{O}$ & $\mathrm{ME}$ & AT & SE & TE & TE
\end{tabular}
\begin{tabular}{|c|c|c|c|c|c|c|c|c} 
RENEWDA & OLDCTYT & P_LO & P_CO & P_MUNIC & P_UPPE & P_LOWE & SURFGRN & SOURCEI \\
TE & WN & T & N & IP & RT & RT & D & D
\end{tabular} \begin{tabular}{|c|c|c|c|c|c|c|c|} 
EASTIN & NORTHIN & UTMZON & MAXL_DA & DAYS_YE & HRS_DAYM & L_MINUT & AMENDED_ \\
G & G & E & Y & AR & AX & E & BY
\end{tabular} | EXPIRED_BY | PERMIT_END | ACTIVE | LATITUDE | LONGITUDE | LINK_TO_SW | LINK_TO_MN | | ISSUE_TICK | EXPIRY_TIC |

UTIndustrialPPTW_2014.shp

\begin{tabular}{|c|c|c|c|c|c|c|c|c|}
$\underline{\underline{F I}}$ & Shape & OBJECTI & PERMITN & CLIENTNA & PURPOSEC & SPURPO & EXPIRYDA & ISSUEDDA \\
$\underline{\mathrm{D}}$ & $*$ & $\mathrm{D}$ & $\mathrm{O}$ & $\mathrm{ME}$ & AT & SE & TE & TE
\end{tabular} \begin{tabular}{|c|c|c|c|c|c|c|c|c|} 
RENEWDA & OLDCTYT & P_LO & P_CO & P_MUNIC & P_UPPE & P_LOWE & SURFGRN & SOURCEI \\
TE & WN & T & N & IP & RT & RT & D & D
\end{tabular} \begin{tabular}{|c|c|c|c|c|c|c|c|} 
EASTIN & NORTHIN & UTMZON & MAXL_DA & DAYS_YE & HRS_DAYM & L_MINUT & AMENDED_ \\
G & G & E & Y & AR & AX & E & BY
\end{tabular} | EXPIRED_BY | PERMIT_END | ACTIVE | LATITUDE | LONGITUDE | LINK_TO_SW | LINK_TO_MN |

| ISSUE_TICK | EXPIRY_TIC | 
UTWaterSupplyPPTW_2014.shp

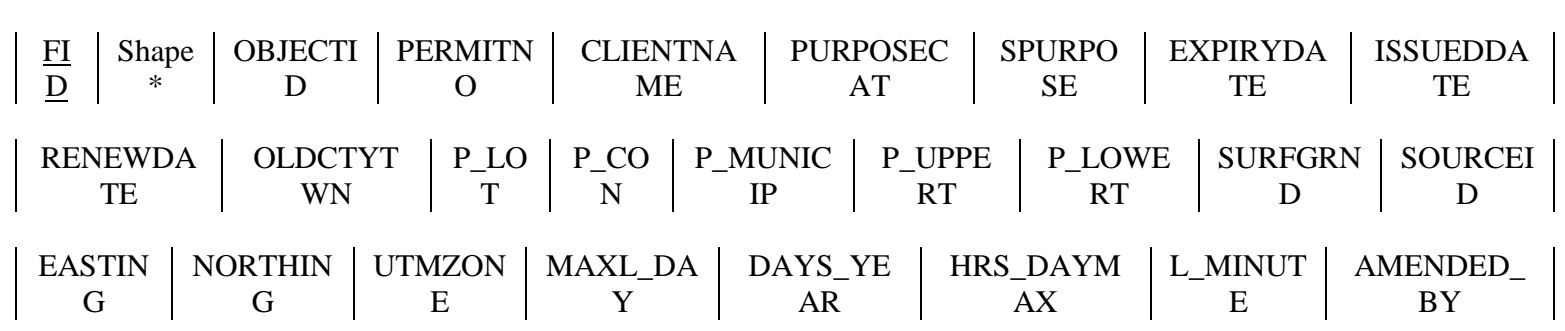

| EXPIRED_BY | PERMIT_END | ACTIVE | LATITUDE | LONGITUDE | LINK_TO_SW | SOURC_CITY |

| ISSUE_TICK | EXPIRY_TIC |

UTPrimaryWaterSupply_2014.shp

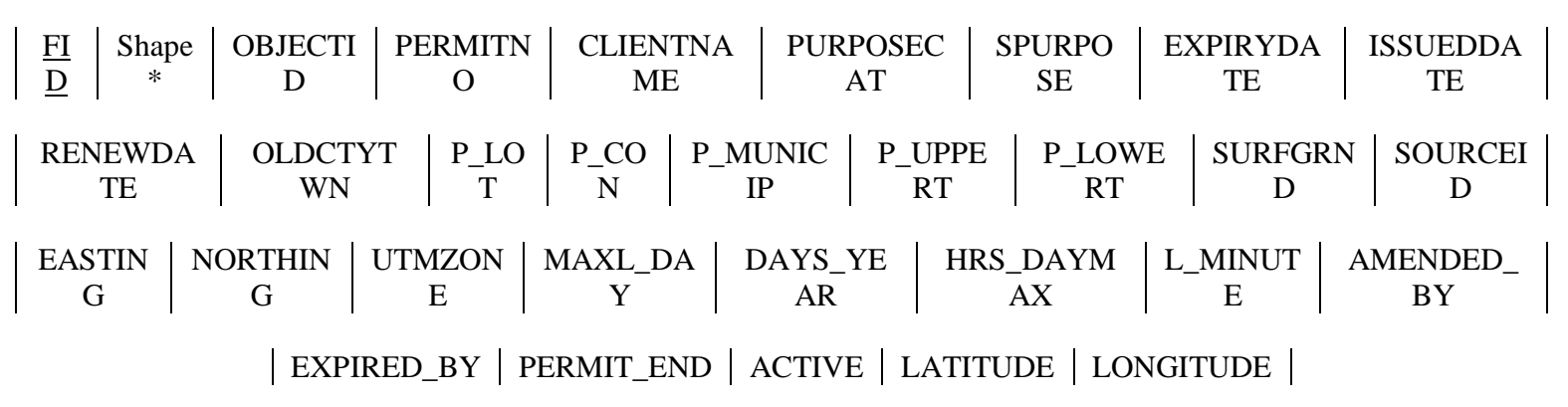




\section{Appendix B}

\section{Creating the hydrologic extension in Netlogo}

Netlogo modeling environment allows users to write external procedures in Java or other object-oriented programming languages, and then use them to support the agent-based models. This Netlogo feature is fully utilized to create an external hydrologic extension based on the Java code provided by Prodanovic(2007). To create a fully operational extension, Netlogo requires a folder with the following files:

A JAR File with the exact same name as the extension. JAR file must contain:

- At least one or more classes that implement org.nlogo.api.Primitive;

- A main class that implements org.nlogo.api.ClassManager;

And a Netlogo Manifest file that contains following tags:

- Manifest-Version (always 1.0)

- Extension-Name, defines the extension name

- Class-Manager, the full name of a class implementing org.nlogo.api.ClassManager.

Also, created extension must include netlogo.jar in the class path.

\section{Details on the Netlogo's Hydrology Extension}

\section{Extension folder}

The first step is to create a folder that will contain all necessary items. The name of the folder is the name of the extension, in our case: hydroexample.

\section{Extension primitives}

The new primitives are created as one or more Java classes. Netlogo contains two types of primitives: commands and reporters. Commands execute an action, while reporters return values. In order to create a new primitive, we need to create a class that implements the interface org.nlogo.api.Command or org.nlogo.api.Reporter to extend existing org.nlogo.api.Primitive. In our case, we write an extension that provides a single reporter named list-hydrology. 
DefaultReporter requires that we implement:

Object report (Argument args[], Context context)

throws ExtensionException;

Since our reporter takes an argument, we also implement:

Syntax getSyntax();

Here's the implementation of our reporter, in a file called src/ListHydrology.java:

import org.nlogo.api. *;

import java.io.*;

import java.awt.*;

import java.awt.event. *;

import javax.swing. *;

public class ListHydrology extends DefaultReporter

l

// take one number as input, report a list

public Syntax getSyntax()

f

return Syntax.reporterSyntax(

new int [] \{Syntax.NumberType(),Syntax.NumberType(), Syntax.NumberType(),

Syntax.NumberType(), Syntax.NumberType(), Syntax.NumberType(),

Syntax.NumberType(),

Syntax.NumberType()\}, Syntax.WildcardType());

\} 


\section{public Object report(Argument args[], Context context) throws ExtensionException \{}

// to create a NetLogo list for the result

LogoListBuilder list = new LogoListBuilder();

// Reads arguments communicated from the Netlogo model int Scnumb;

try

\{

Scnumb $=\operatorname{args}[0]$. getIntValue( $) ;$

\}

$\operatorname{catch}($ LogoException e $)$

\{

throw new ExtensionException( e.getMessage()) ;

\}

if $($ Scnumb $<0)$

\{

// signals a NetLogo runtime error to the modeler throw new ExtensionException "Input must be 1, 2, or 3");

\}

int $n$; 


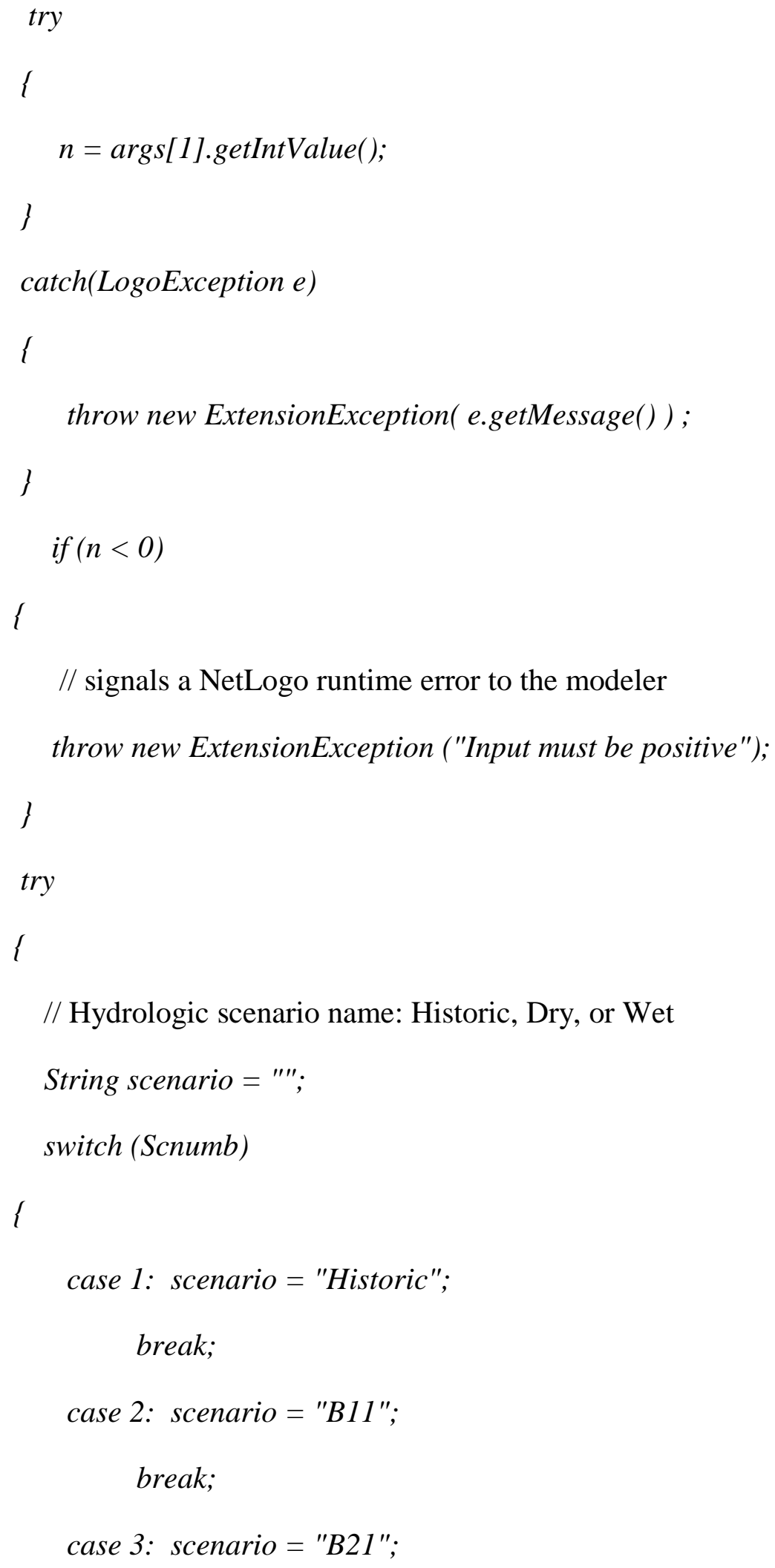


break;

\}

// get the current directory, location of the main file

String curDir = "C:/Program Files/NetLogo 5.0.5";

// this is the data for historically identical WG Scenario

String inputDir = curDir + "//Data//Daily//WGScenarios//" + scenario + "//";

String outputDir $=$ curDir + "//Data//Daily//WGScenarios//" + scenario + "//IntermediateFiles//";

double[] FPLMiddlesex = new double[240];

double[] FPLOxford = new double[240];

double[] FPLPerth = new double[240];

double[] FVMiddlesex = new double[240];

double[] FVOxford = new double[240];

double[] FVPerth = new double[240];

String outputDataDir = curDir + "//Data//";

if $(n==1)$

l

DataWriter FPLMiddlesexOut = new DataWriter(outputDataDir + "FPLMiddlesex.txt"); 
DataWriter FPLOxfordOut = new DataWriter(outputDataDir + "FPLOxford.txt");

DataWriter FPLPerthOut = new DataWriter(outputDataDir + "FPLPerth.txt");

DataWriter FVMiddlesexOut = new DataWriter $($ outputDataDir + "FVMiddlesex.txt");

DataWriter FVOxfordOut = new DataWriter(outputDataDir + "FVOxford.txt");

DataWriter FVPerthOut = new DataWriter(outputDataDir + "FVPerth.txt");

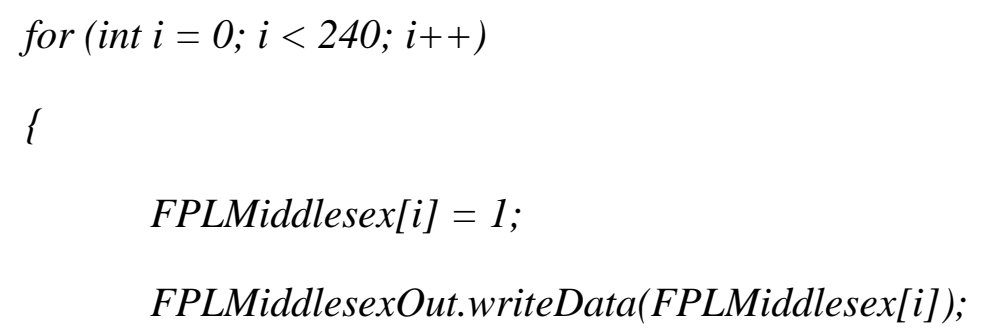

FPLOxford $[i]=1$

FPLOxfordOut.writeData(FPLOxford[i]);

FPLPerth $[i]=1$;

FPLPerthOut.writeData(FPLPerth[i]);

FVMiddlesex $[i]=1$;

FVMiddlesexOut.writeData(FVMiddlesex[i]);

FVOxford $[i]=1$

FVOxfordOut.writeData(FVOxford[i]);

FVPerth $[i]=1$; 
FVPerthOut.writeData(FVPerth[i]);

\}

FPLMiddlesexOut.closeFile();

FPLOxfordOut.closeFile();

FPLPerthOut.closeFile();

FVMiddlesexOut.closeFile();

FVOxfordOut.closeFile();

FVPerthOut.closeFile();

\}

DataReader FPLMiddlesexIn = new DataReader(outputDataDir + "FPLMiddlesex.txt");

DataReader FPLOxfordIn = new DataReader(outputDataDir + "FPLOxford.txt");

DataReader FPLPerthIn = new DataReader(outputDataDir + "FPLPerth.txt");

DataReader FVMiddlesexIn = new DataReader(outputDataDir + "FVMiddlesex.txt");

DataReader FVOxfordIn = new DataReader(outputDataDir + "FVOxford.txt");

DataReader FVPerthIn = new DataReader(outputDataDir + "FVPerth.txt");

for (int $i=0 ; i<240 ; i++)$

\{

FPLMiddlesex[i] = FPLMiddlesexIn.readCurrentData();

FPLOxford $[i]=$ FPLOxfordIn.readCurrentData () ;

FPLPerth $[i]=$ FPLPerthIn.readCurrentData(); 


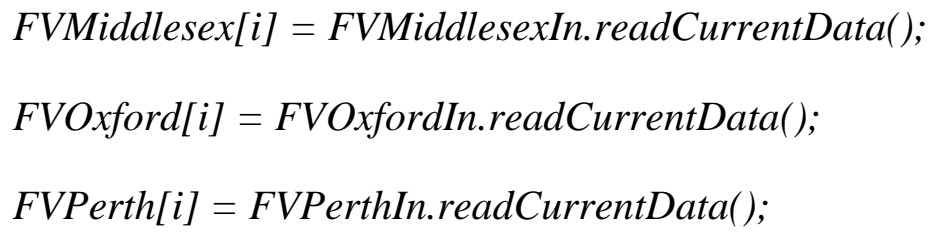


double FractionPavedLandOxford;

// use typesafe helper method from

// org.nlogo.api.Argument to access argument

try \{

FractionPavedLandOxford $=$ args[3].getDoubleValue();

\}

catch(LogoException e) \{

throw new ExtensionException( e.getMessage()));

\}

if (FractionPavedLandOxford $<0)$ \{

// signals a NetLogo runtime error to the modeler

throw new ExtensionException

("FractionPavedLandOxford must be positive");

\}

FPLOxford $[n-1]=$ FractionPavedLandOxford;

double FractionPavedLandPerth;

// use typesafe helper method from

// org.nlogo.api.Argument to access argument 
$\operatorname{try}\{$

FractionPavedLandPerth $=$ args [4].getDoubleValue( $)$;

\}

catch(LogoException e) \{

throw new ExtensionException( e.getMessage()) ;

\}

if (FractionPavedLandPerth $<0)\{$

// signals a NetLogo runtime error to the modeler

throw new ExtensionException

("FractionPavedLandPerth must be positive");

\}

FPLPerth[n-1] = FractionPavedLandPerth;

DataWriter FPLMiddlesexOut = new DataWriter(outputDataDir + "FPLMiddlesex.txt");

DataWriter FPLOxfordOut = new DataWriter(outputDataDir + "FPLOxford.txt");

DataWriter FPLPerthOut = new DataWriter(outputDataDir + "FPLPerth.txt");

for (int $i=0 ; i<240 ; i++)$

\{

FPLMiddlesexOut.writeData(FPLMiddlesex[i]);

FPLOxfordOut.writeData(FPLOxford[i]); 
FPLPerthOut.writeData(FPLPerth[i]);

\}

FPLMiddlesexOut.closeFile();

FPLOxfordOut.closeFile();

FPLPerthOut.closeFile();

double FractionVegetationMiddlesex;

// use typesafe helper method from

// org.nlogo.api.Argument to access argument

try \{

FractionVegetationMiddlesex $=\operatorname{args}[5]$. getDoubleValue();

\}

catch (LogoException e) \{

throw new ExtensionException( e.getMessage()) ;

\}

if (FractionVegetationMiddlesex $<0)$ \{

// signals a NetLogo runtime error to the modeler

throw new ExtensionException

("FractionVegetationMiddlesex must be positive");

\} 
FVMiddlesex $[n-1]=$ FractionVegetationMiddlesex;

double FractionVegetationOxford;

// use typesafe helper method from

// org.nlogo.api.Argument to access argument

$\operatorname{try}\{$

FractionVegetationOxford $=\operatorname{args[6].getDoubleValue();}$

\}

catch(LogoException e) \{

throw new ExtensionException( e.getMessage());

\}

if (FractionVegetationOxford $<0)\{$

// signals a NetLogo runtime error to the modeler

throw new ExtensionException

("FractionVegetationOxford must be positive");

\}

FVOxford $[n-1]=$ FractionVegetationOxford ;

double FractionVegetationPerth; 
// use typesafe helper method from

// org.nlogo.api.Argument to access argument

$\operatorname{try}\{$

FractionVegetationPerth $=$ args[7].getDoubleValue () ;

\}

catch(LogoException e) \{

throw new ExtensionException( e.getMessage());

\}

if $($ FractionVegetationPerth $<0)\{$

// signals a NetLogo runtime error to the modeler

throw new ExtensionException

("FractionVegetationPerth must be positive");

\}

FVPerth $[n-1]=$ FractionVegetationPerth;

DataWriter FVMiddlesexOut = new DataWriter(outputDataDir + "FVMiddlesex.txt");

DataWriter FVOxfordOut = new DataWriter(outputDataDir + "FVOxford.txt");

DataWriter FVPerthOut = new DataWriter(outputDataDir + "FVPerth.txt");

for (int $i=0 ; i<240 ; i++)$

\{

FVMiddlesexOut.writeData(FVMiddlesex[i]); 
FVOxfordOut.writeData(FVOxford[i]);

FVPerthOut.writeData(FVPerth[i]);

\}

FVMiddlesexOut.closeFile();

FVOxfordOut.closeFile();

FVPerthOut.closeFile();

// populate the result list, NetLogo numbers are always Doubles

$/ /$ try \{

if $(($ currentDate.getDay ()$==$ currentDate.getDaysInMonth ()$) \& \&$ (currentDate.getHour ()$>=19))\{$

MyMonth = MyMonth + 1;

// obtains monthly averages for the hydrologic output $/ /\{\{\{$ // computes the number of user times steps this month userTimeStepsInMonth $=$ currentDate.getDaysInMonth () * (24 / userTimeStep); 
// this is average monthly GWRecharge, in [m3/yr]

GWRechargeMiddlesex $=$ GWRechargeMiddlesex $/$ userTimeStepsInMonth;

GWRechargeOxford = GWRechargeOxford / userTimeStepsInMonth;

GWRechargePerth $=$ GWRechargePerth $/$ userTimeStepsInMonth;

// this is average monthly flow, in [cms]

jnByronSG = jnByronSG / userTimeStepsInMonth;

jnIngersollSG = jnIngersollSG / userTimeStepsInMonth;

jnStMarysSG = jnStMarysSG / userTimeStepsInMonth;

// Adds values to a list to be returned to Netlogo

if $($ MyMonth $==n)$ \{

list.add(Double.valueOf(MyMonth));

list.add(GWRechargeMiddlesex);

list.add(GWRechargeOxford);

list.add(GWRechargePerth);

list.add(jnByronSG);

list.add(jnIngersollSG);

list.add(jnStMarysSG); 


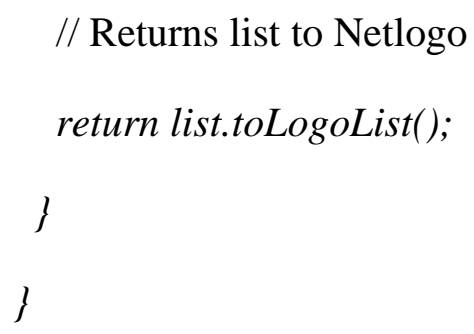

\section{Create a Class Manager}

Each extension must contain a class that includes the interface org.nlogo.api.ClassManager. This Class Manager defines the primitives used in the extension. Here is the Class Manager for our Hydrology extension:

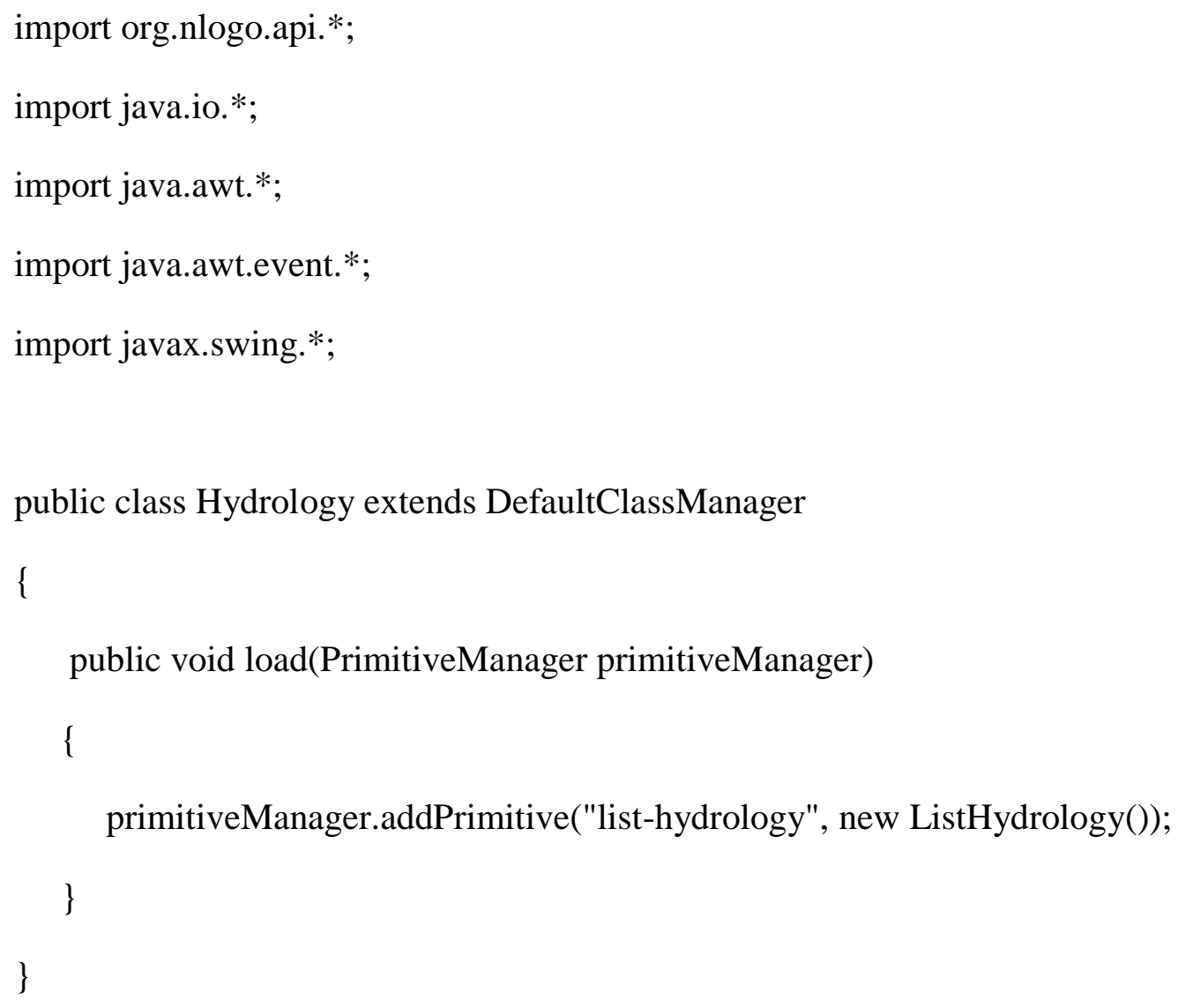




\section{A Manifest file}

Every extension must contain a manifest text file which communicates to Netlogo the name of the extension and the location of the Class Manager. The manifest file contains three tags:

- Extension name

- Class Manager

- Netlogo Extension API Version

Here is the manifest.txt file for the hydrology extension:

Manifest-Version: 1.0

Netlogo-Extension-API-Version: 5.0

Class-Manager: Hydrology

Extension-Name: hydroexample

\section{JAR File}

Final step is to create an extension's JAR file. First we need to compile the classes, and then create a JAR that contains the resulting class files and the manifest. In Hydrology extension example:

jar -cvfm hydroexample.jar c:VHYDROLOGYmanifest.txt -C c: $\mathrm{HYDROLOGY*}$ 


\section{Appendix C}

\section{Netlogo Variables}

This appendix lists all variables for each individual entity defined by the model. Also, the list includes global and patch model variables.

\begin{tabular}{|c|c|}
\hline \multicolumn{2}{|r|}{ Global variables (globals) } \\
\hline elevation-dataset & Utility variable for DEM dataset (Raster dataset) \\
\hline rivers-dataset & Utility variable for River dataset (Shape dataset) \\
\hline landuse-dataset & Utility variable for Land use dataset (Raster dataset) \\
\hline subwatersheds-dataset & Utility variable for sub-basin dataset (Raster dataset) \\
\hline municipalities-dataset & Utility variable for municipality dataset (Raster dataset) \\
\hline municipalities-shape-dataset & Utility variable for municipality dataset (Shape dataset) \\
\hline ut-sb-shape-dataset & Utility variable for UT sub-basins dataset (Shape dataset) \\
\hline cities-dataset & Utility variable for UT cities dataset (Shape dataset) \\
\hline flow-gauging-stations-dataset & $\begin{array}{l}\text { Utility variable for UT gauging stations dataset (Shape } \\
\text { dataset) }\end{array}$ \\
\hline industrial-waterusers-dataset & $\begin{array}{l}\text { Utility variable for Industrial PTTW dataset (Shape } \\
\text { dataset) }\end{array}$ \\
\hline agricultural-waterusers-dataset & $\begin{array}{l}\text { Utility variable for Agricultural PTTW dataset (Shape } \\
\text { dataset) }\end{array}$ \\
\hline watersupply-waterusers-dataset & $\begin{array}{l}\text { Utility variable for Water Supply PTTW dataset (Shape } \\
\text { dataset) }\end{array}$ \\
\hline commercial-waterusers-dataset & $\begin{array}{l}\text { Utility variable for Commercial PTTW dataset (Shape } \\
\text { dataset) }\end{array}$ \\
\hline primary-system-dataset & Utility variable for Primary WSS dataset (Shape dataset) \\
\hline land-patches & Patches within UTRB \\
\hline agricultural-patches & Agricultural patches within UTRB \\
\hline london-patches & Patches occupied by the City of London \\
\hline forestry-patches & Groups Forestry patches in the basin \\
\hline
\end{tabular}




urban-patches
border
simulation-counter
seasonal-list-item-counter
city-of-london
total-water
month
fraction-vegetated-land-middlesex-
output
fraction-vegetated-land-perth-
output
groundwater-recharge-1
fraction-paved-land-perth-output
groundwater-recharge-2
groundwation-hater-recharge-3
scenario-number
surface-water-1
surface-water-2

urban-patches

Urban patches in UTRB

UTRB border patches

Main simulation time counter

Seasonal variation list counter

Patches occupied by London

Hydrologic Extension: A list of variables imported from the hydrologic model

Hydrologic Extension: Current time step

Hydrologic Extension: Groundwater recharge per municipality 1

Hydrologic Extension: Groundwater recharge per municipality 2

Hydrologic Extension: Groundwater recharge per municipality 3

Hydrologic Extension: Runoff at gauging station 1

Hydrologic Extension: Runoff at gauging station 2

Hydrologic Extension: Runoff at gauging station 3

Hydrologic Extension: Scenario (Wet, Dry, Historic)

Hydrologic Extension: Percentage of paved land in Middlesex county

Hydrologic Extension: Percentage of paved land in Oxford county

Hydrologic Extension : Percentage of paved land in Perth county

Hydrologic Extension : Percentage of vegetated land in Middlesex county

Hydrologic Extension: Percentage of vegetated land in Oxford county

Hydrologic Extension: Percentage of vegetated land in Perth county

Hectares Under Corn 


$\begin{array}{cl}\text { corn-yield-revenue } & \text { Corn Economic Revenue } \\ \text { hay-fields-hectares } & \text { Hectares Under Hay } \\ \text { hay-yield-revenue } & \text { Hay Economic Revenue } \\ \text { barley-fields-hectares } & \text { Hectares Under Barley } \\ \text { barley-yield-revenue } & \text { Barley Economic Revenue } \\ \text { mixed-fields-hectares } & \text { Hectares Mixed System } \\ \text { mixed-yield-revenue } & \text { Mixed System Economic Revenue }\end{array}$




\begin{tabular}{cl}
\hline elevation & Patches Variables (patches-own) \\
is-land-patch & Defines elevation for each cell \\
is-border & Patches within the Upper Thames River Basin \\
is-ag & Defines border patches \\
is-london & Patches dedicated to agriculture \\
is-urban & Patches occupied by the City of London \\
landuse-category & Urbanized patches in the UTRB, excluding the City of \\
subwatershed-ID & London \\
municipalities-ID & Defines the land use category \\
crop-yield-tonnes-per-hectare & Defines the sub-basin for each cell \\
dollars-per-tonne & Defines the municipality for each cell \\
\hline
\end{tabular}




\begin{tabular}{|c|c|}
\hline \multicolumn{2}{|c|}{ Agricultural Users (agricultural-pttws-own) } \\
\hline sb-id & $\begin{array}{l}\text { Creates a link between an Industrial Water User and } \\
\text { Responsible Manager }\end{array}$ \\
\hline ag-user-id & Unique agricultural user ID \\
\hline ag-specific-purpose & $\begin{array}{l}\text { Agricultural Specific purpose }-7 \text {. Types ( Field and } \\
\text { Pasture Crops, Fruit Orchards, Market Gardens/Flowers, } \\
\text { Nursery, SOD Farm, Tobacco, Other Agricultural) }\end{array}$ \\
\hline ag-issue-date & The date when the license is issued [Date Format] \\
\hline ag-expiry-date & The date when the license expires [Date Format] \\
\hline ag-issue-ticks & The date when the license is issued [Netlogo Format] \\
\hline ag-expiry-ticks & The date when the license expires [Netlogo Format] \\
\hline ag-max-litres-per-day & Maximum allowed liters per day to be taken [liters/day] \\
\hline ag-days-per-year & $\begin{array}{l}\text { Maximum number of days per year allowed for taking } \\
\text { [days] }\end{array}$ \\
\hline ag-total-annual-demand & Total demand per year $\left[\mathrm{m}^{3} /\right.$ year $]$ \\
\hline ag-monthly-demand & Actual monthly demand $\left[\mathrm{m}^{3} /\right.$ month] \\
\hline agricultural-seasonal-variation-list & Seasonal agricultural demand variation list [monthly] \\
\hline ag-water-footprint & $\begin{array}{l}\text { Water used }\left[\mathrm{m}^{3}\right] \text { for production of } 1 \mathrm{~kg} \text { of agricultural } \\
\text { commodity }\left[\mathrm{m}^{3} / \mathrm{kg}\right]\end{array}$ \\
\hline ag-produced-commodities & $\begin{array}{l}\text { Produced commodities in }[\mathrm{kg}] \text { based on the water demand } \\
\text { and agricultural water footprint }\end{array}$ \\
\hline ag-gross-economic-revenue & $\begin{array}{l}\text { Gross economic revenue based on produced goods }[\mathrm{kg}] \\
\text { and unit price }[\$ / \mathrm{kg}]\end{array}$ \\
\hline ag-unit-commodity-price & Price per unit $\$ / \mathrm{kg}$ of produced agricultural commodity \\
\hline ag-water-use-costs & $\begin{array}{l}\text { Economic Costs of Water Utilization for Agriculture based } \\
\text { on the Agricultural Water Price }\end{array}$ \\
\hline active? & Defines if an agent is active in current time step \\
\hline
\end{tabular}




\begin{tabular}{cl}
\hline \multicolumn{2}{c}{ Commercial Users (commercial-pttws-own) } \\
\hline co-user-id & $\begin{array}{l}\text { Creates a link between an Industrial Water User and } \\
\text { Responsible Manager }\end{array}$ \\
co-specific-purpose & Unique Commercial user ID \\
co-issue-date & 2 Types of commercial users \\
co-expiry-date & The date when the license is issued (Date Format) \\
co-issue-ticks & The date when the license expires (Date Format) \\
co-expiry-ticks & The date when the license is issued (Netlogo Format) \\
co-max-litres-per-day & The date when the license expires (Netlogo Format) \\
co-days-per-year & Maximum allowed liters per day to be taken \\
co-total-annual-demand & Maximum number of days per year allowed for taking \\
co-monthly-demand & Total demand per year \\
active? & Actual monthly demand \\
\hline commercial-seasonal-variation-list & Seasonal agricultural demand variation list (monthly) \\
& Defines if an agent is active in current time step \\
\hline
\end{tabular}




\begin{tabular}{cl}
\hline \multicolumn{1}{c}{ Water Supply Users (water-supply-pttws-own) } \\
source-for-city & $\begin{array}{l}\text { Creates a link between an Industrial Water User and } \\
\text { Responsible Manager }\end{array}$ \\
ws-issue-date & Unique Water Supply user ID \\
ws-expiry-date & The date when the license is issued (Date Format) \\
ws-issue-ticks & The date when the license expires (Date Format) \\
ws-expiry-ticks & The date when the license is issued (Netlogo Format) \\
ws-max-litres-per-day & The date when the license expires (Netlogo Format) \\
ws-days-per-year & Maximum allowed liters per day to be taken \\
ws-max-annual-capacity & Maximum number of days per year allowed for taking \\
ws-max-monthly-capacity & Total demand per year \\
water-supply-seasonal-variation-list & Seasonal (monthly) variation list \\
active? & Defines if an agent is active in current time step \\
\hline
\end{tabular}




\begin{tabular}{cl}
\hline \multicolumn{2}{c}{ Primary Water Supply Systems (primary-wsss-own) } \\
\hline pwss-name & Unique name \\
pwss-max-litres-per-day & $\begin{array}{l}\text { Maximum allowed liters per day to be taken (Designed } \\
\text { capacity) }\end{array}$ \\
pwss-total-annual-capacity & Maximum number of days per year allowed for taking \\
ws-max-monthly-capacity & Actual monthly capacity \\
primary-water-system-seasonal- & Seasonal (monthly) variation list \\
variation-list &
\end{tabular}




\begin{tabular}{cl}
\hline \multicolumn{2}{c}{ Managers on Sub-basin Level (subbasin-managers-own) } \\
\hline municipality-id & Unique ID identifier \\
percentage-of-municipality-area & Pinks a subbasin manager to responsible municipality \\
total-ag-monthly-demand & $\begin{array}{l}\text { Total Monthly Agricultural Demand Claimed From the } \\
\text { Sub-basin Managers }\end{array}$ \\
$\begin{array}{c}\text { subbasin-annual-nonpermitted- } \\
\text { domestic-use }\end{array}$ & Non-permitted annual water use (Domestic) \\
subbasin-annual-nonpermitted- & Non-permitted annual water use (Agriculture) \\
agriculture-use & \\
subbasin-monthly-nonpermitted- & Non-permitted monthly water use (Domestic) \\
domestic-use & \\
subbasin-monthly-nonpermitted- & Non-permitted monthly water use (Agriculture) \\
agriculture-use &
\end{tabular}




\begin{tabular}{cl}
\hline \multicolumn{2}{c}{ Managers on County Level (municipal-managers-own) } \\
\hline municipality-id & Unique identified \\
available-groundwater & Municipality name \\
municipal-total-water-demand & Troundwater recharge per municipality \\
municipal-current-water-balance & Water balance per municipality \\
\hline
\end{tabular}




\section{Appendix D}

\section{IHSEM-Model installation}

Package contains three files:

1. IHSEM-UTRB.rar (Netlogo model and databases)

2. hydroexample.rar (Hydrologic extension)

3. Data.rar (Precipitation and temperature inputs data)

Installation process is done in 6 steps.

Step 1:

This model requires Java JRE 1.5 or later versions installed on the machine.

The IHSEM-UTRB model is developed in Netlogo 5.0.5 modeling environment which can be downloaded from the Netlogo's official website:

https://ccl.northwestern.edu/netlogo/download.shtml

Install Netlogo to:

C: Program FilesWetLogo 5.0.5

Step 2:

After Netlogo installation, extract IHSEM-UTRB.rar, and copy file

IHSEM-UTRB.nlogo and data folder to:

C: Program FilesWetLogo 5.0.5 Jmodels

data folder contains all spatial databases. 
Step 3:

To install the hydrologic extension, create a folder hydroexample:

C: Program FilesWetLogo 5.0.5 \extensions Vydroexample

And copy the file hydroexample.jar from hydrologicextension.rar into this folder.

Step 4:

Extracted Data folder from Data.rar (that contains precipitation and temperature inputs for the hydrologic model) copy to:

C:Program FilesWetLogo 5.0.5

Step 5:

Before initializing the model, it is necessary to install the Time extension. Details on installation are presented here:

https://github.com/colinsheppard/time/\#installation

Step 6:

Open IHSEM-UTRB.nlogo, select the parameters, initialize and run the simulation. 


\section{Curriculum Vitae}

Name:

Post-secondary Education and Degrees:

Honours and Awards:

Related Work
Experience
Vladimir NIKOLIC

Faculty of Civil Engineering and Architecture, University of Nis Nis, Serbia 1999-2007 MSc

École d'Ingénieurs Polytechnique de l'Université Nice - Sophia Antipolis, Nice, France 2007-2009 MSc

Department of Civil and Environmental Engineering, The University of Western Ontario

London, Ontario, Canada

2011-2015 PhD

Erasmus Mundus Scholarship, European Union 2007-2009

Teaching and Research Assistant The University of Western Ontario 2011-2014

\section{Publications:}

Nikolic V., Simonovic S.P. and Milicevic D. (2013). "Analytical support for integrated water resources management: a new method for addressing spatial and temporal variability." Water Resources Management Journal, January 2013, Volume 27, Issue 2, pp 401-417

Nikolic V., Simonovic S.P. and Milicevic D. (2013). "Generic Modeling Framework for Integrated Water Resources Management." Proceedings 21st Canadian Hydrotechnical Conference, Banff, Alberta, Canada, May 14-17, 2013. 